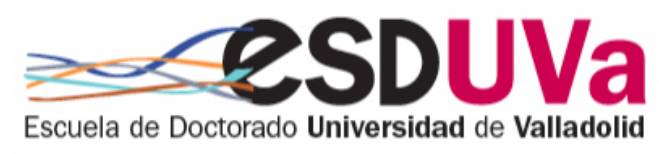

Universidad deValladolid

PROGRAMA DE DOCTORADO EN INGENIERÍA

INDUSTRIAL

TESIS DOCTORAL:

\title{
METODOLOGÍAS PARA LA ESTIMACIÓN DE LOS PARÁMETROS MODALES EN PASARELAS PEATONALES: APLICACIÓN A LA PASARELA DEL MUSEO DE LA CIENCIA DE VALLADOLID
}

Presentada por $\mathrm{D}^{\mathrm{a}}$ Noelia Frechilla Alonso para optar al grado de Doctora por la Universidad de Valladolid

Dirigida por:

Dr. Mariano Cacho Pérez. 

ESCUELA DE INGENIERÍAS INDUSTRIALES

DEPARTAMENTO DE CONSTRUCCIONES ARQUITECTÓNICAS, INGENIERÍA DEL TERRENO Y MECÁNICA DE LOS MEDIOS CONTINUOS Y TEORÍA DE ESTRUCTURAS

\section{TESIS DOCTORAL:}

\section{METODOLOGÍAS PARA LA ESTIMACIÓN DE LOS} PARÁMETROS MODALES EN PASARELAS PEATONALES: APLICACIÓN A LA PASARELA DEL MUSEO DE LA CIENCIA DE VALLADOLID

Presentada por NOELIA FRECHILLA ALONSO, Ingeniera de Caminos,

Canales y Puertos por la Escuela Técnica Superior de Ingenieros de Caminos, Canales y Puertos de la Universidad de A Coruña.

Dirigida por MARIANO CACHO PÉREZ, Profesor del Departamento de Construcciones Arquitectónicas, Ingeniería del Terreno y Mecánica de los Medios Continuos y Teoría de Estructuras, Universidad de Valladolid

Esta Tesis Doctoral fue leída en la Escuela de Ingenierías Industriales de la Universidad de Valladolid, el día , estando compuesto el tribunal calificador por:

Presidente

Vocal primero
Vocal segundo

Secretario

Obteniendo la calificación de

Valladolid, de

de 2017 

El movimiento se demuestra andando

Diógenes de Sinope 

A mi madre, el mejor ejemplo de amor y de lucha que la vida me ha podido dar 



\section{Agradecimientos}

Quiero expresar mi más sincero agradecimiento a las personas e instituciones que han hecho posible la realización de esta Tesis Doctoral:

A mi director de Tesis, Dr. Mariano Cacho Pérez, por su orientación científica, colaboración y apoyo durante su realización.

A la Fundación Cartif y al Dr. Antolín Lorenzana Ibán por su cooperación y contribución en el desarrollo de la misma y a Javier Castaño y Norberto Ibán por su disposición y ayuda.

A mi familia por su ánimo y sus palabras que consiguieron que continuara y finalizara esta Tesis.

Muchas gracias por vuestras aportaciones. 



\section{Resumen}

Obras de ingeniería civil como las pasarelas peatonales, son estructuras cada vez más esbeltas, ambiciosas y complejas en las que los efectos dinámicos producidos por las solicitaciones a las que están sometidas pueden inducir, además de problemas de servicio, amplificaciones importantes y repetitivas de los esfuerzos y deformaciones que pueden afectar a la seguridad y viga a fatiga de la estructura.

Aunque las normativas recogen diversos criterios de evaluación de estos fenómenos, también reflejan la dificultad de evaluar con precisión el comportamiento dinámico de las estructuras, exigiendo en algunos casos un estudio dinámico riguroso.

El principal objetivo de este trabajo es validar diferentes métodos y herramientas de análisis modal y caracterización dinámica, aplicándolos a un caso real, una pasarela peatonal actualmente en servicio, y complementando un análisis dinámico anterior realizado sobre la misma. De esta forma se amplía el abanico de metodologías que faciliten la implantación del estudio de las vibraciones en las estructuras de obra civil, permitiendo que este tipo de acciones dinámicas no limiten la progresiva evolución que se está desarrollando en el diseño de estructuras cada vez más esbeltas.

El presente estudio se estructura como se describe a continuación:

- Estudios sobre la Pasarela del Museo de la Ciencia de Valladolid:

- Modelización de la estructura completa mediante el Software de Elementos Finitos ANSYS.

- Ajuste del modelo (FEM Updating) mediante la comparación de las frecuencias y formas modales logradas, con los resultados experimentales obtenidos en un estudio anterior. 
- Análisis modal y dinámico de la Pasarela del Museo de la Ciencia de Valladolid:

- Validación de una metodología para estimar los parámetros modales del sistema dinámico, partiendo de una representación en el espacio de estados mediante la técnica de identificación en el subespacio estocástico (SSI Stochastic Subspace Identificarion), y obteniendo mediante la adecuada matriz de transformación los parámetros físicos del sistema (matrices de masa, amortiguamiento y rigidez) que permiten calcular las masas modales y/o modos normalizados respecto a la matriz de masa.

- Validación de una segunda metodología en la que partiendo de nuevo de una representación en el espacio de estados mediante la técnica SSI, se escalan los modos de vibración a partir de la estimación de los residuos de la descomposición en fracciones parciales de la función en frecuencia (FRF Frequency Response Function) de las mediciones.

La aplicación práctica de las herramientas que se evalúan en este trabajo no se limita exclusivamente a las etapas de puesta en servicio y mantenimiento, ya que se incluye uno de los instrumentos, el análisis de elementos finitos (FEA - Finite Element Analysis), que con mayor profusión se está aplicando también en las fases más tempranas de los proyectos. El objetivo es que la implementación de este recurso en la etapa de diseño de este tipo de estructuras permita efectuar modificaciones o correcciones al menor coste tanto económico como en la calidad del proyecto, siendo necesario para su difusión el análisis de su viabilidad en casos actualmente en servicio.

Algunos de los puntos anteriores han supuesto aportaciones originales que han dado lugar a distintos artículos científicos publicados en revistas internacionales, recogidos éstos en la parte final del documento. 


\section{Abstract}

Civil engineering constructions like pedestrian footbridges are structures increasingly slender, ambitious and complex in which the dynamic effects caused by the loads to which they are subjected (pedestrians) may induce service problems and significant and repetitive amplifications of the efforts and deformations that may affect the safety and fatigue life of the structure.

Although some guidelines and regulations contain various evaluation criteria for these phenomena, they also reflect the difficulty of accurate assessment of the dynamic behavior of structures, requiring in some cases a rigorous dynamic study.

The main aim of this work is to validate different methods and tools of modal analysis and dynamic characterization, applying them to a real case, a pedestrian footbridge currently in service, and complementing a previous dynamic analysis performed on it. In this way, the range of methodologies that facilitate the implementation of the study of vibrations in civil structures is increased, allowing this type of dynamic actions not to limit the progressive evolution that is being developed in the design of this type of structures increasingly slender.

The present study is structured as follows:

- Studies on the Science Museum Footbridge of Valladolid:

○ Modeling of the complete structure using the software of finite element ANSYS.

- FEM Updating by comparing the calculated frequencies and modal modes, with the experimental ones obtained in an earlier study. 
- Modal and dynamic analysis of the Science Museum Footbridge of Valladolid:

- Validation of a methodology to estimate the modal parameters of the dynamic system, starting from a representation in the state space by SSI technique (Stochastic Subspace Identification), and obtaining, by the appropriate transformation matrix, the physical parameters of the system (mass, damping and stiffness matrices) which allows to calculate modal masses and / or normalized modes shapes with respect to the mass matrix.

- Validation of a second methodology in which starting again from a representation in the state space by SSI technique, vibration modes are scaled from the estimation of the residues of the decomposition in partial fractions of the Frequency Response Function (FRF) of the measurements.

The practical application of the tools evaluated in this work is not only limited to the service and maintenance stages, since it includes one of the instruments, the Finite Element Analysis (FEA), which has greater profusion at the earliest stages of the projects. The objective is that the implementation of this resource in the design stage of this type of structures allows modifications or corrections to be made at the lowest economic and quality cost of the project, being necessary for its diffusion the analysis of its feasibility in cases currently in service.

Some of the previous points have been original contributions that have given rise to different articles published in international journals, collected at the end of this document. 


\section{Índice general}

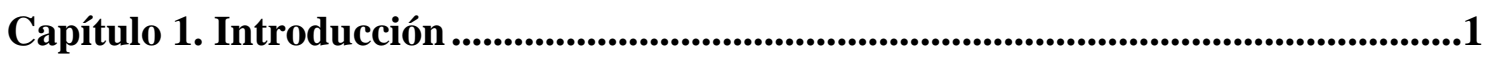

1.1. El tema en su contexto ........................................................... 1

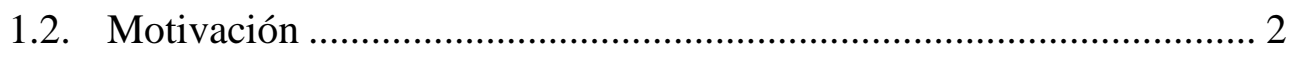

1.3. Antecedentes y estado actual ..................................................... 4

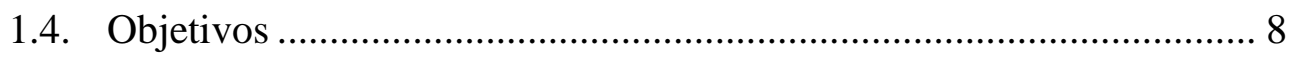

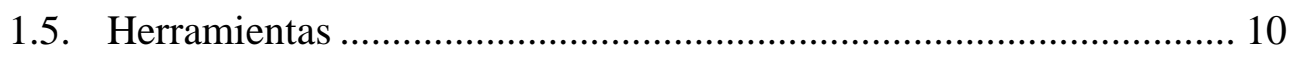

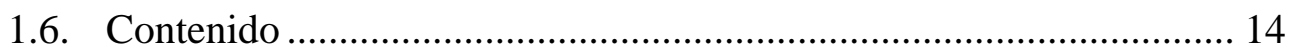

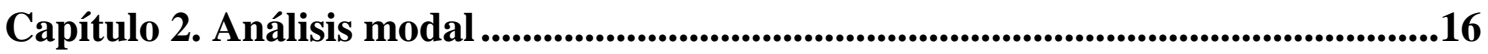

2.1. Teoría del análisis modal ............................................................ 20

2.1.1. Frecuencia natural ......................................................... 21

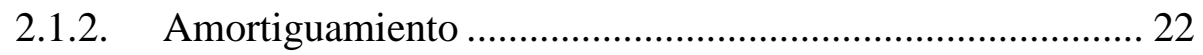

2.1.3. Resonancia ................................................................. 26

2.1.4. Función de respuesta en frecuencia .................................. 28

2.1.5. Modos de vibración........................................................... 31

2.2. Análisis modal experimental ....................................................... 35 


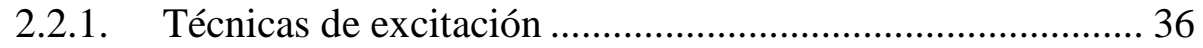

2.2.2. Medida de la excitación y la vibración ............................... 38

2.2.3. Análisis y procesado de las señales.................................... 39

2.3. Técnicas de análisis modal aplicadas a la Pasarela del Museo de la Ciencia. 43

Capítulo 3. Modelo mecánico ajustado. . .49

3.1. El método de elementos finitos ....................................................... 53

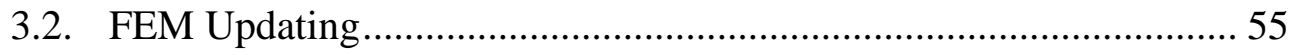

3.2.1. Métodos de optimización directa ...................................... 57

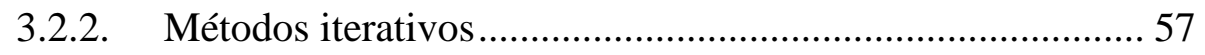

Capítulo 4. Métodos de escalado propuestos ...............................................................62

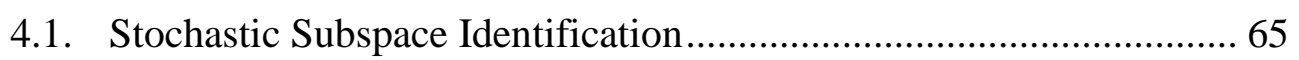

4.2. Problema simétrico de valores propios ........................................... 68

4.3. Escalado de los modos de vibración a partir de la función de respuesta en frecuencia.

5.1. Resultados de la modelización de la Pasarela del Museo de la Ciencia

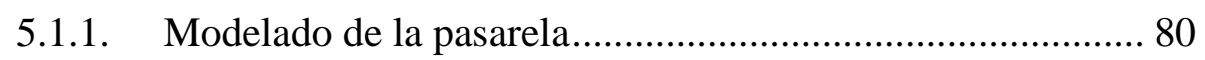

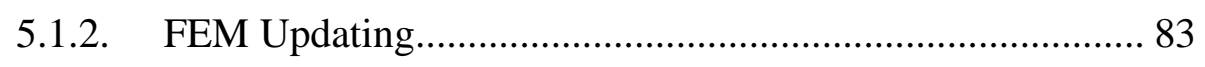

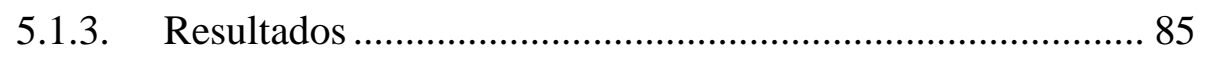

5.2. Resultados obtenidos aplicando las metodologías propuestas ........... 87

5.2.1. Problema simétrico de valores propios ............................... 87

5.2.2. Escalado de los modos de vibración a partir de la función de respuesta en frecuencia 
Capítulo 6. Conclusiones.............................................................................................99

6.1. Modelo de elementos finitos de la pasarela completa..................... 101

6.2. Métodos de escalado propuestos .................................................. 102

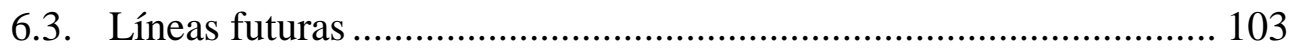

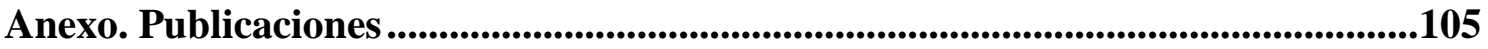

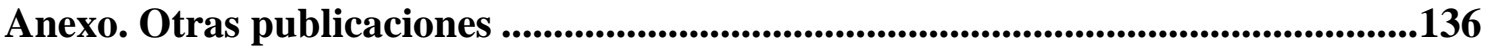

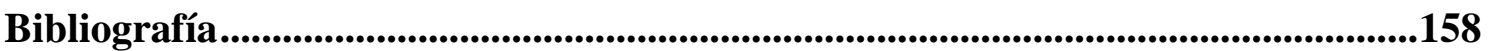





\section{Listado de abreviaturas}

ASCE American Society of Civil Engineers (Sociedad Americana de Ingenieros Civiles)

CFDD Curve-Fit Frequency Domain Decomposition (Descomposición en el Dominio de la Frecuencia con Ajuste de la Curva)

DFT Discrete Fourier Transform (Transformada Discreta de Fourier)

EFDD Enhanced Frequency Domain Decomposition (Descomposición en el Dominio de la Frecuencia Mejorada)

EMA Experimental Modal Analysis (Análisis Modal Experimental tradicional)

FDD Frequency Domain Decomposition (Descomposición en el Dominio de la Frecuencia)

FEA Finite Element Analysis (Análisis de Elementos Finitos)

FEM Finite Element Method (Método de Elementos Finitos)

FFT Fast Fourier Transform (Transformada Rápida de Fourier)

FRF Función Respuesta en Frecuencia

GDL Grados de Libertad

LVDT Linear Variable Differential Transformer (Transformador Diferencial de Variación Lineal)

MAC Modal Assurance Criterion (Criterio de Confianza Modal)

MSF $\quad$ Modal Scale Factor (Factor de Escala Modal)

OMA Operational Modal Analysis (Análisis Modal Operacional) 
OMAX Operacional Modal Analysis with eXogenous inputs (Análisis Modal Operacional con entradas eXógenas)

PMC Pasarela del Museo de la Ciencia

$\mathrm{rad} / \mathrm{s} \quad$ radianes por segundo

SSI Stochastic Subspace Identification (Identificación en el Subespacio Estocástico)

TMD Tuned Mass Dampers (Absorbedor pasivo de vibraciones) 


\section{Índice de figuras}

Figura 2-1. Oscilador armónico simple ..........................................................................20

Figura 2-2. Movimiento de un sistema crítico.................................................................24

Figura 2-3. Movimiento de un sistema subcrítico ..................................................................25

Figura 2-4. Amplificación dinámica en función de la razón de frecuencias para varios

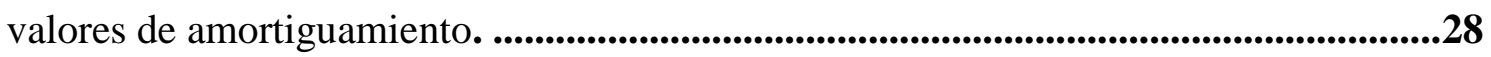

Figura 2-5. Pasarela del Museo de la Ciencia (Valladolid). Vista general........................43

Figura 2-6. Pasarela del Museo de la Ciencia (Valladolid). Vista del vano principal.....44

Figura 2-7. Pasarela del Museo de la Ciencia (Valladolid). Detalle del pretensado

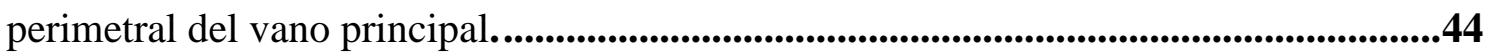

Figura 2-8. Pasarela del Museo de la Ciencia (Valladolid). Vista del vano 2..................45

Figura 2-9. Acelerómetro empleado en el OMA...................................................................46

Figura 2-10. Equipo de medida utilizado en el OMA. ..........................................................46

Figura 2-11. Excitador electrodinámico utilizado en el EMA.................................................47

Figura 5-1. Celosía tubular tridimensional de sección transversal hexagonal. .................80

Figura 5-2. Sección transversal del pretensado del vano 3.......................................................81

Figura 5-3. Estructura principal.............................................................................................................81

Figura 5-4. Estructura del tablero. ............................................................................81 
Figura 5-5. Apoyo central......................................................................................................82

Figura 5-6. Vista general del modelo completo de la pasarela en ANSYS......................83

Figura 5-7. Primeros cuatro modos verticales de vibración de la pasarela obtenidos experimentalmente. .84

Figura 5-8. Primer modo vertical: $\mathrm{f}_{1}=1.224 \mathrm{~Hz}$ obtenido con el modelo en ANSYS.

Figura 5-9. Segundo modo vertical: $\mathrm{f}_{2}=2.842 \mathrm{~Hz}$ obtenido con el modelo en ANSYS. . .85

Figura 5-10. Tercer modo vertical: $f_{3}=3.662 \mathrm{~Hz}$ obtenido con el modelo en ANSYS.

Figura 5-11. Cuarto modo vertical: $\mathrm{f}_{4}=4.914 \mathrm{~Hz}$ obtenido con el modelo en ANSYS.

Figura 5-12. Viga continua que se emplea como ejemplo de aplicación de la metodología descrita. .89 


\section{Índice de tablas}

Tabla 5-1. Valores de frecuencia de los primeros modos de flexión de la pasarela

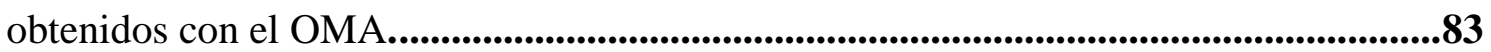

Tabla 5-2. Estimaciones de masa modal del tercer modo vertical. ..................................84

Tabla 5-3. Comparativa entre las frecuencias obtenidas experimentalmente y las obtenidas mediante la modelización de la pasarela en ANSYS. .86

Tabla 5-4. Comparativa entre los parámetros modales estimados mediante el problema simétrico de valores propios y los obtenidos analíticamente (viga biapoyada). .88 Tabla 5-5.- Comparativa entre los modos de vibración estimados mediante el problema simétrico de valores propios y los obtenidos analíticamente (viga biapoyada). .88

Tabla 5-6.- Comparativa entre los modos de vibración normalizados estimados mediante el problema simétrico de valores propios y los obtenidos analíticamente (viga biapoyada).

Tabla 5-7. Comparativa entre los parámetros modales estimados mediante el problema simétrico de valores propios y los obtenidos analíticamente (viga continua de tres vanos). 
Tabla 5-8.- Comparativa entre los modos de vibración estimados mediante el problema simétrico de valores propios y los obtenidos analíticamente (viga continua de tres vanos).

Tabla 5-9.- Comparativa entre los modos de vibración normalizados estimados mediante el problema simétrico de valores propios y los obtenidos analíticamente (viga continua de tres vanos). .091

Tabla 5-10. Valores medios y desviación típica de los parámetros modales estimados mediante el problema simétrico de valores propios.

Tabla 5-11.- Modos de vibración estimados mediante el problema simétrico de valores propios.

Tabla 5-12.- Modos de vibración normalizados estimados mediante el problema simétrico de valores propios .093

Tabla 5-13. Valores de MAC comparando los modos normalizados obtenidos numéricamente y los experimentales obtenidos mediante el problema simétrico de valores propios.

Tabla 5-14. Comparativa entre los parámetros modales estimados mediante el escalado a partir de la FRF y los obtenidos analíticamente (viga biapoyada). .09

Tabla 5-15.- Comparativa entre los modos de vibración estimados mediante el escalado a partir de la FRF y los obtenidos analíticamente (viga biapoyada). .094 Tabla 5-16.- Comparativa entre los modos de vibración normalizados estimados mediante el escalado a partir de la FRF y los obtenidos analíticamente (viga biapoyada)..............94 Tabla 5-17. Comparativa entre los parámetros modales estimados mediante el escalado a partir de la FRF y los obtenidos analíticamente (viga continua de tres vanos). .09 
Tabla 5-18.- Comparativa entre los modos de vibración estimados mediante el escalado a partir de la FRF y los obtenidos analíticamente (viga continua de tres vanos). .95

Tabla 5-19.- Comparativa entre los modos de vibración normalizados estimados mediante el escalado a partir de la FRF y los obtenidos analíticamente (viga continua de tres vanos).

Tabla 5-20. Valores medios y desviación típica de los parámetros modales estimados mediante el escalado a partir de la FRF. .096

Tabla 5-21. Modos de vibración estimados mediante el escalado a partir de la FRF.....97 Tabla 5-22. Modos de vibración normalizados estimados mediante el escalado a partir de la FRF. .09

Tabla 5-23. Valores de MAC comparando los modos normalizados obtenidos numéricamente y los experimentales empleando el escalado a partir de la FRF. .97 



\section{CAPÍTULO 1. INTRODUCCIÓN}




\subsection{El tema en su contexto}

Debido a la crisis económica que ha sufrido nuestro país en los últimos años, motivada entre otras razones al desplome de la construcción, es necesario realizar una reestructuración del sector como clara apuesta de futuro hacia un alto grado de competitividad. Esta reestructuración debe basarse entre otros pilares en una dinamización innovadora, eliminando las malas prácticas tradicionales que incrementan los costes de la construcción en todas las fases del proyecto, desde su redacción hasta su puesta en servicio y mantenimiento.

Partiendo de este escenario acotamos el área de estudio a un caso particular de estructuras de ingeniería civil, las pasarelas peatonales, que constituyen un paradigma muy claro de elementos en cuyo diseño y construcción se trata de innovar empleando materiales y sistemas constructivos, que resultan en ejemplos más esbeltos, ambiciosos y complejos.

Este hecho, junto con las actuales demandas sociales relativas a la percepción y confort, suscitan la necesidad de integrar el cálculo dinámico de manera sistemática en todas las fases de proyecto (diseño, construcción, puesta en servicio, mantenimiento) ya que estas construcciones son propensas a ser excitadas por las personas que las ocupan, pudiendo los efectos dinámicos producidos inducir, además de problemas de servicio, amplificaciones importantes y repetitivas de los esfuerzos y deformaciones que pueden afectar a la seguridad resistente o a la vida a fatiga de la estructura [1].

Aunque las normativas recogen diversos criterios de evaluación de estos fenómenos, también reflejan la dificultad de evaluar con precisión el comportamiento dinámico de las estructuras, exigiendo en algunos casos un estudio dinámico riguroso.

Por ello es fundamental comprobar la validez de los diferentes métodos y herramientas de análisis y caracterización dinámica, contrastando los resultados obtenidos por varios de ellos para el mismo caso y pudiendo corroborarlos con medidas experimentales. 


\subsection{Motivación}

En la línea presentada en el apartado anterior se redacta la presente tesis, centrándose en la verificación de la validez del análisis de elementos finitos (FEA - Finite Element Analysis) para la obtención de un modelo que simule el comportamiento dinámico en cualquier fase del proyecto y la verificación de dos metodologías para el cálculo de los principales parámetros dinámicos que incluye el escalado de los modos de vibración aplicables en la fase de puesta en servicio. Para ello se empleará como ejemplo una pasarela peatonal actualmente el servicio, la Pasarela del Museo de la Ciencia (PMC) de Valladolid que ya ha sido objeto de un estudio dinámico en la tesis presentada por Jesús de Sebastián Sanz [2].

El fin es contribuir a una mejor comprensión del comportamiento dinámico de estas estructuras y ampliar las herramientas disponibles para su evaluación, intentando corroborar la utilidad de las mismas, verificando su validez con un caso real en servicio de cierta complejidad geométrica, para la proliferación de su uso y ayudar así a los proyectistas y a las administraciones responsables del mantenimiento de este tipo de estructuras.

En el primer caso se ha partido de la premisa de que una de las herramientas de cálculo dinámico con mayor profusión es el método de elementos finitos (FEM - Finite Element Method), ya que los actuales programas de simulación son muy competitivos respecto al ahorro de tiempo y costes. Aunque es una técnica ampliamente empleada en otros ámbitos de la ingeniería, en el caso del cálculo dinámico de estructuras civiles no ha proliferado tanto debido a la existencia de normativas y guías nacionales e internacionales [1, 3-11] que proporcionan metodologías simplificativas, que siendo útiles para la acotación del problema dinámico no ofrecen la enorme versatilidad de este análisis numérico. La Pasarela del Museo de la Ciencia (PMC) de Valladolid, que sirve de modelo para la validación de este análisis en el presente estudio, es un excelente ejemplo de complejidad geométrica que puede ser abordada mediante este instrumento. Como primer paso se modeliza la geometría de la pasarela mediante el programa comercial de elementos finitos ANSYS, posteriormente se ajusta el modelo y se comparan los resultados alcanzados con los obtenidos experimentalmente procedentes de un estudio dinámico anterior. La etapa de diseño es decisiva tanto para los costes como para la calidad del proyecto y el modelo 
que se genera, una vez validado, nos permitirá estudiar los diferentes factores que provocan la susceptibilidad a las vibraciones de la pasarela para, por ejemplo, contemplar diferentes alternativas de diseño y/o predecir su comportamiento ante diferentes solicitaciones durante las diversas fases del proyecto. Asimismo se puede emplear el conocimiento generado en el presente estudio en las fases de diseño de otros casos similares, reduciendo de este modo las inversiones que serían necesarias implantar para resolver problemas de efectos dinámicos no deseados en etapas más tardías que habitualmente suponen costes mucho más elevados. Se deja estos últimos planteamientos para futuras líneas de investigación.

Como segunda parte del planteamiento de la presente tesis, tomando como datos iniciales los registros de aceleración generados en el Análisis Modal Experimental tradicional (EMA - Experimental Modal Analysis), se procede a la validación de dos metodologías que nos permiten identificar los parámetros modales más relevantes que caracterizan el comportamiento dinámico del mismo. Dichas metodologías parten del método de identificación más utilizado en la actualidad, el Stochastic Subspace Identification (SSI), incorporando técnicas adicionales que nos permitan estimar todos los parámetros modales, incluidos las masas modales de cada modo de vibración, ya que dicho método de identificación no permite la obtención directa de las mismas y el modelo resulta incompleto lo que no permite la simulación dinámica del sistema.

Para ello, en primer lugar partiendo de la representación del sistema dinámico en el espacio de estados mediante la técnica SSI se escalan los modos de vibración mediante dos procesos, el primero de ellos resolviendo un problema simétrico de valores propios cuyo requisito mínimo es que en todos los grados de libertad se debe ubicar o bien un sensor o un actuador con al menos una localización de ubicación conjunta con par sensoractuador y el segundo partiendo de los residuos de la descomposición en fracciones parciales de la función respuesta en frecuencia (FRF) de las mediciones. 


\subsection{Antecedentes y estado actual}

Respecto al Método de los Elementos Finitos (FEM, en inglés Finite Element Method), su origen moderno se remonta a principios del siglo XX cuando algunos investigadores, como Hrennikoff y McHenry, aproximaron y modelizaron un sólido elástico con sección transversal continua usando un conjunto discreto de barras elásticas equivalente $[12,13]$. Sin embargo, se considera que Courant fue la primera persona en desarrollar un método de elementos finitos en 1943. En una publicación a principios de los cuarenta, Courant propone la utilización de funciones polinómicas para la formulación de problemas elásticos en subregiones triangulares, como un método especial del método variacional de Rayleigh-Ritz para aproximar soluciones [14].

El siguiente paso significativo en el campo de la ingeniería estructural, fue dado por Levy en 1953 [15] que desarrolló el método de rigidez y desplazamiento, sin embargo, sus ecuaciones requerían demasiado trabajo para su solución a mano por lo que este método de análisis numérico no se popularizó hasta el desarrollo de las computadoras.

Entre 1954 y 1955, Argyris y Kelsey [16] emplearon métodos energéticos para el desarrollo del análisis estructural por medio de matrices, lo que marcó una tendencia en la evolución del análisis por elementos finitos (FEA, en inglés Finite Element Analysis). En sus artículos se presenta completamente desarrollada la formulación matricial de la teoría de estructuras y donde están claramente perfilados los métodos de las fuerzas y los desplazamientos.

La que se considera la primera aplicación del FEM fue un trabajo realizado por Clough en la Boeing Airplane Company dentro de la unidad de dinámica estructural, dirigido por Turner, para la evaluación de la rigidez del ala delta de un avión. Puesto que los modelos de barras no daban buenos resultados, Turner sugirió la división de la superficie en porciones triangulares cuya rigidez fue evaluada utilizando el teorema de Castigliano suponiendo estados constantes de tensión normal y cortante en los triángulos y obteniendo la rigidez total sumando adecuadamente las de cada triángulo (método directo de rigidez). Se presentó una comunicación sobre este trabajo en la reunión del Institute of Aeronautical Sciences en enero de 1954 en Nueva York, firmada por Turner, Clough, Martin y Topp con el título "Stiffness and deflection analysis of complex structures" 
(aunque se publicó en 1956). Sin embargo no fue hasta los 60 cuando Clough hizo popular el término "elemento finito", con la primera comunicación en la que figura dicho término publicada en la American Society of Civil Engineers (ASCE) con el título "The Finite Element method in plane stress analysis" [17].

A partir de los años 60, se producen numerosos avances que permitieron el rápido desarrollo de este método como estudios relativos a la matriz de masa, grandes desplazamientos, problemas no lineales, o la aplicación a problemas de tres dimensiones, pandeo, viscoelasticidad, ...[18-27]. Asimismo se produce un gran interés entre los matemáticos por la solución de ecuaciones diferenciales lineales y no lineales mediante el FEM lo que permitió que se empezara a aplicar en otras áreas de la ingeniería, como la transferencia de calor o problemas de flujo de fluidos [28-33]. Zienkiewicz and Cheung escribieron el primer libro dedicado por completo al FEM en 1967 [34] en el que se presenta una interpretación amplia de este método y su aplicación a diferentes campos.

A mediados de los setenta, Belytschko [35, 36] mejoró las técnicas numéricas aplicables a la resolución de los sistemas de ecuaciones de problemas dinámicos no lineales con grandes desplazamientos.

Es durante esta década cuando se considera que el FEM alcanza la madurez, apareciendo los grandes programas comerciales. En 1971, el programa ANSYS se lanza al mercado por primera vez.

Desde entonces hasta nuestros días se han generado una gran cantidad de artículos y publicaciones que demuestran que el FEM es una de las herramientas más potentes y probadas para la solución de problemas de ingeniería y que amplía su campo de aplicación a medida que se realizan más estudios validando dicho método, como sería el caso de la dinámica de las pasarelas peatonales.

Sin embargo, las predicciones efectuadas con el uso de modelos analíticos difieren del comportamiento de la estructura real obtenidas mediante ensayos experimentales. Como respuesta a dicho problema apareció el FEM Updating (también denominado ajuste o actualización de los modelos de elementos finitos) que tiene como objetivo efectuar correcciones en los parámetros de los modelos de elementos finitos mejorando las suposiciones de los modelos y aproximando sus propiedades a las de la estructura real. 
La retroalimentación entre las mediciones de la estructura y los procedimientos analíticos permiten obtener mejores modelos numéricos.

La extensa investigación que se ha realizado en las últimas décadas sobre el FEM Updating nos indica que aún no ha alcanzado su madurez y que no existe ningún procedimiento general ni completamente fiable que pueda ser formulado para todos los casos. Algunos autores [37-43] han publicado y comparado varias técnicas pero no se alcanza ningún acuerdo sobre métodos o prácticas. En la mayoría de los casos la solución parece depender de los parámetros y constantes seleccionados así como de la técnica empleada.

En el caso del análisis modal experimental, se puede considerar que el comienzo de su era moderna se produce a partir de la mitad de la década de los sesenta cuando la teoría ya se había desarrollado y el hardware en términos de sensores y equipos de medición estaba disponible a nivel comercial.

Actualmente se ha convertido en una de las metodologías de cálculo dinámico de estructuras más importantes y se estructura en tres fases principales: recolección de datos, identificación del sistema y por último estimación de los parámetros modales [44]. Es la segunda etapa, la de identificación del sistema, la que juega un papel crucial en el número y tipo de parámetros modales que se pueden estimar [45]. Los métodos de identificación tradicionales o clásicos se basan en aplicar una excitación al sistema y a continuación, registrar la correspondiente respuesta (EMA) [46]. Si los ensayos se realizan en condiciones de uso, en los que la magnitud de las fuerzas excitadoras es desconocida, el análisis se denomina análisis modal operacional (OMA - Operational Modal Analysis) y si los ensayos se llevan a cabo en condiciones de operación aplicando una o varias fuerzas artificiales se denomina análisis modal operacional con entradas exógenas (OMAX Operacional Modal Analysis with eXogenous inputs).

El método de identificación más utilizado en la actualidad es la técnica de identificación en el subespacio estocástico (abreviado SSI, de su denominación en inglés). Este método se ha aplicado en muchas situaciones para extraer los parámetros modales de estructuras existentes, por ejemplo puentes y edificios, solicitadas por la vibración ambiental. Sin embargo, la información modal que se obtiene de estos métodos de identificación, es 
incompleta ya que proporciona estimaciones de frecuencias, factores de amortiguamiento y modos de vibración asociados, pero dichos modos no están normalizados respecto de la matriz de masa o, dicho de otra forma, esos métodos no calculan las masas generalizadas correspondientes a cada uno de los modos estimados [47].

Para resolver este problema, se han desarrollado algunos métodos de escalado de los modos de vibración que se basan en la actualización de un modelo de elementos finitos de la estructura empleando los parámetros modales estimados en el OMA [42, 48]. También se han desarrollado soluciones basadas en la suposición de que la matriz de masa de la estructura (o su inversa) es conocida [49] o modificando el comportamiento dinámico de la estructura variando la masa o la rigidez y realizando un análisis modal operacional tanto en la estructura original como en la modificada [50-56], lo que requiere un procedimiento experimental extenso al emplear los parámetros modales de la estructura original y también de la modificada.

Otros métodos de escalado consisten en añadir sistemas de vibración secundarios, midiendo y procesando la respuesta conjunta [44].

La identificación de los parámetros en una formulación diferencial de primer orden también ha recibido considerable atención, pero como es sabido, si se parte de un espacio de estados modelo, y se trata de identificar los parámetros del modelo de segundo orden, cuestiones como la no unicidad de la solución complica la resolución del problema inverso [57-63]. Sin embargo se han desarrollado varias metodologías que resuelven el problema imponiendo diferentes restricciones en el número de sensores y actuadores empleados [47, 64-67]. 


\subsection{Objetivos}

El principal objetivo de este trabajo es validar diferentes métodos y herramientas de caracterización modal, aplicándolos a un caso real (una estructura actualmente en servicio) y complementando un examen dinámico anterior realizado sobre la misma. De esta forma se amplía el abanico de metodologías que faciliten la implantación del estudio de las vibraciones en las estructuras de obra civil, permitiendo que este tipo de acciones no limiten la progresiva evolución que se está desarrollando en el diseño de las mismas. Por otra parte, dicha validación no se limita exclusivamente a las etapas de puesta en servicio y mantenimiento, ya que se analiza una de las herramientas de mayor profusión, el FEA, en las fases más tempranas del proyecto con el objetivo de que su implementación en el diseño de este tipo de estructuras permita efectuar modificaciones o correcciones al menor coste posible.

Concretando, los objetivos de la tesis han sido los siguientes:

- Estudios sobre la Pasarela del Museo de la Ciencia de Valladolid:

- Modelización de la estructura completa mediante el software de Elementos Finitos ANSYS.

- Ajuste del modelo (FEM Updating) mediante la comparación de las frecuencias y formas modales, con los resultados experimentales obtenidos en un estudio anterior.

- Análisis modal y dinámico de la Pasarela del Museo de la Ciencia de Valladolid:

- Validación de una metodología para estimar los parámetros modales del sistema dinámico, partiendo de una representación en el espacio de estados mediante la técnica SSI e identificando mediante la adecuada matriz de transformación, los parámetros físicos del sistema (matrices de masa, amortiguamiento y rigidez) que permiten obtener las masas modales y/o modos normalizados respecto a la matriz de masa.

- Validación de una segunda metodología en la que partiendo de nuevo de una representación en el espacio de estados mediante la técnica SSI, se 
escalan los modos de vibración a partir de la estimación de los residuos de la descomposición en fracciones parciales de la FRF de las mediciones. 


\subsection{Herramientas}

\section{ANSYS}

ANSYS [68] es un programa de análisis y diseño basado en el método de cálculo por elementos finitos. ANSYS structural analysis software permite resolver problemas complejos de ingeniería estructural. Con las herramientas de FEA se pueden personalizar y automatizar simulaciones y parametrizarlas para analizar múltiples escenarios de diseño.

El programa ANSYS [69] tiene dos niveles básicos: el nivel de inicio y el nivel de procesamiento. Cuando se entra en el programa, nos encontramos en el nivel de inicio y desde este nivel se puede acceder a uno de los procesadores de ANSYS: PREP7, SOLUTION, POST1, etc. Un procesador es una colección de funciones y rutinas que sirven para propósitos específicos.

PREP7, denominado pre-procesador, contiene los comandos necesarios para construir un modelo:

- define tipos de elementos y opciones

- define constantes reales de los elementos

- define las propiedades de los materiales

- crea el modelo geométrico

- define los controles de mallado

- malla los objetos creados.

El procesador SOLUTION tiene los comandos que permiten aplicar las condiciones de contorno y las cargas. Una vez que se proporciona toda la información a este procesador, éste resuelve el problema.

POST1, denominado post-procesador, contiene los comandos que permiten listar y mostrar los resultados del análisis:

- leer resultados de un archivo de resultados

- leer los resultados de los elementos 
- dibujar/mostrar en gráficos los resultados

- listar los resultados

\section{MATLAB}

MATLAB [70] es un entorno de cálculo técnico de altas prestaciones para cálculo numérico y visualización. Integra:

- Análisis numérico

- Cálculo matricial

- Procesamiento de señales

- Gráficos

MATLAB se utiliza para investigación y resolución de problemas prácticos de ingeniería y matemáticas, con un gran énfasis en aplicaciones de control y procesamiento de señales. MATLAB también proporciona una serie de soluciones específicas denominadas TOOLBOX, que son conjuntos de funciones MATLAB que extienden el entorno para resolver clases particulares de problemas como:

- Procesamiento de señales

- Diseño de sistemas de control

- Simulación de sistemas dinámicos

- Identificación de sistemas

- Redes neuronales y otros

Las prestaciones más importantes de MATLAB son:

- Escritura del programa en lenguaje matemático

- Implementación de las matrices como elementos básicos del lenguaje, lo que permite una gran reducción del código, al no necesitar implementar el cálculo matricial.

- Implementación de aritmética compleja.

- Un gran contenido de órdenes específicas, agrupadas en TOOLBOXES. 
- Posibilidad de ampliar y adaptar el lenguaje, mediante ficheros de script y funciones .m.

\section{- MATHEMATICA:}

Mathematica [71] es un programa para la computación y visualización numérica, simbólica y gráfica y que ofrece una herramienta interactiva de cálculo y un lenguaje de programación potente:

- Una calculadora de tipo numérico. La diferencia con una calculadora es que tiene implementadas aproximadamente unas 750 funciones y además trabaja con la precisión que se desee (incluyendo precisión infinita).

- Un paquete de subrutinas para cálculo matemático. Se pueden hacer operaciones que requieran el uso de funciones o de procedimientos especiales como la integración numérica, la optimización de funciones, programación lineal, etc. que se pueden utilizar directamente.

- Una calculadora simbólica. Con la posibilidad de trabajar con expresiones simbólicas. Se puede definir una función que quedará almacenada tal como es, y no en forma de algoritmo que pueda dar aproximaciones a la función. Se pueden sustituir valores de la variable como expresiones, parámetros, etc. y el sistema entiende y opera en forma simbólica (exacta).

- Una potente herramienta de cálculo simbólico. Se puede derivar e integrar funciones, resolver ecuaciones diferenciales, calcular límites, manipular series de potencias, utilizar transformadas integrales, ...

- Un paquete gráfico. Permite dibujar en dos o tres dimensiones, elegir perspectivas, sistemas de representación, sistemas de coordenadas, animar las gráficas, ...

- Un lenguaje de programación, se puede realizar programación a tres niveles:

- Programación de tipo procedural (uso de bloques, iteraciones y ciclos, recursividad, etc.).

- Programación funcional (con la posibilidad de definir funciones, operadores funcionales, etc.). 
- Programación basada en reglas (suministrando reglas que indican como operar o transformar expresiones simbólicas, funciones, etc.).

- Un sistema para crear documentos interactivos, con posibilidad de incluir texto, gráficos, sonidos, animaciones, etc.

- Un sistema de apoyo a otros programas. Se puede comunicar con Mathematica desde otros programas y pedirles tareas que realizará y después enviará los resultados.

El programa Mathematica constituye una herramienta muy potente para la realización de todo tipo de cálculos matemáticos: operaciones aritméticas, cálculo simbólico, gráficos... El programa Mathematica está estructurado en 2 partes:

- El Front End: es la interface que permite la comunicación con el usuario. Presenta las entradas y salidas y permite crear y editar ficheros que contienen texto, cálculos, gráficas, etc. Estos ficheros tienen la extensión .nb y se denominan Notebooks (Cuadernos de notas). La información que contienen estos ficheros se estructura mediante un sistema de celdas de diferentes tipos (título, subtítulo, sección, texto, input, output, ....) .

- El Kernel (núcleo): constituye la estructura interna del programa y es el que se encarga de la realización de los cálculos y operaciones matemáticas que solicita el usuario. 


\subsection{Contenido}

En el primer capítulo se presenta el tema en su contexto haciendo especial hincapié en la importancia que tiene ampliar las herramientas disponibles para la caracterización dinámica de las pasarelas, intentando corroborar la utilidad de las mismas, verificando su validez con un caso real en servicio de cierta complejidad geométrica, para contribuir a la proliferación de su uso. También se realiza una descripción de los trabajos desarrollados por otros autores respecto al tema en cuestión y se indican los objetivos específicos de esta Tesis Doctoral.

En los siguientes capítulos se hace una síntesis de la metodología empleada y se estructura siguiendo el proceso en que se han desarrollado los trabajos conducentes al presente documento. En el segundo capítulo se resumen conceptos básicos de vibraciones y los principales aspectos que contribuyen a una mejor compresión del análisis modal, que es la base de las diferentes metodologías propuestas, así como un resumen de las técnicas de análisis modal aplicadas a la Pasarela del Museo de la Ciencia (objeto de estudio del presente documento). Los registros, y los resultados experimentales obtenidos en base a ellos en un estudio anterior, son los que se emplean para comprobar la validez de las técnicas descritas en los sucesivos apartados. En el capítulo 3 se realiza una sinopsis del Método de los Elementos Finitos y del FEM Updating y en el capítulo 4 se explica el desarrollo matemático en que se fundamentan las dos metodologías de caracterización modal que adicionalmente se proponen.

Posteriormente en el quinto capítulo se recopila el proceso de modelización de la pasarela peatonal mediante el software de elementos finitos ANSYS y los resultados logrados, así como la validación y aplicación de las técnicas de escalado propuestas y los parámetros modales obtenidos.

En el capítulo 6 se muestran las conclusiones extraídas de este trabajo y se establecen las líneas futuras de investigación.

Por último, en el Anexo se incluyen diversas publicaciones realizadas durante el desarrollo de la presente Tesis. 

CAPÍTULO 2. ANÁLISIS MODAL 
El análisis modal es un proceso mediante el cual se describe una estructura en términos de sus propiedades dinámicas o parámetros modales que son la frecuencia, el amortiguamiento y los modos de vibración, para todos los modos en el rango de frecuencias de interés. Todas las estructuras poseen frecuencias naturales y modos de vibración, que dependen básicamente de la masa y de la rigidez de la estructura. En el diseño es necesario identificar estas frecuencias y conocer cómo afectan a la respuesta de la estructura cuando una fuerza actúa sobre la misma. El análisis modal es una herramienta eficiente para describir, comprender y modelar el comportamiento de las estructuras.

El análisis modal parte de la hipótesis de considerar que la respuesta en vibración de un sistema puede ser expresada como una combinación de una serie de movimientos armónicos simples llamados modos naturales de vibración, intrínsecos al sistema y determinados por el valor y distribución de su masa, rigidez y amortiguamiento. Cada modo se define a partir de sus parámetros modales: frecuencia natural, amortiguamiento modal y forma característica de desplazamiento. El grado de participación de cada modo en el total de la vibración viene determinado por las características de la excitación que actúa sobre el sistema y por las formas de los modos.

De forma simplificada se puede decir que en el análisis modal una deformación compleja de una estructura se descompone en una serie de modos simples de deformación con parámetros de frecuencia y amortiguamiento individuales. Su fin último es la construcción de un modelo modal del comportamiento dinámico de la estructura. El procedimiento consiste en la adquisición de datos, su análisis y luego determinar todos los parámetros modales.

La forma teórica del análisis modal consiste en plantear la ecuación del movimiento, suponer una forma de la respuesta e imponer que esta cumpla la ecuación que gobierna el movimiento del sistema, lo que supone resolver un problema de autovalores y autovectores. Para obtener la ecuación de movimiento es necesario calcular las matrices de masa y rigidez y los factores de amortiguamiento.

Hoy en día, el análisis teórico suele hacerse mediante modelos discretos (generalmente por elementos finitos) con un mayor o menor grado de idealización. 
En el caso del análisis modal experimental no es preciso el cálculo de estas matrices. La identificación modal experimental se consigue a partir de mediciones del movimiento (desplazamientos, velocidades o aceleraciones) en diferentes localizaciones del sistema (p. ej. estructura). Tres aspectos fundamentales del análisis modal experimental son: la instrumentación de la estructura, la adquisición de señales/registros y el tratamiento de señales.

El análisis modal tiene muchas utilidades. Algunas de las más importantes se indican a continuación [72]:

- comprender como se comportan las estructuras bajo la acción de fuerzas dinámicas,

- medir las propiedades de la estructura cuando se somete a una excitación para refinar y validar modelos analíticos. Cada vez se usan más los ensayos dinámicos combinados con los análisis de elementos finitos para mejorar los modelos analíticos a partir de los cuales se pueden identificar los parámetros que definen las secciones, el módulo de Young del material, condiciones de apoyo, etc,

- desarrollar un modelo, ya que proporciona una descripción definitiva del comportamiento de una estructura que puede ser evaluada para ciertas especificaciones del diseño,

- controlar la integridad de cierta estructura y su comportamiento, con el fin de detectar problemas y evitarlos,

- comprobar el estado de las estructuras después de sufrir daños como por ejemplo los debidos a un terremoto,

- ayudar en el diseño de todo tipo de estructuras y hacer simulaciones en el desarrollo de prototipos,

- predecir o simular la respuesta a excitaciones externas o cómo se comportará la estructura bajo otras condiciones de operación diferentes,

- simular cambios en las características dinámicas, debido a modificaciones físicas, bien sea añadiendo una carga mayor o una rigidez para obtener una propiedad dinámica deseada,

- estimar las fuerzas que actúan sobre la estructura,

- hacer un análisis del ensamblaje de estructuras. 
En los apartados 2.1 y 2.2 se resumen conceptos básicos de la teoría del análisis modal y del análisis modal experimental a modo de repaso y para introducir la nomenclatura de forma natural. En el apartado 2.3 se recapitulan las técnicas de análisis modal aplicadas a la Pasarela del Museo de la Ciencia, objeto de estudio del presente documento. 


\subsection{Teoría del análisis modal}

El oscilador armónico simple es una estructura con un único grado de libertad caracterizada por su masa, rigidez y su capacidad de disipación de energía mecánica (amortiguamiento) [73].

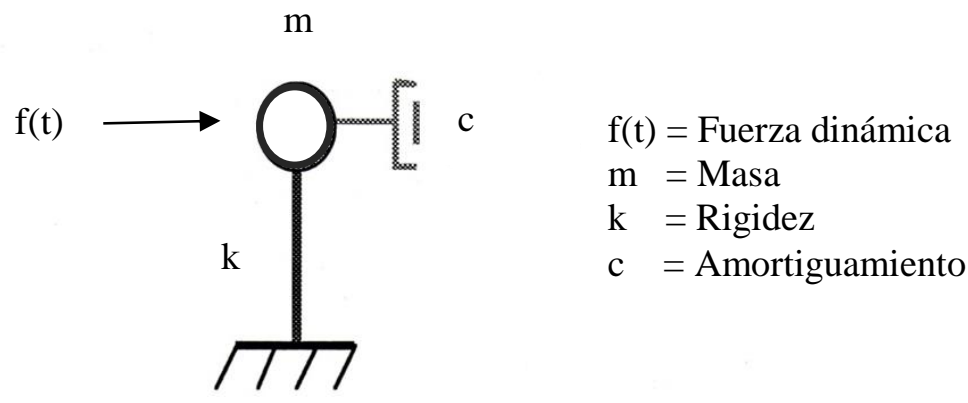

Figura 2-1. Oscilador armónico simple

Sobre la masa del oscilador actúan la fuerza exterior de carácter dinámico f(t), la fuerza elástica fe(t) que es proporcional a la rigidez de la estructura y a su desplazamiento, la fuerza de inercia fi(t) - producto de la masa por la aceleración- y la fuerza de amortiguamiento fa(t) o de disipación de energía mecánica que, en general, se supone proporcional a la velocidad (amortiguamiento viscoso). Todas las fuerzas que actúan sobre el oscilador deben estar en equilibrio y, por tanto, su suma debe ser nula. Esta ecuación de equilibrio (ecuación de D’Alembert) se puede formular del modo siguiente:

$$
f i(t)+f a(t)+f e(t)=f(t) \rightarrow m \ddot{x}(t)+c \dot{x}(t)+k x(t)=f(t)
$$

donde las notaciones (") y (') representan la primera y segunda derivada respecto al tiempo y:

$x(t) \quad$ Desplazamiento respecto a la posición de equilibrio

$m \quad$ Masa del oscilador

c Coeficiente de amortiguamiento

$k \quad$ Rigidez

$f(t) \quad$ Fuerza exterior aplicada

Un sistema de $\mathrm{N}$ grados de libertad (NGDL) es aquél que precisa de $\mathrm{N}$ parámetros o coordenadas para que su posición y configuración deformada quede definida. Por regla 
general, aunque no siempre, se suelen tomar como coordenadas del sistema los desplazamientos de un conjunto de puntos llamados nudos/nodos.

$\mathrm{Si}$ consideramos todos aquellos grados de libertad necesarios para reproducir el comportamiento de la estructura, la ecuación de equilibrio se formula para cada uno de los N GDL considerados y, mediante procedimientos de ensamblaje similares a los del cálculo matricial estático, el conjunto de ecuaciones de equilibrio se puede expresar como un sistema matricial de orden $\mathrm{N}$ del modo siguiente:

$$
\boldsymbol{M} \ddot{\boldsymbol{x}}(t)+\boldsymbol{C} \dot{\boldsymbol{x}}(t)+\boldsymbol{K} \boldsymbol{x}(t)=\boldsymbol{f}(t)
$$

donde la notación hace referencia a las siguientes magnitudes:

$\boldsymbol{x}(t) \quad$ Vector desplazamiento respecto a la posición de equilibrio

$\dot{\boldsymbol{x}}(t) \quad$ Primera derivada respecto al tiempo del vector desplazamiento

$\ddot{x}(t) \quad$ Segunda derivada respecto al tiempo del vector desplazamiento

M Matriz de masas

C Matriz de amortiguamiento

K Matriz de rigidez

$\boldsymbol{f}(t) \quad$ Vector de fuerzas exteriores

\subsubsection{Frecuencia natural}

En el caso particular de la ecuación diferencial de movimiento para un oscilador simple sin amortiguamiento - sistema de un solo grado de libertad, sin amortiguamiento y libre de la acción de fuerzas exteriores durante su movimiento vibratorio [75],

$$
m \ddot{x}(t)+k x(t)=f(t)
$$

al tratarse de una ecuación diferencial lineal de $2^{\circ}$ orden, la solución es de la forma:

$$
x(t)=A \cdot \cos \omega t+B \cdot \operatorname{sen} \omega t
$$


donde A y B son constantes que dependen de las condiciones iniciales, mientras que $\omega$ es un valor que denota una propiedad física del sistema, la frecuencia natural expresada en $\mathrm{rad} / \mathrm{s}$.

$$
\omega=\sqrt{\frac{k}{m}}
$$

Siendo
$k$
la rigidez del sistema
$m$
la masa del sistema

Aplicando las condiciones iniciales

$t=t_{0} \quad x=x_{0} \quad y \quad x^{\prime}=v_{0}$

obtenemos $x_{0}=A \quad y v_{0}=B \omega$

quedando finalmente

$$
x(t)=x_{0} \cos \omega t+v_{0} / \omega \operatorname{sen} \omega t
$$

En el caso de este movimiento, que es armónico y por lo tanto periódico, el periodo T del movimiento está determinado por $T=2 \pi / \omega$.

El valor recíproco del periodo es la frecuencia natural $f=1 / T=\omega / 2 \pi$ expresada en hercios o ciclos por segundo.

Cada estructura tiene tantas frecuencias naturales y modos asociados de vibración como grados de libertad. Estos modos se clasifican por la cantidad de energía que se activa en la oscilación. Por lo tanto, la primera frecuencia natural es la que representa un nivel inferior de energía y por lo tanto es la que se activa con mayor probabilidad.

\subsubsection{Amortiguamiento}

El oscilador simple una vez excitado oscila a su frecuencia natural indefinidamente, con una amplitud constante. La experiencia nos indica sin embargo que no es posible tener un mecanismo que vibre en estas condiciones ideales. Fuerzas de fricción o de amortiguamiento están siempre presentes en cualquier sistema en movimiento, estas 
fuerzas disipan energía. En otras palabras, la presencia inevitable de estas fuerzas de fricción constituye un mecanismo por el cual la energía mecánica del sistema, energía cinética o potencial se disipa en forma de otros tipos de energía como el calor. El mecanismo de esta transformación o disipación de energía es bastante complejo y en la actualidad no se comprende totalmente. Para considerar estas fuerzas de disipación en el análisis de sistemas dinámicos es necesario introducir simplificaciones en cuanto a su naturaleza, basadas en la experiencia.

Cuando se consideran las fuerzas de amortiguamiento o fricción en el análisis dinámico de estructuras, generalmente se asume que éstas son proporcionales a la magnitud de velocidad y opuestas a la dirección del movimiento. Este tipo de fricción se conoce como amortiguamiento viscoso. La razón principal del uso generalizado de esta suposición es que conduce a un análisis matemático relativamente simple, aunque no se corresponde exactamente con lo que ocurre en las estructuras.

La ecuación diferencial del movimiento en el caso del oscilador simple con amortiguamiento viscoso sería [75]:

$$
m \ddot{x}(t)+c \dot{x}(t)+k x(t)=0
$$

siendo una solución que satisface esta ecuación

$$
x=C e^{p t}
$$

La aplicación de esta función en la ecuación da:

$$
m C p^{2} e^{p t}+c C p e^{p t}+k C e^{p t}=0
$$

si eliminamos factores comunes

$$
m p^{2}+c p+k=0
$$

las raíces de esta ecuación son:

$$
\begin{aligned}
& p_{1}=-\frac{c}{2 m}+\sqrt{\left(\frac{c}{2 m}\right)^{2}-\frac{k}{m}} \\
& p_{2}=-\frac{c}{2 m}-\sqrt{\left(\frac{c}{2 m}\right)^{2}-\frac{k}{m}}
\end{aligned}
$$

(Nótese que $k / m=\omega^{2}$ ) 
Por lo tanto, la solución general de la ecuación viene dada por la superposición de las dos soluciones posibles

$$
x(t)=C_{1} e^{p_{1}^{t}}+C_{2} e^{p_{2} t}
$$

Donde $\mathrm{C}_{1}$ y $\mathrm{C}_{2}$ son constantes de integración que se determinan a partir de las condiciones iniciales.

La solución general depende de los valores de amortiguamiento $c$, de la masa $m$ y de la rigidez $k$, que determinan el signo de la raíz cuadrada, distinguiéndose tres posibles situaciones [73]:

\section{Sistemas Críticos:}

$$
(c / 2 m)^{2}-\omega^{2}=0
$$

El valor de c, solución de la ecuación, se denomina amortiguamiento crítico $c_{c}$ :

$$
c_{c}=2 m \omega
$$

Los sistemas críticos marcan el límite entre la solución oscilante respecto a la posición de equilibrio y aquellos en los que el sistema no oscila y, por tanto, la masa desplazada inicialmente de su posición de equilibrio vuelve a dicha posición sin oscilaciones. Nótese que el valor del amortiguamiento crítico de un sistema sólo depende de su masa y rigidez.

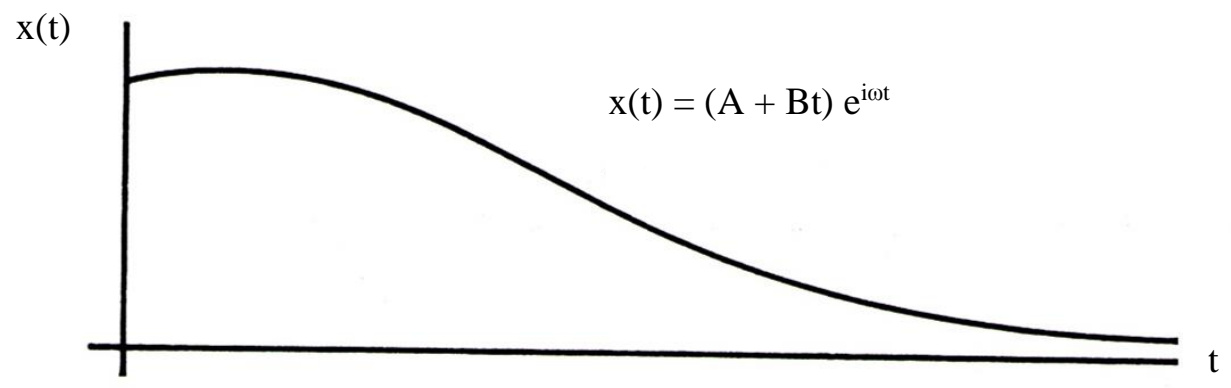

Figura 2-2. Movimiento de un sistema crítico

\section{Sistemas Supercríticos:}

$$
(c / 2 m)^{2}-\omega^{2}>0 \rightarrow c>c_{c}
$$

Los sistemas supercríticos son aquellos en los que el amortiguamiento del sistema supera al valor del amortiguamiento crítico $y$, en consecuencia, el sistema, con unas 
determinadas condiciones iniciales de velocidad y desplazamiento, vuelve a su posición de equilibrio sin oscilar.

\section{Sistemas Subcríticos:}

$$
(c / 2 m)^{2}-\omega^{2}<0 \rightarrow c<c_{c}
$$

Los sistemas subcríticos son aquellos en los que el amortiguamiento del sistema es inferior al crítico y, por tanto, el sistema, a partir de unas determinadas condiciones iniciales de velocidad y desplazamiento, oscila respecto a la posición de equilibrio con amplitudes que van disminuyendo de forma exponencial

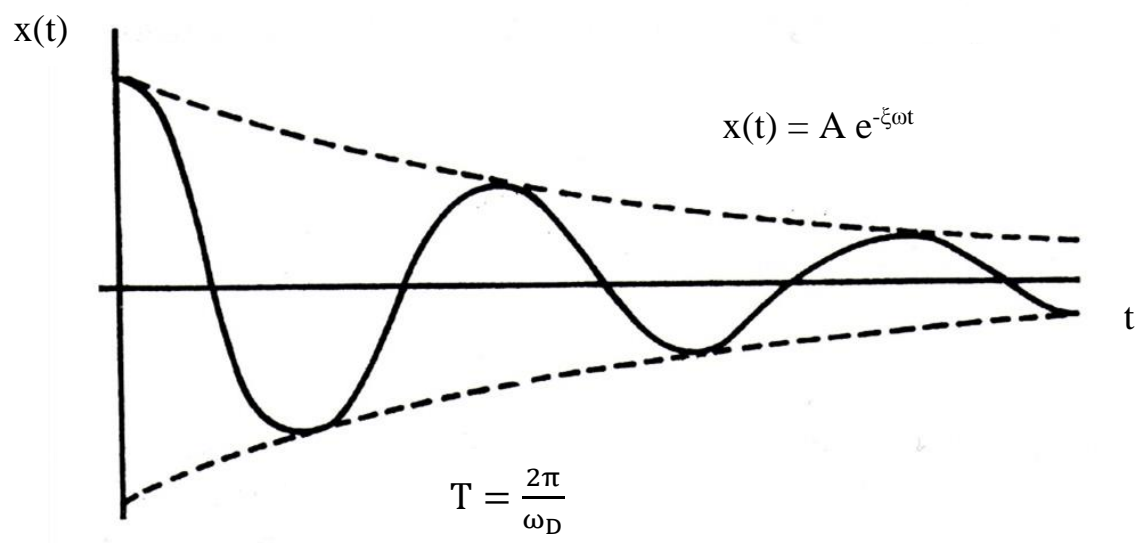

Figura 2-3. Movimiento de un sistema subcrítico

En este caso el valor de $A=x_{0} \cos \omega_{D} t+\left(\frac{v_{0}+x_{0} \xi \omega}{\omega_{D}}\right) \operatorname{sen} \omega_{D} t$

Siendo

$x_{0}=$ desplazamiento inicial

$v_{0}=$ velocidad inicial

$\xi=$ coeficiente o razón de amortiguamiento $=c / 2 \mathrm{m \omega}=c / c_{c}$

$\omega_{D}=$ frecuencia circular amortiguada

$$
\omega_{D}=\omega \sqrt{\left(1-\xi^{2}\right)}
$$


Los valores habituales de amortiguamiento en estructuras varían entre $0,2 \%$ y $5 \%$ del amortiguamiento crítico $c_{c}$. Por este motivo, el valor de la frecuencia natural amortiguada $\omega_{D}^{2}=\omega^{2}\left(1-\xi^{2}\right)$ es prácticamente igual al de la frecuencia natural del sistema sin amortiguamiento $\omega_{D}=\omega$, de forma análoga se puede expresar para el periodo de la oscilación.

\subsubsection{Resonancia}

En muchas ocasiones el movimiento de estructuras se idealiza como sistemas de un solo grado de libertad con excitación armónica, o sea estructuras sujetas a fuerzas o desplazamientos cuyas magnitudes pueden ser representadas por una función seno o coseno, del tiempo. Este tipo de excitación produce uno de los movimientos más importantes en el estudio de las vibraciones mecánicas, así como en aplicaciones de dinámica estructural.

En el caso de excitación armónica, es decir, $f(t)=F_{0} \operatorname{sen} \Omega t$, donde,

$F_{o}=$ amplitud máxima

$\Omega=$ frecuencia de la fuerza

la solución de la ecuación del movimiento en sistemas sin amortiguamiento viene dada por la superposición de dos términos armónicos de frecuencia diferentes

$$
x(t)=\frac{F_{0}}{k} \frac{(\operatorname{sen} \Omega t-\operatorname{sen} \omega t)}{\left(1-r^{2}\right)}
$$

siendo

$r=\Omega / \omega$

En casos reales, las fuerzas de amortiguamiento estarán siempre presentes en el sistema y harán que el segundo término, con la frecuencia natural $\omega$, desaparezca eventualmente. Por esta razón, este término se denomina respuesta transitoria. El término con la frecuencia forzada $\Omega$ en la ecuación se conoce con el nombre de respuesta estacionaria. Es evidente que en el caso de un sistema sin amortiguamiento, la componente transitoria de la solución no desaparece y por lo tanto, la respuesta está dada por los dos términos de la ecuación. Se puede observar también que cuando la frecuencia forzada $\Omega$ es igual a la frecuencia natural $\omega(r=\Omega / \omega=1)$, la amplitud del movimiento tiende a infinito. Un 
sistema que actúa bajo una excitación externa con una frecuencia forzada $\Omega$ que coincide con la frecuencia natural $\omega$ se dice que está en resonancia.

La ecuación diferencial del movimiento para el caso de excitación armónica en sistemas con amortiguamiento (en el caso subcrítico $\mathrm{c}<\mathrm{c}_{\mathrm{r}}$ ) [76]

$$
m \ddot{x}(t)+c \dot{x}(t)+k x(t)=F_{0} \operatorname{sen} \Omega t
$$

tiene como solución la siguiente

$$
x(t)=e^{-\xi \omega t}\left(A \cos \omega_{D} t+B \operatorname{sen} \omega_{D} t\right)+y_{s t} \frac{\operatorname{sen}(\Omega t-\theta)}{\sqrt{\left(1-r^{2}\right)^{2}+(2 r \xi)^{2}}}
$$

recordando que $\omega_{D}$ es la frecuencia con amortiguamiento y $r$ la razón entre frecuencias, siendo

$$
\tan \theta=\frac{c \Omega}{\left(k-m \Omega^{2}\right)}=\frac{2 \xi r}{\left(1-r^{2}\right)}
$$

el desfase presente entre la excitación y la respuesta del sistema, y

$$
y_{s t}=F o / k
$$

el desplazamiento estático, esto es, el desplazamiento que tendría el sistema si la carga fuera aplicada estáticamente, es decir, con frecuencia nula.

Finalmente A y B son constantes de integración que deben ser calculadas a partir de las condiciones iniciales.

El primer sumando se denomina respuesta transitoria y el segundo término respuesta estacionaria y puede verse que la presencia del factor exponencial $\mathrm{e}^{-\xi \omega t}$ hará que esta componente desaparezca con el tiempo dejando el movimiento estacionario.

Se conoce como amplificación dinámica $D$ al siguiente término [77]

$$
D=\frac{1}{\sqrt{\left(1-r^{2}\right)^{2}+(2 r \xi)^{2}}}
$$

y varía con la razón de frecuencias $r$ y con la razón de amortiguamiento $\xi$. 


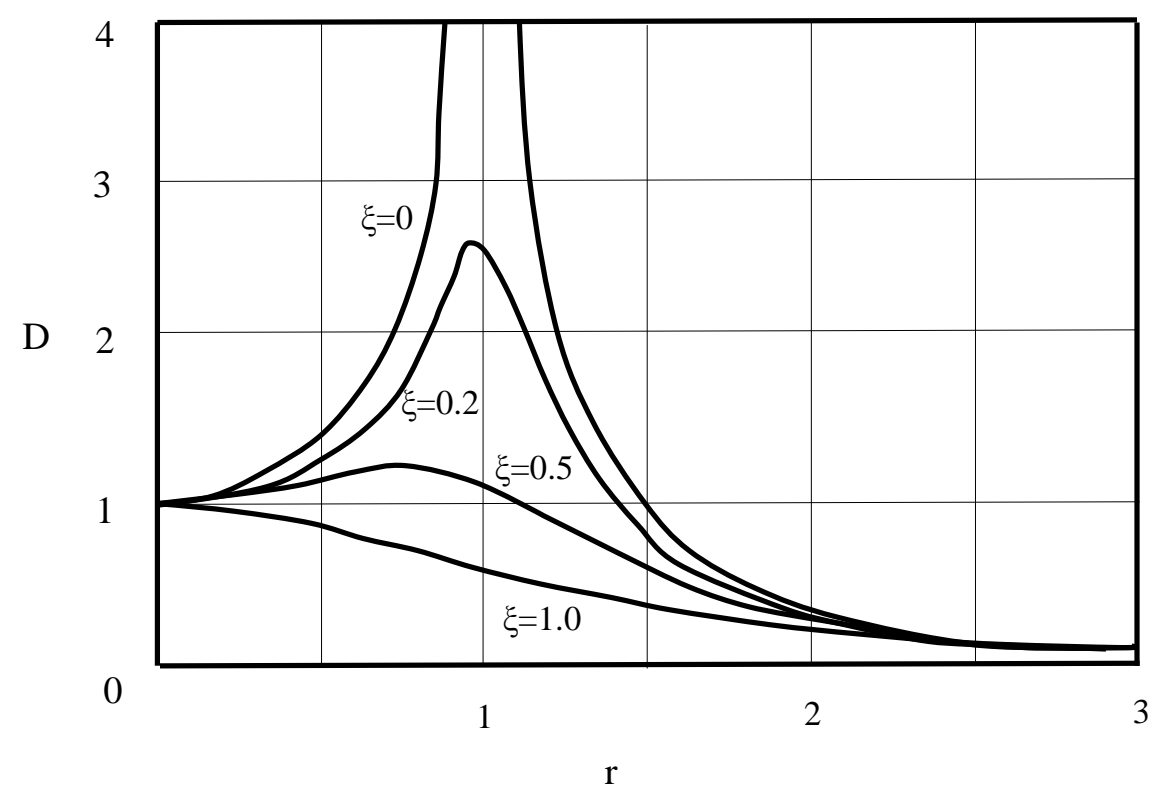

Figura 2-4. Amplificación dinámica en función de la razón de frecuencias para varios valores de amortiguamiento.

Nótese que, en un sistema ligeramente amortiguado, la amplitud máxima ocurre cuando el valor de la razón de frecuencias es muy cercana a $r=1$, o sea la amplificación dinámica tiene su valor máximo en resonancia $(r=1)$.

Introduciendo $r=1$ en la anterior ecuación resulta que en la condición de resonancia la amplificación dinámica es inversamente proporcional a la razón de amortiguamiento, es decir

$$
D(r=1)=\frac{1}{2 \xi}
$$

Aun cuando la amplificación dinámica calculada en la condición de resonancia da un valor cercano a su valor máximo, no es exactamente igual a la respuesta máxima de un sistema con amortiguamiento. Sin embargo, para valores moderados de amortiguamiento, la diferencia entre el valor aproximado dado por la ecuación anterior y el valor máximo exacto es prácticamente insignificante.

\subsubsection{Función de respuesta en frecuencia}

Otra forma de expresar la respuesta de un sistema de un grado de libertad a una excitación armónica es: 


$$
x(t)=X e^{-\xi \omega t} \cos \left(\omega_{D} t-\theta\right)+\frac{F_{0}}{k}\left(\frac{1}{1-r^{2}+2 \xi r i}\right) e^{i \Omega t}
$$

Siendo, como en el caso anterior, el primer sumando de la ecuación la componente transitoria de la respuesta que desaparece con el tiempo y el segundo la respuesta estacionaria presente mientras esté presente la excitación.

Centrándonos de nuevo en este segundo término, se define una función $H(\Omega)$ denominada función compleja de respuesta en frecuencia o función de transferencia [78]:

$$
H(\Omega)=\frac{1 / k}{1-r^{2}+2 \xi r i}
$$

Esta función de transferencia tiene la propiedad de que si sobre el sistema actúa una fuerza que responde a la expresión:

$$
f(t)=F_{0} e^{i \Omega t}
$$

el sistema proporciona una respuesta

$$
x(t)=H(\Omega) F_{0} e^{i \Omega t}
$$

Aún en aquellos casos en que la excitación no es armónica, la respuesta de la estructura puede obtenerse mediante el método de desarrollo en serie de Fourier, como la superposición de respuestas individuales a las componentes armónicas de la excitación externa.

Cualquier excitación $f(t)$ periódica en un intervalo $T$ puede descomponerse en una parte constante y una serie (infinita) de fuerzas armónicas que, al superponerlas, resulta ser la función total fuerza-tiempo dada. Esta descomposición armónica, resulta ser una serie de Fourier de la función excitación, tal como sigue:

$$
f(t)=\frac{a_{0}}{2}+\sum_{j=1}^{\infty} a_{j} \cos (j \Omega t)+b_{j} \operatorname{sen}(j \Omega t)
$$

Además

$$
\begin{array}{ll}
a_{j}=\frac{2}{T} \int_{0}^{T} f(t) \cos (j \Omega t) d t & j=0,1,2, \ldots \\
b_{j}=\frac{2}{T} \int_{0}^{T} f(t) \operatorname{sen}(j \Omega t) d t & j=0,1,2, \ldots
\end{array}
$$


en las cuales $\Omega=2 \pi f$ es la frecuencia fija de la excitación que corresponde al periodo $T$. Las frecuencias de las componentes armónicas son múltiplos de la frecuencia $\Omega$. Una forma alternativa de escribir la componente $j$-ésima de la fuerza es:

$$
f_{j}(t)=a_{j} \cos (j \Omega t)+b_{j} \operatorname{sen}(j \Omega t)=A_{j} \operatorname{sen}\left(j \Omega t-\emptyset_{j}\right)
$$

con una magnitud de fuerza $A_{j}$ y un ángulo de fase $\emptyset_{j}$. Estas variables vienen dadas por:

$$
\begin{aligned}
& A_{j}=\sqrt{a_{j}^{2}+b_{j}^{2}} \\
& \emptyset_{j}=\arctan \frac{b_{j}}{a_{j}}
\end{aligned}
$$

A efectos prácticos, la serie de Fourier se expresa como:

$$
f(t)=\frac{a_{0}}{2}+\sum_{j=1}^{n} A_{j} \cos \left(j \Omega t-\emptyset_{j}\right)
$$

Más utilidad tiene una tercera forma de expresar la serie de Fourier, conocida con el nombre de forma compleja de la serie de Fourier. Basándonos en las relaciones de Euler

$$
\begin{aligned}
& \cos (j \Omega t)=\frac{\left(e^{i j \Omega t}+e^{-i j \Omega t}\right)}{2} \\
& \operatorname{sen}(j \Omega t)=\frac{\left(e^{i j \Omega t}-e^{-i j \Omega t}\right)}{2 i}
\end{aligned}
$$

y haciendo que

$$
c_{j}=\frac{\left(a_{j}-i b_{j}\right)}{2}
$$

obtenemos

$$
f(t)=\sum_{-\infty}^{\infty} c_{j} e^{i j \Omega t}
$$

siendo

$$
c_{j}=\frac{1}{T} \int_{-T / 2}^{T / 2} f(t) e^{-i j \Omega t} \quad j=0, \pm 1, \pm 2, \ldots
$$

Finalmente podemos concluir que siendo la ecuación de equilibrio de un sistema de un grado de libertad:

$$
m \ddot{x}(t)+c \dot{x}(t)+k x(t)=f(t)
$$


Si f(t) es periódica podrá desarrollarse en serie de Fourier, según:

$$
f(t)=\sum_{-\infty}^{\infty} c_{j} e^{i j \Omega t}
$$

y si el sistema es lineal, la respuesta será la suma de las respuestas a cada término de la serie anterior. Siendo cada una de estas respuestas, la respuesta ante una fuerza de carácter armónico, que puede calcularse multiplicando por la correspondiente función de transferencia, se tendrá que:

$$
x(t)=\sum_{-\infty}^{\infty} H(j \Omega) c_{j} e^{i j \Omega t}
$$

Para extender el resultado anterior sobre las series de Fourier al caso de las funciones no periódicas, basta hacer tender a infinito el periodo $T$ de la función $f(t)$. Cuando el periodo $T$ tiende a infinito, la frecuencia fundamental $f$ tiende a cero.

El fundamento teórico de la aplicación del análisis modal estriba en la relación existente entre la matriz de transferencia $H(\Omega)$ y las frecuencias y modos naturales de vibración [77].

\subsubsection{Modos de vibración}

Los modos de vibración se obtienen de expresar los desplazamientos para cada frecuencia natural de la siguiente forma [74]:

$$
\boldsymbol{x}(t)=q(t) \varphi
$$

donde

$q(t) \quad$ son las coordenadas naturales

$\varphi$

son los modos naturales de vibración

Cada modo natural de vibración está asociado a una frecuencia natural y a unos vectores modales y son únicos para un sistema dado, excepto por la magnitud de los vectores modales. La forma modal es única pero la amplitud no. Por ello, es conveniente normalizar dichos vectores, es decir, asignar un valor dado a un componente del vector modal o a la magnitud del vector modal. 
Los modos de vibración (también denominados vectores propios) tienen una propiedad de ortogonalidad que se puede expresar del siguiente modo:

$$
\begin{aligned}
\boldsymbol{\phi}_{i}^{T} \boldsymbol{M} \boldsymbol{\phi}_{j} & = \begin{cases}0 & i \neq j \\
\boldsymbol{m} & i=j\end{cases} \\
\boldsymbol{\phi}^{T}{ }_{i} \boldsymbol{K} \boldsymbol{\phi}_{j} & = \begin{cases}0 & i \neq j \\
\boldsymbol{k} & i=j\end{cases}
\end{aligned}
$$

Donde i,j representan los GDL y $\boldsymbol{m}$ y $\boldsymbol{k}$ se denominan masa modal y rigidez modal respectivamente.

Es habitual normalizar los modos de vibración respecto a la matriz de masa $\boldsymbol{M}$, cumpliéndose en este caso que

$$
\begin{aligned}
& \phi^{T} M \phi=I \\
& \phi^{T} K \phi=\lambda
\end{aligned}
$$

siendo $\boldsymbol{I}$ la matriz identidad y $\lambda$ la matriz de valores propios indicados más adelante.

La relación entre los modos normalizados $\left(\varphi_{\text {nor }}\right)$ y los calculados de manera general $\left(\varphi_{\text {gen }}\right)$ es la siguiente:

$$
\phi_{\text {nor } i}=\frac{\phi_{\text {gen } i}}{\sqrt{m_{i}}}
$$

La principal utilidad de los modos de vibración es que nos permiten desacoplar las ecuaciones de movimiento.

Para el caso de sistemas de N GDL no amortiguados y suponiendo que no actúan cargas exteriores las ecuaciones diferenciales de equilibro se reducen a

$$
M \ddot{x}(t)+\boldsymbol{K} \boldsymbol{x}(t)=\mathbf{0}
$$

donde

$\boldsymbol{x}(t) \quad$ Vector desplazamiento respecto a la posición de equilibrio

M Matriz de masas

K Matriz de rigidez 
La solución a dicho problema es análoga a la de 1 GDL por lo que la ecuación para $\mathrm{N}$ GDL se puede expresar del siguiente modo

$$
\left(-\omega^{2} M+K\right) A=0
$$

donde A es una constante que dependen de las condiciones iniciales del movimiento y $\omega$ es la frecuencia natural sin amortiguamiento.

Si denominamos $\lambda=-\boldsymbol{\omega}^{2}$ y $\boldsymbol{\varphi}=\mathbf{A}$, la ecuación anterior corresponde al problema de valores y vectores propios generalizado:

$$
K \phi=\lambda M \phi
$$

donde $\lambda$ es una matriz diagonal cuyos elementos son los valores propios y $\varphi$ es la matriz cuyas columnas son los vectores propios asociados, normalizados respecto a la matriz $\boldsymbol{M}$ (modos de vibración).

Teniendo en cuenta todo lo anterior, realizamos la transformación de coordenadas reflejada en la ecuación (2-45) en la ecuación (2-51), resultando:

$$
\boldsymbol{M} \ddot{\boldsymbol{q}}(t) \boldsymbol{\phi}+\boldsymbol{K} \dot{\boldsymbol{q}}(t) \boldsymbol{\phi}=\mathbf{0}
$$

Si multiplicamos ambos términos de la ecuación por $\boldsymbol{\varphi}^{T}$, resulta

$$
\boldsymbol{\phi}^{T} \boldsymbol{M} \boldsymbol{\phi} \ddot{\boldsymbol{q}}(t)+\boldsymbol{\phi}^{T} \boldsymbol{K} \boldsymbol{\phi} \dot{\boldsymbol{q}}(t)=\mathbf{0}
$$

o lo que es lo mismo

$$
\boldsymbol{I} \ddot{\boldsymbol{q}}(t)+\boldsymbol{\lambda} \dot{\boldsymbol{q}}(t)=\mathbf{0}
$$

Tratándose de este modo de un sistema de ecuaciones desacopladas en la que no interviene mas que la variable $\boldsymbol{q}(t)$.

Para el caso más general (vibraciones en sistemas amortiguados) para N GDL la ecuación que gobierna el movimiento es la ecuación (2-2), que recordamos a continuación

$$
\boldsymbol{M} \ddot{\boldsymbol{x}}(t)+\boldsymbol{C} \dot{\boldsymbol{x}}(t)+\boldsymbol{K} \boldsymbol{x}(t)=\boldsymbol{f}(t)
$$

En este caso para realizar la transformación de coordenadas reflejada en la ecuación (245) es necesario diagonalizar las matrices $\boldsymbol{K}, \boldsymbol{M}$ y $\boldsymbol{C}$, lo cual no es posible en el caso de la matriz de amortiguamiento si no realizamos hipótesis simplificativas. A la hipótesis de 
modelización del amortiguamiento que permite diagonalizar la matriz $\boldsymbol{C}$ al mismo tiempo que las matrices $\boldsymbol{K}$ y $\boldsymbol{M}$ (y por lo tanto desacoplar las ecuaciones del movimiento) se le denomina amortiguamiento proporcional. Cuando no es posible diagonalizar la matriz de amortiguamiento se dice que se está en el caso de amortiguamiento no proporcional.

Suponiendo que la matriz $\boldsymbol{C}$ es diagonizable y aplicando la consiguiente transformación de coordenadas se llega a un sistema de $\mathrm{N}$ ecuaciones diferenciales (desacopladas) de la forma:

$$
\ddot{\boldsymbol{q}}(t)+2 \xi \boldsymbol{\omega} \dot{\boldsymbol{q}}(t)+\boldsymbol{\omega}^{2} \boldsymbol{q}(t)=\boldsymbol{g}(t)
$$

Donde $g(\mathrm{t})=\boldsymbol{\varphi} f(\mathrm{t}) / \boldsymbol{m}$. 


\subsection{Análisis modal experimental}

El análisis modal experimental trata, a través de la respuesta del sistema, de inferir las ecuaciones del mismo y por tanto las características físicas que lo determinan dinámicamente, los parámetros modales.

El objetivo del análisis modal experimental involucra las tareas y trabajos necesarios para obtener las medidas directas de la respuesta de un sistema y, a partir de ellas, obtener los parámetros modales que caracterizan el sistema dinámico. Los parámetros modales, se pueden estimar a través de una serie de medidas, almacenadas de forma discreta, en los diferentes dominios (tiempo y/o frecuencia). Estas medidas se pueden procesar una a una, o bien, en grupos parciales o completos de forma simultánea. Hay un gran abanico de posibilidades a la hora de adquirir todas estas medidas.

Aunque existen diferentes clasificaciones, se distinguen generalmente tres tipos de análisis modal experimental [79], análisis modal experimental tradicional (EMA), análisis modal operacional (OMA) y análisis modal operacional con entradas exógenas (OMAX).

En el EMA, los parámetros modales se obtienen de las FRFs que relacionan la salida (respuesta) con la entrada (excitación). Para este tipo de análisis es necesario conocer la entrada y la salida del sistema.

El OMA se basa en la medida de la respuesta de la estructura usando sólo la excitación ambiental y las fuerzas de servicio que actúan sobre la misma. Se usa para obtener una descripción modal de la estructura bajo sus condiciones de operación. En vez de cargar la estructura artificialmente y tratar la excitación ambiental como una fuente de ruido no deseado, la excitación natural se usa como fuente de excitación.

La ventaja de emplear una excitación artificial es que hay muchos más procedimientos para identificar los parámetros y características de los sistemas, con mayor precisión en la identificación de ciertos parámetros como los coeficientes de amortiguamiento.

En el caso del análisis de la respuesta a las excitaciones ambientales no se puede obtener la misma información que en el caso de que excitemos el sistema artificialmente, ya que las masas modales no se pueden obtener sin el conocimiento de las fuerzas de entrada. 
Por otra parte, hay sistemas que son difíciles de excitar artificialmente debido a su tamaño, forma o localización. También hay casos en los que surgen problemas debido a no linealidades introducidas por un nivel de respuesta muy elevado por emplear métodos artificiales de excitaciones.

Las principales ventajas de este tipo de ensayos (OMA) son que requieren un menor tiempo para su ejecución ya que sólo es necesario colocar los equipos de medida; son más baratos puesto que no necesitan la adquisición de equipos excitadores y puede realizarse in situ; no interfieren ni interrumpen el funcionamiento normal de la estructura; la respuesta medida es representativa de las condiciones reales de funcionamiento del sistema (p. ej. Estructura); se evita la aplicación de cargas artificiales que conllevan un riesgo de dañar la estructura. Sin embargo, el uso de este tipo de ensayos produce respuestas pequeñas $\mathrm{y}$, a menudo, mezcladas con ruido y como la excitación es desconocida el análisis es más difícil por lo que se necesitan equipos más sensibles siendo necesario un análisis de datos cuidadoso.

Cuando un ensayo se lleva a cabo en condiciones de operación y además se aplican una o varias fuerzas artificiales, éste se denomina OMAX.

\subsubsection{Técnicas de excitación}

Hay muchas formas posibles de excitar una estructura. Se puede utilizar como excitación la producida por el ambiente o se puede producir intencionadamente.

Las técnicas de excitación en este segundo caso se pueden clasificar en dos tipos: las que permanecen en contacto con la estructura durante el ensayo y las que no [72].

Las primeras pueden proporcionar una excitación continua (sinusoidal, aleatoria, etc) o transitoria (pulso), y para ello se emplean los excitadores electromecánicos, electromagnéticos y electrohidráulicos.

Los excitadores electromecánicos se dividen en dos tipos según el tipo de desplazamiento de la masa que se mueve: los hay de movimiento rectilíneo y rotatorio. Los primeros están formados por dos elementos: una base que no se desplaza y una masa que sí y que es la que produce la vibración. Hay dos maneras de emplear este tipo de excitadores. La 
primera cosiste en fijar la base a la estructura que se desea excitar y mover la otra masa. La otra opción es fijar la masa en movimiento a la estructura y hacerla oscilar.

El excitador electromagnético es el más empleado en los ensayos dinámicos debido al amplio rango de fuerzas que puede realizar y de frecuencias que puede excitar. Está formado por dos partes: la base del excitador, que produce el campo magnético, y la parte móvil o tabla, que es la que se une a la estructura que se está ensayando. La fuerza que mide el transductor es la fuerza que se genera en el excitador. Esta fuerza es ligeramente diferente de la que se transmite a la estructura, ya que parte de esta fuerza se invierte en vencer la inercia de la tabla.

El excitador electrohidráulico se usa generalmente para producir excitaciones de baja frecuencia que requieren una gran cantidad de fuerza y bajas velocidades. Consiste en un pistón que transmite la carga a la estructura y que se mueve en un cilindro. El pistón se encuentra sometido a una presión de aceite muy elevada bajo control de una servoválvula que es eléctricamente alimentada. La presión es aplicada a un lado y luego al otro del pistón principal, haciendo que se mueva hacia delante y hacia detrás.

Ejemplos de técnicas en las que la excitación aplicada está sólo en contacto durante un periodo de tiempo corto, son el impacto y la excitación estática (aplicar una carga estática a una estructura y retirarla repentinamente).

En el excitador de martillo (martillo de impacto) se pueden diferenciar las siguientes partes: la masa sísmica $\mathrm{m}_{1}$ que incluye la masa de la punta del martillo, la masa $\mathrm{m}_{2}$ que representa la masa efectiva del martillo, y un amortiguador y un resorte que modelan la célula de carga. La fuerza medida es la producida por la masa colocada detrás del transductor, multiplicada por la aceleración. Pero la fuerza con la que realmente se está excitando a la estructura es igual a la masa total del martillo (incluyendo tanto la masa de la punta como la del transductor de fuerza) multiplicada por la aceleración durante el impacto. Por tanto, la verdadera fuerza que actúa se obtiene de multiplicar la fuerza medida por la relación entre la masa total y la masa colocada detrás del transductor. Los martillos se pueden fabricar con pesos que varían entre unos pocos gramos a varias toneladas, cubriendo el rango de $0-5000 \mathrm{~Hz}$ con los más pequeños y de $0-10 \mathrm{kHz}$ con los más grandes. 


\subsubsection{Medida de la excitación y la vibración}

Los ensayos de vibraciones requieren el uso de transductores para medir tanto el movimiento como las fuerzas. Un transductor se puede definir como un componente del sistema de medida capaz de procesar información y energía [72].

Los transductores de fuerza pueden estar basados en la aplicación de las leyes de la estática, en el fenómeno de la reacción elástica o en el fenómeno de la piezoelectricidad.

A continuación pasamos a describir dos de los tipos más frecuentes de transductores de fuerza, la celda de carga y el transductor piezoeléctrico.

- El transductor de peso o celda de carga está dotado de extensómetros o "straingage" (dispositivos que relacionan su deformación porcentual con una variación de resistencia). Esta celda transforma una fuerza aplicada en una variación de la tensión presente en la salida de un puente de extensómetros. Generalmente en la celda de carga hay varios extensómetros los cuales están montados en un elemento mecánico alterable y conectados en puente. El funcionamiento consiste en que al aplicar sobre este dispositivo una fuerza se obtiene una deformación de la estructura metálica alterable.

- Los dispositivos piezoeléctricos se usan especialmente cuando las fuerzas a medir son dinámicas. Aprovechan el fenómeno en el que variaciones de carga se verifican en algunos materiales cuando éstos están expuestos a fuertes acciones físicas. Se usan en instrumentos utilizados para el estudio de las vibraciones.

Los transductores de vibración son empleados para medir la velocidad, desplazamiento y/o aceleración de los sistemas sometidos a vibración. En general, los transductores empleados en el análisis de vibración convierten la energía mecánica en energía eléctrica, lo que significa que producen una señal eléctrica que es función de la vibración.

Si bien el acelerómetro es el transductor para la medida de vibraciones más empleado debido a su pequeño tamaño, su amplio rango de sensibilidad y su gran rango de frecuencias útiles, a continuación se muestran diferentes tipos de transductor usados para la medición de vibración:

- Transductores de aceleración 
○ Acelerómetros piezorresistivos

○ Acelerómetros piezoeléctricos

- Transductores de desplazamiento

- Transformador diferencial de variación lineal (LVDT -Linear Variable Differential Transformer)

○ Corriente Eddy

- Capacitivos

- Transductores de velocidad

○ Vibrómetros Doppler Láser

\subsubsection{Análisis y procesado de las señales}

Las señales analógicas de entrada se filtran, se muestrean y se digitalizan para obtener una serie de registros digitales. La relación de muestreo y la longitud de los registros determinan el rango de frecuencias y la resolución del análisis.

Cuando la señal de respuesta ha sido acondicionada se conduce a un analizador para su procesado. Hay dos tipos de analizadores: analógicos y digitales. Los analógicos prácticamente están en desuso puesto que los digitales tienen mayores rendimientos y versatilidad, además cada vez se usan más los sistemas de adquisición de datos por medio de ordenadores.

El análisis de los datos se puede realizar en el dominio del tiempo (la señal se expresa con respecto al tiempo) y en el dominio en frecuencia (la señal se expresa con respecto a la frecuencia). A la representación de la señal en el dominio de la frecuencia se le denomina espectro de frecuencia. Una de las herramientas matemáticas más importantes para llevar a cabo la transformación de señales del dominio del tiempo al dominio de la frecuencia, y viceversa, es la transformada de Fourier (discreta (DFT - Discrete Fourier Transform), rápida (FFT - Fast Fourier Transform)...). Hay dos formas de realizar el análisis en frecuencia, una es mediante el analizador FFT y otra es mediante el filtrado digital.

Existen una serie de efectos que distorsionan las señales debidos a la conversión de la señal analógica a digital y/o a la transformación de las mismas del dominio del tiempo al de la frecuencia, siendo los más importantes los que se conocen con los términos ingleses de aliasing, leakage y picket-hence [72]: 
- Aliasing: este fenómeno consiste en que las frecuencias superiores a la mitad de la frecuencia de muestreo aparecen como frecuencias distintas en la señal muestreada. Para poder detectar una frecuencia determinada $\mathrm{f}$ es necesario muestrear de forma que se tengan al menos dos puntos por ciclo, es decir, la frecuencia de muestreo, $f s$, debe ser mayor que $2 f$. Este fenómeno se soluciona empleando un filtro anti-aliasing y eligiendo una frecuencia de muestreo adecuada. El filtro anti-aliasing lo que hace es eliminar las componentes de alta frecuencia de las señales analógicas medidas.

- Leakage: este efecto se debe al hecho de que al medir la vibración no se obtiene la variación total de la variable con el tiempo ya que, en teoría, ésta dura un tiempo infinito. en su lugar se mide una porción temporal de la misma, es decir, se realiza un registro temporal finito. Se puede evitar mediante el empleo de funciones ventana.

- Picket-Hence: este fenómeno se hace evidente cuando la señal que está siendo analizada no está sintonizada con alguna de las frecuencias centrales de los filtros. En este caso, las componentes de frecuencias afectadas aparecerán con amplitudes inferiores a la real. Se soluciona mediante el empleo de la ventana apropiada y aumentando la resolución en frecuencia.

También hay que tener en cuenta el ruido, que es una señal aleatoria, generalmente de alta frecuencia pero con un ancho de banda también muy amplio, que se superpone a la señal que se está midiendo. Este ruido distorsiona la señal, y si la relación ruido-señal es muy alta, puede falsear totalmente los datos. El ruido puede ser debido a la red eléctrica, a movimientos de los cables y de las conexiones, a interferencias electromagnéticas producidas por los cables o al equipo que se está usando. Se puede evitar usando filtros.

El filtro es un dispositivo para la descomposición de las oscilaciones en base a sus frecuencias componentes [80]. Este introduce una atenuación relativamente baja para las oscilaciones contenidas en una o más bandas de frecuencias e introduce una atenuación relativamente alta sobre las oscilaciones contenidas en otras bandas de frecuencia. Los filtros se emplean para atenuar partes no deseadas de las señales en el dominio de la frecuencia. Aunque el funcionamiento de los filtros en realidad es más complicado y hay más aspectos a tener en cuenta se puede decir que un filtro tiene la propiedad de eliminar 
ciertas frecuencias de una señal y dejar pasar las demás sin alterarlas. Existen varios tipos de filtros según las frecuencias que deja pasar [72]:

- El filtro paso-bajo elimina las frecuencias por encima de cierto valor. Un ejemplo es el filtro anti-aliasing.

- El filtro paso-alto elimina las frecuencias por debajo de la frecuencia de corte.

- El filtro paso-banda deja pasar sólo una banda definida por dos frecuencias. Permitirá pasar todas las componentes de la señal a lo largo de su banda de frecuencias permitidas, y atenuará completamente todas las demás componentes a otras frecuencias. Puede ser de banda constante o variable.

- El filtro rechazo de banda elimina las frecuencias que se encuentran entre dos valores.

Los filtros se caracterizan en el dominio de frecuencia por cuatro parámetros: frecuencia central (valor medio geométrico o aritmético de los límites de frecuencia), ancho de banda (da información sobre su habilidad para separar componentes de amplitudes similares y por tanto determina la resolución del análisis), rizado (caracteriza la incertidumbre con la que la señal puede ser determinada) y selectividad (indica la capacidad que tiene para separar componentes de frecuencias próximas pero con niveles diferentes).

Otras herramientas en el análisis y procesamiento de las señales son las ventanas, funciones matemáticas que nos permiten aislar una porción de señal a analizar y desechar el resto en mayor o menor medida. Hay diferentes tipos de ventanas según la aplicación que se vaya a desarrollar (Rectangular, Hanning, Transitoria, etc). Cada una de las ventanas enfatizará sobre las partes de la señal de cierta manera, obteniéndose así diferentes espectros como resultado. Las ventanas se emplean para que las señales muestreadas cumplan mejor los requisitos de periodicidad de la Transformada de Fourier, además de minimizar la potencial distorsión que pueden producir los efectos del leakage.

A continuación se indican los principales usos de cada tipo de ventana [72]:

- Ventana rectangular o uniforme: se caracteriza porque no realiza ningún tipo de ponderación en el tiempo que dura el registro. Se emplea para analizar transitorios de duración menor que el tiempo de registro. En general, sólo se debe emplear en aquellos casos en los que no sea necesario realizar un filtrado para eliminar el leakage. 
- Ventana Hanning: se caracteriza porque realiza una ponderación que da menor importancia a los datos tomados al principio y al final del muestreo. Es una ventana de uso general y fácil de implementar. En general, esta ventana es recomendable para los análisis de señales periódicas. Es la mejor ventana para realizar análisis de sistemas en los que la excitación sea una señal aleatoria.

- Ventana Kaiser-Bessel: se debe emplear para señales periódicas que requieren una selectividad en frecuencia buena. No debe usarse para señales transitorias.

- Flat Top: esta ventana se ha diseñado para propósitos de calibración principalmente. No se recomienda su uso para señales aleatorias ni transitorias.

- Transitoria: es la que se debe emplear para la medida de transitorios de señales, como puede ser el caso de impactos. Esta ventana toma las medidas sin ponderar mientras dura el contacto y cae a cero al terminar el registro.

- Exponencial: el uso principal de esta ventana es en el análisis de transitorios de mayor duración que el registro medido. Las señales que presentan una disminución exponencial de la amplitud se deben ponderar con esta ventana. Este es el caso habitual de la respuesta de estructuras poco amortiguadas cuando se excitan mediante un impacto. 


\subsection{Técnicas de análisis modal aplicadas a la Pasarela del Museo de la Ciencia}

La Pasarela del Museo de la Ciencia es una estructura en servicio situada en Valladolid sobre el río Pisuerga. Fue inaugurada en 2004 y diseñada por los arquitectos Rafael Moneo y Enrique de Teresa. Tiene una longitud total de 234 metros repartidos en cuatro vanos: tres formados por barras metálicas de 51, 111 y 21 metros de luz (vano 2, 3 y 4 respectivamente), y uno de hormigón de 45 metros (vano 1). Éste último es muy rígido por lo que no se considera en el estudio objeto del presente trabajo.

El vano principal cuenta con un pretensado longitudinal y transversal lo que le hace ser bastante rígido y mejora su comportamiento dinámico frente a las vibraciones producidas por los humanos. Sin embargo, el vano 2 , de $51 \mathrm{~m}$, no dispone de ningún sistema de pretensado de forma que es susceptible a vibraciones producidas por humanos.

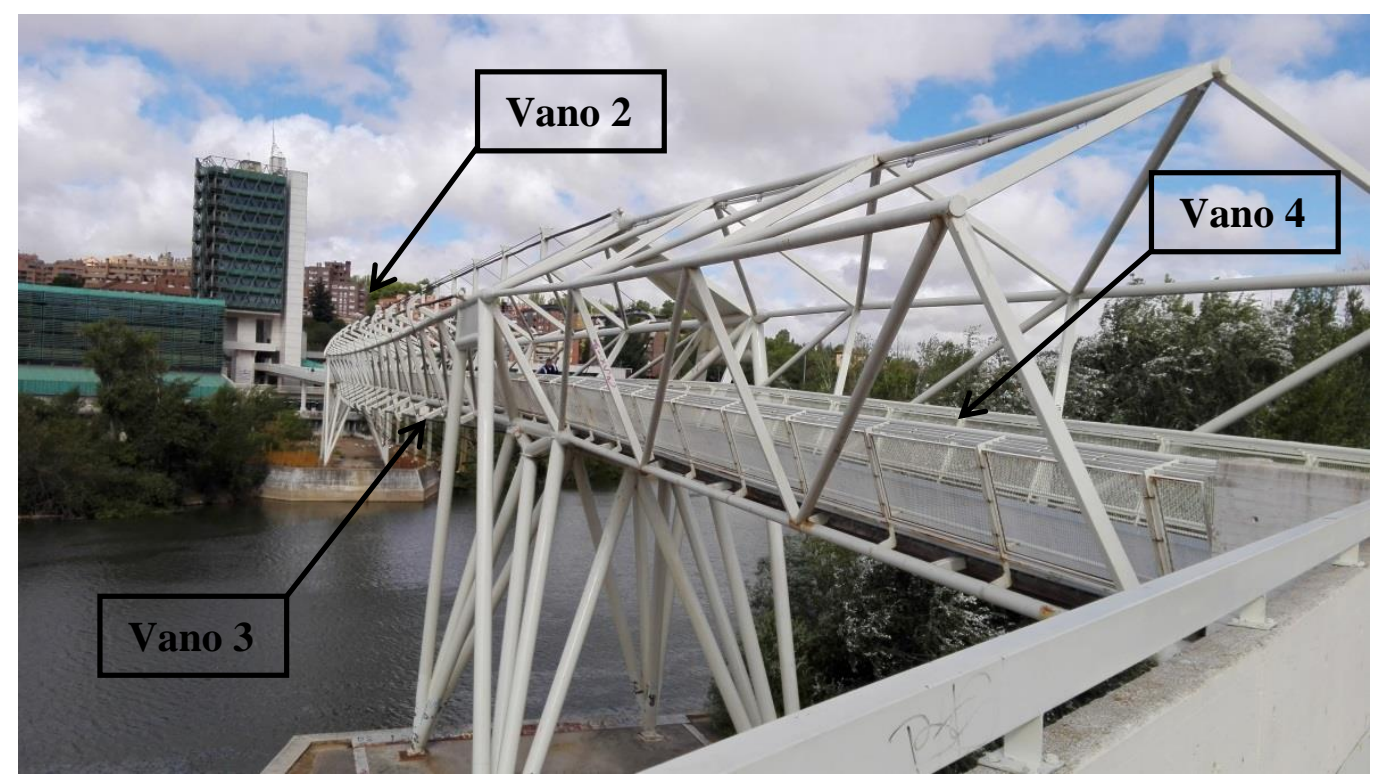

Figura 2-5. Pasarela del Museo de la Ciencia (Valladolid). Vista general. 


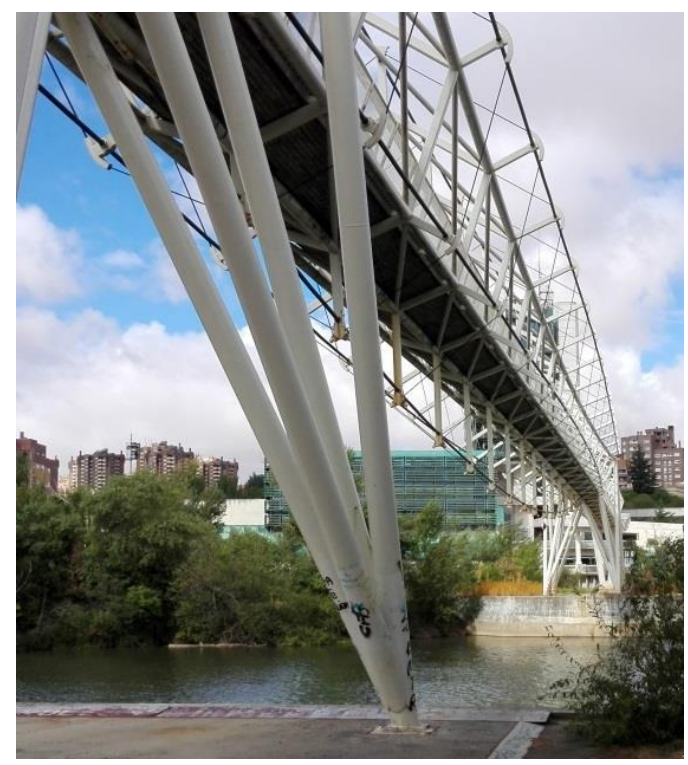

Figura 2-6. Pasarela del Museo de la Ciencia (Valladolid). Vista del vano principal.

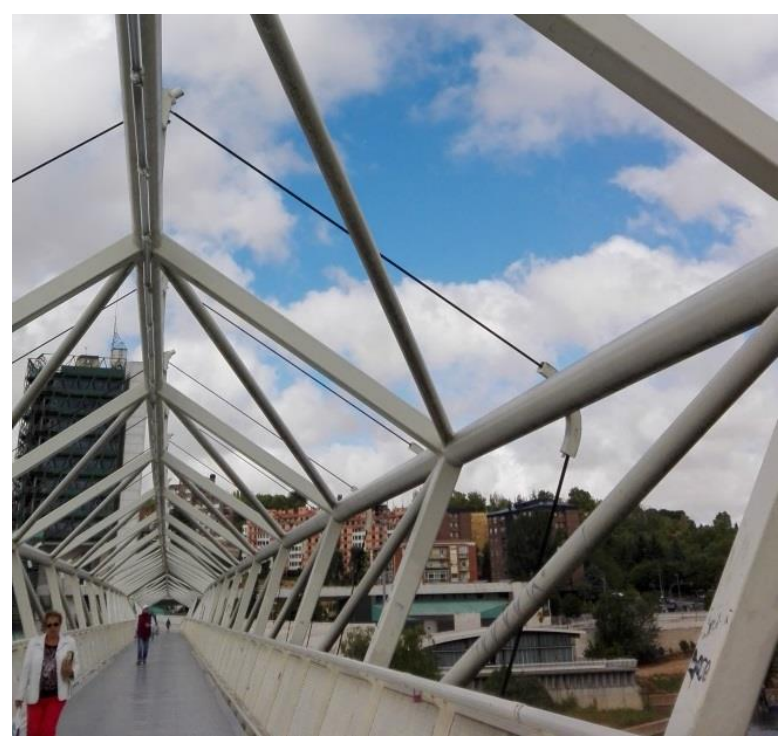

Figura 2-7. Pasarela del Museo de la Ciencia (Valladolid). Detalle del pretensado perimetral del vano principal. 


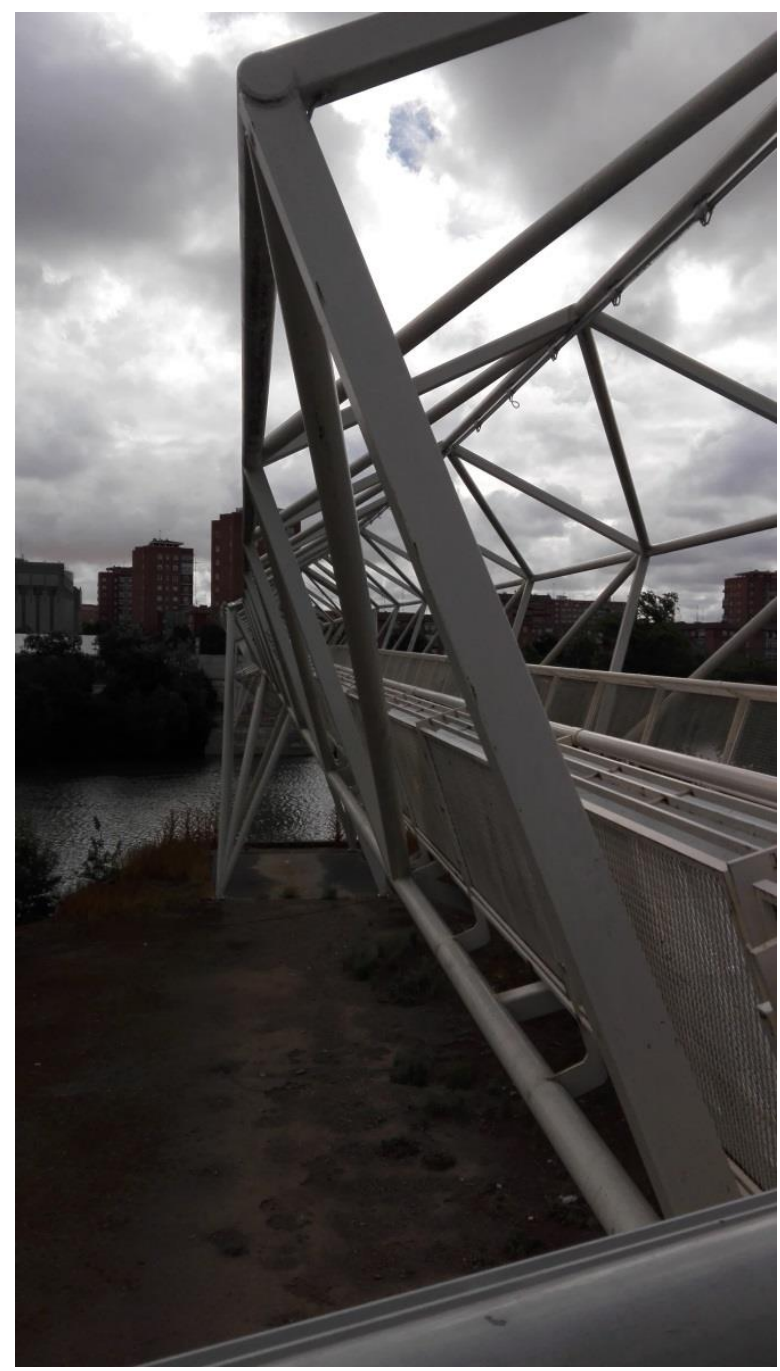

Figura 2-8. Pasarela del Museo de la Ciencia (Valladolid). Vista del vano 2.

Según [2] para la caracterización dinámica de la pasarela inicialmente se efectuó una monitorización durante varios meses de las aceleraciones verticales y laterales en el centro de cada uno de los tres vanos metálicos de la pasarela observándose que los niveles de vibración en la dirección lateral eran considerablemente menores que en la dirección vertical y que el nivel de vibraciones del vano 2 era notablemente mayor que el del vano 3. También se realizó un análisis espectral preliminar de los registros de aceleración en dirección vertical y lateral para identificar las posibles frecuencias naturales.

Posteriormente se realizó un OMA de la pasarela completa, registrándose mediciones de la aceleración vertical en 75 puntos distribuidos a lo largo de los tres vanos metálicos de la pasarela utilizando una frecuencia de muestreo de $100 \mathrm{~Hz}$. Se obtuvieron registros de ocho acelerómetros de 10 minutos de duración cada uno, empleando en cada vano un acelerómetro de referencia (tres en total). Se emplearon tres tipos de acelerómetros: MMF 
KS48C (6 unidades), PCB 393B04 (1 unidad) y PCB 393C (1 unidad). Todos ellos del tipo sísmico (piezoeléctrico) con sensibilidad $1000 \mathrm{mV} / \mathrm{g}$ y con un rango de frecuencias entre $0.1 \mathrm{~Hz}$ hasta $2000-4000 \mathrm{~Hz}$.

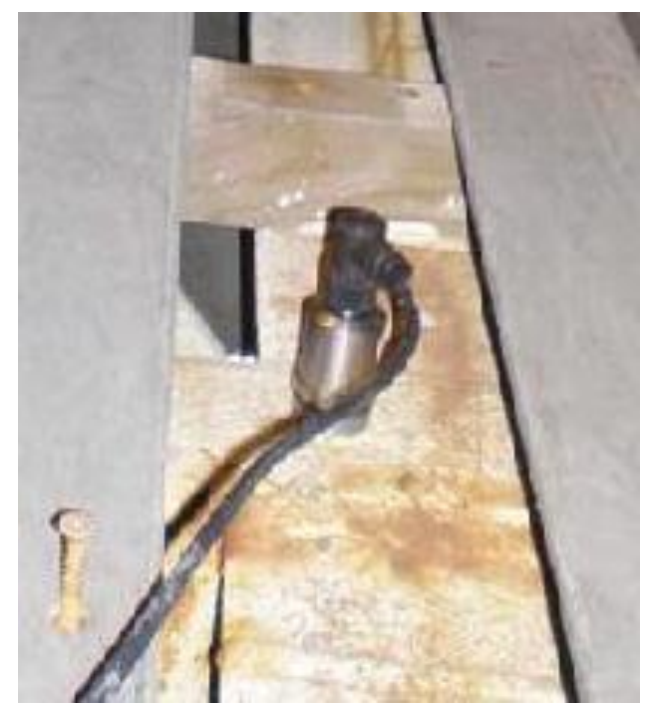

Figura 2-9. Acelerómetro empleado en el OMA.

El equipo de medida fue un HBM MGCplus con el software Catman Easy.

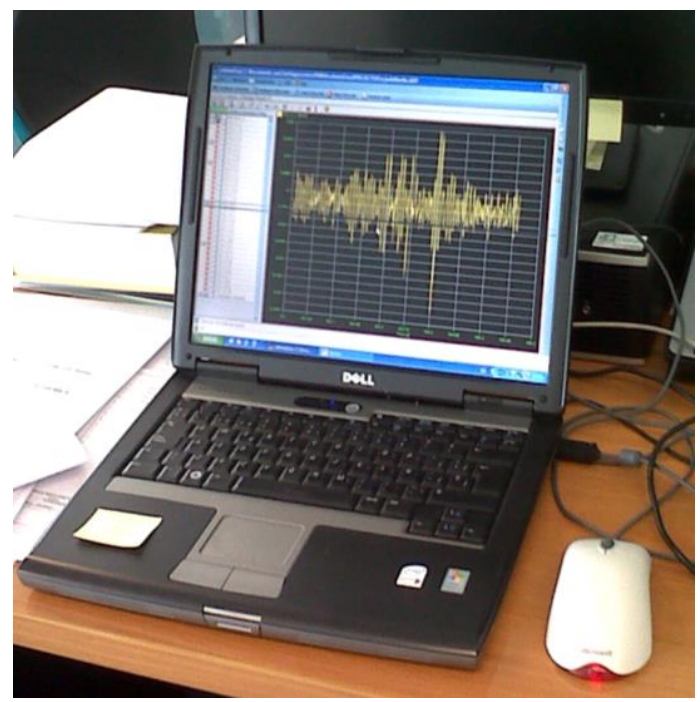

Figura 2-10. Equipo de medida utilizado en el OMA.

Teniendo en cuenta que las mayores aceleraciones en la pasarela se alcanzaban en el vano 2 de la pasarela y en vertical se realizó un EMA de dicho vano, generándose la fuerza de excitación con un excitador electrodinámico (electromagnético) de APS Dynamics modelo 400 en modo inercial colocado en el centro del vano [2]. 


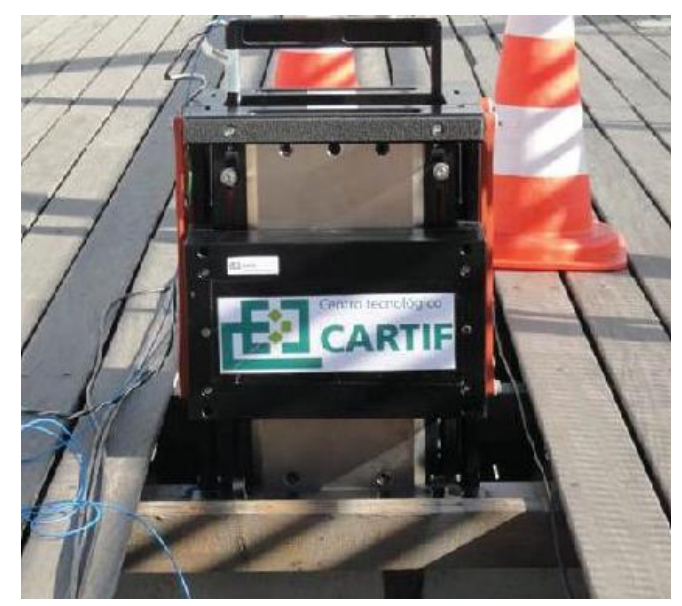

Figura 2-11. Excitador electrodinámico utilizado en el EMA.

La fuerza inducida por el excitador se calculó midiendo la aceleración de la masa inercial y multiplicándola por su magnitud $(30.4 \mathrm{~kg})$. Se utilizaron acelerómetros iguales a los empleados en el OMA, colocados en la línea central de la pasarela. Se utilizó un rango de frecuencias de 0 a $25 \mathrm{~Hz}$ con una resolución de la FFT de 1601 puntos, una ventana de ponderación rectangular y 4 medidas con solapamiento del 50\% [2]. 



\section{CAPÍTULO 3. MODELO MECÁNICO AJUSTADO}


El concepto de método de los elementos finitos se basa en la discretización espacial y temporal de los sistemas estudiados y la aproximación numérica para encontrar soluciones a los problemas de ingeniería y el empleo de estas técnicas es conocido desde hace muchos siglos. Por ejemplo, los antiguos egipcios $(3.000-30$ a. C. aproximadamente) utilizaban métodos de discretizado para determinar el volumen de las pirámides que construían, Arquímedes (287-212 a. C.) utilizaba este método para hallar el volumen de los sólidos o áreas de superficies o el matemático Lui Hui (300 d. C) empleaba un polígono regular de 3072 lados para calcular la longitud de las circunferencias logrando una aproximación al número $\pi$ de 3,1416.

El origen del moderno método de los elementos finitos se remonta a principios del siglo XX cuando algunos investigadores, como Hrennikoff y McHenry, aproximaron y modelizaron un sólido elástico con sección transversal continua usando un conjunto discreto de barras elásticas equivalente [12-13]. Sin embargo, se considera que Courant fue la primera persona en desarrollar un método de elementos finitos en 1943. En una publicación a principios de los cuarenta, Courant propone la utilización de funciones polinómicas para la formulación de problemas elásticos en subregiones triangulares, como un método especial del método variacional de Rayleigh-Ritz para aproximar soluciones [14].

El siguiente paso significativo, en el campo de la ingeniería estructural, fue dado por Levy en 1953 [15] que desarrolló el método de rigidez y desplazamiento, sin embargo sus ecuaciones requerían demasiado trabajo para su solución a mano por lo que este método de análisis numérico no se popularizó hasta el desarrollo de las computadoras.

Entre 1954 y 1955, Argyris y Kelsey [16] emplearon métodos energéticos para el desarrollo del análisis estructural por medio de matrices, lo que marcó una tendencia en la evolución del análisis de elementos finitos. En sus artículos se presenta completamente desarrollada la formulación matricial de la teoría de estructuras y donde están claramente perfilados los métodos de las fuerzas y los desplazamientos.

La que se considera la primera aplicación del método de elementos finitos fue un trabajo realizado por Clough en la Boeing Airplane Company dentro de la unidad de dinámica estructural, dirigido por Turner, para la evaluación de la rigidez del ala delta de un avión. 
Puesto que los modelos de barras no daban buenos resultados, Turner sugirió la división de la superficie en porciones triangulares cuya rigidez fue evaluada utilizando el teorema de Castigliano suponiendo estados constantes de tensión normal y cortante en los triángulos y obteniendo la rigidez total sumando adecuadamente las de cada triángulo (método directo de la rigidez). Se presentó una comunicación sobre este trabajo en la reunión del Institute of Aeronautical Sciences en enero de 1954 en Nueva York, firmada por Turner, Clough, Martin y Topp con el título "Stiffness and deflection analysis of complex structures" (aunque se publicó en 1956). Sin embargo no fue hasta los 60 cuando Clough hizo popular el término "elemento finito", con la primera comunicación en la que se encuentra dicho término publicada en la American Society of Civil Engineers (ASCE) con el título "The Finite Element method in plane stress analysis" [17].

A partir de los años 60, se producen numerosos avances que permitieron el rápido desarrollo de este método como estudios relativos a la matriz de masa, grandes desplazamientos, problemas no lineales, o la aplicación a problemas de tres dimensiones, pandeo, viscoelasticidad, ... [18-27]. Asimismo se produce un gran interés entre los matemáticos por la solución de ecuaciones diferenciales lineales y no lineales mediante el FEM lo que permitió que se empezara a aplicar en otras áreas de la ingeniería, como la transferencia de calor o problemas de flujo de fluidos. [28-33]. Zienkiewicz and Cheung escribieron el primer libro dedicado por completo al FEM en 1967 [34] en el que se presenta una interpretación amplia de este método y su aplicación a diferentes campos.

A mediados de los setenta, Belytschko [35, 36] mejoró las técnicas numéricas aplicables a la resolución de los sistemas de ecuaciones de problemas dinámicos no lineales con grandes desplazamientos.

Es durante esta década cuando se considera que el FEM alcanza la madurez, apareciendo los grandes programas comerciales. En 1971, el software ANSYS se lanza al mercado por primera vez.

Desde entonces hasta nuestros días se han generado una gran cantidad de artículos y publicaciones que demuestran que el FEM es una de las herramientas más potentes y probadas para la solución de problemas de ingeniería y que amplía su campo de aplicación a medida que se realizan más estudios validando dicho método, como sería el caso de la dinámica de estructuras civiles. 
Por otra parte, el desarrollo de diversas técnicas de medición de vibraciones muy fiables ha permitido que las propiedades modales (frecuencias, amortiguamientos, modos de vibración...) sean obtenidas también de forma experimental, lo que ha permitido observar una discrepancia entre las predicciones de los modelos de elementos finitos y los resultados experimentales. Como respuesta a dicho problema se han desarrollado varias líneas de investigación cuyo objetivo ha sido aprovechar las ventajas de los métodos de elementos finitos y de las técnicas experimentales de forma simultánea y cuyo resultado ha sido el ajuste o actualización de modelos de elementos finitos, más conocido como FEM Updating.

A partir de la década de 1980, el ajuste de modelos numéricos ha tenido un crecimiento vertiginoso. Este hecho se debe principalmente al desarrollo de sofisticados instrumentos de medición de vibraciones y veloces analizadores de señales dinámicas de bajo coste. De forma paralela un importante número de investigadores han desarrollado técnicas experimentales y analíticas que permiten procesar y utilizar eficientemente los datos dinámicos.

Sin embargo, la extensa investigación que se ha realizado en las últimas décadas sobre el FEM Updating nos indica que aún no ha alcanzado su madurez y que no existe ningún procedimiento general ni completamente fiable que pueda ser formulado para todos los casos. Algunos autores [37-43, 81] han publicado y comparado varias técnicas pero no se alcanza ningún acuerdo sobre métodos o prácticas. En la mayoría de los casos la solución parece depender de los parámetros y constantes seleccionados así como de la técnica empleada. 


\subsection{El método de elementos finitos}

El FEM permite realizar un modelo matemático de cálculo de un sistema real, especialmente en el campo ingenieril, cuya complejidad dificulta su resolución por métodos exactos de análisis. El Finite Element Analysis (FEA) es una poderosa técnica computacional para obtener soluciones aproximadas a una gran variedad de problemas de ingeniería. Este tipo de análisis ha llegado a ser un paso esencial en el diseño o modelización de fenómenos físicos en varias disciplinas de ingeniería.

Un fenómeno físico suele producirse en un medio continuo (sólido, líquido o gas) involucrando varias variables de campo. Dichas variables de campo varían de un punto a otro, por lo que es necesario procesar un número infinito de soluciones en el dominio (medio continuo con condiciones de contorno conocidas).

La base del FEA reside en la descomposición del dominio en un número finito de subdominios (denominados elementos) y expresando cada variable de campo desconocida en términos de una función de aproximación ficticia para cada elemento. Estas funciones (también denominadas funciones de interpolación) se definen en función de valores de las variables de campo en determinados puntos, denominados nodos. Dichos nodos generalmente se localizan a lo largo de los contornos del elemento y conectan elementos adyacentes.

La capacidad de discretizar los dominios irregulares en elementos finitos hace de este método una valiosa y práctica herramienta de análisis para la solución de los problemas de contorno, iniciales y de valores propios derivados de varias disciplinas de ingeniería [82].

La transformación de los problemas de ingeniería en una representación matemática se logra discretizando el dominio en elementos que están conectados entre sí mediante los nodos comunes. Un nodo especifica la localización espacial donde se encuentran los grados de libertad (variables nodales asignadas a un elemento) y las acciones del problema físico. Los nodos comunes proporcionan continuidad a las variables nodales (grados de libertad) que dependen de la naturaleza física del problema y del tipo de elemento. 
Dependiendo de la geometría y de la naturaleza del problema, el dominio puede ser discretizado empleando elementos tipo línea, área o volumen. Cada elemento se define por una secuencia específica de números de nodos globales.

Los pasos básicos involucrados en el análisis mediante elementos finitos pueden resumirse en [69]:

- Fase de Pre-procesado:

○ Discretización del dominio en elementos finitos, es decir, subdividir el problema en nodos y elementos.

- Definir una función de forma que represente el comportamiento físico de cada elemento, es decir, una función aproximada continua que se supone represente la solución del problema.

- Desarrollar las ecuaciones de cada elemento.

- Ensamblar todos los elementos que definen el dominio para obtener la matriz de rigidez global.

- Aplicar las condiciones iniciales y de contorno así como las cargas.

- Fase de Resolución

- Resolver una serie de ecuaciones algebraicas (lineales y no lineales) simultáneamente a la obtención de los resultados nodales, como desplazamientos, velocidades y aceleraciones (problemas estáticos, dinámicos...), temperatura (problemas de transferencia de calor...)

- Fase de Post-procesado

- Obtener otro tipo de información importante como por ejemplo las frecuencias naturales, los modos de vibración, tensiones, etc. 


\subsection{FEM Updating}

A través del FEM Updating se trata de generar modelos fiables a través de ajustes paramétricos en los mismos, de forma que se minimicen las diferencias entre las predicciones analíticas y los resultados experimentales.

Los programas informáticos disponibles para la formulación de modelos matemáticos computacionales son cada vez más poderosos debido al constante desarrollo de algoritmos eficientes y a la reducción en las restricciones impuestas por el hardware, lo que ha constituido una de las principales herramientas del análisis moderno de problemas de ingeniería haciendo posible la generación de modelos de gran complejidad. Sin embargo, tal y como ya hemos comentado, se ha observado una correlación deficiente entre las predicciones de los modelos computacionales y los resultados obtenidos a partir de estudios experimentales.

Las dificultades que surgen en el proceso de ajuste se encuentran asociadas tanto a inexactitudes en el modelo como a errores en los datos experimentales. Aunque los datos experimentales poseen errores que pueden provocar una baja correlación entre éstos y las predicciones, la teoría de ajuste de modelos supone que los principales causantes del desacuerdo teórico-experimental son los errores en el modelo. Según [42] se consideran tres tipos de error:

- Errores en el modelo de la estructura, los cuales se presentan cuando existe incertidumbre en las ecuaciones físicas gobernantes (como en el modelado de sistemas con un fuerte comportamiento no lineal).

- Errores en los parámetros del modelo, dentro de los que se encuentra la aplicación de condiciones de contorno inapropiadas, así como suposiciones imprecisas empleadas para simplificar el modelo.

- Errores en el orden del modelo, cuya aparición se debe a la discretización de sistemas complejos, la cual puede producir modelos de orden insuficiente.

El propósito del FEM Updating, en el caso que nos ocupa, es modificar los parámetros del modelo numérico con el fin de obtener un mayor ajuste entre sus resultados y la información experimental. Esta teoría surge como una derivación de la identificación de 
sistemas en ingeniería de control $^{1}$, con la diferencia de que el ajuste de modelos (cuyo objetivo es la generación de modelos con propósitos de predicción) exige que los parámetros tengan sentido físico.

Existen varias técnicas para ajustar modelos de elementos finitos, pudiéndose clasificar en métodos directos y métodos iterativos, dividiéndose éstos últimos en aquellos que emplean datos modales y aquellos que emplean FRFs.

Cuando se realiza el ajuste de un modelo analítico, es necesario tener la capacidad de comparar los resultados experimentales con los resultados obtenidos en el modelo de elementos finitos y a la hora de hacerlo uno de los mayores problemas que surge es la diferencia de grados de libertad entre ambos modelos. En general, el modelo de elementos finitos tiene un mayor número de grados de libertad debido a las limitaciones en lo que se refiere a los posibles puntos de medida experimentales. Debido a que el número de grados de libertad es distinto, las matrices de masa, rigidez y amortiguamiento tienen diferentes tamaños, imposibilitando la operatividad entre ellas. Para la resolución de este problema se puede, o bien reducir el modelo analítico (los grados de libertad del modelo analítico son reducidos o condensados al número de grados de libertad del modelo experimental, llamados nodos maestros), o bien expandir el modelo experimental (los grados de libertad del modelo experimental se expanden hasta el número de grados de libertad analíticos). Otro problema es la ausencia de amortiguamiento en el modelo de elementos finitos, de forma que el análisis modal experimental identifica modos de vibración complejos y este modelo se basa en modos normales.

Mientras los métodos directos modifican todos los elementos de las matrices de manera indiscriminada, los esquemas iterativos proporcionan un amplio abanico de posibilidades para seleccionar parámetros de ajuste, con los que el usuario puede centrarse en la corrección de aspectos físicos bien definidos y localizados.

\footnotetext{
${ }^{1}$ Construcción de modelos matemáticos de sistemas con base en datos experimentales
} 


\subsubsection{Métodos de optimización directa}

Son los métodos en los que los elementos individuales en las matrices del sistema son ajustados directamente por comparación entre los datos experimentales y la predicción del modelo analítico inicial.

Los métodos de optimización directa se basan en el uso de tres tipos de datos: los datos modales experimentales y las matrices de masa y rigidez del modelo analítico. Como premisa inicial se establece que uno de estos tipos de datos se conoce de forma precisa, ajustando los otros dos restantes a partir de él.

A su vez estos métodos se pueden dividir en: métodos de los multiplicadores de Lagrange -tratan de minimizar una función objetivo sujeta a ciertas restricciones en las variables independientes-, métodos basados en la Combinación de Matrices -toman como hipótesis que es posible medir todos los modos de vibración en todos los grados de libertad y por tanto construir las matrices de masa y rigidez directamente-, métodos de la Matriz Error -estiman directamente el error cometido en las matrices de masa y rigidez, suponiendo que éste es muy pequeño- [83].

\subsubsection{Métodos iterativos}

En general los datos modales que se emplean en estos métodos son las frecuencias naturales, los modos propios y los factores de amortiguamiento, siendo el objetivo mejorar la correlación entre los datos experimentales y los obtenidos a partir del modelo de elementos finitos mediante pequeños cambios en un grupo de parámetros del modelo [84].

La técnica más utilizada para correlacionar el modelo experimental y el modelo matemático es la comparación entre las frecuencias naturales, pudiéndose la diferencia en porcentaje expresarse del siguiente modo:

$$
\epsilon_{\omega i}=\frac{\left|\omega_{A i}-\omega_{X i}\right|}{\omega_{A i}} \times 100
$$

siendo

$\omega_{\mathrm{Xi}} \quad$ frecuencia experimental 
$\omega_{\mathrm{Ai}} \quad$ frecuencia calculada

Un indicador general de la dispersión de la frecuencia puede ser el siguiente:

$$
\epsilon_{\omega}=\left[\frac{\sum_{i=1}^{L}\left(\omega_{A i}-\omega_{X i}\right)^{2}}{\sum_{i=1}^{L} \omega_{A i}{ }^{2}}\right]^{1 / 2} \times 100
$$

donde $\mathrm{L}$ es el número de frecuencias naturales medidas.

En el caso de la correlación entre los modos de vibración experimentales y los estimados puede medirse a través del factor de escala modal (MSF - Modal Scale Factor), que representa la pendiente de la línea recta que mejor se ajusta entre ambos conjuntos de datos, esto es,

$$
\operatorname{MSF}\left(\Psi_{A}, \Psi_{X}\right)=\frac{\left\{\Psi_{A}\right\}^{T}\left\{\Psi_{X}\right\}^{*}}{\left\{\Psi_{A}\right\}^{T}\left\{\Psi_{A}\right\}^{*}}
$$

siendo

$\left\{\Psi_{X}\right\}$ modo de vibración experimental

$\left\{\Psi_{A}\right\} \quad$ modo de vibración calculado

donde la notación ${ }^{\mathrm{T}}$ significa matriz transpuesta y la notación ${ }^{*}$ significa compleja conjugada de la matriz.

Sin embargo, el criterio empleado frecuentemente para evaluar dicha correlación es el criterio de confianza modal (MAC - Modal Assurance Criterion).

Esta función proporciona una medida del grado de linealidad entre estimaciones de los modos de vibración originadas desde diferentes fuentes (valores cercanos a la unidad indican correspondencia entre ambos modos, mientras que los valores cercanos a cero indican que no se trata del mismo modo de vibración). Se define como un escalar y su expresión se muestra a continuación:

$$
M A C_{i j}=\frac{\left|\left\{\Psi_{A}\right\}_{i}^{H}\left\{\Psi_{X}\right\}_{j}\right|^{2}}{\left(\left\{\Psi_{A}\right\}_{i}^{H}\left\{\Psi_{A}\right\}_{i}\right)\left(\left\{\Psi_{X}\right\}_{j}^{H}\left\{\Psi_{X}\right\}_{j}\right)}
$$

donde la notación ${ }^{\mathrm{H}}$ significa transpuesta compleja conjugada (matriz Hermítica). ${ }^{2}$

\footnotetext{
${ }^{2}$ Valores cercanos a la unidad indican una buena correlación entre el modo propio experimental y el autovector calculado.
} 
Otra forma de expresar este criterio es el siguiente:

$$
M A C_{i j}=\frac{\left(\left\{\Psi_{A}\right\}_{i}^{T}\left\{\Psi_{\chi}\right\}_{j}^{*}\right)^{2}}{\left(\left\{\Psi_{A}\right\}_{i}^{T}\left\{\Psi_{A}\right\}_{i}^{*}\right)\left(\left\{\Psi_{X}\right\}_{j}^{T}\left\{\Psi_{X}\right\}_{j}^{*}\right)}
$$

Desde que se propuso este criterio hasta nuestros días se han formulado algunas variantes, entre las que se incluyen algunas que se aplican a los grados de libertad o que incorporan la matriz de masa o rigidez como matriz de peso en el cálculo [85].

Otro método se basa en expresar la relación entre los datos modales y los parámetros desconocidos en términos de un desarrollo en serie de Taylor limitada a los términos lineales, lo que puede ser escrito como:

$$
\delta \mathrm{z}=\mathrm{S}_{\mathrm{j}} \delta \theta
$$

donde

$\delta \theta=\theta-\theta_{j}$ es la perturbación en los parámetros

$\delta z=z_{m}-z_{j}$ es el error en los datos experimentales

$S_{j}=$ matriz de sensibilidad (que contiene la primera derivada de los autovalores y los vectores propios respecto a los parámetros).

Esta ecuación, que normalmente es indeterminada (porque se cuenta con más datos experimentales que parámetros desconocidos), puede ser resuelta usando una pseudoinversa de Moore-Penrose (mínimos cuadrados), mínimos cuadrados ponderados (si se emplean coeficientes de ponderación en los datos experimentales) o la técnica Bayesiana, (si se emplean coeficientes de ponderación en los datos experimentales y en los parámetros) [42, 43].

El uso de datos de Funciones de Respuesta en Frecuencia (FRF) no requiere realizar un análisis modal, por lo que se evitan errores de identificación, sin embargo estos métodos para ser utilizados requieren que el amortiguamiento sea incluido en el modelo de los elementos finitos.

El enfoque tradicional de ajuste con funciones de respuesta se basa en dos tipos de error denominados error de ecuaciones y error de salida. Ambos errores se basan en la ecuación de movimiento en el dominio de frecuencia [86]. 
En el caso de error en ecuaciones se minimiza el error en las ecuaciones de movimiento, y se expresa:

$$
\varepsilon_{\mathrm{ecu}}=\mathrm{F}(\omega)-\mathrm{B}(\omega) X(\omega)
$$

donde

$F(\omega)$ y $X(\omega)$ corresponden a los datos experimentales (generalmente se supone que la fuerza es unitaria en todas las frecuencias y se reemplaza $\mathrm{X}(\omega)$ por la FRF.

$\mathrm{B}(\omega)$ es la matriz de rigidez dinámica

En el caso de error de salida se minimiza la diferencia entre la respuesta medida y la estimada en el modelo numérico, y se expresa:

$$
\varepsilon_{\mathrm{sal}}=\mathrm{B}(\omega)^{-1} \mathrm{~F}(\omega)-\mathrm{X}(\omega)
$$

Como apunte final a la revisión de los diferentes métodos de ajuste/actualización cabe mencionar que existen otros enfoques en la estimación de la incertidumbre de los parámetros, cuya principal diferencia radica en el uso de herramientas de análisis probabilístico [87] para hallar el cambio en los parámetros que maximiza la probabilidad de que las predicciones concuerden con los datos experimentales, en lugar de minimizar el error de predicción. 



\section{CAPÍTULO 4. MÉTODOS DE ESCALADO PROPUESTOS}


La técnica de análisis modal consiste en la recolección de datos, la identificación del sistema y por último, la estimación de los parámetros modales (frecuencia, amortiguamiento, masa modal y modos de vibración). La etapa de identificación del sistema juega un papel crucial en los parámetros modales que se derivan, así como en el número de parámetros que se pueden determinar [45]. Los métodos de identificación clásicos se basan en aplicar una excitación al sistema y a continuación, registrar la correspondiente respuesta [46].

El método de identificación más utilizado en la actualidad se denomina Stochastic Subspace Identification (SSI) [90-93] que se resume en el apartado 4.1. Sin embargo, la información modal que proporcionan los métodos de identificación, incluido el SSI, es incompleta ya que proporcionan estimaciones de frecuencias, factores de amortiguamiento y modos de vibración asociados, pero dichos modos no están normalizados respecto de la matriz de masa o dicho de otra forma, esos métodos no calculan las masas generalizadas correspondientes a cada uno de los modos estimados (masas modales) [47] y el modelo resulta incompleto lo que no permite la simulación dinámica del sistema.

Para evitar este problema, se han desarrollado algunos métodos de escalado de los modos de vibración que se basan en la actualización de un modelo de elementos finitos de la estructura empleando aquellos parámetros modales estimados de forma experimental [42, 48].

También se han desarrollado soluciones basadas en la suposición de que la matriz de masa de la estructura (o su inversa) es conocida [49] o modificando el comportamiento dinámico de la estructura variando la masa o la rigidez y realizando un análisis modal operacional tanto en la estructura original como en la modificada [50-56]. Estos métodos requieren un procedimiento experimental extenso al emplear los parámetros modales de la estructura original y también los de la modificada.

Otros métodos de escalado consisten en añadir sistemas de vibración secundarios midiendo y procesando la respuesta conjunta [44].

En el presente trabajo se estudian dos técnicas adicionales que a partir de toda la información obtenida al aplicar el método SSI, con una mínima información experimental 
respecto a la entrada y sin necesidad de modelos computacionales complejos, nos permiten estimar todos los parámetros modales, incluidas las masas modales o los modos normalizados asociados a cada modo de vibración:

- Una de estas técnicas consiste en la resolución de un problema simétrico de valores propios [67, 94-95], siendo un requisito mínimo el que en todos los grados de libertad se debe ubicar o bien un sensor o un actuador, con al menos una localización de ubicación conjunta (par sensor-actuador), lo que proporciona una solución para el problema inverso. Esta técnica se describe con más detalle en el apartado 4.2.

- Otra de las técnicas consiste en escalar los modos de vibración a partir de la estimación de los residuos de la descomposición en fracciones parciales de la FRF de las mediciones [96]. Esta técnica se describe con más detalle en el apartado 4.3. 


\subsection{Stochastic Subspace Identification}

Una de las formulaciones bien conocidas para los sistemas dinámicos es la expresión matricial de la segunda ley de Newton, que una vez discretizada en el dominio espacial resulta:

$$
\boldsymbol{M} \ddot{\boldsymbol{u}}(t)+\boldsymbol{C} \dot{\boldsymbol{u}}(t)+\boldsymbol{K} \boldsymbol{u}(t)=\boldsymbol{f}(t) ; \quad \boldsymbol{y}(t)=\left\{\begin{array}{c}
\boldsymbol{y}_{\mathbf{1}}(\boldsymbol{t}) \\
\ldots \\
\boldsymbol{y}_{\boldsymbol{M}}(\boldsymbol{t})
\end{array}\right\}
$$

donde:

$\boldsymbol{u}(t) \quad$ Vector desplazamiento del sistema, con (') y (“) que representa la primera y segunda derivada respecto al tiempo respectivamente

M Matriz de masas

C Matriz de amortiguamiento

K Matriz de rigidez

$\boldsymbol{f}(t) \quad$ Vector que contiene las excitaciones externas que solicitan el sistema

$\boldsymbol{y}(t) \quad$ Vector respuesta del sistema

Esta ecuación (4-1) en tiempo continuo puede ser transformada mediante una formulación en el espacio de estados del siguiente modo:

$$
\begin{aligned}
& \dot{\boldsymbol{x}}(t)=\boldsymbol{A}_{\boldsymbol{C}} \boldsymbol{x}(t)+\boldsymbol{B}_{\boldsymbol{C}} \boldsymbol{f}(t) \\
& \boldsymbol{y}(t)=\boldsymbol{C}_{\boldsymbol{C}} \boldsymbol{x}(t)+\boldsymbol{D}_{\boldsymbol{C}} \boldsymbol{f}(t)
\end{aligned}
$$

Siendo

$$
\boldsymbol{x}(t)=\left[\begin{array}{l}
\boldsymbol{u}(t) \\
\dot{\boldsymbol{u}}(t)
\end{array}\right]
$$

y donde $\boldsymbol{A}_{\boldsymbol{C}}, \boldsymbol{B}_{\boldsymbol{C}}$, y $\boldsymbol{C}_{\boldsymbol{C}}$ son las matrices del sistema en tiempo continuo:

$$
\begin{gathered}
\boldsymbol{A}_{C}=\left[\begin{array}{cc}
0 & I \\
-M^{-1} K & -M^{-1} C
\end{array}\right] \\
\boldsymbol{B}_{C}=\left[\begin{array}{c}
0 \\
M^{-1}
\end{array}\right]
\end{gathered}
$$


$\boldsymbol{C}_{\boldsymbol{C}}$ es la matriz de salida y $\boldsymbol{D}_{\boldsymbol{C}}$ es la matriz de retroalimentación (cero en el caso de sistemas mecánicos) [97].

La ventaja de esta formulación es que la solución general se obtiene directamente:

$$
\boldsymbol{x}(t)=\exp \left(\boldsymbol{A}_{\boldsymbol{c}} t\right) \boldsymbol{x}(0)+\int_{0}^{t} \exp \left(\boldsymbol{A}_{\boldsymbol{c}}(t-\tau)\right) \boldsymbol{B}_{\boldsymbol{c}} \boldsymbol{f}(\tau) d \tau
$$

donde el primer término es la solución de la ecuación homogénea y el último término es la solución particular. Para discretizar en el dominio del tiempo esta solución, empleamos la transformación $\boldsymbol{u}_{K}=\boldsymbol{u}(k \Delta t)$ de modo que la solución se convierte en:

$$
\begin{gathered}
\boldsymbol{x}(t)=\exp \left(\boldsymbol{A}_{\boldsymbol{c}} k \Delta t\right) \boldsymbol{x}(0)=\boldsymbol{A}_{\boldsymbol{d}}^{k} \boldsymbol{x}_{0} \\
\boldsymbol{A}_{d}=\exp \left(\boldsymbol{A}_{c} \Delta t\right) \\
\boldsymbol{y}_{k}=C_{c} \boldsymbol{A}_{d}^{k} \boldsymbol{x}_{0}
\end{gathered}
$$

Para obtener los parámetros modales del sistema es necesario descomponer la matriz del sistema discreto $\boldsymbol{A}_{\boldsymbol{d}}$ en :

$$
\boldsymbol{A}_{d}=\phi \Lambda \phi^{-1}
$$

donde

$\phi$ contiene los autovectores del modelo de espacio de estados y $\boldsymbol{\Lambda}$ es la matriz diagonal de los valores propios $\left(\mu_{1}, \ldots . \mu_{n}\right)$.

Los valores propios del sistema continuo, que denominaremos $\lambda_{i}$, se obtienen a partir de los valores propios discretos $\left(\mu_{i}\right)$ del siguiente modo:

$$
\lambda_{i}=\frac{\ln \left(\mu_{i}\right)}{\Delta T}
$$

Los autovectores del sistema $(\phi)$ son los mismos tanto para la representación del espacio de estados discreto como continuo.

Las frecuencias naturales sin amortiguamiento $\left(\omega_{i}\right)$ y los coeficientes/razones de amortiguamiento $\left(\xi_{i}\right)$ se obtienen teniendo en cuenta que

$$
\lambda_{i}=\sigma_{i} \pm j \omega_{D i}
$$

donde 
$\sigma_{\text {i }}$ se denomina factor de amortiguamiento

$\omega_{D i}$ se denomina frecuencia amortiguada

$\mathrm{Y}$ teniendo en cuenta que $\xi_{i}=\frac{-\sigma_{i}}{\sqrt{\omega_{D i}^{2}+\sigma_{i}^{2}}} \quad$ y $\quad \omega_{i}=\frac{-\sigma_{i}}{\xi_{i}}$

$$
\lambda_{i}=\left(-\xi_{i} \pm j \sqrt{\left(1-\xi_{i}^{2}\right)}\right) \omega_{i}
$$

Del siguiente modo

$$
\begin{gathered}
\omega_{i}=\left|\lambda_{i}\right| \\
\xi_{i}=\frac{\operatorname{Re}\left(\lambda_{i}\right)}{\left|\lambda_{i}\right|}
\end{gathered}
$$

y los modos de vibración $\left(\varphi_{\mathrm{i}}\right)$ del sistema

$$
\Phi=C_{c} \boldsymbol{\varphi}
$$




\subsection{Problema simétrico de valores propios}

El problema dinámico se puede expresar en forma matricial del siguiente modo:

$$
\begin{gathered}
\boldsymbol{M} \ddot{\boldsymbol{u}}(t)+\boldsymbol{C} \dot{\boldsymbol{u}}(t)+\boldsymbol{K} \boldsymbol{u}(t)=\boldsymbol{f}(t) ; \\
\boldsymbol{y}(t)=\ddot{\boldsymbol{u}}(t)
\end{gathered}
$$

Siendo $\boldsymbol{y}(t)$ al vector respuesta del sistema que para el caso anterior representa las mediciones de las aceleraciones.

Si definimos el vector de estados $\boldsymbol{x}(t)=\left[\begin{array}{c}\boldsymbol{u}(t) \\ \dot{\boldsymbol{u}}(t)\end{array}\right]$, el sistema anterior puede reescribirse como

$$
\begin{aligned}
& {\left[\begin{array}{cc}
\boldsymbol{C} & \boldsymbol{M} \\
\boldsymbol{M} & \mathbf{0}
\end{array}\right] \dot{\boldsymbol{x}}(t)+\left[\begin{array}{cc}
\boldsymbol{K} & \mathbf{0} \\
\mathbf{0} & -\boldsymbol{M}
\end{array}\right] \boldsymbol{x}(t)=\left[\begin{array}{l}
\boldsymbol{I} \\
\mathbf{0}
\end{array}\right] \boldsymbol{f}(t)} \\
& \boldsymbol{y}(t)=\left[\begin{array}{ll}
0 & I
\end{array}\right] \dot{\boldsymbol{x}}(t) \quad \text { para aceleraciones } \\
& \boldsymbol{y}(t)=\left[\begin{array}{ll}
0 & I
\end{array}\right] \boldsymbol{x}(t) \quad \text { para velocidades } \\
& \boldsymbol{y}(t)=\left[\begin{array}{ll}
I & 0
\end{array}\right] \boldsymbol{x}(t) \quad \text { para desplazamientos }
\end{aligned}
$$

La ventaja de esta nueva expresión (ecuación 4-20) es que ahora el problema de autovalores (valores propios) asociado es simétrico y puede plantearse como:

$$
\left[\begin{array}{cc}
\boldsymbol{C} & \boldsymbol{M} \\
\boldsymbol{M} & \mathbf{0}
\end{array}\right]\left[\begin{array}{c}
\Psi \\
\Psi \Lambda
\end{array}\right] \Lambda=\left[\begin{array}{cc}
-\boldsymbol{K} & \mathbf{0} \\
\mathbf{0} & M
\end{array}\right]\left[\begin{array}{c}
\Psi \\
\Psi \Lambda
\end{array}\right]
$$

donde $\Psi=\left[\begin{array}{lll}\Psi_{1} & \ldots & \Psi_{N}\end{array}\right]$ es la matriz que contiene los vectores propios del problema simétrico complejo.

$$
\left(\lambda_{i}^{2} \boldsymbol{M}+\lambda_{i} \boldsymbol{C}+\boldsymbol{K}\right) \Psi_{i}=0
$$

y $\boldsymbol{\Lambda}$ es la matriz diagonal de los valores propios del problema simétrico complejo $\lambda_{i}$.

En general, los vectores propios pueden escalarse de forma arbitraria pero si los escalamos del siguiente modo [45, 98]:

$$
\left[\begin{array}{c}
\Psi \\
\Psi \Lambda
\end{array}\right]^{T}\left[\begin{array}{cc}
C & M \\
M & 0
\end{array}\right]\left[\begin{array}{c}
\Psi \\
\Psi \Lambda
\end{array}\right]=I
$$




$$
\left[\begin{array}{c}
\Psi \\
\Psi \Lambda
\end{array}\right]^{T}\left[\begin{array}{cc}
K & 0 \\
0 & -M
\end{array}\right]\left[\begin{array}{c}
\Psi \\
\Psi \Lambda
\end{array}\right]=-\Lambda
$$

Llegamos a las siguientes expresiones que nos permiten calcular las matrices de masa, amortiguamiento y rigidez del sistema

$$
\begin{aligned}
& \boldsymbol{M}=\left(\Psi \boldsymbol{\Lambda} \Psi^{T}\right)^{-1} \\
& \boldsymbol{C}=-\boldsymbol{M} \Psi \boldsymbol{\Lambda}^{2} \Psi^{T} \boldsymbol{M} \\
& \boldsymbol{K}=-\left(\Psi \boldsymbol{\Lambda}^{-1} \Psi^{T}\right)^{-1}
\end{aligned}
$$

Si realizamos una nueva transformación $x(t)=\left[\begin{array}{c}\Psi \\ \Psi \Lambda\end{array}\right] z(t)$ las ecuaciones (4-20), (4-21), (4-22), (4-23) pueden expresarse como se muestra a continuación

$$
\begin{array}{ll}
\qquad \dot{\boldsymbol{z}}(t)=\boldsymbol{\Lambda z}(t) & +\Psi^{T} \boldsymbol{f}(t) \\
\boldsymbol{y}(t)=\Psi \Lambda^{2} \boldsymbol{z}(t)+\Psi \boldsymbol{\Lambda}^{T} \boldsymbol{f}(t) & \text { para aceleraciones } \\
\boldsymbol{y}(t)=\Psi \Lambda \boldsymbol{z}(t) & \text { para velocidades } \\
\boldsymbol{y}(t)=\Psi \boldsymbol{z}(t) & \text { para desplazamientos }
\end{array}
$$

Denominaremos al sistema definido por las ecuaciones anteriores, modelo modal simétrico. Al sistema definido por las ecuaciones (4-2) y (4-3) lo denominaremos modelo modal no simétrico.

Si en este punto retomamos el modelo modal no simétrico y realizamos la siguiente transformación $\boldsymbol{x}(t)=\boldsymbol{\phi} \boldsymbol{\theta}(t)$ (recordando que $\boldsymbol{\phi}$ contiene los autovectores del sistema y que Dc es cero para sistemas mecánicos como son los que nos ocupan en este documento), obtenemos las siguientes ecuaciones

$$
\begin{gathered}
\dot{\boldsymbol{\theta}}(t)=\boldsymbol{\Lambda \boldsymbol { \theta }}(t)+\boldsymbol{\varphi}^{-\mathbf{1}} \boldsymbol{B}_{\boldsymbol{C}} \boldsymbol{f}(t) \\
\boldsymbol{y}(t)=\boldsymbol{C}_{\boldsymbol{C}} \boldsymbol{\varphi} \boldsymbol{\theta}(t)
\end{gathered}
$$

Si consideramos los modelos modales simétrico y no simétrico como representaciones diferentes del mismo sistema dinámico, podemos buscar una matriz diagonal de transformación $(\boldsymbol{T})$, que relacione ambos modelos, es decir:

$$
\boldsymbol{\theta}(t)=\boldsymbol{T} \boldsymbol{z}(t)
$$


Obteniendo las siguientes igualdades

$$
\begin{array}{cc} 
& T \Lambda T^{-1}=\Lambda \\
C_{C} \varphi T=\Psi \Lambda^{2} & T^{-1} \varphi^{-1} B_{C}=\Psi^{T} \\
C_{C} \varphi T=\Psi \Lambda & \text { para aceleraciones } \\
C_{C} \varphi T=\Psi & \text { para velocidades } \\
\end{array}
$$

Si suponemos que el grado de libertad i-ésimo contiene un sensor y un actuador, el requisito de co-localización junto con el hecho de que la matriz $\boldsymbol{T}$ es diagonal, permite determinar dicha matriz de manera unívoca mediante la siguiente expresión:

En el caso de medir aceleraciones

$$
C_{C}(\mathrm{i},:) \boldsymbol{\varphi} \Lambda^{-2} \mathbf{T}^{2}=\left(\boldsymbol{\varphi}^{-1} \mathbf{B}_{\mathbf{C}}\right)^{\mathrm{T}}
$$

En el caso de medir velocidades

$$
\begin{aligned}
& C_{C}(\mathrm{i},:) \boldsymbol{\varphi} \Lambda^{-1} \mathbf{T}^{2}=\left(\boldsymbol{\varphi}^{-1} \mathbf{B}_{\mathrm{C}}\right)^{\mathrm{T}} \\
& \boldsymbol{C}_{C}(i,:) \boldsymbol{\varphi} \mathbf{T}^{2}=\left(\boldsymbol{\varphi}^{-1} \boldsymbol{B}_{C}\right)^{T}
\end{aligned}
$$

Dependiendo de la configuración de sensores y actuadores la información relativa a un cierto grado de libertad está embebida ya sea en la matriz de entrada o en el matriz de salida, por lo que para obtener los vectores propios del modelo modal simétrico $(\boldsymbol{\Psi})$ para cada grado de libertad $(i)^{4}$, emplearemos las ecuaciones (4-40) ó (4-41) ó (4-42) en el caso de tener un sensor (que mida aceleraciones ó velocidades ó desplazamientos respectivamente) y la expresión (4-39) en el caso de tener un actuador. Es decir,

$$
C_{C}(i,:) \varphi T=\Psi(i,:) \Lambda^{2}
$$

en el caso de tener un sensor que mida aceleraciones

$$
C_{C}(i,:) \varphi T=\Psi(i,:) \Lambda
$$

en el caso de tener un sensor que mida velocidades

$$
C_{C}(i,:) \varphi \boldsymbol{T}=\Psi(i,:)
$$

\footnotetext{
3 (i,:) denota la fila i de la matriz correspondiente

${ }^{4}$ Una vez obtenida la matriz de transformación $(\boldsymbol{T})$
} 
en el caso de tener un sensor que mida desplazamientos

$$
\boldsymbol{T}^{-1} \boldsymbol{\varphi}^{-\mathbf{1}} \boldsymbol{B}_{C}(i,:)=\Psi(i,:)^{T}
$$

en el caso de tener un actuador

Finalmente, a partir de los vectores propios simétricos $(\boldsymbol{\Psi})$ podemos obtener la matriz de masa $(\boldsymbol{M})$ (y así poder calcular los modos normalizados) mediante la ecuación (4-28) y calcular las matrices de amortiguamiento $(\boldsymbol{C})$ y rigidez $(\boldsymbol{K})$ mediante las ecuaciones (429) y (4-30). 


\subsection{Escalado de los modos de vibración a partir de la función de respuesta en frecuencia}

La función de transferencia de un sistema amortiguado de múltiples grados de libertad puede ser formulada a partir de las ecuaciones del movimiento en términos de las matrices de masa, rigidez y amortiguamiento.

$$
\boldsymbol{M} \ddot{\boldsymbol{u}}(t)+\boldsymbol{C} \dot{\boldsymbol{u}}(t)+\boldsymbol{K u}(t)=\boldsymbol{f}(t)
$$

Aplicando la transformada de Laplace a la ecuación anterior, y asumiendo condiciones iniciales nulas, resulta

$$
\left(s^{2} \boldsymbol{M}+s \boldsymbol{C}+\boldsymbol{K}\right) \boldsymbol{U}(s)=\boldsymbol{F}(s)
$$

donde $\boldsymbol{U}(s)=\int_{0}^{\infty} e^{-s t} \boldsymbol{u}(t) d t$ es la transformada de Laplace de los desplazamientos y $\quad \boldsymbol{F}(s)=\int_{0}^{\infty} e^{-s t} \boldsymbol{f}(t) d t$ es la transformada de Laplace de la fuerza y $s$ es una variable subsidiaria, en este caso $s=j \omega$.

Si se define la matriz dinámica del sistema, como:

$$
\boldsymbol{B}(s)=\left(s^{2} \boldsymbol{M}+s \boldsymbol{C}+\boldsymbol{K}\right)
$$

La ecuación (2-116) se reescribe como

$$
\boldsymbol{B}(s) \boldsymbol{U}(s)=\boldsymbol{F}(s)
$$

O lo que es lo mismo

$$
\boldsymbol{U}(s)=\boldsymbol{B}(s)^{-1} \cdot \boldsymbol{F}(s)
$$

Y podemos definir la matriz función de transferencia o función compleja de respuesta en frecuencia $(\boldsymbol{H}(s)$ ) (que relaciona la respuesta del sistema con las fuerzas excitadoras de la misma) a partir de la matriz dinámica del siguiente modo:

$$
\boldsymbol{H}(s)=[\boldsymbol{B}(s)]^{-1}=\boldsymbol{U}(s) \cdot[\boldsymbol{F}(s)]^{-1}
$$

En general

$$
H_{p q}(s)=\frac{U_{p}(s)}{F_{q}(s)}
$$


donde $p$ es el grado de libertad de salida y $q$ es el grado de libertad de entrada (orientación y posición física $)^{5}$.

De este modo, la obtención de una columna de la matriz función de transferencia $(\boldsymbol{H}(s))$ se lleva a cabo midiendo la respuesta dinámica en distintos puntos $\left(U_{p}\right)$ manteniendo un excitador en una posición fija $\left(F_{\mathrm{q}}\right)$. También se puede obtener la fila de la matriz función de transferencia $\boldsymbol{- H}(s)$ - midiendo la respuesta dinámica en un punto fijo $\left(U_{p}\right)$ desplazando el excitador a distintos puntos $\left(F_{q}\right)$.

Otra forma de expresar cada uno de los términos de la matriz función de transferencia, usando coeficientes escalares $(\alpha$ y $\beta$ ), es la siguiente:

$$
H_{p q}(s)=\frac{\beta_{n}(s)^{n}+\beta_{n-1}(s)^{n-1}+\cdots+\beta_{1}(s)^{1}+\beta_{0}(s)^{0}}{\alpha_{m}(s)^{m}+\alpha_{m-1}(s)^{m-1}+\cdots+\alpha_{1}(s)^{1}+\alpha_{0}(s)^{0}}
$$

Siendo las raíces del polinomio del denominador los denominados polos de la función de transferencia o autovalores $(\lambda)$, dicho denominador puede expresarse como producto de sus raíces y también puede descomponerse en fracciones parciales, tal y como se indica a continuación:

$$
H_{p q}(s)=\sum_{i=1}^{N} \frac{\left[A_{p q i}\right]}{\left(s-\lambda_{i}\right)}+\frac{\left[A_{p q i}^{*}\right]}{\left(s-\lambda_{i}^{*}\right)}
$$

donde, $i$ es el número de modos, $A_{p q i}$ son los residuos asociados al polo (autovalor) $\lambda_{i}, \mathrm{y}$ ()* indica el complejo conjugado.

Dichos residuos pueden expresarse en función de los modos de vibración $(\boldsymbol{\phi})$ del siguiente modo:

$$
A_{p q i}=Q_{i} \phi_{p i} \phi_{q i}
$$

siendo $Q_{i}$ el coeficiente de escalado del modo de vibración $\phi_{i}$.

Por ello,

$$
H_{p q}(s)=\sum_{i=1}^{N} \frac{Q_{i} \phi_{p i} \phi_{q i}}{\left(s-\lambda_{i}\right)}+\frac{Q_{i}^{*} \phi_{p i}^{*} \phi_{q i}^{*}}{\left(s-\lambda_{i}^{*}\right)}
$$

\footnotetext{
${ }^{5}$ El modelo matemático que se describe corresponde a la medida de desplazamientos, en el caso de que se midan velocidades, es necesario que los datos velocidad/fuerza se dividan entre $j \omega$. Si se miden aceleraciones, los datos aceleración/fuerza deben dividirse entre $(j \omega)^{2}$.
} 
Teniendo en cuenta todo lo anterior se puede establecer una relación entre el residuo y la masa modal, distinguiendo dos casos:

- Amortiguamiento proporcional (incluye el caso sin amortiguamiento).

- Amortiguamiento no proporcional.

\section{Amortiguamiento proporcional}

Para el primer caso (amortiguamiento proporcional) dicha relación se obtiene teniendo en cuenta que $B[s] \cdot H[s]=[I]^{6}$ y $A_{p q i}=Q_{i} \phi_{p i} \phi_{q i}$. Realizando varias operaciones matemáticas [96] se llega a la siguiente expresión

$$
M_{i}=\frac{1}{2 j \omega_{D i} Q_{i}}=\frac{\phi_{p i} \phi_{q i}}{j 2 A_{p q i} \omega_{D i}}
$$

recordando que $\omega_{D i}$ es la frecuencia natural amortiguada para el modo $i$.

Como es sabido, los modos de vibración pueden escalarse de varias formas, pero escogiendo el escalado de forma que el mayor coeficiente modal sea igual a 1 se obtiene un resultado en el que la masa calculada según la ecuación (4-63) tiene un significado físico. Este significado físico es que la cantidad de masa calculada bajo estas condiciones será un número entre 0 y la masa total del sistema que puede verse como la cantidad de masa que participa en cada modo de vibración (masa modal).

Para obtener el valor de $Q_{i}$, una vez escalados los modos de vibración, sólo es necesario disponer de un grado de libertad (por ejemplo $q$ ) en el que dispongamos de un sensor y un actuador (dicho punto se denomina driving point de la FRF) ya que se cumple que,

$$
A_{q q i}=Q_{i} \phi_{q i} \phi_{q i}=Q_{i} \phi_{q i}^{2}
$$

Una vez que la masa modal es conocida, se puede obtener el amortiguamiento modal y la rigidez modal del siguiente modo:

$$
\begin{aligned}
& C_{i}=2 \sigma_{i} M_{i} \\
& K_{i}=\left(\sigma_{i}^{2}+\omega_{D i}{ }^{2}\right) M_{i}=\omega_{i}{ }^{2} M_{i}
\end{aligned}
$$

\footnotetext{
${ }^{6}[\mathrm{I}]$ es la matriz identidad
} 


\section{Amortiguamiento no proporcional}

En un sistema con amortiguamiento no proporcional, las ecuaciones del movimiento se acoplan cuando se formulan en el espacio físico de dimensión $N$. Sin embargo, las ecuaciones del movimiento pueden ser desacopladas cuando se formulan en el espacio de estados de dimensión $2 N$.

Si retomamos el problema de autovalores simétrico y la ecuación (4-22) y denominamos:

$$
\boldsymbol{A}=\left[\begin{array}{cc}
\boldsymbol{C} & \boldsymbol{M} \\
\boldsymbol{M} & \mathbf{0}
\end{array}\right]
$$

$\mathrm{y}$

$$
\boldsymbol{B}=\left[\begin{array}{cc}
K & \mathbf{0} \\
\mathbf{0} & -\boldsymbol{M}
\end{array}\right]
$$

Podemos expresar un conjunto de relaciones de ortogonalidad para las matrices del sistema (en este caso $\boldsymbol{A}$ y $\boldsymbol{B}$ ) similar al caso de sistemas sin amortiguamiento.

$$
\begin{aligned}
& \boldsymbol{\psi}_{i}^{T} \boldsymbol{A} \boldsymbol{\psi}_{j}=0 \\
& \boldsymbol{\psi}_{i}^{T} \boldsymbol{B} \boldsymbol{\psi}_{j}=0 \\
& \boldsymbol{\psi}_{i}^{T} \boldsymbol{A} \boldsymbol{\psi}_{i}=M_{A i} \\
& \boldsymbol{\psi}_{i}^{T} \boldsymbol{B} \boldsymbol{\psi}_{i}=M_{B i}
\end{aligned}
$$

Siendo $\boldsymbol{\psi}$ los modos de vibración del modelo de espacio de estados de dimensión $2 \mathrm{~N}$ $\left(\psi=\left[\begin{array}{c}\phi \\ \lambda \phi\end{array}\right]\right), \quad M_{A i}$ un término que se denomina modal $A$ y $M_{B i}$ otro término que se denomina modal $B$ y que tienen las mismas propiedades respectivamente que la masa modal y la rigidez modal para los casos sin amortiguamiento y con amortiguamiento proporcional.

Siempre que los vectores modales (modos de vibración) sean complejos, las magnitudes modal A y modal B deben ser utilizadas para proporcionar el escalado modal en el caso de sistemas con amortiguamiento no proporcional, cumpliéndose que

$$
M_{i}=\frac{M_{A i}}{j 2 \omega_{D i}}
$$




$$
M_{A i}=\frac{1}{Q_{i}}=\frac{\phi_{p i} \phi_{q i}}{A_{p q i}}
$$



CAPÍTULO 5. APLICACIÓN Y RESULTADOS 
El capítulo se estructura en dos apartados; en el primero de ellos se describe el proceso de modelización y ajuste de la pasarela completa y los resultados obtenidos, y en el segundo se describe la validación de las técnicas de escalado descritas en el capítulo anterior y los resultados alcanzados al aplicarlas a la pasarela objeto de estudio.

En el caso de la modelización de la pasarela completa, se comparan las primeras frecuencias verticales de vibración y formas modales obtenidas numéricamente, con las presentadas en un trabajo de tesis anterior [2] y que sirven de referencia.

En el caso de las técnicas de escalado propuestas se procede a su validación aplicándolas a dos casos teóricos y posteriormente se obtienen las primeras frecuencias verticales, las masas modales y los modos de vibración normalizados. 


\subsection{Resultados de la modelización de la Pasarela del Museo de la Ciencia}

\subsubsection{Modelado de la pasarela}

La PMC es una estructura en servicio situada en Valladolid sobre el río Pisuerga. Dicha estructura, concebida como una cesta de pescar, está formada por cuatro vanos, tres de celosías metálicas formando una sección transversal hexagonal y un vano de hormigón blanco. Éste último es muy rígido por lo que no se considera en el análisis objeto del presente trabajo.

La estructura del tramo metálico de la pasarela (vanos 2, 3 y 4) consiste en una celosía tubular tridimensional de sección transversal hexagonal, que en el vano 3 se sitúa en el interior de otra celosía formada por cables pretensados longitudinales y transversales. Ambas celosías se enlazan mediante radios tubulares que permiten mantener la forma de los cables y aumentar su excentricidad en el centro del vano. La sección transversal es un hexágono irregular cuya inclinación y dimensiones cambian a lo largo del puente [88].

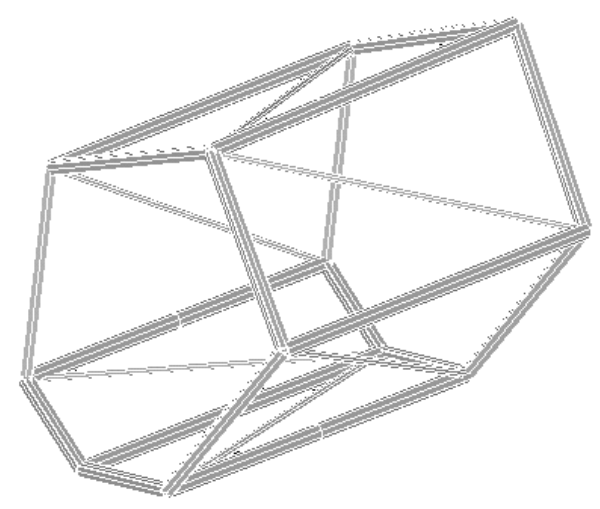

Figura 5-1. Celosía tubular tridimensional de sección transversal hexagonal.

En cuanto al pretensado transversal, cada sección hexagonal del vano 3 (son 15) consta de un tendón periférico en forma de hexágono, cuyos vértices son los extremos de los seis radios. El pretensado longitudinal consta de seis tendones que pasan por los extremos de los radios de las secciones transversales [89].Se ha modelizado la estructura de forma que los nudos que conforman tanto el hexágono exterior y el interior sean coplanarios. 


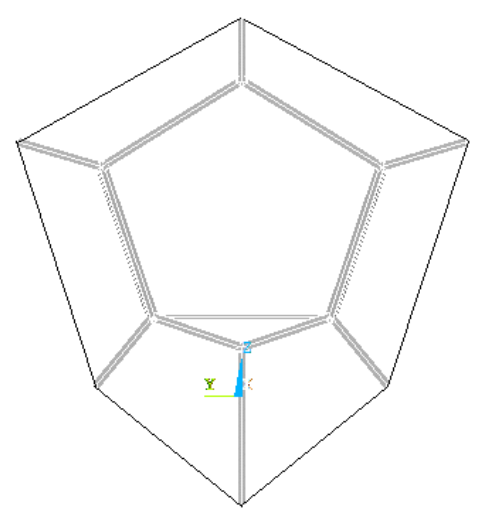

Figura 5-2. Sección transversal del pretensado del vano 3.

La estructura principal está formada por perfiles tubulares de sección circular. El diámetro de los perfiles longitudinales es de $273 \mathrm{~mm}$ y las diagonales son perfiles de diámetro 135 mm. Los montantes y radios son perfiles tubulares de sección cuadrada de dimensiones $200 \mathrm{~mm} \times 200 \mathrm{~mm}$.

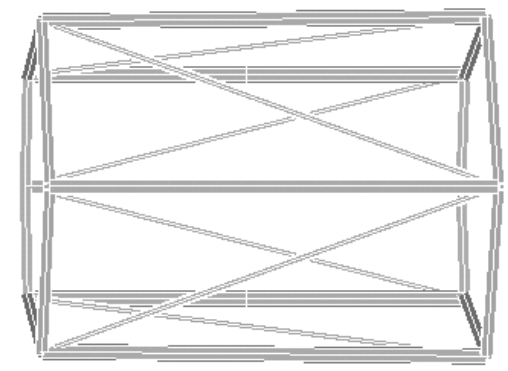

Figura 5-3. Estructura principal.

Las secciones de la estructura del tablero, constituida por travesaños y elementos de arriostramientos, son cuadradas de dimensiones $120 \mathrm{~mm}$ x $100 \mathrm{~mm}$ y $120 \mathrm{~mm}$ x $120 \mathrm{~mm}$ respectivamente.

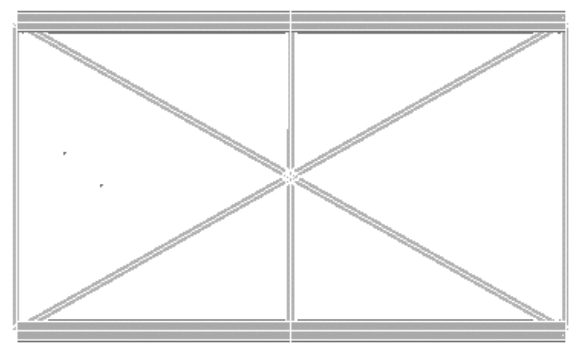

Figura 5-4. Estructura del tablero. 
Los apoyos centrales son dos torres constituidas por celosías resueltas también con perfiles tubulares de $355 \mathrm{~mm}$ de diámetro.

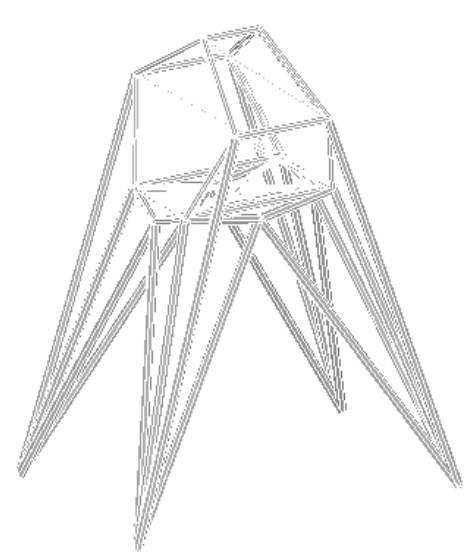

Figura 5-5. Apoyo central.

En cuanto a las propiedades del acero se ha considerado un módulo de elasticidad de $2,1 \cdot 10^{11} \mathrm{~N} / \mathrm{m}^{2}$, un coeficiente de Poisson igual a 0,3 y una densidad de $7.700 \mathrm{Kg} / \mathrm{m}^{3}$.

La modelización de la estructura se ha realizado mediante el programa ANSYS mediante dos tipos de elementos: BEAM188 para simular los perfiles tubulares, definiendo para cada tipo las secciones indicadas anteriormente y LINK180 para los tendones de pretensado. En el caso del pretensado longitudinal se ha impuesto que los movimientos en la dirección lateral y vertical de los nudos que lo modelizan sean iguales a los de los nudos que representan los extremos de los radios de las secciones transversales. La fuerza de pretensado se ha modelizado mediante el comando INISTATE.

La principal dificultad en la definición geométrica de la pasarela ha sido la modelización del pretensado, principalmente por la elección del tipo de elemento y de las conexiones de éste con el resto de la estructura con el fin de que la transmisión de esfuerzos se realizara de forma realista.

En cuanto a los apoyos, la estructura se considera apoyada en las torres centrales y empotrada en los extremos del tablero. Las bases de las torres se suponen empotradas. 


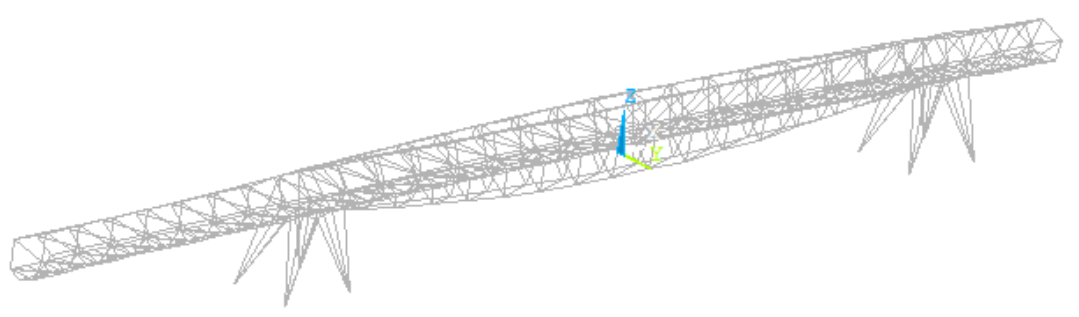

Figura 5-6. Vista general del modelo completo de la pasarela en ANSYS.

Se ha seleccionado para el análisis modal realizado el método de Block Lanczos.

\subsubsection{FEM Updating}

A continuación se exponen los resultados que se han extraído de un estudio anterior [2] y que se emplearán para el ajuste del modelo de elementos finitos de la pasarela. En el caso del OMA realizado en el mismo, los parámetros modales se identificaron empleando tres algoritmos en el dominio de la frecuencia: FDD (Frequency Domain Decomposition), EFDD (Enhanced Frequency Domain Decomposition) y CFDD (Curve-Fit Frequency Domain Decomposition). Se obtuvieron los siguientes resultados [2]:

\begin{tabular}{ccc}
\hline FDD & EFDD & CFDD \\
\hline Frecuencia $(\mathbf{H z})$ & Frecuencia $(\mathbf{H z})$ & Frecuencia $(\mathbf{H z})$ \\
\hline 1.245 & 1.243 & 1.257 \\
2.759 & 2.763 & 2.765 \\
3.516 & 3.514 & 3.515 \\
4.785 & 4.792 & 4.792 \\
\hline
\end{tabular}

Tabla 5-1. Valores de frecuencia de los primeros modos de flexión de la pasarela obtenidos con el OMA.

En la siguiente figura se muestran los cuatro primeros modos de la pasarela completa [2]: 


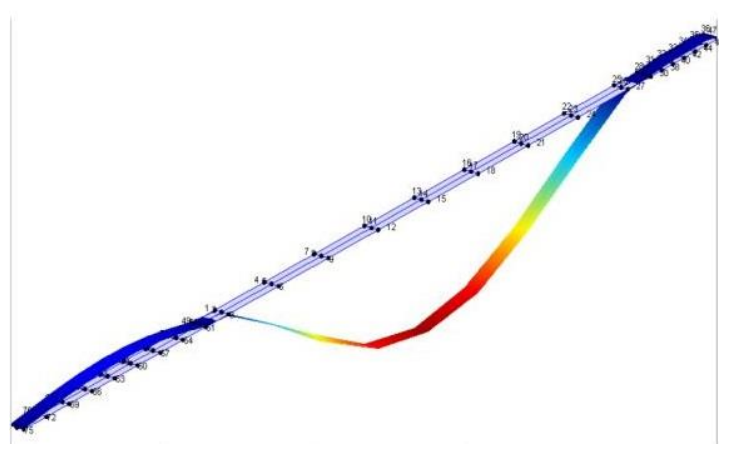

a) Primer modo

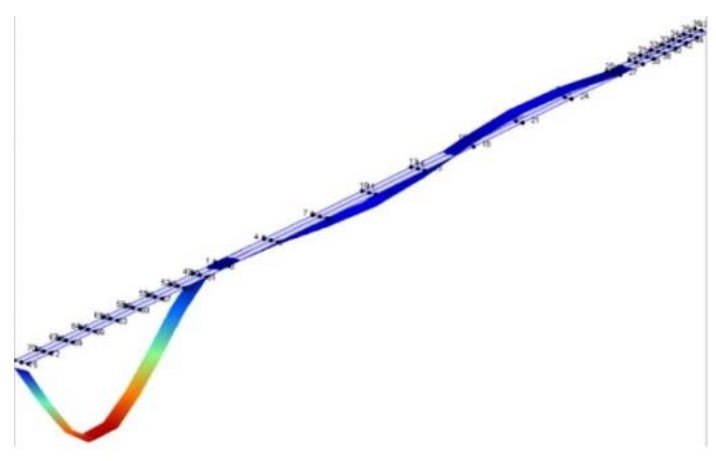

c) Tercer modo

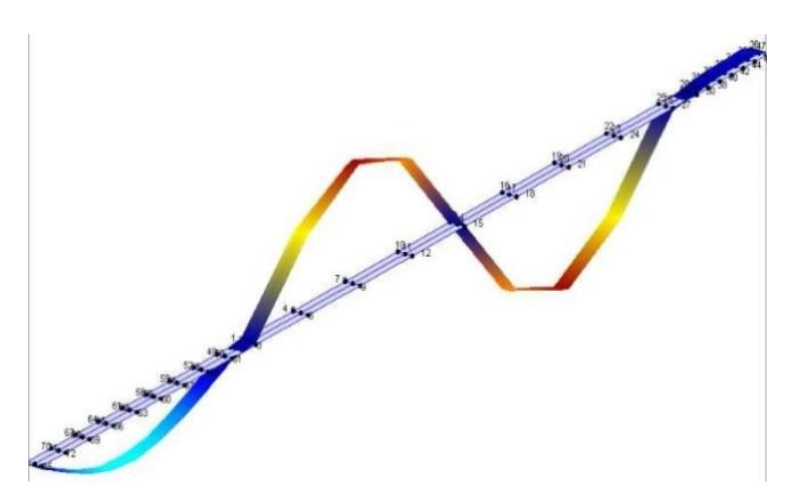

b) Segundo modo

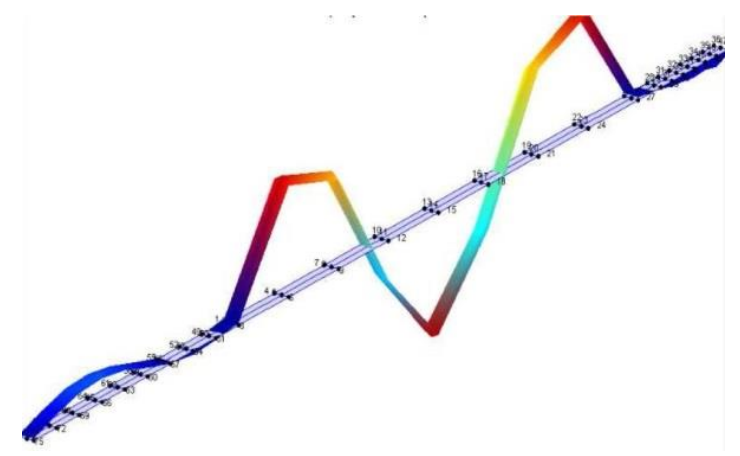

d) Cuarto modo

Figura 5-7. Primeros cuatro modos verticales de vibración de la pasarela obtenidos experimentalmente.

Debido a la mayor susceptibilidad del vano 2 de la pasarela, se realizó un EMA en éste identificando, entre otros, la masa modal vertical. Se realizaron asimismo en este mismo estudio [2] varias estimaciones de dicha masa modal empleando diferentes algoritmos, presentándose en la siguiente tabla los resultados obtenidos.

\begin{tabular}{cc}
\hline MÉTODO & MASA MODAL (kg) \\
\hline Programa OROS & 16983 \\
Algoritmo en frecuencia & 17950 \\
Algoritmo en el tiempo & 18060 \\
Ajuste de la curva FRF & 17900 \\
Aumento de la respuesta & 17500 \\
\hline
\end{tabular}

Tabla 5-2. Estimaciones de masa modal del tercer modo vertical.

Para el FEM Updating del modelo de la pasarela se emplearon todos los datos experimentales disponibles para los cuatro primeros modos verticales de vibración de la 
pasarela completa y que se han expuesto anteriormente, esto es, las frecuencias y las formas modales de dichos modos y la masa modal en el caso particular del $3^{\circ}$ modo de vibración de la pasarela completa.

Se empleó un esquema iterativo que nos permitió analizar y seleccionar aquellos parámetros físicos cuyo efecto era más relevante para que las frecuencias y las formas modales de nuestro modelo se aproximaran lo más posible a las obtenidas experimentalmente. En nuestro caso se comprobó que el tipo de apoyos (rigideces de los mismos) y la masa eran las variables que mayor influencia tenían en el ajuste una vez modelizada la geometría del tablero.

En el primer caso ha sido necesario, para obtener un ajuste adecuado, la modelización completa de las torres de celosía que sirven de apoyo central ya que la sustitución de éstas por simples apoyos, empotramientos y/o muelles (longitudinales y/o rotacionales) no ha dado buenos resultados. En el caso de la masa, ha sido la principal variable que ha afectado al ajuste, no sólo de la frecuencia y la forma modal sino también de su masa modal.

\subsubsection{Resultados}

Los resultados obtenidos con el modelo para los primeros cuatro modos son los que a continuación se reflejan:

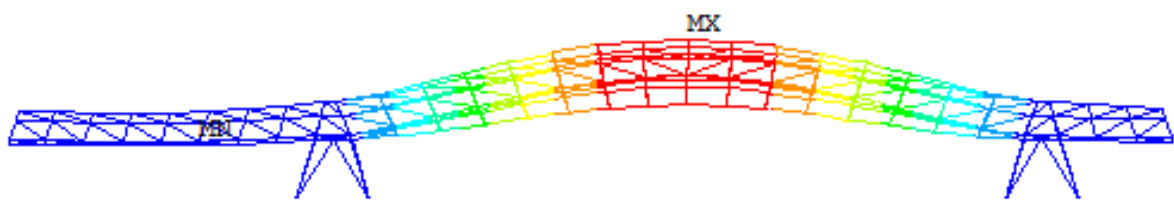

Figura 5-8. Primer modo vertical: $\mathrm{f}_{1}=1.224 \mathrm{~Hz}$ obtenido con el modelo en ANSYS.

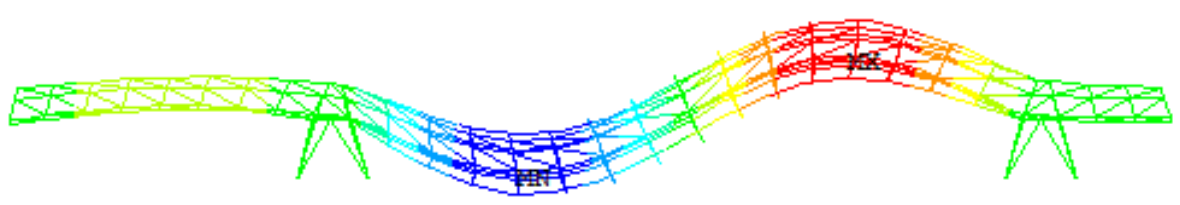

Figura 5-9. Segundo modo vertical: $f_{2}=2.842 \mathrm{~Hz}$ obtenido con el modelo en ANSYS. 


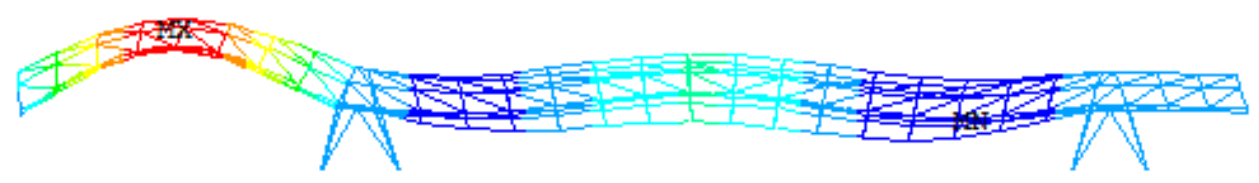

Figura 5-10. Tercer modo vertical: $f_{3}=3.662 \mathrm{~Hz}$ obtenido con el modelo en ANSYS.

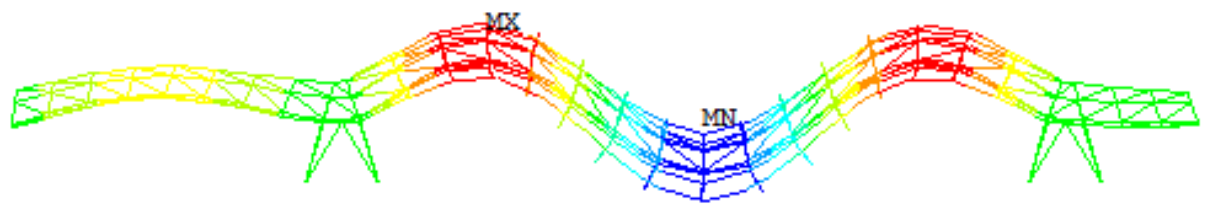

Figura 5-11. Cuarto modo vertical: $\mathrm{f}_{4}=4.914 \mathrm{~Hz}$ obtenido con el modelo en ANSYS.

Tal y como puede comprobarse las formas modales del modelo son equivalentes a los obtenidos experimentalmente en [2]. Por otra parte, la siguiente tabla comparativa muestra que en todos los casos se obtienen diferencias menores del $5 \%$ en las frecuencias lo que indica una buena correspondencia del modelo.

\begin{tabular}{|c|c|c|c|c|}
\hline Modo & $\begin{array}{l}\text { fMEF } \\
(\mathbf{H z})\end{array}$ & $\begin{array}{c}\mathbf{f}_{\mathrm{MEF}}-\mathbf{f}_{\mathrm{FDD}} \mid / \mathbf{f}_{\mathrm{MEF}} \\
(\%)\end{array}$ & $\begin{array}{c}\mathbf{f}_{\mathrm{MEF}}-\mathbf{f}_{\mathrm{EFDD}} \mid / \mathbf{f}_{\mathrm{MEF}} \\
(\%)\end{array}$ & $\begin{array}{c}\mathbf{f}_{\mathrm{MEF}}-\mathbf{f}_{\mathrm{CFDD}} \mid / \mathbf{f}_{\mathrm{MEF}} \\
(\%)\end{array}$ \\
\hline 1 & 1.224 & 1.72 & 1.55 & 2.70 \\
\hline 2 & 2.842 & 2.92 & 2.78 & 2.71 \\
\hline 3 & 3.662 & 3.99 & 4.04 & 4.01 \\
\hline 4 & 4.914 & 2.63 & 2.48 & 2.48 \\
\hline
\end{tabular}

Tabla 5-3. Comparativa entre las frecuencias obtenidas experimentalmente y las obtenidas mediante la modelización de la pasarela en ANSYS.

En el caso de la masa modal del primer modo del vano 2 se ajustó a un valor de $18057 \mathrm{~kg}$ que corresponde aproximadamente al valor máximo que se ha obtenido en las estimaciones experimentales. La diferencia en porcentaje con respecto al resto de las estimaciones no supera el $6 \%$. 


\subsection{Resultados obtenidos aplicando las metodologías propuestas}

Empleando los registros del EMA realizados en el vano 2 en [99, 100] se calcularon las masas modales y los modos normalizados asociados a cada modo de vibración de dicho vano mediante dos técnicas de escalado. Ambas parten de la información obtenida al aplicar el método SSI, consistiendo la primera de ellas en la resolución de un problema simétrico de valores propios y la segunda en el escalado de los modos de vibración a partir de la estimación de los residuos de la descomposición en fracciones parciales de la Función de Respuesta en Frecuencia (FRF) de las mediciones. Aplicando ambas técnicas se calcularon las masas modales y los valores de los modos normalizados respecto a la matriz de masa en los puntos $\mathrm{L} / 3, \mathrm{~L} / 2$ y $2 \mathrm{~L} / 3$ del vano 2 ( $\mathrm{L}=51 \mathrm{~m}$ ). Las metodologías se han validado previamente mediante un primer problema numérico pero suficientemente general (viga biapoyada) y posteriormente se han aplicado a un ejemplo general más complejo (viga continua de tres vanos) y a la estructura objeto de este estudio, presentando a continuación los valores de los parámetros logrados.

\subsubsection{Problema simétrico de valores propios}

Tal y como hemos indicado anteriormente la metodología consiste en identificar el sistema en base a un modelo de espacio de estados a partir de los datos de respuesta, después se determinan los factores de escala dados por la matriz diagonal $\mathbf{T}$ y los vectores propios $\psi$ y finalmente se estiman las matrices de masa, amortiguamiento y rigidez del modelo de segundo orden ( $\mathbf{M}, \mathbf{C}$ y $\mathbf{K}$ respectivamente) lo que nos permite calcular todos los parámetros modales.

\section{Resultados de la validación (viga biapoyada)}

El problema planteado consiste es una viga biapoyada de longitud $\mathrm{L}=10,4 \mathrm{~m}$, módulo de Young $\mathrm{E}=2.1 \cdot 10^{11} \mathrm{~Pa}$, densidad $\rho=7850 \mathrm{~kg} / \mathrm{m}^{3}$, inercia $\mathrm{I}_{\mathrm{z}}=604 \cdot 10^{-8} \mathrm{~m}^{4}$ y sección transversal de área $\mathrm{A}=53.8 \cdot 10^{-4} \mathrm{~m}^{2}$. Se supone un factor de amortiguamiento de $0.5 \%$ para cada uno de los modos de vibración de dicha viga. Se simula mediante herramienta matemática de manera que se obtienen las aceleraciones en las posiciones L/4, L/2 y $3 \mathrm{~L} / 4$ 
$\left(\mathrm{y}_{1}, \mathrm{y}_{2}\right.$ e $\mathrm{y}_{3}$ respectivamente) cuando se aplica una fuerza externa conocida igual a $f(t)=10^{2} \cdot \sin (2 \pi t)$ en la sección correspondiente a $\mathrm{L} / 4$.

A continuación se recogen los valores estimados de las frecuencias propias $\left(f_{i}\right)$, los factores de amortiguamiento $\left(\xi_{i}\right)$ y las masas generalizadas $\left(M_{i}\right)$ correspondientes a los tres primeros modos así como los mismos valores calculados analíticamente.

\begin{tabular}{ccccccc}
\hline & \multicolumn{3}{c}{ Estimado } & \multicolumn{3}{c}{ Analítico } \\
\cline { 2 - 7 } modo & $f_{i}(\mathrm{~Hz})$ & $\xi_{i}(\%)$ & $M_{i}(\mathrm{~kg})$ & $f_{i}(\mathrm{~Hz})$ & $\xi_{i}(\%)$ & $M_{i}(\mathrm{~kg})$ \\
\hline 1 & 2.52 & 0.5 & 219.7 & 2.52 & 0.5 & 219.6 \\
2 & 10.06 & 0.5 & 216.9 & 10.07 & 0.5 & 219.6 \\
3 & 22.61 & 0.5 & 194.1 & 22.65 & 0.5 & 219.6 \\
\hline
\end{tabular}

Tabla 5-4. Comparativa entre los parámetros modales estimados mediante el problema simétrico de valores propios y los obtenidos analíticamente (viga biapoyada).

Por último, se presentan los modos de vibración y los modos de vibración normalizados respecto a la matriz de masa estimados aplicando la metodología descrita y los calculados analíticamente.

\begin{tabular}{ccccccc}
\hline & \multicolumn{5}{c}{ Estimado } & \multicolumn{5}{c}{ Analítico } \\
\cline { 2 - 7 } modo & 1 & 2 & 3 & 1 & 2 & 3 \\
\hline $\mathrm{y}_{1}$ & 0.7071 & -1.0 & -0.707 & 0.7071 & -1.0 & -0.7071 \\
$\mathrm{y}_{2}$ & 1.0 & 0 & 1.0 & 1.0 & 0.0 & 1.0 \\
$\mathrm{y}_{3}$ & 0.7071 & 1.0 & -0.707 & 0.7071 & 1.0 & -0.7071
\end{tabular}

Tabla 5-5.- Comparativa entre los modos de vibración estimados mediante el problema simétrico de valores propios y los obtenidos analíticamente (viga biapoyada). 


\begin{tabular}{ccccccc}
\hline & \multicolumn{5}{c}{ Estimado } & \multicolumn{5}{c}{ Analítico } \\
\cline { 2 - 7 } modo & 1 & 2 & 3 & 1 & 2 & 3 \\
\hline $\mathrm{y}_{1}$ & 0.0477 & -0.0674 & -0.0506 & 0.0477 & -0.0675 & -0.0477 \\
$\mathrm{y}_{2}$ & 0.0675 & 0.0 & 0.0718 & 0.0675 & 0.0 & 0.0675 \\
$\mathrm{y}_{3}$ & 0.0477 & 0.0674 & -0.0506 & 0.0477 & 0.0675 & -0.0477 \\
\hline
\end{tabular}

Tabla 5-6.- Comparativa entre los modos de vibración normalizados estimados mediante el problema simétrico de valores propios y los obtenidos analíticamente (viga biapoyada).

Se observa en estas tablas que los resultados para el tercer modo de vibración son menos precisos, esto se debe a que el tipo de carga aplicada no es capaz de excitar el tercer modo.

\section{Resultados del ejemplo (viga continua de tres vanos)}

El ejemplo consiste en una viga continua de tres vanos tal y como muestra la siguiente figura.

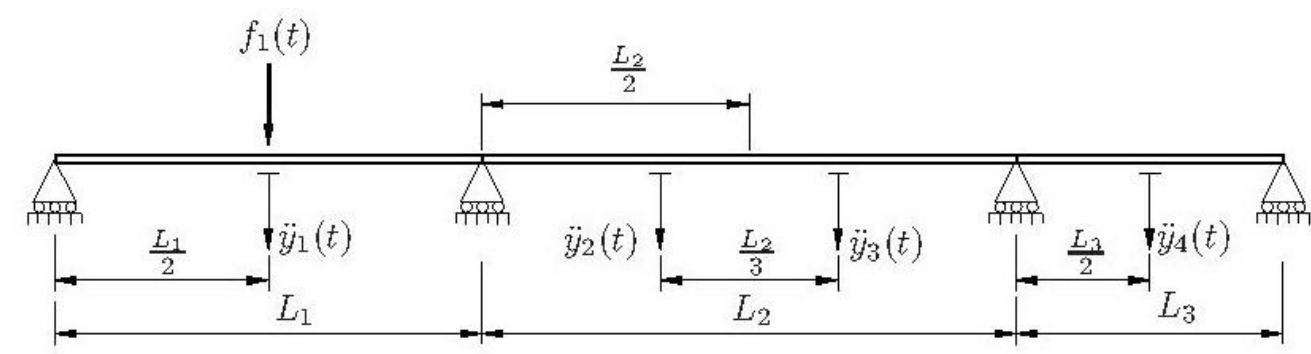

Figura 5-12. Viga continua que se emplea como ejemplo de aplicación de la metodología descrita.

La longitud de cada uno de los vanos es: $\mathrm{L}_{1}=8 \mathrm{~m}, \mathrm{~L}_{2}=10 \mathrm{~m}$ y $\mathrm{L}_{3}=5 \mathrm{~m}$. Las propiedades del material son: módulo de Young E=2.1·10 $11 \mathrm{~Pa}$, densidad $\rho=7850 \mathrm{~kg} / \mathrm{m}^{3}$. En cuanto a la sección transversal el área es igual a $A=53.8 \cdot 10^{-4} \mathrm{~m}^{2}$ y el momento de inercia es igual a $\mathrm{I}_{\mathrm{z}}=604 \cdot 10^{-8} \mathrm{~m}^{4}$.

De la resolución analítica del problema de análisis modal de dicha viga se obtienen los modos de vibración y frecuencias propias. Posteriormente se aplica una fuerza de tipo armónico igual a $f_{1}(t)=10^{2} \cdot \sin (2 \pi t)$ en la sección que indica la figura 5-12. Asimismo se asume un factor de amortiguamiento del $0.5 \%$ para cada uno de los modos de vibración y se calcula la respuesta dinámica de la estructura mediante el método de superposición modal (se consideran los seis primeros modos calculados anteriormente) con el objeto de generar los registros de aceleraciones en los puntos de la estructura indicados en la figura 
5-12 de un minuto de duración con un tiempo de muestreo $\mathrm{T}_{\mathrm{s}}=0.005 \mathrm{~s}$, para poder posteriormente emplear dichos registros, junto con el de la fuerza aplicada, como punto de partida en la aplicación de la metodología propuesta. La idea es contar con la misma información (registros de aceleraciones y de fuerza aplicada) que en el EMA según el esquema de la figura anterior.

Como ya hemos indicado anteriormente, se identifica el sistema y con el modelo obtenido en el espacio de estados se aplica la metodología expuesta y se obtienen las estimaciones que se resumen a continuación.

En la tabla siguiente se recogen los valores estimados de las frecuencias propias $\left(f_{i}\right)$, los factores de amortiguamiento $\left(\xi_{i}\right)$ y las masas generalizadas $\left(M_{i}\right)$ correspondientes a los cuatro primeros modos así como los mismos valores calculados analíticamente.

\begin{tabular}{ccccccc}
\hline & \multicolumn{3}{c}{ Estimado } & \multicolumn{3}{c}{ Analítico } \\
modo & $f_{i}(\mathrm{~Hz})$ & $\xi_{i}(\%)$ & $M_{i}(\mathrm{~kg})$ & $f_{i}(\mathrm{~Hz})$ & $\xi_{i}(\%)$ & $M_{i}(\mathrm{~kg})$ \\
\hline 1 & 3.75 & 0.50 & 146.6 & 3.75 & 0.50 & 146.5 \\
2 & 5.93 & 0.50 & 154.1 & 5.93 & 0.50 & 154.6 \\
3 & 11.69 & 0.49 & 116.3 & 11.69 & 0.50 & 124.9 \\
4 & 15.80 & 0.57 & 151.7 & 15.55 & 0.50 & 119.8 \\
\hline
\end{tabular}

Tabla 5-7. Comparativa entre los parámetros modales estimados mediante el problema simétrico de valores propios y los obtenidos analíticamente (viga continua de tres vanos).

Por último, se presentan en las siguientes tablas los modos de vibración y los modos de vibración normalizados respecto a la matriz de masa aplicando la metodología descrita y los calculados analíticamente. 


\begin{tabular}{ccccccccc}
\hline & \multicolumn{4}{c}{ Estimado } & \multicolumn{5}{c}{ Analítico } \\
\cline { 2 - 9 } modo & 1 & 2 & 3 & 4 & 1 & 2 & 3 & 4 \\
\hline $\mathrm{y}_{1}$ & -0.4783 & -0.8203 & 0.1317 & 0.0707 & -0.4783 & -0.8203 & 0.1315 & 0.0485 \\
$\mathrm{y}_{2}$ & 0.6467 & -0.2691 & 0.5576 & 0.3871 & 0.6467 & -0.2690 & 0.5578 & 0.3632 \\
$\mathrm{y}_{3}$ & 0.5797 & -0.4832 & -0.3897 & -0.5288 & 0.5797 & -0.4831 & -0.3902 & -0.5229 \\
$\mathrm{y}_{4}$ & -0.1297 & 0.1456 & 0.7210 & -0.7520 & -0.1297 & 0.1456 & 0.7206 & -0.7696 \\
\hline
\end{tabular}

Tabla 5-8.- Comparativa entre los modos de vibración estimados mediante el problema simétrico de valores propios y los obtenidos analíticamente (viga continua de tres vanos).

\begin{tabular}{ccccccccc}
\hline & \multicolumn{4}{c}{ Estimado } & \multicolumn{5}{c}{ Analítico } \\
\cline { 2 - 9 } modo & 1 & 2 & 3 & 4 & 1 & 2 & 3 & 4 \\
\hline $\mathrm{y}_{1}$ & -0.0395 & 0.0661 & 0.0123 & -0.0061 & -0.0395 & 0.0660 & 0.0118 & -0.0044 \\
$\mathrm{y}_{2}$ & 0.0534 & 0.0217 & 0.0521 & -0.0350 & 0.0534 & 0.0216 & 0.0499 & -0.0332 \\
$\mathrm{y}_{3}$ & 0.0479 & 0.0389 & -0.0364 & 0.0475 & 0.0479 & 0.0389 & -0.0349 & 0.0478 \\
$\mathrm{y}_{4}$ & -0.0107 & -0.0177 & 0.0672 & 0.0650 & -0.0107 & -0.0117 & 0.0645 & 0.0703 \\
\hline
\end{tabular}

Tabla 5-9.- Comparativa entre los modos de vibración normalizados estimados mediante el problema simétrico de valores propios y los obtenidos analíticamente (viga continua de tres vanos).

Se observa en estas tablas que los resultados estimados para el tercer y cuarto modo de vibración difieren ligeramente de los analíticos, que puede deberse a que su participación en la respuesta dinámica bajo la fuerza armónica propuesta es pequeña y por lo tanto la incertidumbre grande.

\section{Resultados de la pasarela}

En los EMA que se han realizado para la estimación de los parámetros modales del vano 2 de la pasarela del Museo de la Ciencia de Valladolid, se ha empleado una fuerza de excitación generada por un excitador electrodinámico colocado en el centro de dicho vano. Se ha utilizado un barrido continuo en frecuencia entre 2.5 y $4.5 \mathrm{~Hz}$ para excitar el primer modo de vibración. Se utilizaron 3 acelerómetros piezoeléctricos colocado en la línea central del vano 2 de la pasarela en las posiciones $(1 / 3,1 / 2,2 / 3) \cdot \mathrm{L}$, siendo $\mathrm{L}=51 \mathrm{~m}$. Se han llevado a cabo un total de nueve test.

A continuación se presentan los resultados obtenidos al aplicar esta metodología en la estructura objeto de estudio y que actualmente está en servicio [99]. 
En la siguiente tabla se recogen los valores medios estimados de las frecuencias propias $\left(f_{i}\right)$, los factores de amortiguamiento $\left(\xi_{i}\right)$ y las masas generalizadas $\left(M_{i}\right)$ correspondientes a los tres primeros modos así como el valor correspondiente de la desviación típica de cada magnitud $\left(\sigma_{f i}, \sigma_{\xi i}, \sigma_{M i}\right)$.

\begin{tabular}{ccccccc}
\hline modo & $f_{i}(\mathbf{H z})$ & $\sigma_{f i}(\mathbf{H z})$ & $\xi_{i}(\%)$ & $\sigma_{\xi i}(\%)$ & $M_{i}(\boldsymbol{k g})$ & $\sigma_{M i}(\mathbf{k g})$ \\
\hline 1 & 3,50 & 0,0088 & 0,64 & 0,3630 & 17605,0 & 1028,0 \\
2 & 7,32 & 0,1882 & 4,45 & 3,5991 & 13120,3 & 3656,1 \\
3 & 9,23 & 0,1073 & 1,43 & 1,1121 & 149759,9 & 45246,8 \\
\hline
\end{tabular}

Tabla 5-10. Valores medios y desviación típica de los parámetros modales estimados mediante el problema simétrico de valores propios.

El subíndice 1 corresponde a la posición L/3, el subíndice 2 a la posición L/2 y el subíndice 3 a la posición $2 \mathrm{~L} / 3$.

Se indican los tres primeros modos de vibración asociados a las masas generalizadas estimadas en la tabla siguiente mediante valor medio y desviación de cada una de las coordenadas.

\begin{tabular}{cccccccc}
\hline modo & $\mathbf{1}$ & $\mathbf{2}$ & $\mathbf{3}$ & modo & $\mathbf{1}$ & $\mathbf{2}$ & $\mathbf{3}$ \\
\hline $\mathrm{y}_{1}$ & 0,5146 & 1,0000 & $-0,9643$ & $\sigma_{\mathrm{y} 1}$ & 0,0067 & 0,0000 & 0,0801 \\
$\mathrm{y}_{2}$ & 1,0000 & 0,3114 & 0,8966 & $\sigma_{\mathrm{y} 2}$ & 0,0000 & 0,0636 & 0,1352 \\
$\mathrm{y}_{3}$ & 0,7636 & $-0,7001$ & $-0,5073$ & $\sigma_{\mathrm{y} 3}$ & 0,0067 & 0,1812 & 0,1376 \\
\hline
\end{tabular}

Tabla 5-11.- Modos de vibración estimados mediante el problema simétrico de valores propios.

Por último, se presentan los modos de vibración normalizados respecto a la matriz de masa que son los que se utilizan para simular la respuesta dinámica de la pasarela mediante el método de superposición modal. 


\begin{tabular}{cccccccc}
\hline modo & $\mathbf{1}$ & $\mathbf{2}$ & $\mathbf{3}$ & $\mathbf{m o d o}$ & $\mathbf{1}$ & $\mathbf{2}$ & $\mathbf{3}$ \\
\hline $\mathrm{y}_{1}$ & 0,0039 & 0,0090 & $-0,0026$ & $\sigma_{\mathrm{y} 1}$ & 0,0001 & 0,0013 & 0,0004 \\
$\mathrm{y}_{2}$ & 0,0075 & 0,0028 & 0,0024 & $\sigma_{\mathrm{y} 2}$ & 0,0002 & 0,0006 & 0,0004 \\
$\mathrm{y}_{3}$ & 0,0058 & $-0,0062$ & $-0,0013$ & $\sigma_{\mathrm{y} 3}$ & 0,0002 & 0,0014 & 0,0004 \\
\hline
\end{tabular}

Tabla 5-12.- Modos de vibración normalizados estimados mediante el problema simétrico de valores propios.

Aprovechando que el modelo de elementos finitos de la pasarela nos proporciona los modos de vibración normalizados respecto a la matriz de masa para el tercer modo de la pasarela completa, analizamos la correspondencia mediante el cálculo del MAC entre los valores obtenidos analítica y experimentalmente para dicho modo (que además es el que se ha obtenido con mayor precisión) y que reflejamos en la siguiente tabla comparativa:

\begin{tabular}{ccccc}
\hline $\begin{array}{c}\text { Técnica de obtención de los modos } \\
\text { normalizados }\end{array}$ & \multicolumn{3}{c}{ Coordenadas } & \multirow{2}{*}{ MAC } \\
\cline { 2 - 4 } Modelo ANSYS & 0.0069 & 0.0074 & 0.0055 & \\
\hline $\begin{array}{c}\text { Problema simétrico de valores } \\
\text { propios }\end{array}$ & 0.0039 & 0.0075 & 0.0058 & 0.938 \\
\hline
\end{tabular}

Tabla 5-13. Valores de MAC comparando los modos normalizados obtenidos numéricamente y los experimentales obtenidos mediante el problema simétrico de valores propios.

\subsubsection{Escalado de los modos de vibración a partir de la función de respuesta en frecuencia}

En este caso la metodología se resume en tres pasos: identificación del sistema en base a un modelo de espacio de estados a partir de los datos de respuesta, cálculo de los parámetros modales y finalmente escalado de los de vibración a partir de la estimación de los residuos de la descomposición en fracciones parciales de la FRF de las mediciones.

\section{Resultados de la validación (viga biapoyada)}

El problema planteado es el mismo que el descrito en el mismo subapartado del punto 5.2.1.

A continuación se recogen los valores estimados de las frecuencias propias $\left(f_{i}\right)$, los factores de amortiguamiento $\left(\xi_{i}\right)$ y las masas generalizadas $\left(M_{i}\right)$ correspondientes a los tres primeros modos así como los mismos valores calculados analíticamente. 


\begin{tabular}{ccccccc}
\hline & \multicolumn{3}{c}{ Estimado } & \multicolumn{3}{c}{ Analítico } \\
\cline { 2 - 7 } modo & $f_{i}(\mathrm{~Hz})$ & $\xi_{i}(\%)$ & $M_{i}(\mathrm{~kg})$ & $f_{i}(\mathrm{~Hz})$ & $\xi_{i}(\%)$ & $M_{i}(\mathrm{~kg})$ \\
\hline 1 & 2.52 & 0.5 & 219.5 & 2.52 & 0.5 & 219.6 \\
2 & 10.07 & 0.5 & 215.2 & 10.07 & 0.5 & 219.6 \\
3 & 22.65 & 0.5 & 186.2 & 22.65 & 0.5 & 219.6
\end{tabular}

Tabla 5-14. Comparativa entre los parámetros modales estimados mediante el escalado a partir de la FRF y los obtenidos analíticamente (viga biapoyada).

Por último, se presentan los modos de vibración y los modos de vibración normalizados respecto a la matriz de masa estimados aplicando la metodología descrita y los calculados analíticamente.

\begin{tabular}{ccccccc}
\hline & \multicolumn{5}{c}{ Estimado } & \multicolumn{5}{c}{ Analítico } \\
modo & 1 & 2 & 3 & 1 & 2 & 3 \\
\hline $\mathrm{y}_{1}$ & 0.7071 & -1.0 & -0.7071 & 0.7071 & -1.0 & -0.7071 \\
$\mathrm{y}_{2}$ & 1.0 & 0 & 1.0 & 1.0 & 0.0 & 1.0 \\
$\mathrm{y}_{3}$ & 0.7071 & 1.0 & -0.7071 & 0.7071 & 1.0 & -0.7071
\end{tabular}

Tabla 5-15.- Comparativa entre los modos de vibración estimados mediante el escalado a partir de la FRF y los obtenidos analíticamente (viga biapoyada).

\begin{tabular}{ccccccc}
\hline & \multicolumn{5}{c}{ Estimado } & \multicolumn{5}{c}{ Analítico } \\
\cline { 2 - 7 } modo & 1 & 2 & 3 & 1 & 2 & 3 \\
\hline $\mathrm{y}_{1}$ & 0.0477 & -0.0682 & -0.0518 & 0.0477 & -0.0675 & -0.0477 \\
$\mathrm{y}_{2}$ & 0.0675 & 0.0 & 0.0733 & 0.0675 & 0.0 & 0.0675 \\
$\mathrm{y}_{3}$ & 0.0477 & 0.0682 & -0.0518 & 0.0477 & 0.0675 & -0.0477 \\
\hline
\end{tabular}

Tabla 5-16.- Comparativa entre los modos de vibración normalizados estimados mediante el escalado a partir de la FRF y los obtenidos analíticamente (viga biapoyada).

Se observa en estas tablas que los resultados para el tercer modo de vibración son menos precisos, esto se debe a que el tipo de carga aplicada no es capaz de excitar el tercer modo.

\section{Resultados del ejemplo (viga continua de tres vanos)}

El ejemplo corresponde al descrito en el mismo subapartado del punto 5.2.1. 
A continuación se recogen los valores estimados de las frecuencias propias $\left(f_{i}\right)$, los factores de amortiguamiento $\left(\xi_{i}\right)$ y las masas generalizadas $\left(M_{i}\right)$ correspondientes a los cuatro primeros modos así como los mismos valores calculados analíticamente.

\begin{tabular}{ccccccc}
\hline & \multicolumn{3}{c}{ Estimado } & \multicolumn{3}{c}{ Analítico } \\
\cline { 2 - 7 } modo & $f_{i}(\mathrm{~Hz})$ & $\xi_{i}(\%)$ & $M_{i}(\mathrm{~kg})$ & $f_{i}(\mathrm{~Hz})$ & $\xi_{i}(\%)$ & $M_{i}(\mathrm{~kg})$ \\
\hline 1 & 3.75 & 0.5 & 350.5 & 3.75 & 0.5 & 350.2 \\
2 & 5.93 & 0.5 & 228.7 & 5.93 & 0.5 & 229.7 \\
3 & 11.69 & 0.5 & 220.8 & 11.69 & 0.5 & 240.4 \\
4 & 15.68 & 0.9 & 192.0 & 15.55 & 0.5 & 202.3 \\
\hline
\end{tabular}

Tabla 5-17. Comparativa entre los parámetros modales estimados mediante el escalado a partir de la FRF y los obtenidos analíticamente (viga continua de tres vanos).

Por último, se presentan en las siguientes tablas los modos de vibración y los modos de vibración normalizados respecto a la matriz de masa aplicando la metodología descrita y los calculados analíticamente.

\begin{tabular}{ccccccccc}
\hline & \multicolumn{3}{c}{ Estimado } & \multicolumn{5}{c}{ Analítico } \\
\cline { 2 - 9 } modo & 1 & 2 & 3 & 4 & 1 & 2 & 3 & 4 \\
\hline $\mathrm{y}_{1}$ & -0.7398 & 1.0 & 0.1820 & -0.0668 & -0.7395 & 1.0 & 0.1825 & -0.0630 \\
$\mathrm{y}_{2}$ & 1.0 & 0.3279 & 0.7774 & -0.4947 & 1.0 & 0.3279 & 0.7741 & -0.4719 \\
$\mathrm{y}_{3}$ & 0.8962 & 0.5889 & -0.5456 & 0.7022 & 0.8964 & 0.5889 & -0.5415 & 0.6794 \\
$\mathrm{y}_{4}$ & -0.2004 & -0.1775 & 1.0 & 1.0 & -0.2006 & -0.1775 & 1.0 & 1.0 \\
\hline
\end{tabular}

Tabla 5-18.- Comparativa entre los modos de vibración estimados mediante el escalado a partir de la FRF y los obtenidos analíticamente (viga continua de tres vanos). 


\begin{tabular}{ccccccccc}
\hline & \multicolumn{4}{c}{ Estimado } & \multicolumn{5}{c}{ Analítico } \\
\cline { 2 - 9 } modo & 1 & 2 & 3 & 4 & 1 & 2 & 3 & 4 \\
\hline $\mathrm{y}_{1}$ & -0.0395 & 0.0661 & 0.0122 & -0.0048 & -0.0395 & 0.0660 & 0.0118 & -0.0044 \\
$\mathrm{y}_{2}$ & 0.0534 & 0.0217 & 0.0523 & -0.0357 & 0.0534 & 0.0216 & 0.0499 & -0.0332 \\
$\mathrm{y}_{3}$ & 0.0479 & 0.0389 & -0.0367 & 0.0507 & 0.0479 & 0.0389 & -0.0349 & 0.0478 \\
$\mathrm{y}_{4}$ & -0.0107 & -0.0177 & 0.0673 & 0.0722 & -0.0107 & -0.0117 & 0.0645 & 0.0703 \\
\hline
\end{tabular}

Tabla 5-19.- Comparativa entre los modos de vibración normalizados estimados mediante el escalado a partir de la FRF y los obtenidos analíticamente (viga continua de tres vanos).

Se observa en estas tablas que los resultados estimados para el tercer y cuarto modo de vibración difieren ligeramente de los analíticos, que puede deberse a que su participación en la respuesta dinámica es pequeña y por lo tanto la incertidumbre mayor.

\section{Resultados de la pasarela}

Como en las secciones anteriores el caso estudiado corresponde al descrito en el mismo subapartado del punto 5.2.1.

A continuación se muestran los resultados obtenidos al aplicar esta metodología en la estructura objeto de estudio y que actualmente está en servicio [100].

En la siguiente tabla se recogen los valores medios estimados de las frecuencias propias $\left(f_{i}\right)$, los factores de amortiguamiento $\left(\xi_{i}\right)$ y las masas generalizadas $\left(M_{i}\right)$ correspondientes a los tres primeros modos así como el valor correspondiente de la desviación típica de cada magnitud $\left(\sigma_{f i}, \sigma_{\xi i}, \sigma_{M i}\right)$.

\begin{tabular}{ccccccc}
\hline modo & $f_{i}(\mathbf{H z})$ & $\sigma_{f i}(\mathbf{H z})$ & $\xi_{i}(\%)$ & $\sigma_{\xi i}(\%)$ & $M_{i}(\boldsymbol{k g})$ & $\sigma_{M i}(\boldsymbol{k g})$ \\
\hline 1 & 3.50 & 0.0088 & 0.64 & 0.3630 & 17814.9 & 952.9 \\
2 & 7.32 & 0.1882 & 4.45 & 3.5991 & 13705.9 & 8384.2 \\
3 & 9.23 & 0.1073 & 1.43 & 1.1121 & 155695.4 & 56434.7 \\
\hline
\end{tabular}

Tabla 5-20. Valores medios y desviación típica de los parámetros modales estimados mediante el escalado a partir de la FRF.

El subíndice 1 corresponde a la posición L/3, el subíndice 2 a la posición L/2 y el subíndice 3 a la posición 2L/3. 
Se muestran los tres primeros modos de vibración asociados a las masas generalizadas estimadas en la tabla siguiente mediante valor medio y desviación de cada una de las coordenadas.

\begin{tabular}{cccccccc}
\hline modo & $\mathbf{1}$ & $\mathbf{2}$ & $\mathbf{3}$ & modo & $\mathbf{1}$ & $\mathbf{2}$ & $\mathbf{3}$ \\
\hline $\mathrm{y}_{1}$ & 0.5142 & 1.0000 & -0.9267 & $\sigma_{\mathrm{y} 1}$ & 0.0050 & 0.0000 & 0.1739 \\
$\mathrm{y}_{2}$ & 1.0000 & 0.2384 & 0.8983 & $\sigma_{\mathrm{y} 2}$ & 0.0000 & 0.1821 & 0.1247 \\
$\mathrm{y}_{3}$ & 0.5951 & -0.6618 & -0.6207 & $\sigma_{\mathrm{y} 3}$ & 0.0082 & 0.1979 & 0.1445 \\
\hline
\end{tabular}

Tabla 5-21. Modos de vibración estimados mediante el escalado a partir de la FRF.

Por último, se presentan los modos de vibración normalizados respecto a la matriz de masa que son los que se utilizan para simular la respuesta dinámica de la pasarela mediante el método de superposición modal.

\begin{tabular}{cccccccc}
\hline modo & $\mathbf{1}$ & $\mathbf{2}$ & $\mathbf{3}$ & modo & $\mathbf{1}$ & $\mathbf{2}$ & $\mathbf{3}$ \\
\hline $\mathrm{y}_{1}$ & 0.0039 & 0.0096 & -0.0025 & $\sigma_{\mathrm{y} 1}$ & 0.0001 & 0.0029 & 0.0007 \\
$\mathrm{y}_{2}$ & 0.0075 & 0.0019 & 0.0023 & $\sigma_{\mathrm{y} 2}$ & 0.0002 & 0.0020 & 0.0004 \\
$\mathrm{y}_{3}$ & 0.0045 & -0.0060 & -0.0016 & $\sigma_{\mathrm{y} 3}$ & 0.0001 & 0.0019 & 0.0004 \\
\hline
\end{tabular}

Tabla 5-22. Modos de vibración normalizados estimados mediante el escalado a partir de la FRF.

Como en el apartado anterior, aprovechando que el modelo de elementos finitos de la pasarela nos proporciona los modos de vibración normalizados respecto a la matriz de masa para el tercer modo de la pasarela completa, analizamos la correspondencia entre los valores obtenidos analítica y experimentalmente mediante el cálculo del MAC para este modo (que además es el que se ha obtenido con mayor precisión) y que reflejamos en la siguiente tabla comparativa:

\begin{tabular}{|c|c|c|c|c|}
\hline \multirow{2}{*}{$\begin{array}{c}\text { Técnica de obtención de los modos } \\
\text { normalizados }\end{array}$} & \multicolumn{3}{|c|}{ Coordenadas } & \multirow{2}{*}{ MAC } \\
\hline & $\mathrm{L} / 3$ & $\mathrm{~L} / 2$ & $2 \mathrm{~L} / 3$ & \\
\hline Modelo ANSYS & 0.0069 & 0.0074 & 0.0055 & \\
\hline $\begin{array}{c}\text { Escalado a partir de la estimación } \\
\text { de los residuos de la } \\
\text { descomposición en fracciones } \\
\text { parciales de la Función de } \\
\text { Respuesta en Frecuencia (FRF) }\end{array}$ & 0.0039 & 0.0075 & 0.0045 & 0.944 \\
\hline
\end{tabular}

Tabla 5-23. Valores de MAC comparando los modos normalizados obtenidos numéricamente y los experimentales empleando el escalado a partir de la FRF. 



\section{CAPÍTULO 6. CONCLUSIONES}


Se ha desarrollado un modelo de elementos finitos de la Pasarela del Museo de la Ciencia de Valladolid mediante el software ANSYS para obtener las frecuencias y los modos de vibración de la misma. Para ello se han estudiado y posteriormente empleado los elementos mecánicos que mejor representan el comportamiento real de la estructura, en especial el pretensado.

Asimismo, durante el proceso de modelización se han analizado las variables que más influyen en el ajuste de las frecuencias analíticas con los resultados experimentales, seleccionando las más importantes y actuando sobre las mismas para su obtención. En concreto, para el caso estudiado, se ha constatado la importancia de la correcta simulación de las rigideces de los apoyos y de la masa.

Por otra parte, se ha estudiado la validez de la aplicación de dos técnicas: problema simétrico de valores propios y escalado de los modos de vibración a partir de la estimación de los residuos de la descomposición en fracciones parciales de la FRF, para la obtención de los principales parámetros modales -incluidas las masas modales o los modos normalizados respecto a la matriz de masa-de la pasarela. Éstas parten de la técnica de identificación en el subespacio estocástico, el método de identificación más utilizado actualmente, con una mínima información experimental respecto a la entrada y sin necesidad de modelos computacionales complejos. 


\subsection{Modelo de elementos finitos de la pasarela completa}

A la vista de los resultados obtenidos se puede llegar a la conclusión de que el modelo de elementos finitos representa de forma bastante precisa los parámetros modales (frecuencias y modos de vibración) de la estructura a pesar de la complejidad geométrica de la misma y considerando la multitud de factores -y errores inherentes a ellos- que intervienen tanto en la obtención de los resultados numéricos como los experimentales.

Durante el proceso de modelización y ajuste se ha corroborado la idea, ya mencionada, de que no existe ningún procedimiento general ni completamente fiable que pueda ser formulado para todos los casos. No obstante, el proceso de actualización del modelo mecánico nos ha permitido comprobar qué elementos tienen una mayor influencia en el ajuste de las frecuencias y los modos y cuáles apenas afectan. En este caso la solución ha dependido de los parámetros y constantes seleccionados - masa del vano 2 y rigideces de los apoyos intermedios- así como de la técnica empleada -método iterativo con uso de datos modales-.

El mayor interés de hacer modelados realistas y completos radica en que el análisis del proceso de modelización y ajuste puede realizarse sobre variables con significado físico (masa, pretensado, condiciones de apoyo...), que nos permitirá estudiar cómo las variaciones en dichos factores afectan al comportamiento global de la estructura. Todo el conocimiento generado puede ser aplicado al diseño de otras estructuras similares o a modificaciones del diseño original de la estructura estudiada. Por otra parte, en los modelados parciales se deben realizar un mayor número de hipótesis simplificativas que incrementan la incertidumbre en relación, entre otros, a la interpretación de los resultados obtenidos. 


\subsection{Métodos de escalado propuestos}

Analizados los resultados experimentales obtenidos aplicando las dos técnicas propuestas - problema simétrico de valores propios y escalado de los modos de vibración a partir de la estimación de los residuos de la descomposición en fracciones parciales de la FRF-, la primera conclusión que merece la pena reseñar, por la relevancia que tiene sobre el resto de las interpretaciones de los datos resultantes, es que sólo se pueden obtener con precisión los parámetros modales que corresponden a los modos de vibración que seamos capaces de excitar. Aunque lógica, esta conclusión ha de estar siempre presente tanto en la planificación de las pruebas como en el postprocesado de los registros. También se debe tener en cuenta que la incertidumbre en los resultados será mayor a medida que la participación en la respuesta dinámica de los modos sea menor.

Relativo a la precisión en la estimación de los modos de vibración normalizados respecto a la matriz de masa, se considera que es suficiente, teniendo en cuenta además la dificultad que tiene su obtención a partir de los registros experimentales, ya que en los métodos de identificación de sistemas no se tiene en ningún momento una expresión explícita de la matriz de masa. También se obtiene buena precisión en la determinación de las frecuencias propias, siendo menor en otras magnitudes como los factores de amortiguamiento, las masas generalizadas o los modos de vibración.

La principal ventaja que presentan estos métodos de escalado radica en que con una mínima información experimental respecto a la entrada (un solo canal coincidente con el correspondiente registro de salida) y tan sólo con las transformaciones matemáticas indicadas, se consigue determinar las masas modales excitables (o los modos normalizados respecto a la matriz de masa). 


\subsection{Líneas futuras}

La generación de un modelo mecánico de la Pasarela del Museo de la Ciencia nos ha permitido estudiar los diferentes factores que provocan la susceptibilidad a las vibraciones de la pasarela y nos permitirá contemplar diferentes alternativas de diseño y/o predecir su comportamiento ante diferentes tipos de solicitaciones durante las diferentes fases del proyecto. Todo ello contribuirá a una mejor compresión del comportamiento dinámico de este tipo de estructuras favoreciendo asimismo la proliferación del uso de este tipo de herramientas y así ayudar a los proyectistas y a las administraciones responsables de su diseño y mantenimiento. Asimismo se puede emplear el conocimiento generado en el presente estudio en las fases de diseño de otros casos similares, reduciendo de este modo las inversiones que serían necesarias implantar para considerar efectos dinámicos no deseados en etapas más tardías que habitualmente son mucho más elevadas. Estos análisis pueden abrir nuevas líneas de investigación que pueden ser recogidas en futuras publicaciones.

Con respecto a la validación de las metodologías de escalado propuestas, éstas nos permiten obtener todos los parámetros modales que nos permiten simular la respuesta de la pasarela y predecir su comportamiento dinámico frente a distintos tipos de solicitaciones durante su fase de servicio empleando modelos simplificados por superposición modal. También nos permiten el calibrado de dichos parámetros en las formulaciones de elementos finitos (FEM Updating), ya que son identificados directamente a partir de los datos experimentales. Asimismo se pueden emplear para obtener las masas modales de los modos que fueran necesarios mitigar con absorbedores pasivos de vibraciones (TMDs, del inglés Tuned Mass Dampers)... Todo ello complementaría las líneas de investigación planteadas en el párrafo anterior. 



\section{ANEXO. PUBLICACIONES}


"Estimación de parámetros modales de estructuras civiles a partir de la función de respuesta en frecuencia”

M. Cacho-Pérez, N. Frechilla y A. Lorenzana

Revista Internacional de Métodos Numéricos para Cálculo y Diseño en Ingenieria. http://dx.doi.org/10.1016/j.rimni.2016.02.001. Online publication date: 1-Jun-2016. 


\begin{tabular}{|c|c|c|}
\hline \multirow[t]{2}{*}{$\begin{array}{l}\text { G Model } \\
\text { RIMNI-184; No. of Pages } 7\end{array}$} & ARICLE IN PRESS & \\
\hline & Rev. int. métodos numér. cálc. diseño ing. 2016; $x x x(x x): x x x-x x x$ & \\
\hline s.t. & $\begin{array}{c}\text { Revista Internacional de Métodos Numéricos para } \\
\text { Cálculo y Diseño en Ingeniería }\end{array}$ & 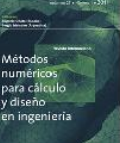 \\
\hline$\overline{\text { ELSEVIER }}$ & www.elsevier.es/rimni & \\
\hline
\end{tabular}

Estimación de parámetros modales de estructuras civiles a partir de la función de respuesta en frecuencia

M. Cacho-Perez ${ }^{*, 1}$, N. Frechilla ${ }^{2}$ y A. Lorenzana ${ }^{3}$

Escuela de Ingenierías Industriates, Universidad de Valladolid, Paseo del Cauce 59, 47011, Valladolid, Spain

\section{INFORMACIÓN DEL ARTÍCULO}

Historia del artículo

Recibido el 10 de septiembre de 2015 Aceptado el 19 de febrero de 2016 On-tine el $x \times x$

\section{Palabras clave:}

Pasarela peatona

Sisterna dinámico

\begin{abstract}
R E S U M E N
Este trabajo se centra en estimar los parámetros modales del sistema dinámico que constituye la pasarel peatonal del Museo de la Ciencia de la ciudad de Valladolid(Spain). El trabajo consiste no sólo en calcula frecuencias propias o naturales y factores de amortiguamiento asociados a cada uno de los modos estimados, como proporcionan muchos programas comerciales a partir de los registros de aceleraciones de ensayos OMA (Operational Modal Analysis) y/o EMA (Experimental Modal Analysis), sino que el objetivo de este articulo es calcular las masas generalizadas correspondientes a cada uno de los modos estimado de la estructura. Para ello, en primer lugar se obtiene una representación del sistema dinámico en el espacio de estados mediante la técnica SSI (Stochastic Subspace Identification), y en segundo lugar se escalan los modos de vibración a partir de la estimación de los residuos de la descomposición en fracciones parciales de la función de respuesta en frecuencia (FRF) de las mediciones. Con este método consiguen obtener buenas precisiones en los resultados, siempre que se consigan excitar los modos vibracín de los que queramos estimar los paŕmetros lo que nos permite simular la respuesta díńcica ve la de la estructura sin tener que recurrir a otros pros

(1) 2016 CIMNE (Universitat Politècnica de Catalunya). Publicado por Elsevier España, S.L.U. Este es un 2016 CIMNE (Universitat Politecnica de Catalunya). Publicado por Elsevier España, S.LU. Este es un
artículo Open Access bajo la CC BY-NC-ND licencia (http://creativecommons.org/licencias/by-nc-nd

\section{Estimation of modal parameters of civil structures from frequency response} function

A B S T R A C T

This paper focuses on estimating the modal parameters of the dynamic system that constitutes the pedestrian footbridge of the Science Museum of the city of Valladolid (Spain). The work consists not only o calculating natural frequencies and damping factors associated to each of the estimated modes, as many commercial software provide from acceleration measurements of tests OMA (Operational Modal Analysis) and/or EMA (Experimental modal Analysis), but the aim of this paper is to calculate the generalized masses corresponding to each of the estimated modes of the structure. For this purpose, firstly a representation of the dynamic system is obtained in the state space by SSI technique (Stochastic Subspac Identification), and secondly, vibration modes are scaled from the estimation of the residues of the

\section{Keywords:}

Footbridge

Dynamic system

* Autor para corres pondencia.

Correos electronicos: cacho@eii.uva.es 〈M. Cacho-Perez), noeliafrechilla@yahoo.es 〈N. Frechilla\}, ali@eii.uva.es 〈A. Lorenzana)

' Doctor Ingeniero Industrial, Profesor Ayudante Doctor.

${ }^{2}$ Investigador, Estudiante de doctorado en Ingeniería Industrial.

${ }^{3}$ Doctor Ingeniero Industrial, Profesor Titular.

http://dx.doi.org/10.1016/j.rimni.2016.02.001

0213-1315/0 2016 CIMNE 〈Universitat Politêcrica de Catalunya). Publicado por Elsevier España, S.L.U. Este es un artículo Open Access bajo la CCBY-NC-ND licencia 〈http:/ creativecommons.org/licencias;by-nc-nd/4.0/\}

Cômo citar este artículo: M. Cacho-Perez, et al. Estimaciôn de parámetros modales de estructuras civiles a partir de la función de respuesta en frecuencia, Rev. int. métodos numér. cálc. diseño ing. 2016. http://dx.doi.org/10.1016/j.rimni.2016.02.001 


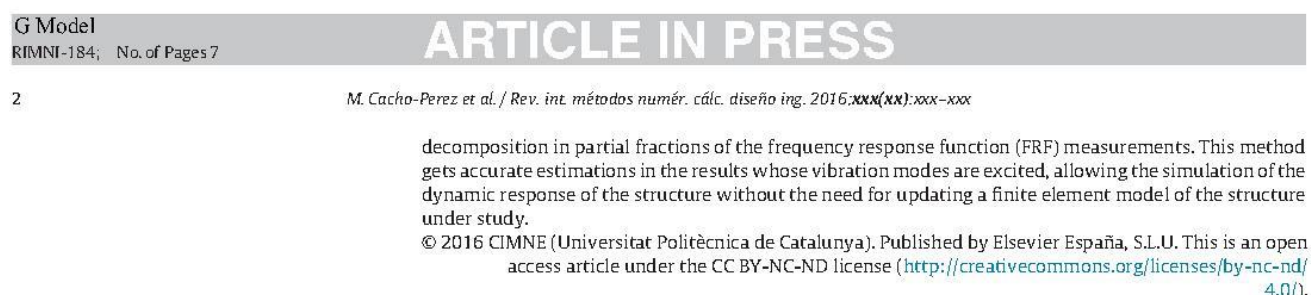

\section{Introducción}

Hoy en día existen estructuras típicas de proyectos e instalaciones de tipo industrial y de ingeniería civil, tales como chimeneas industriales, torres, edificios al tos, puentes, pasarelas o viaductos, que han sido diseñadas y ejecutadas bajo criterios distintos tos, que han sido diseñadas y ejecutadas bajo criterios distintos
a los actuales. Por otro lado, los nuevos materiales son cada vez a los actuales. Por otro lado, los nuevos materiales son cada vez
más resistentes y permiten el diseño y construcción de estructuras cada vez más esbeltas, complejas y ambiciosas, en las cuales los movimientos pueden ser apreciables. Estos aspectos, junto con las actuales demandas sociales relativas a la percepción de seguridad y confort, en algunos casos recogidas en las normativas y guías, suscitan la necesidad de desarrollar una metodología adecuada para el análisis y diseño asistido por ordenador de estructuras esbeltas, integrando técnicas experimentales que permitan una precisa identificación de los parámetros más relevantes que definen el comportamiento estructural (estático y dinámico).

En el caso concreto de las pasarelas peatonales, generalmente éstas son propensas a ser excitadas por las personas que las ocupan, apareciendo movimientos apreciables que incluso pueden llegar a ser molestos o, en algunos casos, inadecuados o peligrosos desde el punto de vista de la resistencia estructural [1].

Por otro lado, respecto a la metodología de cálculo dinámico, la técnica de análisis modal de estructuras consiste en la recolecciôn de datos, la identificación del sistema y, por último, la estimación de los parámetros modales. La etapa de identificación del sistema juega un papel crucial en los parámetros modales que se derivan, así como en el número de parámetros que se pueden determinar [2]. Los métodos de identificaciôn clásicos se basan en aplicar una excitaciôn al sistema y a continuaciôn registrar la correspondiente respuesta (Experimental Modal Analysis/EMA) [3]. Si los ensayos se realizan en condiciones de uso, en los que la magnitud de las fuerzas excitadoras es desconocida, el análisis se denomina Operational Modal Analysis/OMA y si los ensayos se llevan a cabo en condiciones de operaciôn aplicando una o varias fuerzas artificiales se denomina Operacional Modal Analysis with eXogenous inputs/OMAX.

Eperacional Modal Analysis with eXogenous inputs/OMAX.
En el caso de las estructuras civiles y mecánicas se usa en muchas ocasiones el OMA, ya que presentan ventajas económicas y operacionales, sin embargo, debido a que las fuerzas son desconocidas los modos de vibraciôn no pueden ser normalizados y el modelo resulta incompleto lo que no permite simular el sistema. Para solucionar este problema, se han desarrollado algunos métodos de escalado de los modos de vibraciôn que se basan en la actualizaciôn de un modelo de elementos finitos de la estructura empleando los parámetros modales estimados en el OMA [4,5]. También se han desarrollado soluciones basadas en la suposiciôn de que la matriz de masa de la estructura (o su inversa) es conocida [6] o modificando el comportamiento dinámico de la estructura variando la masa o la rigidez y realizando un análisis modal operacional tanto en la estructura original como en la modificada [7-13]. Estos métodos requieren un procedimiento experimental más extenso al emplear los parámetros modales de la estructura original y también de la modificada.

Otros métodos de escalado consisten en añadir sistemas de vibraciôn secundarios, midiendo y procesando la respuesta conjunta [14].
La identificación de los paráme tros en una formulación diferencial de primer orden también ha recibido considerable atención, pero como es bien sabido, si se parte de un espacio de estados modelo, y se trata de identificar los parámetros del modelo de segundo orden, cuestiones como la no unicidad de la solución comlica la resoluciôn drong tica la resolucion an desarrollado varias metodologías que resuelven el problema imponiendo diferentes restricciones en el número de sensores y actuadores empleados [22-26].

En el presente artículo se retoma el empleo de los resultados del EMA para obtener todos los parámetros modales necesarios para la simulación de una estructura civil, escalando los modos de vibracion a partir de crión en fracciones parciales de la función de respuesta en frecuencia (FRF) de las mediciones. Asimismo se aplica, una vez validada la metodología a través de dos casos teóricos, en una estructura actualmente en uso. La informaciôn de partida seobtiene aplicando uno de los métodos más utilizados en la actualidad, el SSI (Stochastic Subspace Identification), y se escoge el escalado de forma que el mayor coeficiente modal sea igual a 1 teniendo el que participa en cada modo de vibración] [27].

Por úl timo, indicar que el trabajo se ha organizado de la manera siguiente: en primer lugar, tras esta introducción, se presenta la metodología empleada. A continuación, se diserta sobre los resultados obtenidos y en el último apartado se presentan las principales conclusiones que se deducen de este trabajo.

\section{Metodología}

2.1. Función de transferencia

La función de transferencia de un sistema amortiguado de multiples grados de libertad puede ser formulada a partir de las ecuaciones del movimiento en términos de las matrices de masa, rigidez y amortiguamiento [28]:

$\mathbf{M} \ddot{\mathbf{u}}(t)+\mathbf{C} \dot{\mathbf{u}}(t)+\mathbf{K u}(t)=\mathbf{f}(t)$

donde $\mathbf{u}(t)$ indica el vector de desplazamientos nodales, con (?) y () que representan la primera y segunda derivada respecto al tiempo, el vector $\mathbf{f}(t)$ contiene las excitaciones externas que solicitan el sistema, las magnitudes $\mathbf{M}$, C y $\mathbf{K}$ son las matrices simétricas definidas positivas de masa, amortiguamiento y rigidez para un sistema de $N$ grados de libertad.

Aplicando la transformada de laplace a la ecuaciôn (1) y asumiendo condiciones iniciales nulas, resulta:

$\left(s^{2} \mathbf{M}+s \mathbf{C}+\mathbf{K}\right) \mathbf{U}(s)=\mathbf{F}(s)$

donde $\mathbf{U}(s)$ y $\mathbf{F}(s)$ son la transformada de Laplace de la respuesta en desplazamientos y de la fuerza, respectivamente. Se define la matriz dinámica del sistema, como:

$\mathbf{B}(\mathbf{s})=\left(\mathbf{s}^{2} \mathbf{M}+\mathbf{s} \mathbf{C}+\mathbf{K}\right)$ 
G Model RIMNI-184; No. of Pages 7 ARTICLE IN PRESS

M. Cacho-Perez et at. / Rev. int. métodos numér. cátc. diseño ing. 2016:xxx(xx):Xxx-XXx

Y a partir de la matriz dinámica se define la matriz función de transferencia:

$\mathbf{H}(s)=[\mathbf{B}(s)]^{-1}=\mathbf{U}(s) \cdot[\mathbf{F}(s)]^{-1}$

En general,

$H_{p q}(s)=\frac{U_{p}(s)}{F_{q}(s)}$

donde $p$ es el grado de libertad de salida y $q$ es el grado de libertad de entrada (orientaciôn y posición física).

Por lo tanto, la estimación de una columna de la matriz función de transferencia se lleva a cabo midiendo la respuesta dinámica en distintos puntos para un excitador ubicado en una posición fija.

\subsection{Residuos}

Cada uno de los términos de la matriz funciôn de transferenci se puede descomponer en fracciones parciales, tal y como se indic a continuaciôn:

$H_{p q}(s)=\sum_{r=1}^{N} \frac{A_{p q r}}{\left(s-\lambda_{r}\right)}+\frac{A_{p q r}^{*}}{\left(s-\lambda_{r}^{*}\right)}$

donde $A_{p q r}$ son los residuos asociados al polo $\lambda_{r}, y(\}^{*}$ indica el complejo conjugado. En términos de los valores modales del sistema se plejo conjugado. En tér

$H_{p q}(s)=\sum_{r=1}^{N} \frac{Q_{r} \psi_{p r} \psi_{q r}}{\left(s-\lambda_{r}\right)}+\frac{Q_{r}^{*} \psi_{p r}^{*} \psi_{q r}^{*}}{\left(s-\lambda_{r}^{*}\right)}$

siendo $Q_{r}$ el coeficiente de escalado del modo de vibración $\psi_{r}$.

2.3. Amortiguamiento proporcional (masa modal)

Para el caso de amortiguamiento proporcional, el cual incluye el caso sin amortiguamiento, se puede establecer una relación entre el residuo y la masa modal [29].

$M_{r}=\frac{1}{2 j \omega_{r} Q_{r}}$

$K_{r}=\frac{\omega_{r}}{2 j Q_{r}}$

donde $M_{r}$ y $K_{r}$ son la masa modal y la rigidez modal asociada al modo $\psi_{r}$, respectivamente. $Y$ siendo $\omega_{r}$ la frecuencia circular (rad/s) natural amortiguada (en el caso de que exista amortiguamiento). Además, se debe tener en cuenta que para los sistemas con amortiguamiento proporcional los vectores modales son siempre modos reales.

2.4. Amortiguamiento no proporcional (modal A)

El propósito del análisis modal es encontrar una transformaciôn de coordenadas que desacople las ecuaciones del movimiento. En un sistema con amortiguamiento no proporcional, las ecuaciones del movimiento se acoplan cuando se formulan en el espacio físico de dimensiôn $N$. Afortunadamente, las ecuaciones del movimiento pueden ser desacopladas cuando se formulan en el espacio de estados de dimensión $2 N$. Este úl timo se representa del siguiente modo [28]:

$\mathbf{A x}(t)+\mathbf{B x}(t)=\mathbf{f}_{s}(t)$

(10)

donde

$\mathbf{A}=\left[\begin{array}{ll}\mathbf{C} & \mathbf{M} \\ \mathbf{M} & \mathbf{0}\end{array}\right] \quad \mathbf{B}=\left[\begin{array}{ll}\mathbf{K} & \mathbf{0} \\ \mathbf{0} & -\mathbf{M}\end{array}\right]$

$$
\dot{\mathbf{x}}(t)=\left[\begin{array}{c}
\dot{\mathbf{u}}(t) \\
\ddot{\mathbf{u}}(t)
\end{array}\right] \quad \mathbf{x}(t)=\left[\begin{array}{l}
\mathbf{u}(t) \\
\dot{\mathbf{u}}(t)
\end{array}\right] \quad \mathbf{f}_{s}(t)=\left[\begin{array}{l}
\mathbf{I} \\
\mathbf{0}
\end{array}\right] \cdot \mathbf{f}(t)
$$

La ventaja de escribir las ecuaciones del movimiento de esta forma es que el problema de valores propios asociado es simétrico y puede plantearse en forma matricial como:

$\left[\begin{array}{ll}\mathbf{C} & \mathbf{M} \\ \mathbf{M} & \mathbf{0}\end{array}\right]\left[\begin{array}{l}\psi \\ \psi \boldsymbol{\Lambda}\end{array}\right] \Lambda=\left[\begin{array}{ll}-\mathbf{K} & \mathbf{0} \\ \mathbf{0} & \mathbf{M}\end{array}\right]\left[\begin{array}{l}\psi \\ \psi \boldsymbol{\Lambda}\end{array}\right]$

donde $\psi=\left[\psi_{1} \psi_{2} \ldots \psi_{2 N}\right]$ es la matriz que contiene los autovectores del problema complejo de valores propios siguiente:

$\left(\lambda_{i}^{2} \mathbf{M}+\lambda_{i} \mathbf{C}+\mathbf{K}\right) \psi_{i}=0$

y $\lambda_{i}=\zeta_{i} \pm j \omega_{i}$ los valores propios complejos (siendo $j=\sqrt{-1}$ ).

Al igual que en el caso de los sistemas no amortiguados, sonválidos un conjunto de relaciones de ortogonalidad para las matrices del sistema, en este caso A y

$\left[\begin{array}{l}\psi_{r} \\ \lambda_{r} \psi_{r}\end{array}\right]^{\top} \mathbf{A}\left[\begin{array}{l}\psi_{s} \\ \lambda_{s} \psi_{s}\end{array}\right]=0$

$\left[\begin{array}{l}\psi_{r} \\ \lambda_{r} \psi_{r}\end{array}\right]^{T} \mathbf{B}\left[\begin{array}{l}\psi_{s} \\ \lambda_{s} \psi_{s}\end{array}\right]=0$

Los términos modal $A$ y modal $B$ ahora se pueden definir de la siguiente manera:

$\left[\begin{array}{l}\psi_{r} \\ \lambda_{r} \psi_{r}\end{array}\right]^{T} \mathbf{A}\left[\begin{array}{l}\psi_{r} \\ \lambda_{r} \psi_{r}\end{array}\right]=M_{A_{r}}$

$\left[\begin{array}{l}\psi_{r} \\ \lambda_{r} \psi_{r}\end{array}\right]^{T} \mathbf{B}\left[\begin{array}{l}\psi_{r} \\ \lambda_{r} \psi_{r}\end{array}\right]=M_{B_{r}}$

Se debe tener en cuenta que estas cantidades tienen las mis mas propiedades que la masa modal y la rigidez modal para los casos sin amortiguamiento y con amortiguamiento proporcional. Siempre que los vectores modales sean complejos, las magnitudes modal $A$ y modal $B$ deben ser utilizadas para proporcionar el escalado modal.

\subsection{Identificación del sistema dinámico}

La técnica de identificaciôn consiste en la determinaciôn del modelo de primer orden del sistema dinámico. A partir de los datos de aceleración registrados en los sensores y de la fuerza aplicad mediante el actuador es posible identificar (mediante el método mediante el actuador es posible identificar (mediante el método
denominado Stochastic Subspace Identification, SSI) una represendenominado Stochastic Subspace Identification, SSI) una representaciôn en el esp

$$
\dot{\mathbf{x}}(t)=\mathrm{A}_{\mathrm{C}} \mathbf{X}(t)+\mathrm{B}_{\mathrm{C}} \mathbf{f}(t)
$$$$
\mathbf{y}(t)=\mathrm{C}_{\mathrm{C}} \mathbf{x}(t)+\mathrm{D}_{\mathrm{C}} \mathbf{f}(t)
$$

donde $\mathbf{A}_{C}, \mathbf{B}_{C}, \mathbf{C}_{C}$ y $\mathbf{D}_{C}$ son las matrices del sistema en tiempo continuo.

Una vez estimada la matriz $\mathbf{A}_{C}$ se procede a calcular sus valores propios $\lambda_{i}$ y los vectores propios asociados $\phi$. Y por último, se estiman los parámetros modales: frecuencias, factores de amorti guamiento y modos de vibración según las expresiones:

$\omega_{i}=\operatorname{Im}\left(\lambda_{i}\right)$

$$
\zeta_{i}=\frac{\operatorname{Re}\left(\lambda_{i}\right)}{\left|\lambda_{i}\right|}
$$

$\psi=\mathrm{C}_{\mathrm{C}} \cdot \phi$

Cómo citar este artículo: M. Cacho-Perez, et al. Estimaciôn de parámetros modales de estructuras civiles a partir de la funciôn de respuesta en frecuencia, Rev. int. métodos numér. cálc. diseño ing. 2016. http://dx.doi.org/10.1016/j.rimni.2016.02.001 


G Model
RIMNI-184; No. of Pages 7
4
M. Cacho-Perez et al./Rev. int métodos numér. cálc. diseño ing. 2015:xxx(xx):xxx-xxx

\section{Resultados numéricos}

Una vez expuesta la metodología, se procede a la validaciôn de la misma presentando un primer problema numérico pero suficientemente general (validaciôn \}, para después aplicar dicha metodología al análisis de un caso general más complejo (ejemplo) y de una estructura real actualmente en uso.

\subsection{Validación}

El problema que se plantea en este apartado es el de una viga biapoyada de longitud $L=10.4 \mathrm{~m}$, môdulo de Young $E=2.1 \cdot 10^{11} \mathrm{~Pa}$, densidad $\rho=7850.0 \mathrm{~kg} / \mathrm{m}^{3}$, inercia $I_{z}=604 \cdot 10^{-8} \mathrm{~m}^{4}$ y secciôn densidad $\rho=7850.0 \mathrm{~kg} / \mathrm{m}^{3}$, inercia $f_{z}=604 \cdot 10^{-8} \mathrm{~m}^{4}$ y sección
transversal de área $A=53.8 \cdot 10^{-4} \mathrm{~m}^{2}$. También se supone un factransversal de área $A=53.8 \cdot 10^{-4} \mathrm{~m}^{2}$. También se supone un fac-
tor de amortiguamiento de $0.5 \%$ para cada uno de los modos de tor de amortiguamiento de $0.5 \%$ para cada uno de los modos de
vibraciôn de dicha viga. Se simula mediante manipulador simbólico de manera que se obtienen las aceleraciones en las posiciones $(1 / 4$, $1 / 2,3 / 4\} \cdot L$ (sensores 1,2 y 3 , respectivamente) cuando se aplica una fuerza externa conocida $\left(f_{1}(t)=10^{2} \cdot \sin (2 \pi t)\right\}$ en la misma sección donde se ubica el sensor 1 (para el grado de libertad 1 .

A continuación, se sigue la metodología de este trabajo: primero se identifica el sistema en base a un modelo de espacio de estados a partir de los datos de respuesta, en segundo lugar se calculan todos los parámetros modales y, por úl timo, se escalan los modos de vibración a partir de la estimaciôn de los residuos de la descomposiciôn en fracciones parciales de la funciôn de respuesta en frecuencia (FRF) de las mediciones.

En la tabla 1 se recogen los resultados estimados de los parámetros modales: frecuencias propias $\left(f_{i}\right)$, factores de amortiguamiento $\left(\zeta_{i}\right)$, modal A $\left(M_{A_{i}}\right)$ y masas generalizadas $\left(M_{i}\right)$ asociados a los tres primeros modos de vibraciôn de la viga, así como, también se indica la sol ución analítica correspondiente.

En la tabla 2 se indican los tres primeros modos de vibraciôn estimados asociados al modal A $\left(M_{A_{i}}\right)$ y masa generalizada $\left(M_{i}\right)$ de la tabla 1.

La tabla 3 muestra los modos normalizados respecto de la matriz de masa de la viga biapoyada correspondientes a los tres primeros modos de vibraciôn. Se observa, al igual que en la tabla 1, que los resultados para el tercer modo de vibración son poco precisos, esto

Tabla 1

Viga biapoyada. Parámetros modales

\begin{tabular}{|c|c|c|c|c|c|c|c|c|}
\hline \multirow[b]{2}{*}{ modo } & \multicolumn{4}{|c|}{ Estimado } & \multicolumn{4}{|c|}{ Analítico } \\
\hline & $\overline{f_{i}\langle\mathrm{~Hz}\}}$ & $\zeta_{i}(\%)$ & $M_{A_{1}}$ & $M_{i}\langle\mathbf{k g}\}$ & $\overline{f_{i}\langle\mathrm{~Hz}\}}$ & $\zeta_{i}(\%)$ & $M_{A_{1}}$ & $M_{i}(\mathbf{k g})$ \\
\hline 1 & 2.52 & 0.5 & $6943.0 j$ & 219.5 & 2.52 & 0.5 & $6945.8 j$ & 219.6 \\
\hline 2 & 10.07 & 0.5 & $27221.0 j$ & 215.2 & 10.07 & 0.5 & $27783.1 \mathrm{j}$ & 219.6 \\
\hline 3 & 22.65 & 0.5 & $52997.0 j$ & 186.2 & 22.65 & 0.5 & $62511.9 j$ & 219.6 \\
\hline
\end{tabular}

Tabla 2

Viga biapoyada. Modos de vibración

\begin{tabular}{|c|c|c|c|c|c|c|}
\hline \multirow[b]{2}{*}{ modo } & \multicolumn{3}{|c|}{ Estimado } & \multicolumn{3}{|c|}{ Analítico } \\
\hline & 1 & 2 & 3 & 1 & 2 & 3 \\
\hline & 0.7071 & -1.0 & -0.7071 & 0.7071 & -1.0 & -0.7071 \\
\hline$y_{2}$ & 1.0 & 0.0 & 1.0 & 1.0 & 0.0 & 1.0 \\
\hline
\end{tabular}

Tabla 3

Viga biapoyada. Modos de vibración normalizados

\begin{tabular}{|c|c|c|c|c|c|c|}
\hline \multirow[b]{2}{*}{ modo } & \multicolumn{3}{|c|}{ Estimado } & \multicolumn{3}{|c|}{ Analítico } \\
\hline & 1 & 2 & 3 & 1 & 2 & 3 \\
\hline$y_{\mathrm{t}}$ & 0.0477 & -0.0682 & -0.0518 & 0.0477 & -0.0675 & -0.0477 \\
\hline$y_{2}$ & 0.0675 & 0.0 & 0.0733 & 0.0675 & 0.0 & 0.0675 \\
\hline$y_{3}$ & 0.0477 & 0.0682 & -0.0518 & 0.0477 & 0.0675 & -0.0477 \\
\hline
\end{tabular}

se debe a que el tipo de carga aplicada en el ejemplo no es capaz de excitar el tercer modo por lo que resultaría conveniente hacer un barrido en frecuencias.

3.2. Ejemplo

A continuaciôn se va a mostrar la metodología de este tra bajo para identificar el modelo modal de una viga continua de tres vanos, indicada en la figura 1. La longitud de cada uno de los vanos es: $L_{1}=8 \mathrm{~m}, L_{2}=10 \mathrm{~m}$ y $L_{3}=5 \mathrm{~m}$. Las propiedades de material y de la sección son respectivamente: módulo de Youn $E=2.1 .10^{11} \mathrm{~Pa}$ densidad $\rho=7850 \mathrm{~kg} / \mathrm{m}^{3}$, ́rea de la sección transversal $A=53.8 \cdot 10^{-4}$ De la resolución analítica del problema de análisis modal de dicha viga se obtienen los modos de vibración y frecuencias propias que se muestran a continuaciôn en la figura 2 . A continuaciôn, se aplica una fuerza de tipo armónico $\left(f_{1}(t)=10^{2} \cdot \sin (2 \pi t)\right\}$ en la secciôn que indica la figura 1 , así mismo, se asume de nuevo un factor de amortiguaniento $5=0.5 \%$ para cada uno de los modos de vibracion y se calcula la ción y se calcula la respuesta dinanica de la estructura mediante el método de superposición modal (se consideran los seis primeros modos calculados anteriormente) con el objeto de generar los registros de aceleraciones en los puntos de la estructura indicados en la figura 1 de un minuto de duración con un tiempo de muestreo $T_{S}=0.005 \mathrm{~s}$, para emplear dichos registros, junto con el de la fuerza aplicada, como punto de partida en la aplicación de la metodología objeto de este trabajo.

Es decir, la idea es suponer que solamente contamos con la información recogida en los registros de aceleraciones y el registro de fuerza aplicada, según el esquema de la figura 1. Se identifica el sistema, y con el modelo obtenido en el espacio de estados se aplica la metodología expuesta y se obtienen las estimaciones que se resumen a continuaciôn.

En la tabla 4 se comparan los resultados obtenidos para las frecuencias propias, factores de amortiguamiento, el parámetro modal A y la masa modal asociados a los cuatro primeros modos de vibraciôn de la estructura aplicando la metodología objeto del presente artículo (que se presentan bajo el título "Estimado") con la soluciôn obtenida resolviendo el problema analíticamente (que se presentan bajo el título "Analítico").

En la tabla 5 se muestran los cuatro primeros modos de vibración de la viga continua aplicando la metodología estudiada y los resultados obtenidos analíticamente.

La tabla 6 presenta los cuatro modos de vibración anteriormente reflejados en la tabla anterior pero ahora normalizados respecto a la matriz de masa de la viga continua.

Se observa en los resultados de los modos de vibración tercero y cuarto una menor precisión debido a que su participaciôn en la respuesta dinámica es pequeña y por lo tanto la incertidumbre es mayor.

3.3. Aplicación a un caso real

En esta secciôn se describe la identificaciôn de los parámetros modales de la pasarela peatonal del Museo de la Ciencia (PMC) de la ciudad de Valladolid. Esta identificaciôn consistió en un análisis Tabla 4
Ejemplo. Viga continua. Parámetros modales

\begin{tabular}{|c|c|c|c|c|c|c|c|c|}
\hline \multirow[b]{2}{*}{ modo } & \multicolumn{4}{|c|}{ Estimado } & \multicolumn{4}{|c|}{ Analítico } \\
\hline & $f_{i}\langle\mathrm{~Hz}\}$ & $\zeta\{\%\}$ & $M_{A_{1}}$ & $M_{i}\langle\mathbf{k g}\rangle$ & $f_{i}\langle\mathrm{~Hz}\rangle$ & $\zeta_{i}(\%)$ & $M_{A_{l}}$ & $M_{i}(\mathbf{k g})$ \\
\hline 1 & 3.7 & 0.5 & 1653 & 350.5 & 3.75 & 0.5 & $16526.2 j$ & 350.2 \\
\hline 2 & & 0.5 & & & & 0.5 & & 22 \\
\hline & 11. & 0.5 & & $22 \mathrm{C}$ & 11.69 & 0.5 & & 240.4 \\
\hline 4 & 15.68 & 0.9 & $0 j$ & 192.0 & 15.55 & 0.5 & $39528.1 \mathrm{j}$ & 202.3 \\
\hline
\end{tabular}


G Model

RIMNI-184; No. of Pages 7

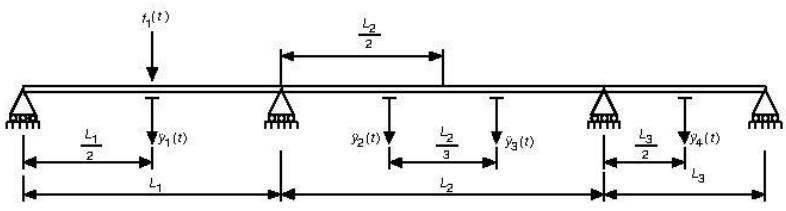

Figura 1. Viga continua.
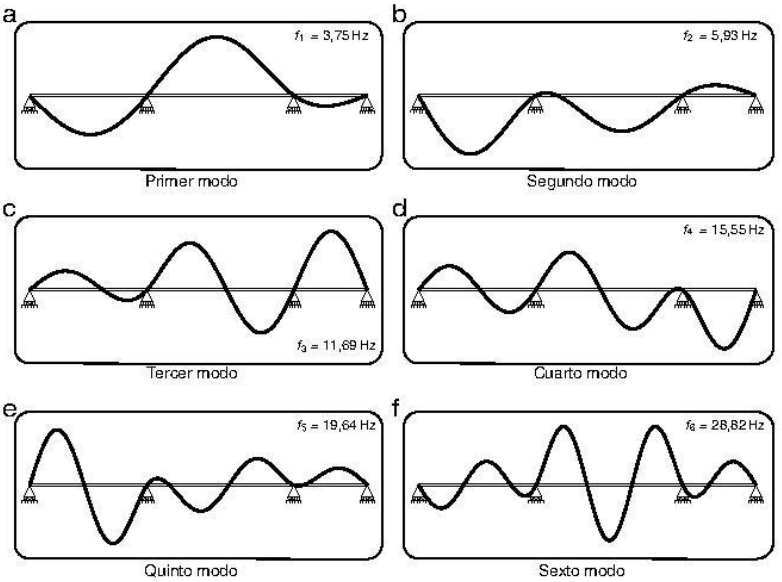

Figura 2. Viga continua. Modos de vibración.

modal experimental (EMA) del vano de la estructura más sensible a las vibraciones inducidas por el tránsito de peatones.

3.3.1. Descripción de la estructuro

La estructura estudiada, situada en Valladolid, es una pasarela peatonal sobre el río Pisuerga que une el Museo de la Ciencia con el centro de la ciudad. Este puente, construido en el año 2004, es una estructura de 234 metros compuesta de cuatrovanos: tres formados por barras metálicas tubulares y uno de hormigón. Los tres vanos metálicos tienen secciôn hexagonal, el vano principal (vano 3 en la figura 3 ) tiene 111 metros y cuenta con un sistema de tendones externos postensados, el segundo vano(vano 2 ) tiene 51 metros

Tabla 5

Ejemplo. Viga continua. Modos de vibración

\begin{tabular}{|c|c|c|c|c|c|c|c|c|}
\hline \multirow[b]{2}{*}{ modo } & \multicolumn{4}{|c|}{ Estimado } & \multicolumn{4}{|c|}{ Analítico } \\
\hline & 1 & 2 & 3 & 4 & 1 & 2 & 3 & 4 \\
\hline$y_{1}$ & -0.7398 & 1.0 & 0.1820 & -0.0668 & -0.7395 & 1.0 & 0.1825 & -0.0630 \\
\hline$y_{2}$ & 1.0 & 0.3279 & 0.7774 & -0.4947 & 1.0 & 0.3279 & 0.7741 & -0.4719 \\
\hline$y_{3}$ & 0.8962 & 0.5889 & -0.5456 & 0.7022 & 0.8964 & 0.5889 & -0.5415 & 0.6794 \\
\hline$y_{4}$ & -0.2004 & -0.1775 & 1.0 & 1.0 & -0.2006 & -0.1775 & 1.0 & 1.0 \\
\hline
\end{tabular}

Tabla 6

Ejemplo. Viga continua. Modos de vibración normalizados

\begin{tabular}{|c|c|c|c|c|c|c|c|c|}
\hline \multirow[b]{2}{*}{ modo } & \multicolumn{4}{|c|}{ Estimado } & \multicolumn{4}{|c|}{ Analítico } \\
\hline & 1 & 2 & 3 & 4 & 1 & 2 & 3 & 4 \\
\hline$y_{\mathrm{l}}$ & -0.0395 & 0.0661 & 0.0122 & -0.0048 & -0.0395 & 0.0660 & 0.0118 & -0.0044 \\
\hline$y_{2}$ & 0.0534 & 0.0217 & 0.0523 & -0.0357 & 0.0534 & 0.0216 & 0.0499 & -0.0332 \\
\hline$y_{3}$ & 0.0479 & 0.0389 & -0.0367 & 0.0507 & 0.0479 & 0.0389 & -0.0349 & 0.0478 \\
\hline$y_{4}$ & -0.0107 & -0.0117 & 0.0673 & 0.0722 & -0.0107 & -0.0117 & 0.0645 & 0.0703 \\
\hline
\end{tabular}

Cômo citar este artículo: M. Cacho-Perez, et al. Estimaciôn de parámetros modales de estructuras civiles a partir de la funciôn de respuesta en frecuencia, Rev. int. métodos numér. cálc. diseño ing. 2016. http://dx.doi.org/10.1016/j.rimni.2016.02.001 


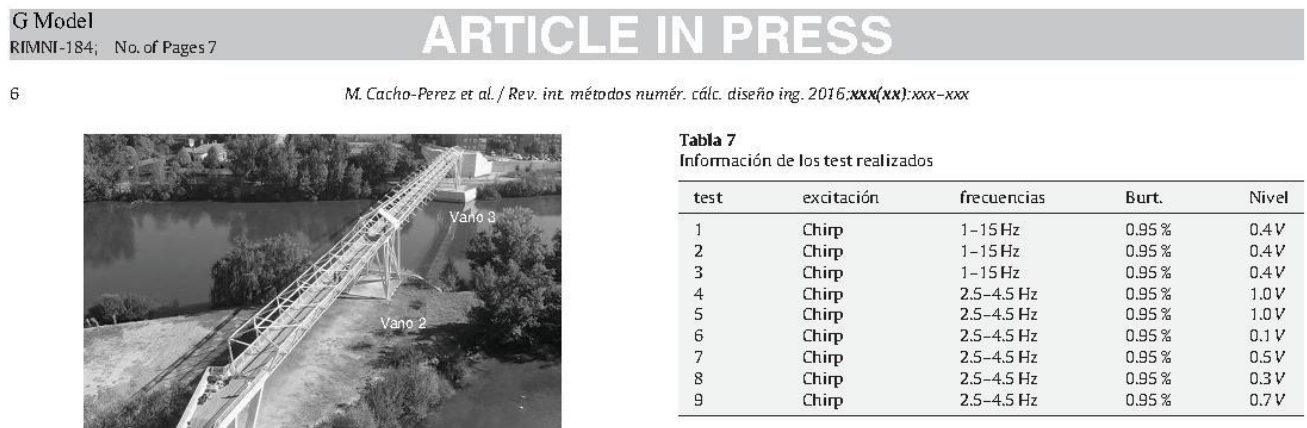

La tabla 8 recoge los valores medios estimados de las frecuencias propias $\left(\bar{f}_{i}\right)$, los factores de amortiguamiento $\left(\bar{\zeta}_{i}\right)$ y las masas generalizadas $\left(\bar{M}_{i}\right)$ correspondientes a los tres primeros modos de vibración del vano 2 de la pasarela. Esta misma tabla incluye el valor correspondiente de la desviaciôn típica o estándar de cada una de dichas magnitudes: frecuencias $\left(\sigma_{f_{f}}\right.$, factores de amortiguamiento $\left(\sigma_{\zeta_{i}}\right)$ y masas generalizadas $\left(\sigma_{M_{i}}\right)$, respectivamente. Se comprueba $\left(\sigma_{\zeta_{i}}\right\}$ y masas generalizadas $\left(\sigma_{M_{i}}\right)$, respectivamente. Se comprueba
que el método de identificación del sistema dinámico es preciso para estimar frecuencias de vibración, pero respecto a los resultados de amortiguamiento y masas generalizadas hay una dispersiôn importante.

A continuación, en la tabla 9 se indican los tres primeros modos de vibración asociados a las masas generalizadas estimadas en la tabla anterior (tabla 8), mediante valor medio y desviación de cada una de las coordenadas. Se observa falta de precisión en la estimación de los modos 2 y 3.

Y por último, se indica en la tabla 10 los modos de vibraciôn normalizados respecto de la matriz de masa, en base a valores medios y desviación típica de las coordenadas medidas. Destaca que se obtiene una precisión suficiente desde el punto de vista práctico con losvalores estimadosen dichatabla Porlotanto, se recomienda tralosvalores estimadosendichatabla Por panto se recomienda bajar siem masa para simular la respuesta dinámica de la estructura, por ejemplo, aplicando el conocido método de análisis por superposiciôn modal de un número fini to y pequeño de modos de vibración. y multiplicándola por su magnitud $(30.4 \mathrm{~kg})$. Se utilizó un barrido continuo en frecuencia entre 2.5 y $4.5 \mathrm{~Hz}$ para excitar el primer modo de vibraciôn. Se utilizaron 3 acelerómetros piezoeléctricos colocados en la línea central de la pasarela en las posiciones $(1 / 3$, $1 / 2,2 / 3\} \cdot L$ (siendo $L=51 \mathrm{~m}$. Este método tiene la ventaja de conocer la excitaciôn y el inconveniente de que se necesita un excitador capaz de hacer vibrar la estructura.

\subsubsection{Resultados experimentales}

Se llevaron a cabo un total de nueve test, en todos ellos el shaker si situôen la sección central del vano de estudio. Los datos concretos de cada test se indican en la tabla 7.

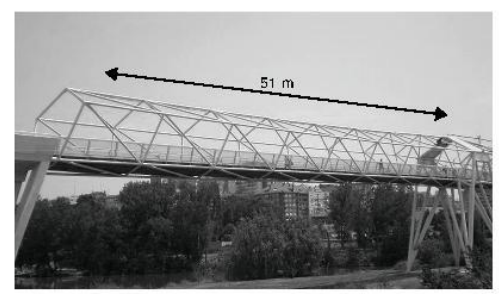

Figura 4. Vista del Vano 2.

Tabla 8

\begin{tabular}{|c|c|c|c|c|c|c|}
\hline modo & $\bar{f}_{i}\langle\mathrm{~Hz}\}$ & $\sigma_{h_{1}}\{\mathrm{~Hz}\}$ & $\bar{\zeta}_{i}(\%)$ & $\sigma_{\zeta_{1}\{\%}$ & $\bar{M}_{\mathrm{i}}\{\mathbf{k g}\}$ & $\sigma_{M_{1}}\{\mathbf{k g}\}$ \\
\hline 1 & 3.50 & 0.0088 & 0.64 & 0.3630 & 17814.9 & 952.9 \\
\hline 2 & $\begin{array}{l}7.32 \\
9.23\end{array}$ & 0.1882 & $\begin{array}{l}4.45 \\
1.43\end{array}$ & $\begin{array}{l}3.5991 \\
11121\end{array}$ & $\begin{array}{r}13705.9 \\
1556954\end{array}$ & $\begin{array}{r}8384.2 \\
56434.7\end{array}$ \\
\hline
\end{tabular}

Tabla 9

\begin{tabular}{|c|c|c|c|c|c|c|c|}
\hline modo & 1 & 2 & 3 & modo & 1 & 2 & 3 \\
\hline $\begin{array}{l}\bar{y}_{1} \\
\overline{y_{2}}\end{array}$ & $\begin{array}{l}0.5142 \\
1.0000\end{array}$ & $\begin{array}{l}1.0000 \\
0.2384\end{array}$ & $\begin{array}{r}-0.9267 \\
0.8983\end{array}$ & $\begin{array}{l}\sigma_{y_{1}} \\
\sigma_{y_{2}}\end{array}$ & $\begin{array}{l}0.0050 \\
0.0000\end{array}$ & $\begin{array}{l}0.0000 \\
0.1821\end{array}$ & $\begin{array}{l}0.1739 \\
0.1247\end{array}$ \\
\hline$\frac{y_{2}^{2}}{y_{3}}$ & 0.5951 & -0.6618 & -0.6207 & $\begin{array}{l}\sigma_{y_{y_{3}}} \\
\sigma_{1}\end{array}$ & 0.0082 & 0.1979 & 0.1445 \\
\hline
\end{tabular}

Tabla 10

\begin{tabular}{|c|c|c|c|c|c|c|c|}
\hline modo & 1 & 2 & 3 & modo & 1 & 2 & 3 \\
\hline $\begin{array}{l}\bar{y}_{1} \\
\bar{y}_{2}\end{array}$ & $\begin{array}{l}0.0039 \\
0.0075\end{array}$ & $\begin{array}{l}0.0096 \\
0.0019\end{array}$ & $\begin{array}{r}-0.0025 \\
0.0023\end{array}$ & $\begin{array}{l}\sigma_{y_{1}} \\
\sigma_{y_{2}}\end{array}$ & $\begin{array}{l}0.0001 \\
0.0002\end{array}$ & $\begin{array}{l}0.0029 \\
0.0020\end{array}$ & $\begin{array}{l}0.0007 \\
0.0004\end{array}$ \\
\hline $\bar{y}_{3}$ & 0.0045 & -0.0060 & -0.0016 & $\sigma_{y_{3}}$ & 0.0001 & 0.0019 & 0.0004 \\
\hline
\end{tabular}




G Model
RIMNI-184; No. of Pages 7
M. Cacho-Perez et at. / Rev. int. métodos numér. cálc. diseño ing. 2016:xxx(xx):xxx-xxx

\section{Conclusiones}

Analizados los resultados experimentales obtenidos, la primera conclusión que merece la pena reseñar (por la relevancia que tiene sobre el resto de las interpretaciones de los datos resultantes\} es que sôlo se pueden obtener con precisión los parámetros modales que corresponden a los modos de vibración que seamos capaces de que corresponden a los modos de vibración que seamos capaces de
excitar.Aunque lógica, esta concl usiôn ha de estar siempre presente excitar. Aunque lógica, esta concl usión ha de estar siempre presente
tan to en el análisis como en las aplicaciones de los resul tados. También se debe tener en cuenta que la incertidumbre en los resul tados será mayor a medida que la participaciôn en la respuesta dinámica de los modos sea menor.

En segundo lugar, a partir de los resultados experimentales de la pasarela peatonal, se concluye que en general mediante los métodos de identificaciôn de sistemas dinámicos se va a conseguir precisiôn suficiente en la estimaciôn de las frecuencias propias y en los modos de vibraciôn normalizados respecto de la matriz de masa, y no así en otras magnitudes como los factores de amortiguamiento, masas generalizadas y modos de vibraciôn asociados a dichas masas.

También resaltar que estimar los modos de vibraciôn normalizados respecto de la matriz de masa tiene su dificul tad a partir de los registros experimentales, ya que en los métodos de identificación de sistemas no se tiene en ningún momento una expresiôn explícita de dicha matriz de masa.

Y por último, apuntarque la estimaciôn de los parámetros modales permite la simulaciôn de la respuesta dinámica de la estructur sin la necesidad de actualizar un modelo de elementos finitos de la estructura objeto de estudio (método que se conoce en la litela estructura objeto de estudio (método que se conoce en la lite-
ratura técnica como FEM Updating). Así mismo, también posibilita ratura técnica como FEM Updating). Así mismo, también posibilita
predecir su comportamiento dinámico frente a distintos tipos de solicitaciones a partir del modelo modal de la pasarela peatonal, por ejemplo para condiciones usuales o excepcionales de tráfico peatonal.

\section{Agradecimientos}

Este trabajo ha sido realizado en parte con la financiaciôn aportada al proyecto de investigaciôn BlA2011-28493-C02 por el programa de apoyo a Proyectos de Investigaciôn Fundamental No Orientad a del Ministerio de Ciencia e Innovaciôn. Los autores agradecen al Ayuntamiento de Valladolid y al Museo de la Ciencia su apoyo en las pruebas de campo.

\section{Bibliografia}

[1] J. de Sebastian, I.M. Diaz, C.M. Casado, A.V. Poncela, A. Lorenzana, Evaluation of acceleration estimates due to pedestrian loading on an in-service footbridge,

[2] A. Sestieri, S.R. Ibrahim, Analysis of emors and approximations in the use of [3] DJ. Ewirs, Modal Testing theory practice and application, Research Studies Press Ltd, 2000

[4] J.E. Mottershead, M.I. Friswell, Model updating in structural dynamics: A survey, Joumal of Sound and Vibration $165\{2\}\langle 1993\} 347-375$.

5] C.E. Ventura,j.F. Lord, M. Turek, R. Brincker, P. Andersen, E. Dascotte, Fem upda-
Intemational Conference on Structural Dynamics 〈EURODYN\}, Paris, France,

[6] D. Bernal, B. Gunes, Darnage localization in output-only systems: a flexibility based approach, en: Proceedings of the Intern

7] R. Brincker P. And Angeles, California, 2002 . modal analysis, en: Proceedings of the International Modal Analysis Conference (IMAC) XXI, Orlando, USA, 2003

[8] P. Femández, M. López Aenlle, L. Villa, R. Brincker, Scaling factor estimation using an optimized mass change strategy. part2: experimental results, en: ProCopenhagen, Denmark, 2007.

[9] M.M. Khatibi, M.R. Ashory. A. Malekjafarian, Scaling of mode shapes using mass-stiffness change method, en: Proceedings of the International Operational Modal Analysis Conference $\langle$ IOMAC\}, Copenhagen, Denmark, 2009.

[10] M. López-Aenlle, R. Brincker, F. Pelayo, A.F. Canteli, On exact and approximated formulations for scaling-mode shapes in operational modal analysi by mass and stiffness change, Journal of Sound and Vibration $331\{2012\}$
$622-637$.

[11] M. López-Aenlle, M.P. Fernández, R. Brincker, A. Fernández, Canteli, Scaling fac and Signal Processing 24\{2010\}3061-3074.

[12] E. Parloo, P. Verboven, P. Guill aume, M. Van Overmeire, Sensitivity-based mas normalization of mode shape estimates from output-only data, en: Proceeding of the Intemational Conference on structural System Identification, 2001.

(3) E. Paro, $16\{2002\} 757-767$

[14] J. Porras, J. de Sebastián, C. Casado, A. Lorenzana, Modal mass estimation from output-only data using oscillator assembly, Mechanical Systems and Signal

Processing 26 \{2011) $15-23$.
[15] S.R. Ibrahim, Randorn decrement technique for modal identification of structures, Journal of Spacecraft and Rockets $14\langle 11\}\langle 1977) 696-700$.

Pa. 183-198.

[17] J.N. Juang, J.E. Cooper, J.R. Wright, An eigensystern realization algorithm using data correl ations $\{e r a\} d c\}$ for modal parameter identification, Control Theory

[18] J.N. Juang, R.S. Pappa, An eigensystem realization algorithrn for modal para-

[19] J.N. Juang, M. Phan, L.G. Horta, R.W. Longman, Identification of observer/kalma filter markov parameters: Theory and experiments, Journal of Guidance, Con-

trol, and Dynamics $16\{2\}\{1993\} 320-329$.
[20] H. Lus, R. Betti, R.W. Longman, Identification of linear structural systems using earthquake - induced vibration data, Earthquake Engineering and Structural

21] H. Vold, J. Kundrat, G.T. Rocklin, R. Russell, A multiple-input modal estimation algorithm for mini computers, SAE Transactions 91 (1\}<1982\} 815-821.
K.F. Alvin, K.C. Park, Second-onder structural identif

space-based system identification, AIAA Joumal $32\{2\}\{1994\} 397-406$.

[23] M. de Angelis, H. Lus, R. Betti, R.W. Longman, Extracting physical parameter of mechanical models from identified state-space representations, Joumal of Applied Mechanics-Transactions of the ASME $69\{5\rangle\langle 2002\} 617-625$.

[24] D.H. Tseng, R.W. Longman, J.N. Juang, Identification of gyros copic and nongyroscopic second order mechnical systems includin
Advances in Astronautical Sciences $87\{1994\} 145-165$.

[25] D.H. Tseng, R.W. Longman, J.N. Juang, Identification of the structure of the damping matrix in second order mechanical systerns, Advances in Astronautica

Sciences 87 81994 ) 166-190.
[26] C.D. Yang, F.B. Yeh, Identification, reduction, and refinement of model parameters by the eigen systern realization al

[27] J.M.W. Brownjohn, A. Pavic, Experimental methods for estimating modal mass in footbridges using human-induced dynamic excitation, Engineering Structures $29\{2007\} 2833-2843$.

28] R.J. Allemang, Vibrations: Analytical and experimental modal analysis, en Short Course Notes UC-SDRL-CN-20-263-662, University of Cincinnati, 2000. [29] W.T. Thoms on, Theory of vibration with applications, Chapman \& Hall, London,
1993. 
"Estimación de las masas modales de una estructura en servicio mediante transformación en el espacio de estados"

M. Cacho Pérez, N. Frechilla y A. Lorenzana

Revista Internacional de Métodos Numéricos para Cálculo y Diseño en Ingenieria. http://dx.doi.org/10.1016/j.rimni.2016.02.002. Online publication date: 1-Jun-2016. 


\begin{tabular}{|c|c|c|}
\hline \multirow[t]{2}{*}{$\begin{array}{l}\text { G Model } \\
\text { RIMNI-185; No. of Pages } 6\end{array}$} & ARICLE IN PRESS & \\
\hline & Rev. int. métodos numér. cálc. diseño ing. 2016; $x x x(x x): x x x-x x x$ & \\
\hline s.t. & $\begin{array}{c}\text { Revista Internacional de Métodos Numéricos para } \\
\text { Cálculo y Diseño en Ingeniería }\end{array}$ & 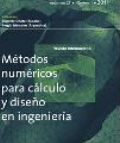 \\
\hline$\overline{\text { ELSEVIER }}$ & www.elsevier.es/rimni & \\
\hline
\end{tabular}

Estimación de las masas modales de una estructura en servicio mediante transformación en el espacio de estados

M. Cacho-Pérez*, N. Frechilla y A. Lorenzana

Escuela de Ingenierías Industriales, Universidad de Valladolid, Paseo del Cauce 59, 47011, Valladolid, Spain

\begin{tabular}{l} 
INFORM ACIÓN DEL ARTÍCULO \\
\hline Historia del artícuto: \\
Recibido el 15 de julio de 2015 \\
Aceptadoel 19 de febrero de 2016 \\
On-line el $x x x$ \\
\hline Palabras clave: \\
Pasarela peatonal \\
Sisterna dinámico \\
Modos escalados
\end{tabular}

Keywords:

Footbridge

Dynamic system

\begin{abstract}
R E S U M E N
Este trabajo se centra en presentar una metodología práctica para estimar los parámetros modales de estructuras en uso y se aplica a la pasarela peatonal del Museo de Ciencia de la ciudad de Valladolid, España. El trabajo consiste no sólo en calcular frecuencias propias y factores de amortiguamiento asociados a cada uno de los modos estimados, como proporcionan muchos programas comerciales a partir de los registros de aceleraciones de ensayos OMA (Operational Modal Analysis) y/o EMA (Experimental Modal Analysis), sino también calcular las masas generalizadas correspondientes a cada uno de los modo estimados de la estructura. Para ello, en primer lugar se obtiene una representación del sistema dinámico en el espacio de estados mediante la técnica SSI (Stochastic Subspace Identification) y en segundo lugar, mediante la adecuada matriz de transformación se llega a la representación que permite identificar los parámetros físicos del sistema (matrices de masa, amortiguamiento y rigidez), lo que permite obtener masas modales y/o modos normalizados respecto de la matriz de masa, principal novedad de la metodología propuesta.

(c) 2016 CIMNE (Universitat Politècnica de Catalunya). Publicado por Elsevier España, S.LU. Este es un artículo Open Access bajo la CC BY-NC-ND licencia (http://creativecommons.org/licencias/by-nc-nd

Estimation of modal masses for an in-service structure by transformation in the state space

A B S T R A C T

This paper focuses on estimating the modal parameters of in-service infrastructures, applied to the pedestrian footbridge of the Science Museum of the city of Valladolid (Spain). The work consists not only on determining natural frequencies and damping factors associated to each of the estimated modes, as many commercial software provide from acceleration measurements through OMA (Operational Modal Analysis) and/or EMA (Experimental modal Analysis), but al so to calculate the generalized masses corresponding to each of the estimated modes of the structure. For this purpose, firstly a dynamic representation of th system is obtained in the state space by SSI technique (Stochastic Subspace Identification) and secondly. by the appropriate transformation matrix, it leads to the representation that identifies the physical parameters of the system (matrices of mass, damping and stiffness), which allows to obtain modal masse and/or normalized mode shapes with respect to the mass matrix, which is the main contribution of the practical methodology presented in this paper.

(c) 2016 CIMNE (Universitat Politècnica de Catalunya). Published by Elsevier España, S.L.U. This is an open access article under the CC BY-NC-ND license (http://creativecommons.org/licenses/by-nc-nd/

\footnotetext{
* Autor para corres pondencia.

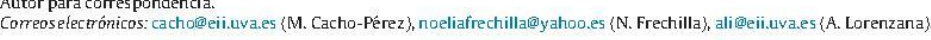

http://dx.doi.org/10.1016/j.rimni.2016.02.002

0213-1315/O 2016 CIMNE (Universitat Politècrica de Catalunya). Publicado por Elsevier España, S.L.U. Este es un artículo Open Access bajo la CC BY-NC-ND licencia 〈http:/ creativecommons.org/licencias; /by-nc-nd/4.0/\}

Cômo citar este artículo: M. Cacho-Pérez, et al. Estimación de las masas modales de una estructura en servicio mediante transformaciôn en el espacio de estados, Rev. int. métodos numér. cálc. diseño ing. 2016. http://dx.doi.org/10.1016/j.rimni.2016.02.002 


G Model
RIMNI-185; No. of Pages 6
2
M. Cacho-Pérez et al. /Rev. int métodos numér. cál. diseño ing. 2016:xxx(xx):xxx-xxx

\section{Introducción}

En la actualidad, el diseño y construcción de un gran número de obras de ingeniería industrial y civil, tales como chimeneas industriales, torres, edificios altos, puentes, pasarelas o viaductos, ya no está apenas limitada por los materiales -cada vez más resistentesy los sistemas constructivos -cada vez con mayor componente y los sistemas constructivos -cada vez con mayor componente
tecnológico-, lo que ha provocado que sean cada vez más esbeltas, ambiciosas y complejas.

Este hecho, junto con las actuales demandas sociales relativas a la percepciôn y confort, en algunos casos recogidas en las normativas y guías, suscitan la necesidad de integrar el cálculo dinámico de manera más sistemática en el análisis y diseño asistido por ordenador de estructuras esbeltas que son las más susceptibles de sufrir movimientos o vibraciones significativas.

Un caso particularmente relevante es el de las pasarelas peatonales, ya que generalmente son propensas a ser excitadas por las personas que las ocupan, apareciendo movimientos apreciables que incluso pueden llegar a ser molestos 0 , en algunos casos inadecua doso peligrosos desde el punto de vista de la resistencia estructural

Para facilitar la integraciôn del análisis dinámico es imprescindible disponer del mayor número posible de metodologías de cálculo que permitan la identificación de los parámetros más relevantes que definen el comportamiento de la estructura $[11,12]$.

Respecto a la metodología de cálculo dinámico, la técnica de análisis modal de estructuras es una de las más importantes, estando estructurada en tres fases principales: recolecciôn de datos, identificaciôn del sistema y por último estimaciôn de los parámetros modales $[8]$.

Es la segunda etapa, la de identificación del sistema, la que juega un papel crucial en el número y tipo de parámetros modales que se puedan estimar [10], basándose los métodos de identificaciôn clásicos en aplicar una excitaciôn al sistema y a continuaciôn registrar y procesar la correspondiente respuesta (Experimental Modal trar y procesar la

Las propiedades modales de una estructura obtenidas por simulaciôn (problema de valores propios) pueden ser diferentes de los valores experimentales debido principalmente a la suposiciôn asociada con el modelado analítico. Las propiedades dinámicas pueden medirse por los experimentos después de que haya concluido la construcción de la estructura. La masa de una estructura se puede estimar con bastante precisión mediante un simple cálculo, sin embargo la masa modal es una propiedad dinámica relacionada no embargo la masa modal es una propiedad dinámica relacionada no sôlo con la distribuciôn de la masa, sino también con las formas de la vibración. En este estudio la técnica de identificacion del

El método de identificación más utilizado en la actualidad se denomina SSI (Stochastic Subspace Identification). Este método se ha aplicado en muchas situaciones para extraer los parámetros modales de las estructuras existentes (por ejemplo puentes y edificios) les de las estructuras existentes (por ejemplo puentes y edificios)
solicitadas por la vibraciôn ambiental. Sin embargo, la informasolicitadas por la vibraciôn ambiental. Sin embargo, la informa-
ciôn modal que se obtiene de estos métodos de identificaciôn, es incompleta ya que proporciona estimaciones de frecuencias, factores de amortiguamiento y modos de vibración asociados, pero dichos modos no están normalizados respecto de la matriz de masa , dicho de otra forma, esos n ralizadas correspondientes a cada uno de los modos estimados [1]. Es por lo tanto muy interesante disponer de técnicas adicionales que a partir de toda la información obtenida al aplicar el método SSI nos permitan estimar todos los parámetros modales, incluidos las masas modales de cada modo de vibraciôn [2].

En las formulaciones deelementos finitos, la identificación de los parámetros físicos generalo para mas fisicos gentaces diferenciales de segundo orden del problema dinámico. Aunque el método más empleado consiste en el ajuste de los parámetros modales del sistema para calibrar un modelo de elementos finitos (FEM Updating) [7], un posible enfoque es identificar estos parámetros directamente a partir de los datos experimentales. e primer orden también ha recibido considerable atención pero como es bien sabido, si se parte de un espacio de estados modelo y se trata de identificar los parámetros del modelo de segundo orden, cuestiones como la no unicidad de la soluciôn complican la resoluciôn del problema inverso. Sin embargo, los parámetros físicos del modelo de segundo orden se pueden obtener mediante la resolución de un problema simétrico de valores propios $[3,6,9]$. El requisito mínimo es que en todos los grados de libertad se debe ubicar o bien un sensor 0 un actuador, con al menos una localizaubicar o bien un sensor o un actuador, con al menos una localiza-
ción de ubicaciôn conjunta con par sensor-actuador. De esta forma se consigue una solución para el problema inverso.

Indicar que el trabajo se ha organizado de la manera siguiente: en primer lugar, tras esta introducciôn, se presenta la metodología empleada A continuación se diserta sobre los resultados obtenidos y en el último apartado se presentan las principales conclusiones.

\section{Metodología}

Los parámetros físicos del problema modal y dinámico (modelos de segundo orden se pueden estimar mediante la resolución de un problema simétrico de valores propios complejos. El requisito mínimo para esta metodología es que todos los grados de libertad mínimo para esta metodología es que todos los grados de libertad
contengan o bien un sensor o bien un actuador con al menos un contengan o bien un sensor o bien un actuador con al menos un par sensor-actuador conjunto. De esta manera, aunque son posibles
otras configuraciones, el enfoque que se propone en este trabajo proporciona una solución sencilla y práctica al problema inverso.

\subsection{Formulación en el espacio de estados}

Una de las formulaciones bien conocidas para los sistemas dinámicos es la expresión matricial de la segunda ley de Newton, que una vez discretizada en el dominio espacial resulta:

$\mathbf{M} \ddot{\mathbf{u}}(t)+\mathbf{C} \dot{\mathbf{u}}(t)+\mathbf{K u}(t)=\mathbf{f}(t) ; \quad \mathbf{y}(t)=\ddot{\mathbf{u}}(t)$

donde $\mathbf{u}(t)$ indica el vector de desplazamientos nodales, con () y (?) representando la primera y segunda derivada respecto al tiempo respectivamente, el vector $\mathbf{f}(t)$ contiene las excitaciones externas que solicitan el sistema, las magnitudes $\mathbf{M}, \mathbf{C}$ y $\mathbf{K}$ son las matrices simétricas definidas positivas de masa, amortiguamiento y rigidez, respectivamente, para un sistema de $N$ grados de libertad, $y$ $\mathbf{y}(t)$ el vector respuesta del sistema, en nuestro caso de interés, las aceleraciones.

Otra formulaciôn consiste en la definiciôn del vector de estado $x(t)$ de manera que las ecuaciones del movimiento pueden escribirse mediante un sistema de ecuaciones diferenciales de primer orden de una forma más conveniente como:

$$
\left[\begin{array}{ll}
\mathbf{C} & \mathbf{M} \\
\mathbf{M} & \mathbf{0}
\end{array}\right] \dot{\mathbf{x}}(t)+\left[\begin{array}{ll}
\mathbf{K} & \mathbf{0} \\
\mathbf{0} & -\mathrm{M}
\end{array}\right] \mathbf{x}(t)=\left[\begin{array}{l}
\mathbf{I} \\
\mathbf{0}
\end{array}\right] \mathbf{f}(t) ; \quad \mathbf{x}(t)=\left[\begin{array}{l}
\mathbf{u}(t) \\
\dot{\mathbf{u}}(t)
\end{array}\right]
$$

La ventaja de escribir las ecuaciones del movimiento de esta forma es que el problema de valores propios asociado es simétrico y puede plantearse en forma matricial como:

$\left[\begin{array}{ll}\mathbf{C} & \mathbf{M} \\ \mathbf{M} & \mathbf{0}\end{array}\right]\left[\begin{array}{l}\psi \\ \psi \mathbf{\Lambda}\end{array}\right] \Lambda=\left[\begin{array}{ll}-\mathrm{K} & \mathbf{0} \\ \mathbf{0} & \mathbf{M}\end{array}\right]\left[\begin{array}{l}\psi \\ \psi \mathbf{\Lambda}\end{array}\right]$

donde $\psi=\left[\psi_{1} \psi_{2} \ldots \psi_{2 N}\right]$ es la matriz que contiene los autovectores del problema complejo de valores propios siguiente:

$\left(\lambda_{i}^{2} \mathbf{M}+\lambda_{i} \mathbf{C}+\mathbf{K}\right) \psi_{i}=0$

Cômo citar este artículo: M. Cacho-Pérez, et al. Estimación de las masas modales de una estructura en servicio mediante transformaciôn en el espacio de estados, Rev. int. métodos numér. cálc. diseño ing. 2016. http://dx.doi.org/10.1016/j.rimni.2016.02.002 
y $\Lambda$ es la matriz diagonal de valores propios complejos $\lambda_{i}=\zeta_{i} \pm j \omega_{i}$ (siendo $j=\sqrt{-1}, \omega_{i}$ la frecuencia en $r d / s$ y $\zeta_{i}$ se puede relacionar fácilmente con el factor de amortiguamiento).

Una vez que el problema de valores propios ha sido resuelto podemos reescribir la ecuación (2) mediante la siguiente transformaciôn. Resultando finalmente:

$$
\begin{aligned}
& \mathbf{x}(t)=\left[\begin{array}{l}
\psi \\
\psi \Lambda
\end{array}\right] \mathbf{z}(t) \\
& \dot{\mathbf{z}}(t)=\Lambda \mathbf{z}(t)+\psi^{\top} \mathbf{f}(t) \\
& \mathbf{y}(t)=\psi \Lambda^{2} \mathbf{z}(t)+\psi \Lambda \psi^{\top} \mathbf{f}(t)
\end{aligned}
$$

Denominaremos el sistema anterior (5) como modelo modal simétrico.

\subsection{Identificación del sistema dinámico}

La técnica de identificaciôn consta de dos fases bien definidas: en primer lugar la determinación del modelo de primer orden de sistema y en segundo lugar la transformación del modelo identificado al correspondiente modelo de segundo orden. A partir de los datos de aceleraciôn registrados en los sensores y de la fuerza aplicada mediante el actuador, es posible identificar (mediante el método SSI) una representaciôn en el espacio de estados en una base arbitraria, que se puede expresar como:

$$
\begin{aligned}
& \dot{\mathbf{x}}(t)=\mathbf{A}_{\mathbf{C}} \mathbf{x}(t)+\mathbf{B}_{\mathbf{C}} \mathbf{f}(t) \\
& \mathbf{y}(t)=\mathbf{C}_{C} \mathbf{x}(t)+\mathbf{D}_{\mathbf{C}} \mathbf{f}(t)
\end{aligned}
$$

donde $\mathbf{A}_{\mathrm{C}}, \mathbf{B}_{\mathrm{C}}, \mathbf{C}_{\mathrm{C}}$ y $\mathbf{D}_{\mathcal{C}}$ son las matrices del sistema en tiempo continuo. Ahora, si se considera la transformación $\mathbf{X}=\varphi \boldsymbol{\theta}$ el sistema en tiempo continuo puede escribirse en forma modal como se indica:

$$
\dot{\theta}(t)=\Lambda \theta(t)+\varphi^{-1} \mathbf{B}_{\mathbf{c}} \mathbf{f}(t)
$$$$
\mathbf{y}(t)=\mathbf{C}_{\mathbf{C}} \varphi \theta(t)+\mathbf{D}_{\mathbf{C}} \mathbf{f}(t)
$$

donde $\Lambda$ es la matriz que contiene los valores propios del modelo de espacio de estados identificado y $\varphi$ es la correspondiente matriz de vectores propios. Denominaremos a este sistema (7) como modelo modal no simétrico.

Si el sistema de primer orden (6) se identificó utilizando datos que realmente corresponden a un sistema que responde a la ecuaciôn de segundo orden (1), los modelos representados por las ecuaciones (5) y (7) son dos representaciones diferentes del mismo sistema dinámico. Por lo tanto, podemos buscar una matriz de transformaciôn $(\mathbf{T})$ que relacione ambas expresiones, es decir:

$$
\begin{aligned}
& \mathbf{T}^{-1} \Lambda \mathbf{T}=\Lambda \\
& \mathbf{T}^{-1} \varphi^{-1} \mathbf{B}_{\mathbf{C}}=\psi^{T} \\
& \mathbf{C}_{\mathbf{C}} \varphi \Lambda^{-2} \mathbf{T}=\psi
\end{aligned}
$$

Supongamos que el grado de libertad i-ésimo contiene un sensor y un actuador. El requisito de co-localizaciôn junto con el hecho de que la matriz $\mathbf{T}$ es diagonal permite determinar dicha matriz de manera unívoca mediante la siguiente expresiôn:

$\mathbf{C}_{\mathbf{C}}(i,:) \varphi \Lambda^{-2} \mathbf{T}^{2}=\left(\varphi^{-1} \mathbf{B}_{\mathbf{C}}\right)^{\top}$

Dependiendo de la configuración de sensores y actuadores la información relativa a un cierto grado de libertad está embebida ya sea en la matriz de entrada o en el matriz de salida. En este caso, la matriz de salida contiene información de los $N$ grados de libertad, con lo cual, los autovectores $\psi$ se calculan con la última de las expresiones indicadas en (8). Y una vez calculados dichos autovectores, las matrices de masa, amortiguamiento y rigidez se pueden obtener haciendo uso de las condiciones de ortogonalidad, éstas son:

$$
\begin{aligned}
& {\left[\begin{array}{l}
\psi \\
\psi \Lambda
\end{array}\right]^{\top}\left[\begin{array}{ll}
\mathbf{C} & \mathbf{M} \\
\mathbf{M} & \mathbf{0}
\end{array}\right]\left[\begin{array}{l}
\psi \\
\psi \Lambda
\end{array}\right]=\mathbf{I}} \\
& {\left[\begin{array}{l}
\psi \\
\psi \Lambda
\end{array}\right]^{\top}\left[\begin{array}{ll}
\mathbf{K} & \mathbf{0} \\
\mathbf{0} & -\mathbf{M}
\end{array}\right]\left[\begin{array}{l}
\psi \\
\psi \Lambda
\end{array}\right]=-\Lambda}
\end{aligned}
$$

Lo que implica que tras las correspondientes operaciones algebraicas podemos estimar los parámetros físicos del sistema dinámico según:

$$
\begin{aligned}
& \mathbf{M}=\left(\psi \Lambda \psi^{T}\right)^{-1} \\
& \mathbf{C}=-\mathbf{M} \psi \Lambda^{2} \psi^{T} \mathbf{M} \\
& \mathbf{K}=-\left(\psi \Lambda^{-1} \psi^{T}\right)^{-1} ; \psi \psi^{T}=\mathbf{0}
\end{aligned}
$$

Y por último, como es sabido, conocida la matriz de masa, la determinación de las masas generalizadas/modales o de los modos de vibraciôn normalizados es algo inmediato.

\section{Resultados numéricos}

Una vez expuesta la metodología, procedemos a la validaciôn de la misma presentando un primer problema numérico pero suficientemente general (validaciôn), para después aplicar dich metodología al análisis de un caso general más complejo (ejemplo) y de una estructura real actualmente en uso.

\subsection{Validación}

El problema que vamos a plantear es el de una viga biapoyada de longitud $\mathrm{L}=10.4 \mathrm{~m}$, módulo de Young $\mathrm{E}=2.1 \cdot 10^{11} \mathrm{~Pa}$, densidad $\rho=7850.0 \mathrm{~kg} / \mathrm{m}^{3}$, inercia $\mathrm{lz}=604 \cdot 10^{-8} \mathrm{~m}^{4}$ y secciôn transversal de área $\mathrm{A}=53.8 \cdot 10^{-4} \mathrm{~m}^{2}$. También se supone un factor de amortiguamiento de $5 \%$ para cada uno de los modos de vibración de dicha viga. Se simula mediante herramienta matemática de manera que se obtienen las aceleraciones en las posiciones $(1 / 4,1 / 2,3 / 4)$. (sensores 1,2 y 3 , respectivamente) cuando se aplica una fuerza externa conocida $\left.(f t)=10^{2} \cdot \sin (2 \pi t)\right)$ en la misma sección donde se ubica el sensor 1 (para el grado de libertad 1, corresponde el par sensor-actuador?

A continuación, se sigue la metodología de este trabajo. Primero se identifica el sistema en base a un modelo de espacio de estados a partir de los datos de respuesta. En segundo lugar se determinan los factores de escala dados por la matriz diagonal $\mathbf{T}$ y los vectores pro-

\begin{tabular}{|c|c|c|c|c|c|c|}
\hline \multirow[b]{2}{*}{ modo } & \multicolumn{3}{|c|}{ Estimado } & \multicolumn{3}{|c|}{ Analítico } \\
\hline & $\mathrm{f}_{\mathrm{i}}\langle\mathrm{Hz}\rangle$ & $\zeta(\%)$ & $M_{\{}\langle\mathbf{k g}\}$ & $\mathrm{f}_{\mathrm{i}}\langle\mathrm{Hz}\rangle$ & $\zeta_{\langle(\%)}$ & $\mathrm{M}_{1}\{\mathbf{k g}\}$ \\
\hline 1 & 2. & 0.5 & 219.7 & 2 & 0.5 & 219.6 \\
\hline 2 & 10.06 & 0.5 & 216.9 & 10.07 & 0.5 & 219.6 \\
\hline 3 & 22.61 & 0.5 & 194.1 & 22.65 & 0.5 & 219.6 \\
\hline
\end{tabular}
pios $\psi$. Después se estiman las matrices de masa, amortiguamiento y rigidez del modelo de segundo orden (M, C y K, respectivamente). Por último se calculan todos los parámetros modales.

En la tabla 1 se recogen los resultados estimados de los parámetros modales: frecuencias propias $\left(f_{i}\right)$, factores de amortiguamiento $\left(\zeta_{i}\right)$ y masas generalizadas $\left(M_{i}\right)$ asociados a los tres primeros modos de vibraciôn de la viga, asimismo, se indica la soluciôn analítica correspondiente.

La tabla 2 muestra los modos normalizados respecto de la matriz de masa correspondientes a los tres primeros modos de vibración. Se observa, al igual que en la tabla 1, que los resultados para el

Tabla 1

Viga biapoyada. Paŕmetros modales 
G Model

RIMNI-185; No. of Pages 6

ARTICLEE IN PRESS

M. Cacho-Pérez et al. / Rev. int métodos numér. cálc. diseñono ing. 2016:xxx(xx):xxx-xxx

Tabla 2
Viga biapoyada. Modos de vibración normalizados

\begin{tabular}{lllllllr}
\hline & \multicolumn{3}{c}{ Estimado } & & \multicolumn{3}{c}{ Analítico } \\
\cline { 2 - 4 } \cline { 5 - 7 } modo & 1 & \multicolumn{1}{c}{2} & \multicolumn{1}{c}{3} & & \multicolumn{2}{l}{2} & \multicolumn{1}{l}{3} \\
\hline$y_{1}$ & 0.0477 & -0.0674 & 0.0506 & & 0.0477 & -0.0674 & 0.0477 \\
$y_{2}$ & 0.0675 & 0.0 & -0.0718 & & 0.0675 & 0.0 & -0.0675 \\
$y_{3}$ & 0.0477 & 0.0674 & 0.0506 & & 0.0477 & 0.0674 & 0.0477 \\
\hline
\end{tabular}

Tabla 3
Ejemplo. Viga continua. Parámetros modales

\begin{tabular}{|c|c|c|c|c|c|c|}
\hline \multirow[b]{2}{*}{ modo } & \multicolumn{3}{|c|}{ Estimado } & \multicolumn{3}{|c|}{ Analítico } \\
\hline & $f_{i}\langle\mathrm{~Hz}\rangle$ & $\zeta_{\{}(\%)$ & $\mathrm{M}_{\mathrm{i}}\langle\mathrm{kg}\}$ & $\mathrm{f}_{\mathrm{i}}\langle\mathrm{Hz}\rangle$ & $\zeta_{i}(\%)$ & $M_{i}\langle\mathrm{~kg}\rangle$ \\
\hline 1 & 3.75 & 0.50 & 146.6 & 3.75 & 0.50 & 146.5 \\
\hline 2 & 5.93 & 0.50 & 154.1 & 5.93 & 0.50 & 154.6 \\
\hline 3 & 11.69 & 0.49 & 116.3 & 11.69 & 0.50 & 124.9 \\
\hline 4 & 15.80 & 0.57 & 151.7 & 15.55 & 0.50 & 119.8 \\
\hline
\end{tabular}

tercer modo de vibración son poco precisos, esto se debe a que el tipo de carga aplicada en el ejemplo no es capaz de excitar el terce modo. Hubiera sido más conveniente haber excit con contenido variado en frecuencias.

\subsection{Ejemplo}

A continuación se va a mostrar la metodología de este trabajo para identificar el modelo modal de la viga continua de tres vanos, indicada en la figura 1 la longitud de cada uno de vanos, indicada en la figura 1 . La longitud de cada uno de los vanos es: $L_{1}=8 \mathrm{~m}, \mathrm{~L}_{2}=10 \mathrm{~m}$ y $\mathrm{L}_{3}=5 \mathrm{~m}$. Las propiedades del
material y de la sección son respectivamente: môdulo de Young $\mathrm{E}=2.1 \cdot 10^{11} \mathrm{~Pa}$, densidad $\rho=7850 \mathrm{~kg} / \mathrm{m}^{3}$, área de la secciôn transversal $A=53.8 \cdot 10^{-4} \mathrm{~m}^{2}$ y momento de inercia $\mathrm{l}_{\mathrm{z}}=604.0 \cdot 10^{-8} \mathrm{~m}^{4}$.

De la resolución analítica del problema de análisis modal de dicha viga se obtienen los modos de vibración y frecuencias propias que se muestran en la figura 2 . A continuación, se aplica una fuerza de tipo armónico $\left(f_{1}(t)=10^{2} \cdot \sin (2 \pi t)\right\}$ en la sección que indica la figura 1. Asimismo, se asume de nuevo un factor de amortiguamiento $\zeta_{i}=0.5 \%$ para cada uno de los modos de vibraciôn y se

calcula la respuesta dinámica de la estructura mediante el método de superposición modal (se consideran los seis primeros modos calculados anteriormente) con el objeto de generar los registros de aceleraciones en los puntos de la estructura indicados en la figura 1 de un minuto de duraciôn con un tiempo de muestreo $\mathrm{T}_{\mathrm{s}}=0.005 \mathrm{~s}$, para posteriormente emplear dichos registros, junto con el de la fuerza aplicada, como punto de partida en la aplicación de la metodología objeto de este trabajo.

Es decir, la idea es suponer que solamente contamos con la información recogida en los registros de aceleraciones y el registro de fuerza aplicada, según el esquema de la figura 1 . Se identifica el sistema y con el modelo obtenido en el espacio de estados se aplica la metodología expuesta y se obtienen las estimaciones que se resumen a continuación.

En la tabla 3 se recogen los resultados estimados de las frecuencias propias y los factores de amortiguamiento asociados a los cuatro primeros modos de vibración de la estructura y también los resultados analíticos correspondientes.
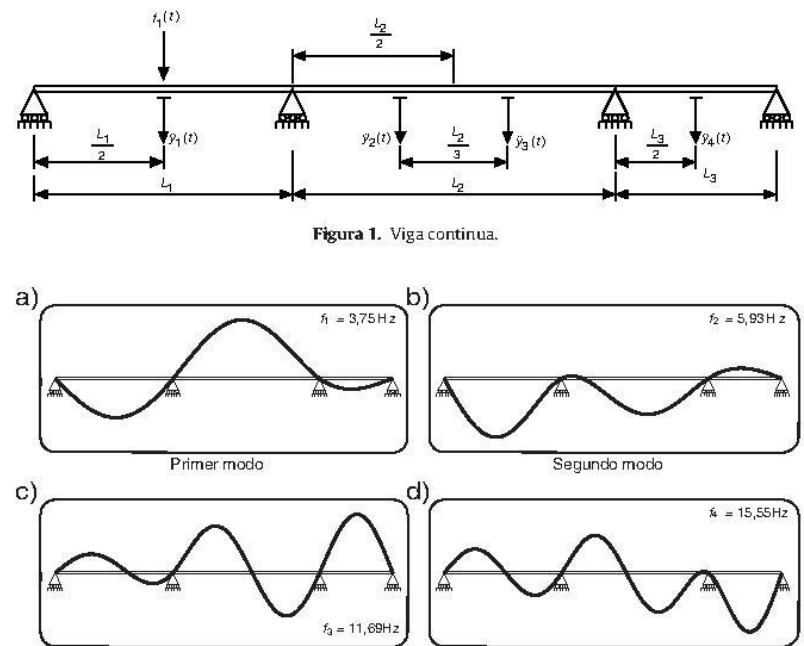

ercermodo

Cuarto modo

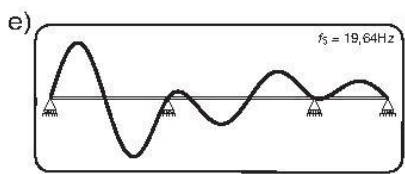

Quinto modo

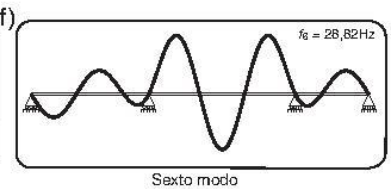

Figura 2. Viga continua. Modos de vibración.

Cómo citar este artículo: M. Cacho-Pérez, et al. Estimaciôn de las masas modales de una estructura en servicio mediante transformaciôn en el espacio de estados, Rev. int. métodos numér. cálc. diseño ing. 2016. http://dx.doi.org/10.1016/j.rimni.2016.02.002 


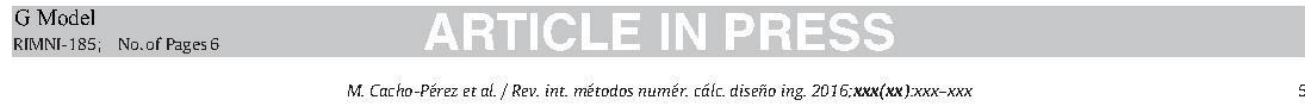

Tabla 4

\begin{tabular}{|c|c|c|c|c|c|c|c|c|}
\hline \multirow[b]{2}{*}{ modo } & \multicolumn{4}{|c|}{ Estimado } & \multicolumn{4}{|c|}{ Analítico } \\
\hline & 1 & 2 & 3 & 4 & 1 & 2 & 3 & 4 \\
\hline & -0.4783 & -0.8203 & 0.1317 & 0.0707 & -0.4783 & -0.8203 & 0.1315 & 0.0485 \\
\hline$y_{2}$ & 0.6467 & -0.2691 & 0.5576 & 0.3871 & 0.6467 & -0.2690 & 0.5578 & 0.3632 \\
\hline$y_{3}$ & 0.5797 & -0.4832 & -0.3897 & -0.5288 & 0.5797 & -0.4831 & -0.3902 & -0.5229 \\
\hline$y_{4}$ & -0.1297 & 0.1456 & 0.7210 & -0.7520 & -0.1297 & 0.1456 & 0.7206 & -0.7696 \\
\hline
\end{tabular}

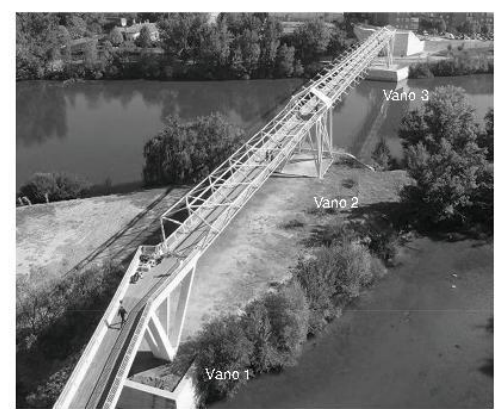

Figura 3. Vista general de la estructura en estudio.

Iabla 4 muestra los cuatro primeros modos de vibraciôn de la viga continua, estimados y teôricos. Se observa que los resultados para el tercer y cuarto modo de vibraciôn difieren ligeramente de los analíticos. Esto puede deberse a que su participación en la respuesta dinámica bajo la fuerza armónica propuesta es pequeña y por lo tanto la incertidumbre grande.

\subsection{Aplicación a un caso real}

En esta sección se describe la identificación de los parámetros modales de la pasarela peatonal del Museo de la Ciencia de la ciudad de Valladolid. Esta identificaciôn consistiô en un análisis moda experimental del vano de la estructura más sensible a las vibraciones inducidas por el tránsito de peatones.

\subsubsection{Descripción de la estructura}

la estructura estudiada es una pasarela peatonal sobre el río Pisuerga que une el Museo de la Ciencia con el centro de la ciudad. Construida en el año 2004, es una estructura de 234 metros compuesta de cuatro vanos: tres formados por barras metálicas tubulares y uno de hormigôn. El vano principal (vano 3 en la fig. 3) tiene 111 metros y cuenta con un sistema de tendones externos postensados. El segundo vano (vano 2 ) tiene 51 metros y los otros dos vanos son más cortos y rígidos. El sistema de tendones externo del vano 3 hace que sea suficientemente rígido. Sin embargo, el vano 2 (fig. 4), es más sensible a excitaciones dinámicas producidas por humanos. En dicho vano se al canzan niveles de vibraciôn que, según la opiniôn de los usuarios, a veces son molestos, particularmente cuando un corredor cruza el puente.

\subsubsection{Análisis modal experimental}

La identificaciôn de los parámetros modales de la estructura mediante OMA tiene el inconveniente de no identificar la masa modal, parámetro necesario para obtener el modelo modal comple to y para realizar las estimaciones de la respuesta utilizando las guías y normativas. Por ello es necesario utilizar otros métodos para

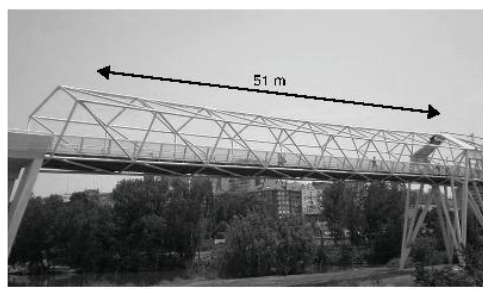

Figura 4. Vista del Vano 2

Tabla 5
Excitaciones inducidas en la pas arela

\begin{tabular}{lllll}
\hline prueba & excitación & frecuencias & Burt. & Nivel \\
\hline 1 & Chirp & $1-15 \mathrm{~Hz}$ & $0.95 \%$ & $0.4 \mathrm{~V}$ \\
2 & Chirp & $1-15 \mathrm{~Hz}$ & $0.95 \%$ & $0.4 \mathrm{~V}$ \\
3 & Chirp & $1-15 \mathrm{~Hz}$ & $0.95 \%$ & $0.4 \mathrm{~V}$ \\
4 & Chirp & $2.5-4.5 \mathrm{~Hz}$ & $0.95 \%$ & $1.0 \mathrm{~V}$ \\
5 & Chirp & $2.5-4.5 \mathrm{~Hz}$ & $0.95 \%$ & $1.0 \mathrm{~V}$ \\
6 & Chirp & $2.5-4.5 \mathrm{~Hz}$ & $0.95 \%$ & $0.1 \mathrm{~V}$ \\
7 & Chirp & $2.5-4.5 \mathrm{~Hz}$ & $0.95 \%$ & $0.5 \mathrm{~V}$ \\
8 & Chirp & $2.5-4.5 \mathrm{~Hz}$ & $0.95 \%$ & $0.3 \mathrm{~V}$ \\
9 & Chirp & $2.5-4.5 \mathrm{~Hz}$ & $0.95 \%$ & $0.7 \mathrm{~V}$ \\
\hline
\end{tabular}

Tabla 6

Valores medios y desviación típica de los parámetros modales estimados

\begin{tabular}{lllllrr}
\hline modo & $\overline{\mathrm{f}}_{\mathrm{i}}\langle\mathrm{Hz}\rangle$ & $\sigma_{\mathrm{f}_{\mathrm{i}}}\langle\mathrm{Hz}\rangle$ & $\bar{\zeta}_{\mathrm{i}}(\%)$ & $\sigma_{\zeta_{1}}\langle \%\rangle$ & \multicolumn{1}{c}{$\overline{\mathrm{M}}_{\mathrm{i}}\{\mathrm{kg}\rangle$} & $\sigma_{\mathrm{M}_{\mathrm{i}}\langle\mathrm{kg}\rangle}$ \\
\hline 1 & 3.50 & 0.0088 & 0.64 & 0.3630 & 17605.0 & 1028.0 \\
2 & 7.32 & 0.1882 & 4.45 & 3.5991 & 13120.3 & 3656.1 \\
3 & 9.23 & 0.1073 & 1.43 & 1.1121 & 149759.9 & 45246.8 \\
\hline
\end{tabular}

estimar este parámetro, siendo lo más inmediato hacer un EMA en el que la fuerza de excitación es conocida.

La fuerza de excitación empleada para este caso se generó con un excitador electrodinámico de APS Dynamics modelo 400 en modo inercial colocado en el centro del vano. La fuerza inducida por el excitador se calculó midiendo la aceleración de la masa inercial y multiplićndolo por su magnitud $(30.4 \mathrm{~kg}\}$ Se utilizó un barrido continuo en frecuencia entre 2.5 y $4.5 \mathrm{~Hz}$. Para los registros de la respuesta se colocaron tres acelerômetros piezoeléctricos en la línea central del vano. Este método tiene la ventaja de conocer la exci tacióny el inconveniente de que se necesita un excitador capaz de hacer vibrar la estructura con suficiente intensidad como para se percibida por los acelerómetros.

\subsubsection{Resultados experimentales}

Se llevaron a cabo un total de nueve pruebas ( toma de datos). En la tabla 5 se indican los ajustes del excitador en cada uno de ellos.

La tabla 6 recoge los valores medios estimados de las frecuencias propias $\left(f_{i}\right\}$, los factores de amortiguamiento $\left(\bar{\zeta}_{i}\right\}$ y las masas generalizadas $\left(M_{i}\right)$ correspondientes a los tres primeros mas a de cada una de dichas magnitudes: frecuencias $\left(\sigma_{f_{i}}\right\}$, factores de 


\begin{tabular}{|c|c|c|c|c|c|c|c|}
\hline $\begin{array}{l}\text { G Mode } \\
\text { RIMNI-18 }\end{array}$ & 5; No. & Pages 6 & & & A & 31 & 늘 \\
\hline 6 & & & & M. Cach & o-Pérez et & al. /Rev. it & $t$ métc \\
\hline $\begin{array}{l}\text { Tabla } 7 \\
\text { Pasarela }\end{array}$ & dos de & vibración & & & & & \\
\hline modo & 1 & 2 & 3 & modo & 1 & 2 & 3 \\
\hline $\begin{array}{l}\bar{y}_{1} \\
y_{2} \\
y_{3}\end{array}$ & $\begin{array}{l}0.5146 \\
1.0000 \\
0.7636 \\
\end{array}$ & $\begin{array}{r}1.0000 \\
0.3114 \\
-0.7001 \\
\end{array}$ & $\begin{array}{r}-0.9643 \\
0.8966 \\
-0.5073 \\
\end{array}$ & $\begin{array}{l}\sigma_{y_{1}} \\
\sigma_{y_{2}} \\
\sigma_{y_{3}}\end{array}$ & $\begin{array}{l}0.0067 \\
0.0000 \\
0.0067 \\
\end{array}$ & $\begin{array}{l}0.0000 \\
0.0636 \\
0.1812 \\
\end{array}$ & $\begin{array}{l}0.0801 \\
0.1352 \\
0.1376 \\
\end{array}$ \\
\hline $\begin{array}{l}\text { bla } 8 \\
\text { is arel }\end{array}$ & des & Vividatur & 10. & & & & \\
\hline modo & 1 & 2 & 3 & modo & 1 & 2 & 3 \\
\hline $\begin{array}{l}\bar{y}_{1} \\
\bar{y}_{2} \\
\bar{y}_{3}\end{array}$ & $\begin{array}{l}0.0039 \\
0.0075 \\
0.0058\end{array}$ & $\begin{array}{r}0.0090 \\
0.0028 \\
-0.0062\end{array}$ & $\begin{array}{r}-0.0026 \\
0.0024 \\
-0.0013\end{array}$ & $\begin{array}{l}\sigma_{y_{1}} \\
\sigma_{y_{2}} \\
\sigma_{y_{3}}\end{array}$ & $\begin{array}{l}0.0001 \\
0.0002 \\
0.0002\end{array}$ & $\begin{array}{l}0.0013 \\
0.0006 \\
0.0014\end{array}$ & $\begin{array}{l}0.0004 \\
0.0004 \\
0.0004\end{array}$ \\
\hline
\end{tabular}

amortiguamiento $\left(\sigma_{\zeta_{i}}\right)$ y masas generalizadas $\left(\sigma_{M_{i}}\right)$. Se comprueba que el método de identificaciôn del sistema dinámico es preciso para estimar frecuencias de vibraciôn, pero respecto a los resultados de amortiguamiento y masas generalizadas hay una variación importante.

A continuaciôn, en la tabla 7 se indican los tres primeros modos de vibraciôn asociados a las masas generalizadas estimadas en la tabla anterior (tabla 6), mediante valor medio y desviaciôn de cada una de las coordenadas. Precisamente a la vista de la desviación se puede concluir una mayor incertidumbre en la estimación de los $\operatorname{modos} 2$ y 3 .

Por último, se indica en la tabla 8 los modos de vibraciôn normalizados respecto de la matriz de masa, en base a valores medios y desviaciôn típica de las coordenadas medidas. Estos modos de vibraciôn serían los que hay que utilizar para simular la respuesta dinámica de la pasarela mediante el método de superposiciôn modal empleando simplemente los tres primeros modos de vibraciôn de la estructura.

\section{Conclusiones}

Analizados los resultados experimentales obtenidos, la primera conclusiôn que merece la pena reseñar, por la relevancia que tiene sobre el resto de las interpretaciones de los datos resultantes, es que sólo se pueden obtener con precisión los parámetros modales que corresponden a los modos de vibración que seamos capaces de excitar. Aunque lógica, esta conclusiôn ha de estar siempre presente tanto en la planificación de las pruebas como en el postprocesado de los registros.

Relativo a la precisión en la estimaciôn de los modos de vibraciôn normalizados respecto a la matriz de masa, se considera que es suficiente, teniendo en cuenta además la dificultad que tiene su obtención a partir de los registros experimentales, ya que en los métodos de identificación de sistemas no se tiene en ningún momento una expresiôn explícita de la matriz de masa.

También se obtiene buena precisiôn en la determinaciôn de las frecuencias propias, siendo menor en otras magnitudes como los factores de amortiguamiento, las masas generalizadas o los modos de vibración. vibraciôn normalizados respecto a la matriz de masa, nos permiten la simulación de la respuesta de la pasarela peatonal y predecir su comportamiento dinámico frente a distintos tipos de solicitaciones (condiciones usuales o excepcionales de tráfico). Asimismo, tal y como comentamos en la introducción, nos permite el calibrado y como comentamos en la introducción, nos permite el calibrado
de dichos parámetros en las formulaciones de elementos finitos (FEM Updating) ya que son identificados directamente a partir de los datos experimentales.

La principal ventaja respecto a otros métodos de escalado de los modos [5,8,11] radica en que con una mínima informaciôn experimental respecto a la entrada (un solo canal coincidente con el correspondiente registro de salida\} y sin necesidad de modelos computacionales complejos, con tan solo las transformaciones matemáticas indicadas, se consigue determinar las masas modales de los modos excitables.

\section{Agradecimientos}

Este trabajo ha sido realizado en parte con la financiación aportada al proyecto de investigación BlA2011-28493-C02 por el programa de apoyo a Proyectos de Investigación Fundamental No Orientada del Ministerio de Ciencia e Innovación. Asimismo, los autores agradecen al Ayuntamiento de Valladolid y al Museo de la Ciencia su apoyo en las pruebas de campo.

\section{Bibliografía}

[1] K.F. Alvin, K.C. Park, Second-order structural identification procedure via state-

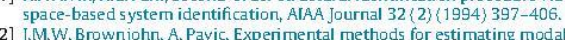
in footbridges using hurnan-induced dynamic excitar estimating modal mas res $29\{2007\} 2833-2843$.

[3] M. de Angelis, H. Lus, R. Betti, R.W. Longman, Extracting physical parameter of mechanical models from identified state-s pace representations, Journal of Applied Mechanics-Transactions of the ASME 69 (5) $\{2002\} 617-625$.

4] J. de Sebastian, I.M. Diaz, C.M. Casado, A.V. Poncela, A. Lorenzana, Evaluation of

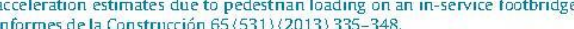
[5] D.J. Ewins, Modal Testing: theory, practice and application, Research Studies Press Ltd., 2000.

[6] H. Lus, M. De Angelis, R. Betti, R.W. Longman, Constructing second-order models of mechanical systems from identified state space realizations. part i Theoretical discussions, Journal of Engineering Mechanics-ASCE 129 (5) (2003) 7] J.E. Motterste

[7] Eey, Journsead, M.I. Friswell, Model updating in structural dynarnics: A survey, Journal of Sound and Vibration 165 \{2\} (1993) 347-375.
[8] J.A. Porras, J. de Sebastian, C.M. Casado, et al., Modal mass estimation from output-only data using oscillator assembly, Mechanical Systems and Signal

[9] A. Reggio, M. De Angelis, R. Betti, A state-space methodology to identify modal and physical parameters of non-viscously damped systems, Mechanical Sys-
terms and Signal Processing $41<1-2\}<2013) 380-395$

[10] A. Sestieri, S.R. Ibrahim, Analysis of errors and approximations in the use of A modal coordinates, Journal of Sound and Vibration $177\{2\}\{1994\} 145-157$.
ing

[11] L.M.V.llla García,Application of optimizationtechniques for determining moda parameters by means of frequency response functions, Rev. Int. Mét. Nurm. Cálc

[12] L.M. Villa García, Determination of modal parameters by means of the overall
adjustrment of the response, Rev. Int. Mét. Num. Cálc. Dise. Ing. $25\{3\}\langle 2009\}$ adjustment 
"Modal and dynamic analysis of a footbridge in service"

N. Frechilla, M. Cacho Pérez y A. Lorenzana

Revista Internacional de Métodos Numéricos para Cálculo y Diseño en Ingenieria. http://dx.doi.org/10.23967/j.rimni.2017.8.004. 


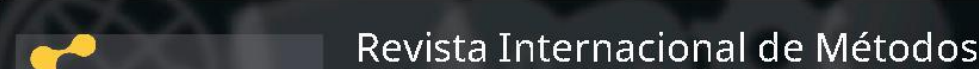 SCIPEDIA Numéricos para Cálculo y Diseño en Ingeniería

\section{Modal and dynamic analysis of a footbridge in service}

\author{
N. Frechilla ${ }^{1}$, M. Cacho-Pérez ${ }^{1}$, A. Lorenzana ${ }^{1}$ \\ 1 Escuela de Ingenierfas Industriales, Universidad de Valladolid, Paseo del Cauce 59, 47011, Valladolid, Spain
}

\section{Abstract}

Civil engineering constructions like pedestrian footbridges are structures increasingly slender, ambitious and complex in which the dynamic effects caused by the loads to which they are subjected (pedestrians) may induce service problems and / or significant or repetitive amplifications of the efforts and deformations. This fact together with the current social demands related to the perception and comfort raise the need to integrate the analysis and computer-aided design in the evaluation of the vibrations generated by the ommercial programs, their use has not proliferated as much as in other engineering fields due to the existence of simplified methodologies in numerous national and international quidelines and regulations and the difficulty of making realistic models in the case of complex structures. However, the knowledge generated by the analysis of this type of numerical models would be especially useful in the earlier phases of the design of similar structures, so much initial as of the necessary modifications to solve existing dynamic problems, since it is in these stages when the best solution could be applied from both a technical and an economic point of view. This article describes the process of generation and updating of the mechanical finite element model (FEM) of a footbridge in service and the simulation of two types of load: an excitation generated by a shaker and a pedestrian load, contrasting the results generated by the numerical model with those obtained experimentally.

\section{OPEN ACCESS}

Accepted: $27 / 06 / 2017$

Submitted: $28 / 04 / 2017$

10.23967/j.rimni.2017.8.004

Keywords: Pedestrian footbridge

vibrations

finite elements

\section{Resumen}

Obras de ingeniería civil como las pasarelas peatonales son estructuras cada vez más esbeltas, ambiciosas y complejas en las que los efectos dinámicos producidos por las solicitaciones a las que están sometidas (peatones) pueden inducir problemas de servicio y/o amplificaciones importantes o repetitivas de los esfuerzos y deformaciones. Ésto junto con las actuales demandas sociales relativas a la percepción y confort suscitan la necesidad de integrar el análisis y diseno asistido por ordenador en la evaluación de las vibraciones generadas por las cargas a las que están sometidas. El empleo de los programas comerciales de elementos finitos no ha proliferado tanto como en otros campos de la ingenierfa por la existencia de metodologlas simplificativas en numerosas guras y nomativas nacionales e internacionales y por la dificultad de hacer modelos realistas en el caso de estructuras complejas. Sin embargo, el conocimiento generado por el análisis de este tipo de modelos numéricos resultaría especialmente útil en las fases más tempranas del diseño de estructuras similares tanto inicial como de las modificaciones necesarias para solucionar problemas dinámicos existentes, ya que es en estas etapas cuando se podrfa aplicar la mejor solución desde un punto de vista técnico económico. En el presente artculo se describe el proceso de generación y ajuste del modelo mecánico de una pasarela actualconte econ servicio y simulación de la solicitación de dos tipos de carga: una excitación generada por un shaker y una carga peatonal,

Palabras clave: Pasarela peatonal, vibraciones, elementos finitos, ajuste modelo mecánico.

\section{Introducción}

El diseno y construcción de un gran número de obras de ingenierfa industrial y civil ya no está apenas limitada por los materiales -cada vez más resistentes-y los sistemas constructivos -cada vez con mayor componente tecnológico- lo que ha provocado que en la actualidad éstas sean más esbeltas, ambiciosas y complejas.

Este hecho, junto con las actuales demandas sociales relativas a la percepción y confort suscitan la necesidad de integrar el análisis y diseho asistido por ordenador en la evaluación de la respuesta dinámica de estructuras esbeltas que son las más susceptibles de sufrir movimientos o vibraciones significativas.

Un caso particularmente relevante es el de las pasarelas peatonales, ya que generalmente son propensas a ser excitadas por las personas que las ocupan, pudiendo los efectos dinámicos producidos inducir, además de problemas de servicio, amplificaciones importantes y repetitivas de los esfuerzos y deformaciones que pueden afectar a la seguridad resistente o la vida a fatiga de la estructura.

Aunque las normativas recogen diversos criterios de evaluación de estos fenómenos, también reflejan la dificultad de evaluar con precisión el comportamiento dinámico de las estructuras, exigiendo en algunos casos un estudio dinámico riguroso. 
La simulación mediante programas basados en el análisis de elementos finitos es una de las herramientas de cálculo con mayor profusión ya que los actuales softwares son muy competitivos respecto al ahorro de tiempo y costes. Sin embargo en el caso de análisis dinámico de estructuras no ha proliferado tanto debido a la existencia de normativás y gufas nacionales e internacionales [13] que proporcionan metodologras simplificativas, que siendo útiles para la acotación del problema dinámico no ofrecen la enome versatilidad del análisis numérico.

En general las predicciones logradas con el uso de modelos analiticos, especialmente en el caso de estructuras complejas como el que nos ocupa, difieren de los resultados obtenidos mediante ensayos experimentales por lo que es necesario efectuar un ajuste del que nos ocupa, difieren de los resultados obtenidos mediante ensayos experimentales por lo que es necesario efectuar un ajuste del modelo de elementos finitos (denominado en inglés FEM updating), mejorando las hipotesis efectuadas y aproximando sus propiedades a las de la estructura real. Aunque los datos experimentales poseen errores que puedan provocar una baja correlación entre éstos y las predicciones, la teorra de experimental son los errores en el modelo [4]

En el caso que nos ocupa se emplean los resultados experimentales procedentes de un análisis modal operacional, en inglés Operational Modal Analysis (OMA), realizado en la pasarela completa y un análisis modal clásico, del inglés Experimental Modal Analysis (EMA), realizado en el vano más sensible a excitaciones dinámicas producidas por humanos (vano 2), para conseguir que nuestro modelo numérico se ajuste lo mejor posible a la realidad. Los parámetros modales que identifican el comportamiento dinámico de la pasarela objeto de estudio (pasarela del Museo de la Ciencia de Valladolid) se han obtenido en estudios anteriores [5-7].

Una vez ajustado el modelo analítico (creado mediante el software de elementos finitos ANSYS) se han obtenido las respuestas dinámicas simuladas, en forma de aceleraciones, al aplicar la excitación generada durante el EMA y una carga peatonal según el modelo convencional de flujo de peatones [1]. Los resultados numéricos se han contrastado con los experimentales a fin de validar e comportamiento dinámico de la estructura modelizada.

El presente artículo se ha organizado del siguiente modo: en primer lugar, tras esta introducción, se resume la metodología empleada. A continuación en el tercer epígrafe se describe la pasarela estudiada, los parámetros modales que identifican su comportamiento dinámico y los procesos de modelización y ajuste de la estructura. En el cuarto apartado se presentan ejemplos de la respuesta dinámica simulada, y se comparan con la registrada experimentalmente para finalmente presentar las principales conclusiones que se deducen de este trabajo.

\section{Metodología}

\subsection{Modelización y ajuste}

La metodologra seguida en el presente trabajo ha consistido en primer lugar en la modelización de la pasarela, partiendo de la geometría de proyecto, mediante el programa de elementos finitos ANSYS y procediendo posteriormente a su ajuste empleando para ello un esquema iterativo que permitió analizar y seleccionar aquellos parámetros f́sicos cuyo efecto era más relevante para tál fin. Respecto al ajuste del modelo mecánico (conocido como FEM Updating en la terminologra inglesa), varios autores [8-15] han fin. Respecto al ajuste del modelo mecánico (conocido como FEM Updating en la terminologia inglesa), varios autores [8-15] han
publicado y comparado varias técnicas sin alcanzarse ningún acuerdo sobre la existencia de un procedimiento general que pueda publicado y comparado varias técnicas sin alcanzarse ningún acuerdo sobre la existencia de un pros
aplicarse a todos los casos, por lo que la elección ha dependido de los parámetros seleccionados.

Ésta se ha realizado en función de los parámetros modales extrafdos de las campańas experimentales, empleando como referencia las frecuencias y las formas modales en el caso de la pasarela completa y complementándolo con las masas modales y/o modos nomalizados respecto a la matriz de masa.

Dentro de los métodos iterativos de ajuste, se ha utilizado una aproximación para correlacionar el modelo experimental y el modelo matemático que consiste en la comparación entre las frecuencias naturales [16], pudiéndose la diferencia en porcentaje $\left(\epsilon_{f}\right)$ expresarse tal y como se muestra a continuación:

$$
\varepsilon_{f t}=\frac{\mid f_{A t}-f_{X I} \perp}{f_{A t}} \times 100
$$

siendo $f_{X t}$ el parámetro experimental y $f_{A t}$ el parámetro calculado.

Para medir la correlación entre los modos normalizados respecto a la matriz de masa experimentales y los obtenidos mediante el modelo en ANSYS se ha empleado el criterio de confianza modal (MAC según la abreviatura de su denominación en inglés) que proporciona una medida del grado de linealidad entre estimaciones de los modos de vibración originados desde diferentes fuentes [16]. Se define como un escalar y su expresión se muestra a continuación:

$$
M A C_{t j}=\frac{\left|\left\{\Psi_{A}\right\}_{t}^{H}\left\{\Psi_{X}\right\}_{j}\right|^{2}}{\left(\left\{\Psi_{A}\right\}^{H}\left\{\Psi_{A}\right\}_{t}\right)\left(\left\{\Psi_{X}\right\}^{H}\left\{\Psi_{X}\right\}_{j}\right)}
$$

siendo $\Psi_{X}$ el modo de vibración experimental y $\Psi_{A}$ el modo de vibración calculado, donde la notación $f^{X}$ significa transpuesta compleja conjugada (matriz hermítica).

\subsection{Simulación de cargas}

Una vez ajustado el modelo se ha estudiado el comportamiento del mismo ante dos escenarios reales diferentes. El primero de ellos 
ha consistido en la simulación de las fuerzas generadas durante el EMA realizado en el vano más susceptible a las vibraciones (vano 2). En el segundo supuesto se ha simulado el tránsito peatonal a lo largo de toda la pasarela. En ambos casos el objetivo es obtener la respuesta dinámica en forma de aceleraciones del modelo numérico y poder comparar los resultados con los registrados experimentalmente con el fin de validarlo.

Para simular el efecto de varios peatones (solicitación del segundo escenario estudiado) se ha empleado el modelo convencional que considera que la respuesta de un flujo de peatones (sin ninguna sincronización particular) se obtiene multiplicando el efecto de un solo peatón por un factor igual a $\sqrt{N}$, siendo $\mathrm{N}$ el número de peatones presentes en la pasarela en un momento determinado [1]. La velocidad del peatón se ha considerado igual a 0.9 veces la frecuencia de paso en $\mathrm{Hz}$.

La fuerza de excitación generada por un solo peatón (movimiento rítmico al caminar) se ha expresado matemáticamente mediante una serie de Fourier de la forma:

$$
F(t)=G_{0}+G_{1} \sin 2 \pi f_{m} t+\sum_{t=2}^{n} G_{t} \sin \left(2 \pi i f_{m} t-\varphi_{t}\right)
$$

donde

$G_{0}=$ fuerza estática (componente vertical del peso de un peatón)

$G_{1}=$ amplitud del primer armónico

$G_{l}=$ amplitud del i-ésimo armónico

$f_{m}=$ Frecuencia o cadencia de la actividad expresada en Herzios $(\mathrm{Hz})$.

$\varphi_{t}=$ ángulo de desfase del i-ésimo armónico respecto al primero

$n=$ número de armónicos

En el caso que estamos analizando, consideramos la actividad de caminar/marcha (contacto continuo con el suelo), estableciendo una frecuencia de paso en torno a $1.8 \mathrm{~Hz}$.

Se ha estimado que con $n=3$ se obtiene una precisión adecuada, siendo los valores de los coeficientes los siguientes: $G_{0}=700 \mathrm{~N}$, $G_{1}=0,4 G_{0}, G_{2}=G_{3} \approx 0,1 G_{0}, \varphi_{2}=\varphi_{3} \approx \pi / 2$

\section{Resultados numéricos y discusión}

\subsection{Resultados del análisis modal experimental}

La Pasarela del Museo de la Ciencia es una estructura en servicio situada en Valladolid sobre el río Pisuerga (figura 1). Fu in augurada en 2004 y tiene una longitud total de 234 metros repartidos en cuatro vanos: tres formados por barras metálicas de 51 , 111 y 21 metros de luz (vano 2, 3 y 4 respectivamente), y uno de hormigón de 45 metros (vano 1). Éste último es muy rígido por lo que no se considera en el análisis objeto del presente trabajo. La tipología resistente de los vanos metálicos es de entramado triangulado de barras tubulares con sección pretensado formado por un conjunto de cables longitudinales y transversales en el vano 3 (figuras 2 y 3 ).

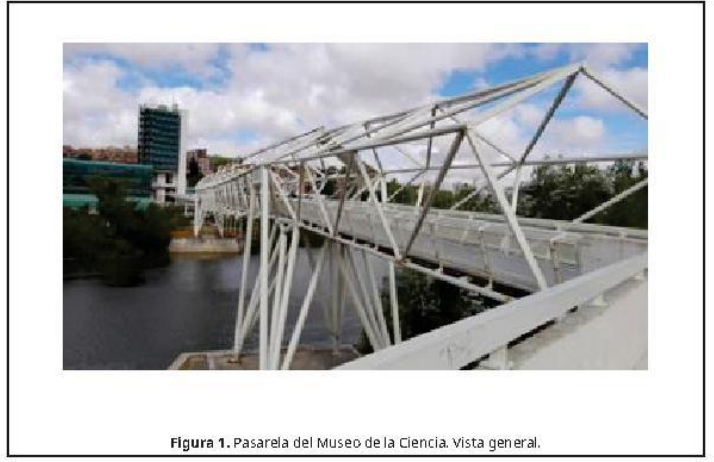




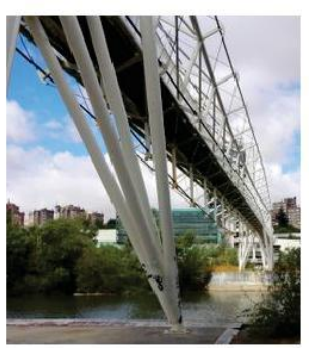

Figura 2. Pasarela del Museo de la Ciencia. vista del vano 3.

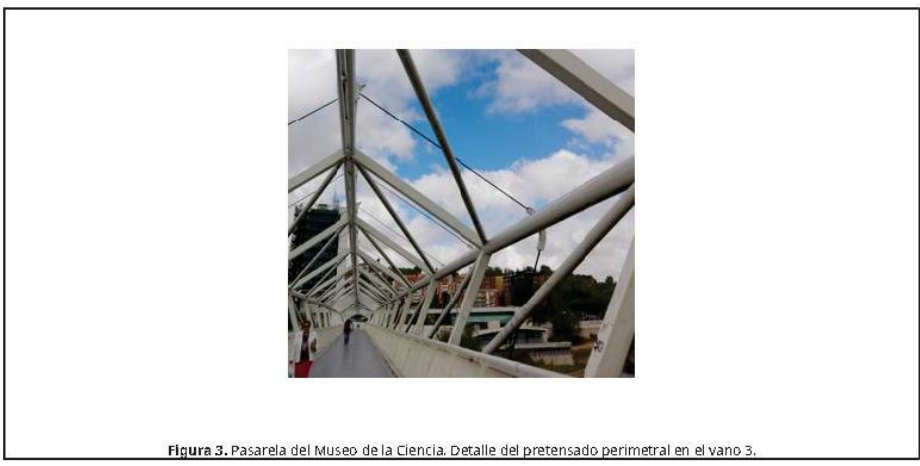

En el trabajo de investigación llevado a cabo por J. de Sebastián Sanz [5] se monitorizaron durante varios meses las aceleraciones verticales y laterales en el centro de cada uno de los tres vanos metálicos de la pasarela observándose que los niveles de vibración en la dirección lateral eran considerablemente menores que en la dirección vertical y que el nivel de vibraciones del vano 2 era notablemente mayor que el del vano 3. Más adelante se identificaron mediante un OMA los parámetros dinámicos de la estructura a partir de los registros de aceleración en dirección vertical monitorizados a lo largo de los tres vanos metálicos de la pasarela. Dichos parámetros modales se obtuvieron mediante el programa comercial ARTeMIS empleando tres algoritmos en el dominio de la frecuencia (FDD (Frequency Domain Decomposition), EFDD (Enhanced Frequency Domain Decomposition) y CFDD (Curve-Fit Frequency Domain Decomposition)] [17-19]. Éstos se recogen en la tabla 1 para los primeros cuatro modos de vibración:

$$
\begin{aligned}
& \text { Tabla 1. Frecuencias identificadas experimentalmente. } \\
& \text { FDD CFDD CFDD }
\end{aligned}
$$

Frecuencia $(\mathrm{Hz})$ Frecuencia $(\mathrm{Hz})$ Frecuencia $(\mathrm{Hz})$

$\begin{array}{lll}1.245 & 1.243 & 1.257 \\ 2.759 & 2.763 & 2.765 \\ 3.516 & 3.514 & 3.515 \\ 4.785 & 4.792 & 4.792\end{array}$

Los cuatro primeros modos de flexión de la pasarela completa se presentan a continuación. 


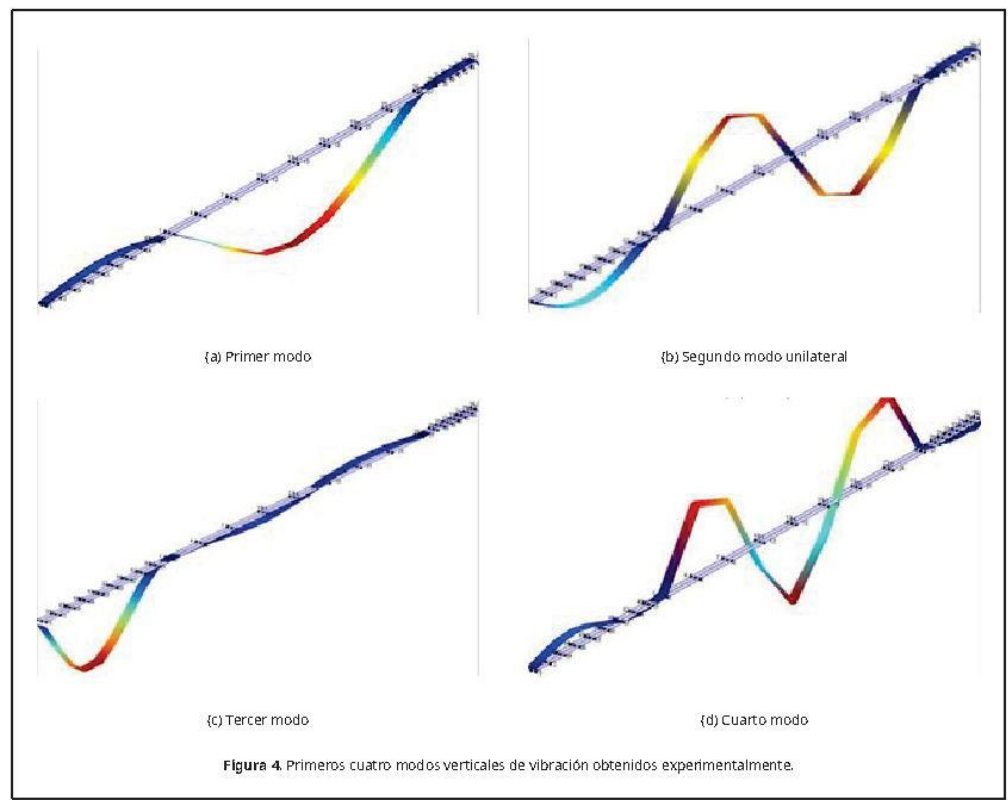

Debido a la mayor susceptibilidad del vano 2 de la pasarela, se realizó un EMA en el mismo identificando, entre otros, la masa moda vertical mediante el programa comercial OROS Modal 2. Se realizaron asimismo en este mismo estudio varias estimaciones de dich masa modal empleando diferentes algoritmos, presentándose en la tabla 2 los resultados obtenidos [5].

$\begin{array}{lc}\text { Tabla 2. Estimaciones de la masa modal del modo vertical } 3 . \\ \text { Método } & \text { Masa Modal (kg) } \\ \text { Programa OROS } & 16983 \\ \text { Algoritmo en frecuencia } & 17950 \\ \text { Algoritmo en el tiempo } & 18060 \\ \text { Ajuste de la FRF } & 17900 \\ \text { Aumento de la respuesta } & 17500\end{array}$

Posteriormente empleando los registros del EMA realizado en el vano 2 se calcularon las masas modales y los modos nomalizados asociados a cada modo de vibración de dicho vano mediante dos técnicas de escalado. Ambas parten de la información obtenida al aplicar el método de identificación de subespacios estocásticos, en inglés Stochastic Subspace Identification (SSI), consistiendo la primera de ellas en la resolución de un problema simétrico de valores propios y la segunda en el escalado de los modos de vibración a partir de la estimación de los residuos de la descomposición en fracciones parciales de la función de respuesta en frecuencia (FRF) de las mediciones [6.7 Aplicando ambas técnicas se calcularon las masas modales y los valores de los modos normalizados respecto a la matriz de masa en las coordenadas $L / 3, L / 2$ y $2 L / 3$ del vano $2(L=51 \mathrm{~m})$

En la tabla 3 se indican los modos de vibración nomalizados y la masa modal del modo vertical 3 de la pasarela estimado mediante el problema simétrico de valores propios expresado en el espacio de estados (método 1) [6].

$$
\begin{aligned}
& \begin{array}{l}
\text { Tabla 3. Modos de vibración normalizados y masa modal del modo vertical } 3 \text { de la pasarela completa \{según método 1\}. } \\
\text { Masa modal }(\mathbf{k g})
\end{array} \\
& \text { L/3 } \\
& \mathrm{L} / 2 \\
& \begin{array}{llll}
17605.0 & 0.0039 & 0.0075 & 0.0058
\end{array}
\end{aligned}
$$

Valor medio

Desviación típica 
Valor medio

Desviación típica
17814.9

952.9
0.00390 .00750 .0045

$0.00010 .0002 \quad 0.0001$

En la tabla 4 se indican los modos de vibración nomalizados y la masa modal del modo vertical 3 de la pasarela mediante escalado a partir de la estimación de los residuos de la descomposición en fracciones parciales de la Función de Respuesta en Frecuencia (FRF) de las mediciones (método 2) [7].

\subsection{Generación del modelo de la pasarela}

La estructura del tramo metálico de la pasarela (vanos 2, 3 y 4) consiste en una celosía tubular tridimensional de sección transversal hexagonal (figura 5), que en el vano 3 se sitúa en el interior de otra celosía formada por cables pretensados longitudinales y transversales. Ambas celosias se enlazan mediante radios tubulares que permiten mantener la forma de los cables y aumentar su excentricidad en el centro del vano. La sección transversal es un hexágono irregular cuya inclinación y dimensiones cambian a lo
largo del puente [20].

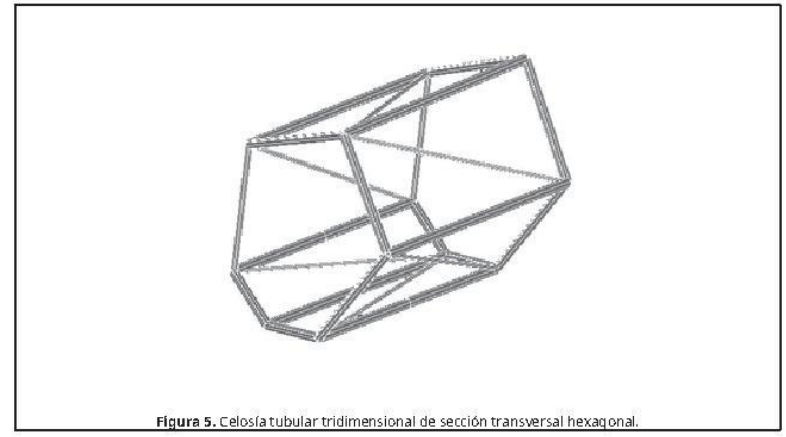

En cuanto al pretensado transversal, cada sección hexagonal del vano 3 (son 15) consta de un tendón periférico en forma de hexágono, cuyos vértices son los extremos de los seis radios. El pretensado longitudinal consta de seis tendones que pasan por los extremos de los radios de las secciones transversales [21]. Se ha modelizado la estructura de forma que los nudos que conforman tanto el hexágono exterior como el interior, sean coplanarios (figura 6 ).

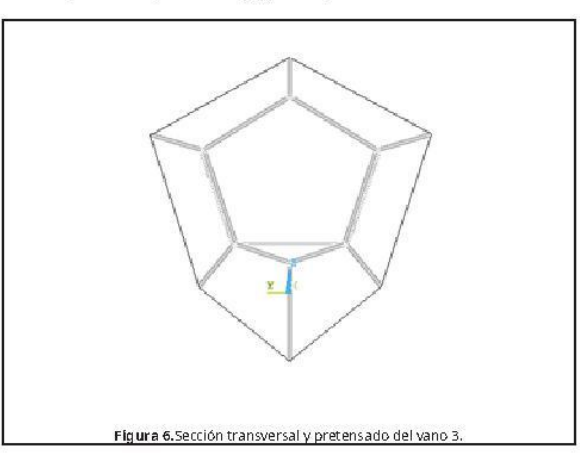

La estructura principal está formada por perfiles tubulares de sección circular (figura 7). El diámetro de los perfiles longitudinales es de $273 \mathrm{~mm}$ y las diagonales son perfiles de diámetro $135 \mathrm{~mm}$. Los montantes y radios son perfiles tubulares de sección cuadrada de dimensiones $200 \mathrm{~mm} \times 200 \mathrm{~mm}$. 


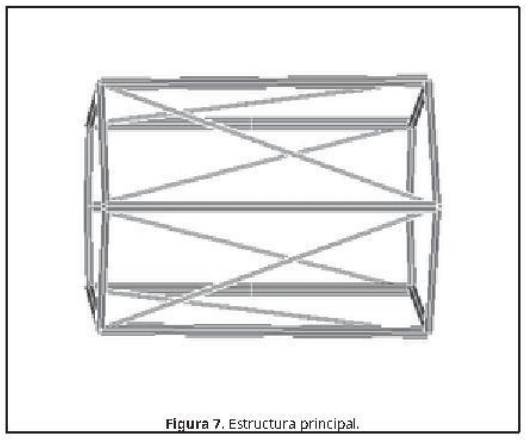

Las secciones de la estructura del tablero (figura 8), constituida por travesanos y arriostramientos, son cuadradas de dimensiones $120 \mathrm{~mm} \times 100 \mathrm{~mm}$ y $120 \mathrm{~mm} \times 120 \mathrm{~mm}$ respectivamente.

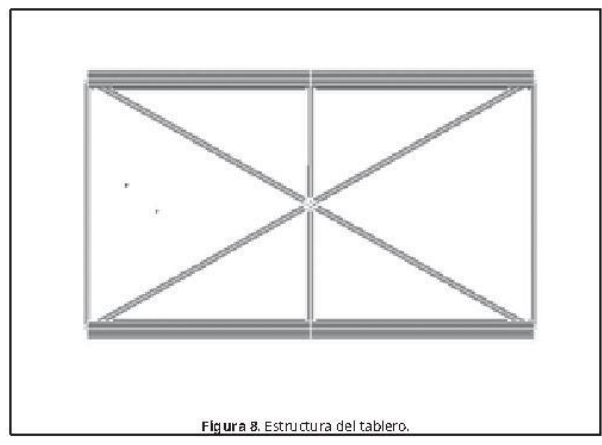

Los apoyos centrales son dos torres constituidas por celosías resueltas también con perfiles tubulares de 355 mm de diámetro (figura 9).

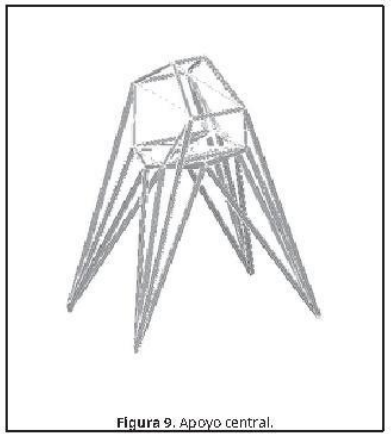

En cuanto a las propiedades del acero se ha considerado un módulo de elasticidad de $2,1 \cdot 10^{11} \mathrm{~N} / \mathrm{m}^{2}$, un coeficiente de Poisson igual a 0,3 y una densidad de $7.700 \mathrm{~kg} / \mathrm{m}^{3}$.

La modelización de la estructura se ha realizado mediante el programa ANSYS empleando dos tipos de elementos: BEAM188 para simular los perfiles tubulares, definiendo para cada tipo las secciones indicadas anteriormente y LINK180 para los tendones de pretensado (figura 10). En el caso del pretensado longitudinal se ha impuesto que los movimientos en la dirección lateral y vertical de los nudos que lo modelizan sean iguales a los de los nudos que representan los extremos de los radios de las secciones transversales. La fuerza de pretensado se ha modelizado mediante el comando INISTATE. 
La principal dificultad en la definición geométrica de la pasarela ha sido la modelización del pretensado, principalmente por la elección del tipo de elemento y de las conexiones de éste con el resto de la estructura con el fin de que la transmisión de esfuerzos se realizara de forma realista.

En cuanto a los apoyos, la estructura se considera apoyada en las torres centrales y empotrada en los extremos del tablero. Las bases de las torres se suponen empotradas.

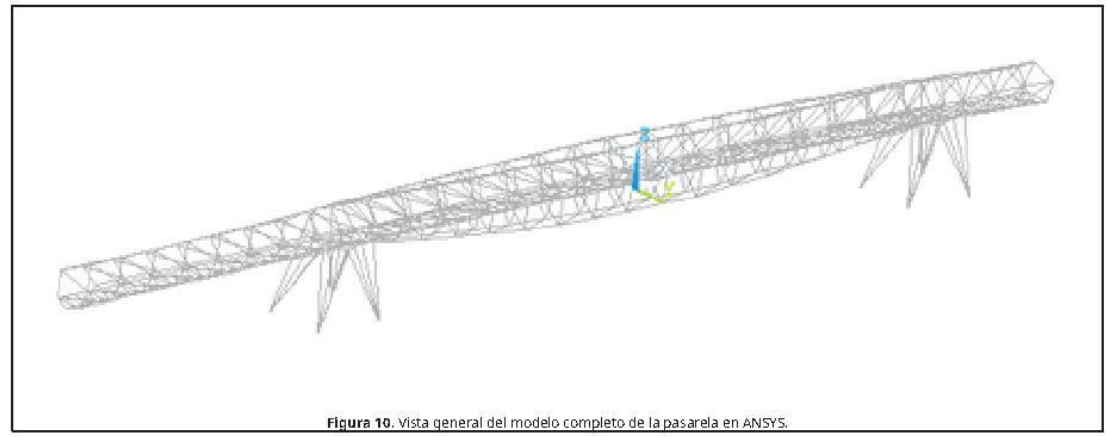

3.3. Ajuste del modelo de la pasarela

Para el ajuste del modelo de la pasarela se emplearon todos los datos experimentales expuestos en los estudios anteriores [5] que afectaban a los cuatro primeros modos verticales de vibración de la pasarela completa, esto es, las frecuencias y las formas modales de dichos modos y la masa modal y los modos nomalizados del tercer modo de vibración.

Se empleó un esquema iterativo que nos permitió analizar y seleccionar aquellos parámetros físicos cuyo efecto era más relevante para que las frecuencias y las formas modales de nuestro modelo se aproximaran lo más posible a las obtenidas experimentalmente. En nuestro caso se comprobó que la masa era la variable que mayor influencia tenfan en el ajuste una vez modelizada la geometría de la estructura.

En el caso de la masa, ha sido la principal variable que ha afectado al ajuste del tercer modo vertical, no sólo de la frecuencia y la forma modal sino también de su masa modal.

Se ha seleccionado para el análisis modal realizado el método de Block Lanczos y se han obtenido los primeros cuatro modos verticales, que a continuación se reflejan.

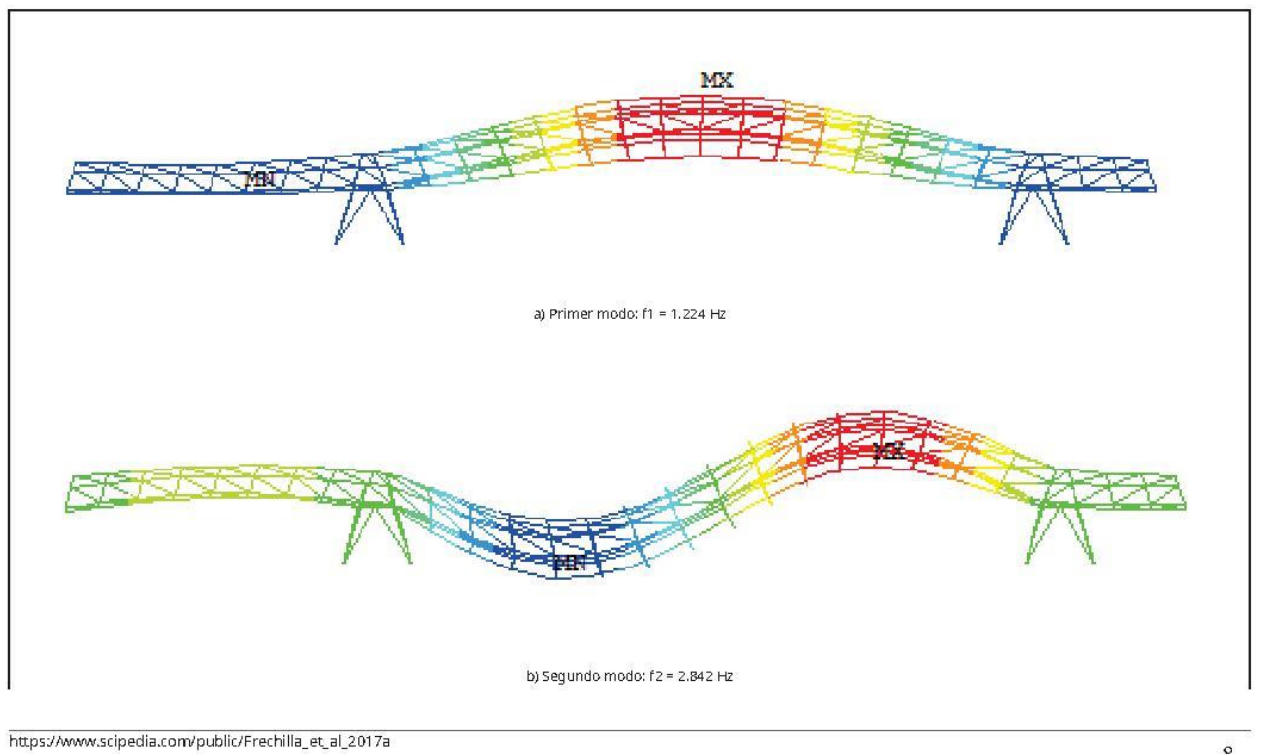



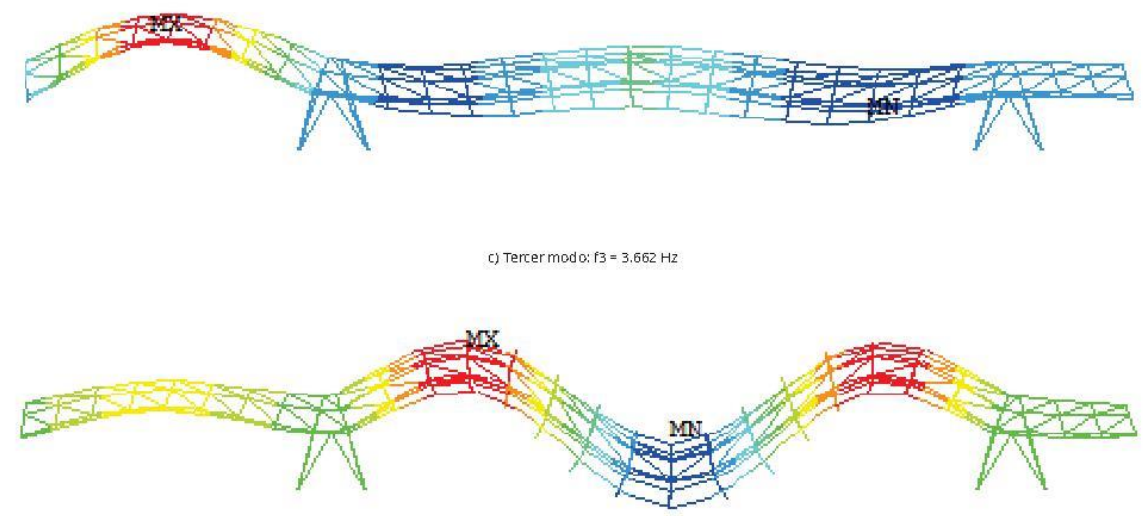

d) Cuarto $\operatorname{modo}: f_{4}=4.914 \mathrm{~Hz}$

Figura 11. Modos verticales obtenidos con el modelo en ANSYS

Tal y como puede observarse la correspondencia entre las formas modales del modelo en ANSYS (figura 11) y las del OMA (figura 4) es buena.

En la tabla 5 se comparan los resultados del modelo numérico con los obtenidos experimentalmente (tabla 1).

Tabla 5. Comparativa entre las frecuencias obtenidas experimentalmente y las obtenidas mediante la modelización de la pasarela en ANSY5.

$\begin{array}{ccccc}\text { Modo } & f_{M E F} & \left|f_{M E F}-f_{F D D}\right| / f_{M E F} & \left|f_{M E F}-f_{E F D D}\right| / f_{M E F} & \left|f_{M E F}-f_{C F D D}\right| / f_{M E F} \\ & (H z) & (\%) & (\%) & (\%) \\ 1 & 1.224 & 1.72 & 1.55 & 2.70 \\ 2 & 2.842 & 2.92 & 2.78 & 2.71 \\ 3 & 3.662 & 3.99 & 4.04 & 4.01 \\ 4 & 4.914 & 2.63 & 2.48 & 2.48\end{array}$

En todos los casos se obtienen diferencias menores del $5 \%$ lo que indica una buena correspondencia del modelo.

En el caso de la masa modal del tercer modo se ajustó a un valor de $18057 \mathrm{~kg}$ que corresponde aproximadamente al valor máximo que se ha obtenido en las estimaciones experimentales (tablas 2, 3 y 4). La diferencia en porcentaje con respecto al resto de las estimaciones no supera el $6 \%$

En la tabla 6 se indican los valores resultantes de la comparación entre los modos normalizados respecto a la matriz de masa del modelo en ANSYS y los experimentales obtenidos en $\underline{[6,7]}$.

Tabla 6. Valores de MAC comparando los modos normalizad os obtenid os numéricamente ylos experimentales empleando técnicas de escalado.

Técnica de obtención de los modos nomalizados

$$
\text { L/3 }
$$

Modelo ANSYS

Método 1

Método 2
Coordenadas MAC $\mathrm{L} / 2 \quad 2 \mathrm{~L} / 3$ $\begin{array}{lll}0.0069 & 0.0074 & 0.0055\end{array}$ $\begin{array}{llll}0.0039 & 0.0075 & 0.0058 & 0.938\end{array}$ $\begin{array}{llll}0.0039 & 0.0075 & 0.0045 & 0.944\end{array}$

Los resultados son cercanos a la unidad lo que nos indica una buena correspondencia entre modos.

\section{Análisis dinámico y validación del modelo}


Una vez ajustado el modelo de elementos finitos de la pasarela, se obtienen los modos normalizados de los cuatro primeros modos de vibración y, con el resto de los parámetros modales, se procede a simular diferentes fuerzas de excitación para obtener su respuesta, en forma de aceleraciones. El objetivo es poder comparar los resultados con los obtenidos experimentalmente con el objetivo de validar la modelización. Para obtener la respuesta dinámica del modelo se emplearon los valores medios de los coeficientes de amortiguamiento obtenidos experimentalmente en [5] correspondientes a dichos modos. Estos coeficientes se muestran en la tabla 7.

$\begin{array}{ccc}\text { Tabla 7. Coeficientes de amortiguamiento identificados experimentalmente. } & \\ \text { Modo } & \text { Amortiguamiento Método EFDD (\%) } & \text { Amortiguamiento Método CFDD (\%) } \\ 1 & 1.173 & 1.233 \\ 2 & 0.563 & 0.914 \\ 3 & 0.447 & 0.436 \\ 4 & 0.345 & 0.327\end{array}$

Para validar el comportamiento dinámico observado en el vano 2 del modelo se simularon las fuerzas generadas durante el EMA y se calcularon las aceleraciones en los mismos puntos en los que se obtuvieron mediciones experimentales $(L / 3, L / 2$ y $2 \mathrm{~L} / 3$ del vano 2 siendo $L=51$ ). A continuación, se muestran algunos ejemplos de fuerzas simuladas (figura 12) y de las comparativas entre las respuestas experimentales y las obtenidas numéricamente (figuras 13, 14y 15).
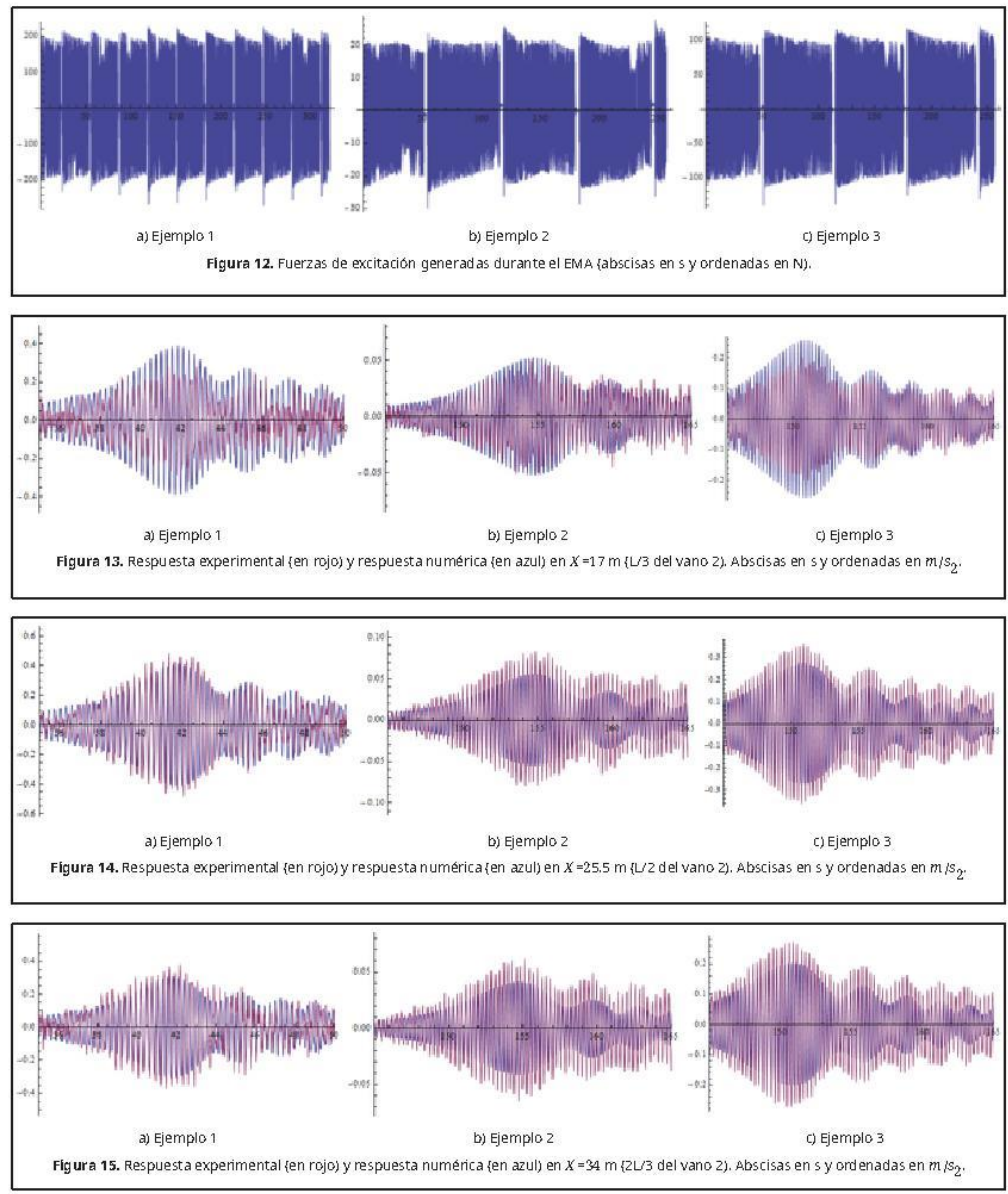
Tabla 8. valores de aceleraciones máximas $\{e n$

$\begin{array}{cccc}\text { Prueba } & \text { Cota } & \text { Numérica } & \text { Experimental } \\ 1 & L / 3 & 0,389 & 0,290 \\ L / 2 & 0,413 & 0,486 & 14,9 \\ 2 \mathrm{~L} / 3 & 0,308 & 0,380 & 19,1 \\ 2 & L / 3 & 0,052 & 0,050 \\ \mathrm{~L} / 2 & 0,056 & 0,083 & 32,7 \\ 2 \mathrm{~L} / 3 & 0,041 & 0,062 & 33,2 \\ 3 & L / 3 & 0,257 & 0,200 \\ \mathrm{~L} / 2 & 0,273 & 0,359 & 24,0 \\ 2 \mathrm{~L} / 3 & 0,203 & 0,271 & 25,1\end{array}$

\section{Diferencia (\%)}

25,6

4,8

22,2

Como puede observarse en la tabla 8 , donde se muestran las aceleraciones máximas obtenidas tanto numérica como experimentalmente, las respuestas del modelo son ligeramente inferiores salvo a $L / 3$ del vano 2, que son algo superiores debido a que el modo normalizado del modelo en esa coordenada es también superior al obtenido experimentalmente. En el resto de los casos las diferencias son probablemente consecuencia de que el modelo sólo tiene en cuenta la fuerza de excitación aplicada y no el resto de las cargas ambientales que pudieran existir. Las diferencias son más notables y variables en el caso de que la fuerza artificial aplicada es menor y por tanto el peso en la respuesta de las cargas ambientales es mayor, lo que corroborarfa la hipótesis anterior. Tal y como queda reflejado en la tabla anterior (tabla 8) las diferencias en el valor máximo de las aceleraciones se encuentran en torno al $25 \%$ lo que indica que la correspondencia entre la respuesta del modelo y la experimental es buena.

Para estudiar el comportamiento del modelo completo se ha procedido a simular la principal solicitación a la que están sometidas este tipo de estructuras, esto es, el tránsito peatonal, para comparar la respuesta obtenida con la registrada experimentalmente en varios puntos de la pasarela y así verificar el comportamiento de la estructura completa.

Las aceleraciones que se emplean de referencia se extraen de registros de aceleraciones verticales que corresponden al tránsito peatonal de hasta 10 individuos! .

${ }^{1}$ La influencia de la masa de los peatones en el factor de amortiguamiento del modelo de la estructura no se tiene en cuenta puesto que representa un valor menor al $5 \%$ de la masa total de
la pasarela [22].

La simulación de dicha solicitación sobre la pasarela se realiza empleando el programa Mathematica y se obtienen las respuestas dinámicas en aquellos puntos donde tenemos registros experimentales para poder comparar posteriormente ambos resultados.

A continuación se muestran algunos ejemplos de las comparativas obtenidas entre respuestas dinámicas en forma de aceleraciones del modelo numérico y las registradas experimentalmente (figuras 16,17 y 18 ):
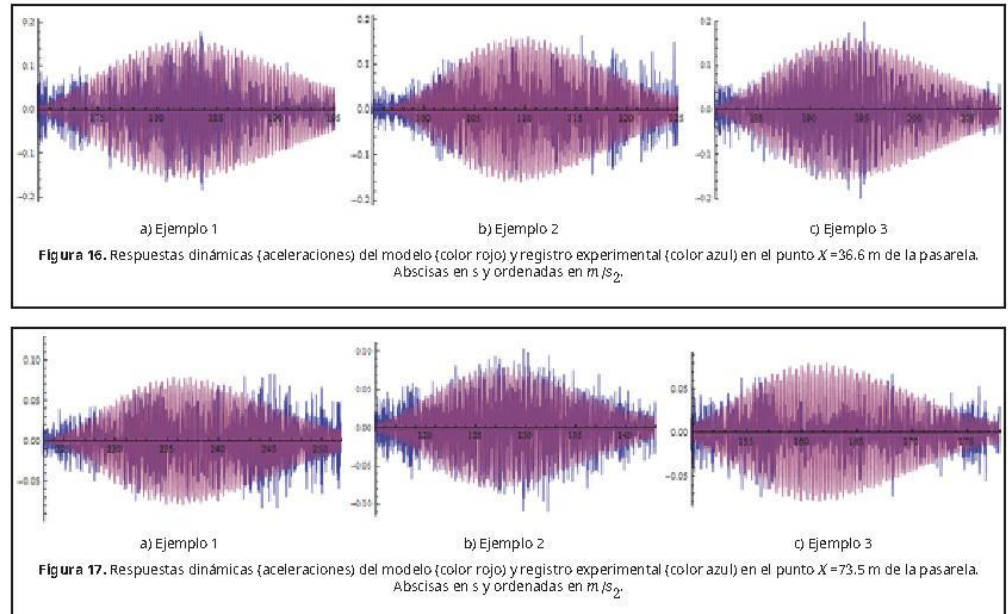


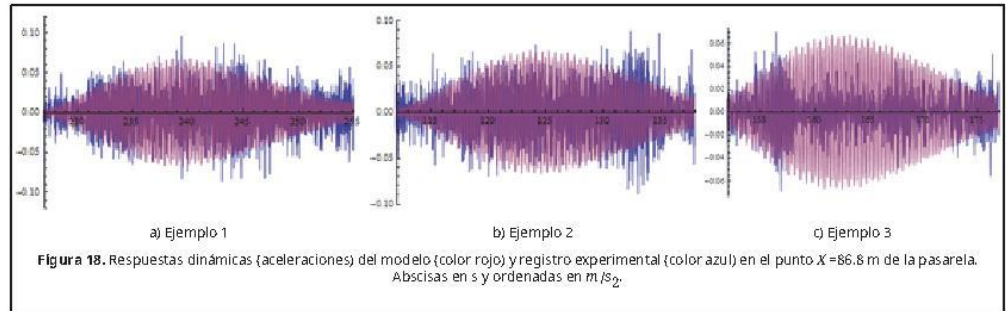

Siendo conscientes de las limitaciones del modelo de flujo peatonal y de la variabilidad de los registros experimentales de este tipo de aceleraciones, la finalidad del análisis de estos resultados es comprobar que los obtenidos numéricamente son del mismo orden de magnitud que los reales y que la estructura modelizada completa simula con adecuada precisión este tipo de solicitación. Tal y como puede desprenderse de las figuras anteriores (figuras 16,17 y 18) los valores máximos alcanzados analíticamente son similare a los experimentales, situándose las mayores diferencias en torno al $20 \%$ en la mayorfa de los casos.

\section{Conclusiones}

A la vista de los resultados obtenidos se puede llegar a la conclusión de que el modelo de elementos finitos representa de forma bastante precisa los parámetros modales de la estructura a pesar de la complejidad geométrica de la misma.

En el caso de la respuesta dinámica se obtienen buenos resultados considerando la multitud de factores -y errores inherentes a ellos- que intervienen tanto en la obtención de los resultados numéricos como los experimentales ten especial en el caso del tránsito peatonal). Se ha podido comprobar que si la geometrfa de partida es realista y completa el ajuste únicamente de las frecuencias y formas modales proporciona una precisión adecuada del modelo.

Una vez validado, este modelo nos permitirá estudiar los diferentes factores que provocan la susceptibilidad a las vibraciones de la pasarela para, por ejemplo, contemplar diferentes alternativas de diseño y/o predecir su comportamiento ante diferentes solicitaciones durante las diversas fases del Proyecto. Asimismo se puede emplear el conocimiento generado en el presente estudio en las fases de diseno de otros casos similares, reduciendo de este modo las inversiones que serían necesarias implantar para solucionar efectos dinámicos no deseados en etapas más tardías que habitualmente son mucho más elevadas. Este análisis puede abrir nuevas líneas de investigación que pueden ser recogidas en futuras publicaciones.

Todo ello contribuirá a una mejor compresión del comportamiento dinámico de este tipo de estructuras favoreciendo asimismo la proliferación del uso de este tipo de herramientas y asi ayudar a los proyectistas y a las administraciones responsables de su diseno y mantenimiento.

\section{Agradecimientos}

Este trabajo ha sido realizado en parte con la financiación aportada al proyecto de investigación BIA2011-28493-C02-02 por el programa de apoyo a Proyectos de Investigación Fundamental No Orientada (PIFNO) del Ministerio de Ciencia.

Los autores agradecen al Ayuntamiento de Valladolidy al Museo de la Ciencia su apoyo en las pruebas de campo.

\section{Bibliografía}

[1] P. Charles, W. Hoorpah, Technical guide - footbridges - Assessment of vibrational behavior of footbridges under pedestrian loading, Service d'Etudes Techniques des Routes et Autoroutes (SETRA), Paris, 2006.

[2] FIB. Bulletin 32. Guidelines for the design of footbridges. Fédération internationale du beton (FIB), 2005.

[3] M. Feldmann, C. Heinemeyer, M. Lukic, Design of Footbridges. Guideline. Human Induced vibrations of Steel Structure (Hivoss), Hivoss-RFS2-CT-2007-00033, 2008.

[4] J. E. Mottershead, M.I. Friswell, Model updating in structural dynamics: A survey, Journal of Sounds and vibration. 167(2) (1993) 347-375.

[5] j. De Sebastián, Análisis del Estado Límite de Servicio y Control de Vibraciones en pasarelas peatonales, Tesis Doctoral, Universidad de Valladolid, 2014.

[6] M. Cacho-Pérez, N. Frechilla, A. Lorenzana, Estimación de las masas modales de una estructura en servicio mediante transformación en el espacio de estados, Revistá Internacional de Métodos Numéricos para Cálculo y Diseno en Ingenier(a. 33(1) (2017) 123-128. DOI: http://dx.doi.org/10.1016/j.rimni.2016.02.002

[7] M. Cacho-Pérez, N. Frechilla, A. Lorenzana, Estimación de parámetros modales de estructuras civiles a partir de la función de respuesta en frecuencia, Revista Internacional de Métodos Numéricos para Cálculo y Diseño en Ingenieria. (2016). DOI: http://dx.doi.org/10.1016/j.rimni.2015.02.001. 
[8] B. Caesar, Updating system matrices using modal test data, Procedings of the 5 th International Modal Analysis Conference, London. (1987) 453-459.

[9] S. R. Ibrahim, A.A. Saafan, Correlation of analysis and test in modelling of structures assessment and review. Procedings of the 5th International Modal Analysis Conference, London. (1987) 1651-1660.

[10] W. Heylen, P. Sas, Review of model optimisation techniques, Procedings of the 5th International Modal Analysis Conference, London. (1987) 1177-1182

[11] H. G. Natke, Updating computational models in the frequency domain based on measured data: A Survey, Probabilistic Engineering Mechanics. 4 (1988) 28-35.

[12] M. Imregun, W.J. Visser, A review of model updating techniques, The Shock and Vibration Digest. 23(1)(1991) 9-20.

[13] J.E.Mottershead, M.I. Friswell, Model updating in structural dynamics: A survey, Journal of Sounds and vibration. 167(2) (1993) 347-375.

[14] M.I. Friswell, ].E. Mottershead, Finite Element Model Updating in Structural Dynamics, Kluwer Academic publishers, 1995.

[15] W. Vélez, D. Gómez, P. Thomson, Ajuste de Modelos de Elementos Finitos, Dyna. 76(158) (2009) 177-189.

[16] S. Ziaei Rad, Methods for Updating Numerical Models in Structural Dynamics, Tesis, University of London, 1997.

[17] R. Brincker, L. zhang. P. Andersen, (2000, February). Modal identification from ambient responses using frequency domain decomposition, Proceedings. of the 18 International Modal Analysis Conference (IMAC), San Antonio, Texas. (2000).

[18] S. Gade, N. Møller, H. Herlufsen, H. Konstantin-Hansen, Frequency domain techniques for operational modal analysis, ist IOMAC Conference (2005)

[19] N.J. Jacobsen, P. Andersen, R. Brincker, Applications of frequency domain curve-fitting in the EFDD technique, Proceedings IMAC XXVI Conference. (2008)

[20] ]. Martínez Calzón, R. Moneo Vallés, E. De Teresa Trilla, (2007) Pasarela del Museo de la Ciencia sobre el río Pisuerga (Valladolid) Boletín Informativo Construber. 8 (2007) 10-13

[21] P. Ladret, A. González, Pretensado exterior en la pasarela del Museo de la Ciencia en Valladolid, Informes de la Construcción, 57(497) (2005).

[22] C. Maraveas, Z.C. Fasoulakis and K.D: Tsavdaridis, A review of Human Induced vibrations on Footbridges, American Journal of Engineering and Applied Sciences. 8(4) (2015) 422-433. 



\section{ANEXO. OTRAS PUBLICACIONES}


"Determination of the Bending Strength Geometry Factor J for the Calculation of Stress in Parallel-Axis Gears as a Function of the Reference Correction and Slope Angle of the Gear Tooth"

Noelia Frechilla Alonso, Roberto José Garcia Martin and Pablo Frechilla Fernández

International Journal of Mechanical Engineering and Automation. Volume 3, Number 1, 2016, pp. 27-33. 


\title{
Determination of the Bending Strength Geometry Factor
}

$\mathrm{J}$ for the Calculation of Stress in Parallel-Axis Gears as a

\section{Function of the Reference Correction and Slope Angle of the Gear Tooth}

\author{
Noelia Frechilla Alonso, Roberto José Garcia Martin and Pablo Frechilla Fernández \\ Mechanical Engineering Department, University of Salamanca, Zamora 49022, Spain \\ Corr esponding author: Roberto José Garcia Martin (toles@usal.es)
}

Abstract: In this paper, the authors report a new method for calculating the bending strength geometry factor defined in norm 908-B89 of AGMA (American Gear Manufacturers Association), which is used for the determination of the bending forces at the base of IS (International Standard) - specified gear teeth, represented as $J$. Via complex algorithms, this norm calculates the coefficient, taking into account the number of teeth on the gear/pinion and of the wheel, the pressure angle of the tooth and the angle of the tooth helix and the correction or displacement of the tool with respect to the standard pitch radius. The aim of this work is to establish a general formulation that is easy to use for the determination of the bending strength geometry factor (henceforth "form coefficient") for both straight-toothed gears and helical-toothed gears for zero-corrected gears and "V" gears.

Keywor ds: Form factor, cog correction, finite elements method, mathematical model.

\section{Introduction}

The current development of mechanical engineering means that gears are one of the most efficient ways for the transmission of motion with reduced spaces between the shafts since they have a large load capacity, the angular velocity ratio remains constant through the coupling, and the materials employed support greater work bending strength. This means that the study of these issues is even further ahead of the advances in modern industry.

Examples of the importance of these elements can be found in the many historical references to the use of mechanisms similar to those familiar today (gears). Outstanding among these are the Mechanism of Aristotle, described in the book on mechanisms $(280$ $\mathrm{CE}$ ), the Carriage Looking Southwards (China 120-250 AC), etc.
On addressing this work, we have attempted to find answers to the following questions, which arose in Refs. [1-3] and which should allow us to separate the original problem issues, allowing the research to follow a concrete direction:

- What is the influence of the correction of the teeth and of the angle of the helix on the geometric factor of the gear?

- How do the above factors influence the bending strength at the base of a gear wheel qualitatively and quantitatively?

- What differences are there between the forces at the base of the teeth obtained with the finite element model and those defined in the AGMA-IS norm?

In this sense, the present work attempts to offer results of the bending strength geometry factor $\mathrm{J}$, where the scientific novelty is seen in the generation of a value of a single form coefficient that can be used 
28 Determination of the Bending Strength Geometry Factor $\mathrm{J}$ for the Calculation of Stress in Parallel-Axis Gears as a Function of the Reference Correction and Slope Angle of the Gear Tooth

to calculate the forces at the base of the teeth, regardless of whether they are straight, helical or with or without an addendum modification coefficient.

The procedure followed was as follows: use of $\mathrm{CAD}[4]$ and $\mathrm{CAE}$ tools, which include calculation by FEM (finite elements method) to obtain the bending strength at the base of the gear tooth $[5,6]$ in order to assess the form factor.

With the results the variation in that factor with the displacement of the tooth and the reference slope angle of the dentate (Fig. 1), also known as the helix angle, was plotted graphically.

We have developed a mathematical formulation $(\mathrm{Eq}$ (14)), based on the results obtained from the simulations carried out, able to modify the form factor of the AGMA to include the effect of the addendum modification coefficient and that of the slope angle of the gear teeth.

The results obtained here are valid for the calculation of the bending strength at the base of the tooth of a gear with an involute profile, with a pressure angle of $20^{\circ}$ and tool correction limited to a head thickness of $e_{c} \leq 0.2 \mathrm{~m}$, where $m$ is the gear module, and with an equivalent number of teeth greater than 17 .

The paper is organized as follows: In Section 2, we explain the background to the study and in Section 3 we develop the analytical model calculation and the FEM technical implementation to the gear study. Also

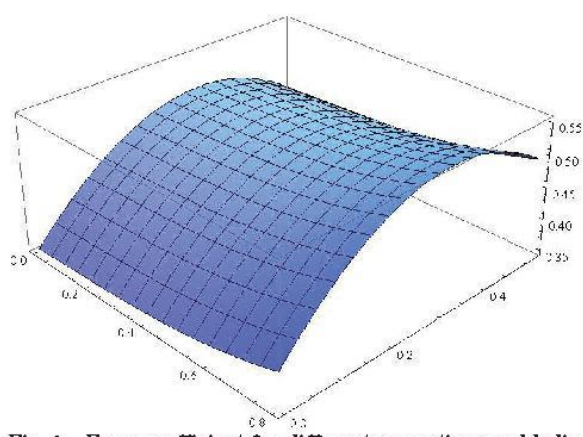

Fig. 1 Form coefficient for different corrections and helix angles $(\psi)$. included in Section 3 is the development for calculating $J$ factor. In Section 4 , we validate the model by comparing the results to the AGMA standard and in Section 5, we discuss the results and offer conclusions.

\section{Background}

It may be considered that the real importance of gears as mechanisms for the transmission of power began at the end of the nineteenth century, in parallel with the Industrial Revolution.

The first equation for calculating the bending strength force in the teeth of a gear was reported by Wilfred Luis, based on the calculation of a cantilever (with the particularities imposed by the complexity of the variable section of the tooth).

Considering the tooth of a toothed wheel as a cantilever beam with a critical section at the base of the tooth, Lewis [7] proposed the following as the value of its bending strength $(\sigma)$ :

$$
\sigma=\frac{W_{t}}{F \cdot m \cdot Y}
$$

where $W_{\digamma}$-tangential force on the tooth, $m$ - gear Module, $F$-width of tooth $\left(F=\lambda^{*} m\right)$, where $\lambda$ is a constant between 6 and 12, $Y$-the form factor, which takes into account the peculiarities of the tooth with respect to a constant-section beam.

Later it was observed that when a pair of gears moves at a moderate or high speed noise is generated due to the dynamic forces generated. The first evaluation of these efforts was described by Carl G. Barth at the end of the 19th century and was incorporated into Lewis' formula. This was finally left in the following form:

$$
\sigma=\frac{K_{v} \cdot W_{t}}{F \cdot m \cdot Y}
$$

where $K_{v}$ is speed factor.

As well as dynamic forces, the AGMA [8] takes into account the imprecisions in the generation of the tooth, the vibrations, the speed in the line of action and the misalignment of the shafts in order to be able to control such effects in the design. To determine the value of $K_{v}$, AGMA proposes the following equation: 
Determination of the Bending Strength Geometry Factor $\mathrm{J}$ for the Calculation of Stress in Parallel-Axis Gears as a Function of the Reference Correction and Slope Angle of the Gear Tooth

$$
K_{v}=\left(\frac{A+\sqrt{200 v}}{A}\right)^{B}
$$

where $v$ is velocity in the line of action in $\mathrm{m} / \mathrm{sg}$.

Also,

$$
\begin{aligned}
& A=50+56(1-B) \\
& B=0.25\left(12-Q_{v}\right)^{2 / 3}
\end{aligned}
$$

where $Q_{v}$ is a factor defining the quality of the gear with numbers from 8 to 12 , which indicate, from lesser to greater, the quality and exactness of the transmission of the gear.

The AGMA assesses the bending strength at the base of the tooth of a gear following the same Lewis principle; that is, adding to the original formula a series of factors $K$ (modifying values) in order to take into account different design and functioning conditions. It proposes the following as the value of the shear strength:

$$
\sigma=\frac{W_{t}}{F \cdot m \cdot J} \frac{K_{\alpha \cdot K_{m}}}{K_{v}} K_{s} K_{B} K_{I}
$$

where Barth's $K_{v}$ values have been replaced by $K_{v}$ values given by the AGMA (a function $Q_{v}$ and the velocity in the line of action) and the value $Y$ given by Lewis has been replaced by the value $J$, called the AGMA form coefficient, with $K_{a}$-load factor, which takes into account the variations in the load on the tooth, $K_{m}$-non-uniform distribution factor of the load on the surface of the tooth, $K_{s}$-size factor, $K_{B}-$ a factor that takes into account the relationship between the thickness of the ring and the height of the tooth (recently introduced), $K_{I}-1.42$ for idler or intermediate gears and 1.0 for the others.

The above AGMA factors take into account the possible real conditions that occur during the functioning of a gear.

The bending strength geometry factor (or form coefficient, $J$ ) with tooth displacement and slope angle is determined by the AGMA [8] using a complicated algorithm, defined in norm 908-B89. This algorithm demands the acquisition of many, hampering its generalization.

\section{A New Method for Calculating the $\mathbf{J}$ Factor}

As mentioned at the beginning of this work, our aim is to determine the form factor $J$ for gears in SI units by means of a novel calculation method that generalizes the solution by incorporating into the formulation the displacement of the tool when milling the tooth of a gear, or summary correction $(X)$ and the helix angle of the tooth $(\psi)$.

For this calculation, in the case cylindrical gears with straight and helical teeth with summary correction, a simple formulation was achieved using simulations with gears modeled in 3D and subjected to pairs of loads similar to those that the tooth of a gear can support within the elastic limit of the material.

The path followed to obtain the value of $J$ involved the following protocol:

- Determination of the value of $J$ using the algorithm of the AGMA norm 908-B89 for parallel-axis gears and a helix angle $\psi$ between $0^{\circ}$ (spur gear) and $30^{\circ}$ with summary correction $X$ between 0 and 0.8 ;

- Calculation of the value of $J$ by means of assays with finite elements;

- Comparison of the results of the two methods;

- Elaboration of results graphics;

- Mathematical formulation of the results (value of J).

3.1 Calculation of the $J$ Factor according to AGMA for Spur Gears with Zero Correction

Currently, AGMA [9] obtains the J factor for spur and helical gears (for $X=0$ and $\psi=0$ ) using the following expression:

$$
J=\frac{Y}{K_{f} m_{N}}
$$

where $Y$-the value defined in AGMA 908-B89, not to be confused with the Lewis factor; $K_{f}$-force concentration factor at the base of the tooth of a gear; $m_{N}$-load sharing ratio. This is determined by the 
relationship between the width of the face of the tooth and the length of the line of contact or gear line.

Defining the latter,

$$
m_{N}=\frac{P_{N}}{0.95 Z}
$$

We have $P_{N}$, the pitch at the base of the tooth, and $Z$ the length of the line of action.

For values of $X$ and $\psi$ different from 0 , it is necessary to have a set of intermediate values in the calculation process that are only valid for each case in particular.

\subsection{FEM Simulations}

The 3D modeling of the teeth of the gears to be analyzed was performed using a program called "JAWSTRAIN", which belongs to the Department of Mechanical Engineering of the University School in Zamora (University of Salamanca), and runs on the Solid Edge platform.

For the simulation, the teeth of the pinion are connected with the gear teeth, subjecting the set to a torsion torque and observing the behavior of one of the teeth at the point of maximum force demand. The operation was repeated for different numbers of teeth, corrections and reference angles (Fig. 2). The results obtained allow the level of correspondence between the model developed and that proposed by the AGMA reference norm to be evaluated.
In sum, our aim was to gain further insight into the bending strength values at the base of the tooth under the effect of the force acting on the tooth due to the torque the tooth must transmit in order to make effective proposals in the evaluation of the work capacity of gears and establish a physical model that combines the precise estimation of the load capacity of the gear by means of a simple evaluation procedure.

A total of 180 evaluations of the Form Coefficient $J$ were carried out for 18-tooth gears with wheels of 75 , 60 and 33 teeth, with helix angles, $\psi, 5^{\circ}, 10^{\circ}, 20^{\circ}$ and $30^{\circ}$ and tool corrections, $X$, of $0,0.1,0.2,0.3,0.4,0.5$, $0.6,0.7$ and 0.8 .

It was observed that the values obtained with any of the modules were identical, such that the results were calculated for a module equal to 1 . These are shown in Tables 1-3.

The results obtained for the Form Coefficients J were represented on a three-dimensional plot (Fig. 1) as a function of the reference correction and the slope angle $(\psi)$.

\subsection{Mathematical Formulation of the Results}

Upon observing the plots obtained, and using the Mathematica program, we obtain the equation that allows the Form Coefficient $J$ to be related to the parameters: tool correction $X$; helix angle $\psi$, number of

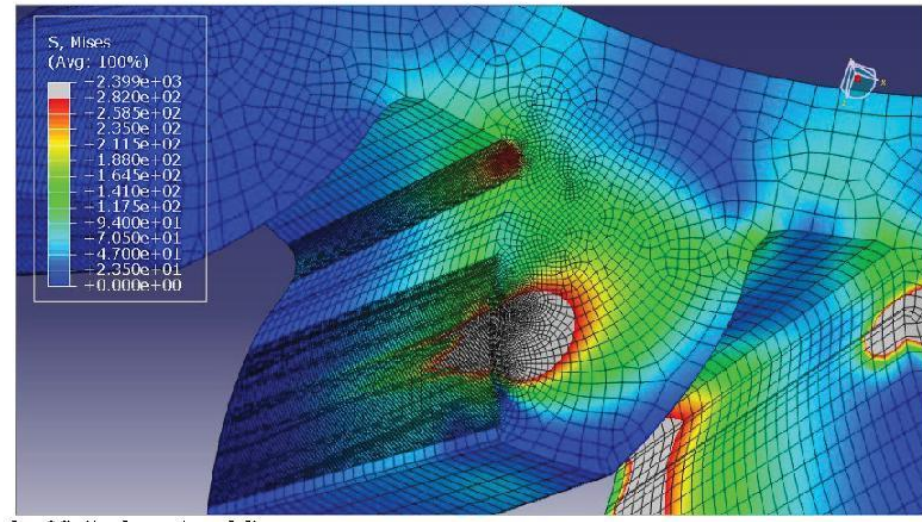

Fig. 2 Example of finite element modeling. 
Determination of the Bending Strength Geometry Factor $\mathrm{J}$ for the Calculation of Stress in Parallel-Axis 3 Gears as a Function of the Reference Correction and Slope Angle of the Gear Tooth

Table 1 FEM results (values of $J$ (for the form coefficient $N_{P}=18, N_{G}=75$ )) with differ ent corrections and helix angles.

\begin{tabular}{lllll}
\hline$X$ & $\psi$ & & & \\
\cline { 2 - 5 } & $5^{\circ}$ & $10^{\circ}$ & $20^{\circ}$ & $30^{\circ}$ \\
\hline 0.0 & 0.415857 & 0.4158570 & 0.48453398 & 0.50042504 \\
0.4 & 0.44749107 & 0.44749107 & 0.48992959 & 0.50921414 \\
0.8 & 0.46117559 & 0.46117559 & 0.51605752 & 0.55584254 \\
\hline
\end{tabular}

Table 2 FEM results (values of $J$ (for the form coefficient $N_{p}=18, N_{G}=60$ )) with differ ent corrections and helix angles.

\begin{tabular}{lllll}
\hline$X$ & $\psi$ & & & \\
\cline { 2 - 5 } & $5^{\circ}$ & $10^{\circ}$ & $20^{\circ}$ & $30^{\circ}$ \\
\hline 0.0 & 0.3959178 & 0.44526653 & 0.47436373 & 0.41104629 \\
0.4 & 0.40858823 & 0.45812613 & 0.48664885 & 0.42403786 \\
0.8 & 0.45033312 & 0.49891692 & 0.52781029 & 0.46481707 \\
\hline
\end{tabular}

Table 3 FEM results (values of $J$ (for the form coefficient $N_{F}=18, N_{G}=33$ )) with differ ent corrections and helix angles.

\begin{tabular}{lllll}
\hline$X$ & $\psi$ & & & \\
\cline { 2 - 5 } & $5^{\circ}$ & $10^{\circ}$ & $20^{\circ}$ & $30^{\circ}$ \\
\hline 0.0 & 0.37107256 & 0.42878506 & 0.44992027 & 0.40086193 \\
0.4 & 0.39067466 & 0.46966763 & 0.48839919 & 0.42419227 \\
0.8 & 0.433009211 & 0.59113390 & 0.52829704 & 0.45092891 \\
\hline
\end{tabular}

teeth on the pinion wheel $N_{P}$ and number of teeth of the gear with which $N_{G}$ meshes.

3.3.1 Incorporation of the Summary Correction in the Formulation of $J$

The displacement of the tool when milling the tooth of a gear " $X$ " varies the dimensions of this latter, the variation being most important at the base of the tooth modifying the bending force the tooth can support.

Here, this variation is reflected via the modification of the $J$ factor, multiplying this latter by an additional coefficient. One thus has

$$
J=J_{A G M A} \frac{S_{f}^{\prime}}{S_{f}}
$$

The negative corrections must be taken into account defining the term of the equation that multiplies $J_{A G M A}$ as

$$
\begin{gathered}
\left(\frac{S_{f t}}{S_{f}}\right) \text { if } X>1 \\
\left(\frac{S_{f t}}{S_{f}}\right)^{0} \text { if } X=1 \\
\left(\frac{S_{f t}}{S_{f}}\right)^{-1} \text { if } X<1
\end{gathered}
$$

where $J_{A G M A}$ is the form factor defined according to AGMA 908-B89 for spur gears $(\psi=0$ and $X=0) ; S_{f}$ is the thickness of the tooth with tool displacement and $S_{f}$ is the thickness of the tooth without tool displacement.

The thickness values at the base of the tooth are determined thus [10],

$$
\begin{gathered}
S_{f}=2 R_{f}(e v(\varphi)-e v(b)) \\
S_{f t}=2 R_{f}\left(e v\left(\varphi^{\prime}\right)-e v(b)\right)
\end{gathered}
$$

with $R_{f}$ the background radius of the gear without correction, $b$ - the angle measured at the base of the tooth, tangent to the directing circle, $\varphi$ - the angle measured at the tip of the tooth, the meeting of two curves that define the profiles of the teeth. $R_{f}^{\prime}, b^{\prime}$ and $\varphi^{\prime}$ correspond to the above parameters measured with the $X$ tool displacement

3.3.2 Incorporation of the Helix Angle, the Number of Teeth of the Pinion and of the Wheel in the Formulation of $J$

With the definition of the variation in $J$ due to the summary correction, it was possible to obtain the variation associated with the other variables, the equation that defines the Form Coefficient $\mathrm{F}$ having the following aspect: 
32 Determination of the Bending Strength Geometry Factor $\mathrm{J}$ for the Calculation of Stress in Parallel-Axis Gears as a Function of the Reference Correction and Slope Angle of the Gear Tooth

$$
J=J_{A G M A} \frac{s_{f}^{\prime}}{S_{f}}+\Delta J
$$

where $\Delta J$ is the increase in the factor due to the helix angle of $\psi$.

To determine the factor $\Delta J$ Napierian logarithms were applied to the equations obtained with the simulation data, obtaining straight lines converging on a point that fits the following equation:

where

$$
\begin{gathered}
\Delta J=\left[-2 \ln \left(m+n_{1}\right)\right] * \psi_{\text {Radians }}+ \\
{\left[\ln \left(m+n_{2}\right)\right] * \psi_{\text {Radians }}^{2}}
\end{gathered}
$$

$$
\begin{gathered}
m=\left(2.4^{*} 10^{-5} * N_{P}-2.5^{*} 10^{-3}\right) N_{G} \\
n_{1}=2.9 * 10^{-4} * N_{P}+0.63 \\
n_{2}=2.9 * 10^{-4} * N_{P}+0.2467
\end{gathered}
$$

and $N_{P}$ is the number of teeth of the pinion, $N_{G}$ is the number of teeth of the gear.

The values of $\Delta J$ calculated reach their maxima for angles (Fig. 3) of less than $20^{\circ}$, thereafter decreasing, and they become negative for very sharp helix angles.

From Eq. (14), and deriving $\Delta J$ with respect to $\beta_{a}$, the above maximum value is obtained.

$$
\psi=\frac{\operatorname{Ln}\left[m+n_{1}\right]}{\operatorname{Ln}\left[m+n_{2}\right]}
$$

\section{Checking the Results}

The results obtained upon using the proposed formula were compared with those obtained with the procedure specified by AGMA and that this latter summarizes in Ref. [11] for helical gears in cases different from those assayed here. The results are shown in Table 4 and prove to be concordant, with deviations of less than $4.5 \%$.

Up to now, the form factor J calculated corresponds to the tooth of the pinion of the gear because the former is the weakest part of the tandem and hence the one to be taken into account when making the design.

To check the validity of the proposed formulation, and since AGMA also gives values for it, we calculated the form factor $J$ for the tooth of the wheel of the same gear. The results are shown in Table 5 , with the observation of values similar to those obtained in the previous case (pinion). To perform this calculation in Eqs. (15)-(17), the values of $Z_{P}$ and $Z_{G}$ must be exchanged.

\section{Conclusions}

We have made a precise determination of the form factor for parallel-axis, straight and helical gears for any degree of correction incorporated into the formulation developed here.

We have compared the results with the international

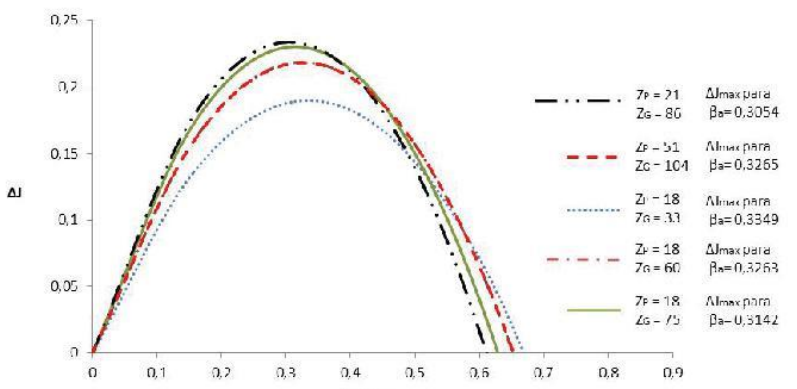

Fig. 3 Plot of $\Delta J$ as a function of $\psi$ for different cases $\left(Z_{P}^{B_{0}(r a d)} N_{F}, Z_{G}=N_{G}\right)$.

Table 4 Comparison of results for the form factor $J$ accor ding to AGMA 918-A93 and the proposed model.

\begin{tabular}{ll}
\hline Parallel-axis gear $\left(N_{P}=21 ; X_{P}=0.000 ; N_{G}=35 ; X_{G}=0.0000 ; \psi=30^{\circ}\right)$ \\
\hline According to AGMA 918-A93 & According to proposed model \\
\hline$J$ & $J$ \\
\hline 0.46 & 0.463 \\
\hline
\end{tabular}


Gears as a Function of the Reference Correction and Slope Angle of the Gear Tooth

Table 5 Comparison of results of for the form factor $J$ for the wheel of the gear according to AGMA 918-A93 and the prop osed model.

\begin{tabular}{ll}
\hline Parallel-axis gear $\left(N_{P}=21 ; X_{P}=0.000 ; N_{G}=35 ; X_{G}=0.0000 ; \psi=30^{\circ}\right)$ \\
\hline According to AGMA 918-A93 & According to proposed model \\
\hline$J$ (wheel) & $J$ (wheel) \\
\hline 0.48 & 0.483 \\
\hline
\end{tabular}

norms with a degree of correlation that has allowed us to validate the model.

A further result from this work is the ability to calculate the optimum helix angle for the design of parallel-axis gears.

Scope: The study was performed for gears with a number of teeth of 18 (or equivalent) up to 75 . The model proved to be valid for gears with up to at least 100 teeth.

\section{References}

[1] G.G. Rey, P.F. Fernández, R.J.G. Martin, Cylindrical gear conversions: AGMA to ISO, Gear Solution, 2006.

[2] G.G. Rey, P.F. Fernández, R.J.G. Martin, Finite elements method vs. ISO 6336 in the calculation of gear load capacity, in: Proceeding of 8th Pan-American Congress of Applied Mechanics 2004, pp. 169-172.

[3] G.G. Rey, P.F. Fernández, R.J.G. Martin, The finite elements method as altenative in gear design, Mechanical engineering Journal 5 (1) (2002) 55-67. (in Spanish)
[4] C. Ceballos, Gear model design using CAD systems, Faculty of Science Journal, University of Lima, Perú 1 (1) (2004) 23-27. (in Spanish)

[5] K. Varadi, R. Poller, Analys is of gear teeth contact by the finite element method, Acta Technica Acad. Sci. Hung 101 (5) (1988) 397-416

[6] R.G. Alvarez, Gear stresses determination using the finite element method, Thesis, Higher Polytechnic School of Zamora, University of Salamanca, 2004. (in Spanish)

[7] R.G. Budynas, J.K. Nisbett, Shigley's Mechanical engineering design, 9th ed., McGraw Hill, 2012, p. 710. (in Spanish)

[8] AGMA 2001-D04, Fundamental Rating Factors and Calculation Methods for Involute Spur and Helical Gear Teeth.

[9] AGMA 908-B89, Geometry Factors for Determining the Pitting Resistance and Bending Strength of Spur, Helical and Herringbone Gear Teeth.

[10] A.L. Martinez, Kinematics and dynamics of machines, Polytechnic University of Madrid, 2012. (in Spanish)

[11] AGMA 918-A93, A Summary of Numerical Examples Demonstrating the Procedures for Calculating Geometry Factors for Spur and Helical Gears. 
"Simplified Mechanical Model for a Stress-Ribbon Monitorized Footbridge. Analytical and Experimental Results"

M. Cacho-Pérez, N. Frechilla, Ivan M. Diaz, A. Lorenzana

6WCSCM: Sixth World Conference on Structural Control and Monitoring. Barcelona, Spain, 15-17 July 2014 


\title{
Simplified mechanical model for a stress-ribbon monitorized footbridge. Analytical and experimental results.
}

\author{
M. Cacho-Perez ${ }^{1}$, N. Frechilla ${ }^{2}$, Ivan. M. Diaz ${ }^{3}$ and A. Lorenzana ${ }^{4}$
}

Abstract

Focused on a stress-ribbon footbridge, the analytic response for the static and modal problem corresponding to a simple suspended steel plate is studied. The typical mechanical response under its own weight (catenary) is modified to take into account the elongation and thermal effects. The equilibrium equations describing the problem are ordinary differential equations that require a pair of coupled nonlinear equations to calculate the vroblem are ordinary differential equations that require a pair of coupled nonlinear equations to calculate the
value of is very important not only for the determination of the deformed equilibrium configuration but also for it influence on both the modal analysis and the dynamic response of the suspended cable. Most of the analytica effort is paid for the vertical response of the equivalent suspended cable, that is believed to match, once updated, with the vertical response of the steel-plated stress-ribbon footbridge under study ( $80 \mathrm{~m}$ single span Pedro Gomez Bosque footbridge in Valladolid, crossing the Pisuerga River).

The dynamic problem is formulated following the Irvine's scheme and the free undamped vibration modes are obtained. These modes depend on the static deformed shape, that depends on the mecharical properties of the cable, the location of its ends and also on the temperature changes. The resultant nonlinear equations are linearized taking the static deformed shape as reference. In this way, the small amplitude free vibrations are linearized taking the static deformed shape as reference. In this way, the small amplitude free

As the footbridge is continuously monitorized (acceleration in 18 points, apart from temperature and wind speed and direction), output-only modal parameter estimation has been carried out for different temperatures and similar operation conditions (pedestrian occupancy and wind). In this way, it has been possible to identify natural modes and to correlate them with the analytical ones in a rank of usual environmental temperatures $\left(+5^{\circ} \mathrm{C},+35^{\circ} \mathrm{C}\right)$.

\section{INTRODUCTION}

Cables are common structural members in civil engineering. Although in most of the cases cables are working together with other structural members (beams, plates, ...), in the application studied in this paper (stress ribbon footbridge) cables lead (together with the abutments) the structural strength and stability. The simple idea of installing ropes and wooden slabs for crossing a river has evolved in modem bridge designs like the ones of U. Finsterwalder in mid-20th century followed by J.Strasky, among others. Most of stress ribbon footbridges are built in reinforced concrete using poststressing techniques. With that tipology, the concrete deck contributes with mass and transversal stability but are the active tendons the responsible for the static and dynamic behaviour. Other building alternative is to place precast concrete slabs over the main cables or plates, once pretensioned properly. In the footbridge under study, the plate is $L=85 \mathrm{~m}$ long made of steel, being its cross-section $b=3,6 \mathrm{~m}$ width and $t=30 \mathrm{~mm}$ thick. The objective of this work is to present a proper mathematical model for such a structure and to check its applicability in predicting not only the static behaviour but also modal parameters. For that, special attention is focused in the vertical direction, although also transversal and torsional effects are presented but only considering that those effects are not coupled between them neither with the vertical ones[1], [2], [3], [4], [5].

*This work was supported University of Valladolid (Spain) and CARTIF

${ }^{1} \mathrm{M}$. Cacho-Perez Author is with Escuela de Ingenierias Industriales, University of Valladolid, Paseo de] Cauce 59, Spain cacholeit.uva.es

${ }^{2} \mathrm{~N}$. Frechilla Researcher is with Escuela de Ingenierias Industriales, University of Valladolid noel iaf rechillabyahoo. es ${ }^{2}$ N. Frechilla Researcher is with Escuela de Ingenierias Industriales, University of Valladolid noeliaf rechill
${ }^{3}$ Ivan. M. Diaz Researcher is with Universidad Politecnica de Madrid, Madrid, Spain ivan . munoz@upm.es

IJan. M. Diaz Researcher is with Universidad Politecnica de Madrid, Madrid, Spain ivan .munoz@upm.es
${ }^{4}$ A. Lorenzana Author is with Centro Tecnologico CARTIF, Parque Tecnologico de Boecillo, 205, Boecillo Valladolid, Spain ${ }^{4}$ A. Lorenzana Author
antior@ecartif.es 


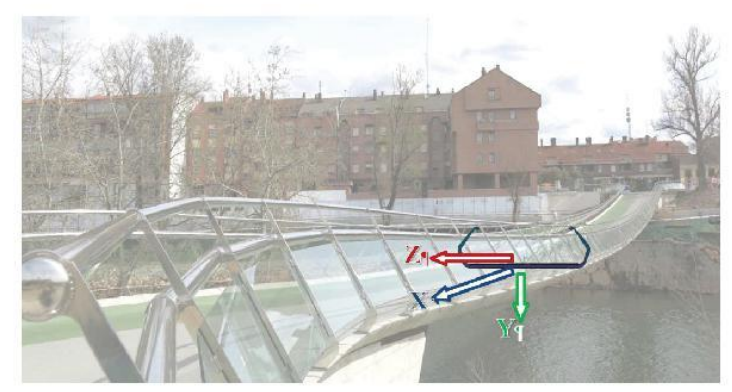

Fig. 1: Stress-ribbon footbridge under study

\section{STATIC ANALYSIS}

Being the plate placed in horizontal position (figure 1) where the width $b$ is along $Z$ axis, the static problem in the vertical plane $(X Y)$ can be approached using standard formulation for cables. Assuming material homogeneity and isotropy and considering only axial deformation normal to the cross-section, the only problem in solving the equilibrium equations of motion is to find the deformed shape configuration $C_{S}$ in which the equilibrium condition must be satisfied (figure 4). If $L_{0}$ is the stressless length of the cable and $C_{0}$ its original shape configuration for reference temperature $T_{0}$ (figure 2 ), the position of any cross-section $P(s)$ must be identified using the curvilinear coordinate $s$

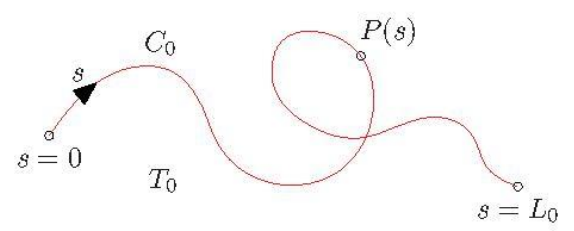

Fig. 2: Natural unstressed configuration $C_{0}$

With $N(s)$ being the axial force in the cable, figure 3 shows the left portion of the structure that must satisfied:

$$
\begin{aligned}
& N(s) \frac{d x}{d p}=H \\
& N(s) \frac{d y}{d p}=V-w s
\end{aligned}
$$

where $H$ and $V$ are the reactions in the left support $S_{A}$ and $w$ is the weight of the cable for unit stressless length. Geometrical relation between $x, y$ and $p(s)$ is given by

$$
\left(\frac{d x}{d p}\right)^{2}+\left(\frac{d y}{d p}\right)^{2}=1
$$




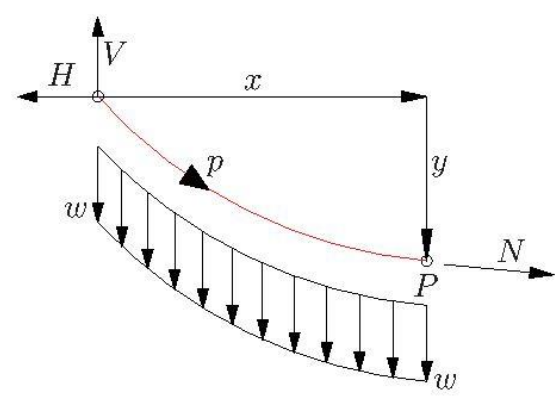

Fig. 3: Global static equilibrium

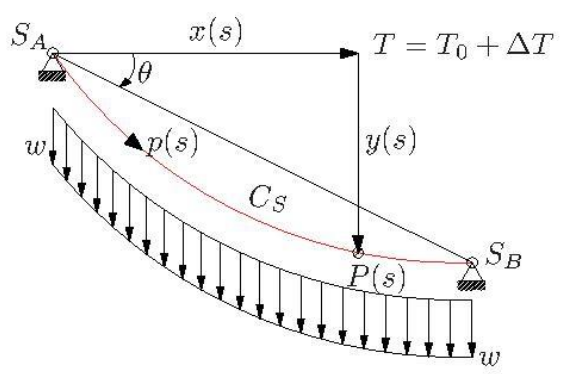

Fig. 4: Static equilibrium configuration $C_{s}$

and longitudinal static strain can be defined using

$$
\varepsilon(s)=\frac{d p}{d s}-1
$$

The former equations have to be completed with the following constitutive elastic law in terms of Young modulus $E$ and thermal expansion coefficient $\alpha$

$$
N(s)=E A(\varepsilon(s)-\alpha \Delta T)
$$

$A$ is the area of the cross-section of the cable and $\Delta T=T-T_{0}$ is the change in the temperature with respect to the reference one.

For solving the axial force $N(s)$ under vertical loading $w$, being its horizontal component constant $H$ and using (1) and (2), its modulus is

$$
N(s)=\left[H^{2}+(V-w s)^{2}\right]^{\frac{1}{2}}
$$

which depends on $s$. Introducing this expression on (2) and (4), the following differential equations are 
obtained

$$
\begin{aligned}
& \frac{d x}{d s}=\frac{H}{E A}+(1+\alpha \Delta T) \frac{H}{N(s)} \\
& \frac{d y}{d s}=\frac{(V-w s)}{E A}+(1+\alpha \Delta T) \frac{(V-w s)}{N(s)}
\end{aligned}
$$

that can be analytically solved with the following boundary conditions

$$
\begin{aligned}
& x(0)=0 \\
& y(0)=0 \\
& x\left(L_{0}\right)=L \\
& y\left(L_{0}\right)=L \tan \theta
\end{aligned}
$$

The solution is expressed through auxiliary functions $\Psi_{1}$ and $\Psi_{2}$ (see appendix A) in the following form:

$$
\begin{aligned}
& x(s)=\frac{H}{E A} s+\frac{H}{w}(1+\alpha \Delta T) \Psi_{1}(s) \\
& y(s)=\frac{V}{E A} s-\frac{1}{2} \frac{w}{E A} s^{2}+\frac{H}{w}(1+\alpha \Delta T) \Psi_{2}(s)
\end{aligned}
$$

Evaluating (8a,b) for $s=L_{0}$ leads to the following set of equations to be solved in $H$ and $V$ so the static problem is finally solved

$$
\begin{aligned}
& x\left(L_{0}\right)=\frac{H}{E A} L_{0}+\frac{H}{w}(1+\alpha \Delta T) \Psi_{1}\left(L_{0}\right)=L \\
& y\left(L_{0}\right)=\frac{V}{E A} L_{0}-\frac{1}{2} \frac{w}{E A} L_{0}^{2}+\frac{H}{w}(1+\alpha \Delta T) \Psi_{2}\left(L_{0}\right)=L \tan \theta
\end{aligned}
$$

Getting rid of the axial elongation and thermal effects, former equations become the classical hyperbolic functions known as catenary.

For the particular case of horizontally supported cables, $V=L_{0} w / 2$ and maximum deflection is obtained in the middle of the span. For any other case, in order to evaluate the position of the cross-section with maximum vertical displacement, the following equation has to be used, obtained through integration in (4)

$$
p(s)=(1+\alpha \Delta T) s+\frac{1}{2 w} \frac{H^{2}}{E A}\left[\Psi_{3}(s)+\Psi_{4}(s)\right]
$$

(see $\Psi_{3}$ and $\Psi_{4}$ in appendix A). With this expression is possible to extract the contribution of the thermal and elastic elongation. In the case of be interested in evaluating the displacement in the middle of the span, the following procedure should be used. First $s_{m}$ coordinate must be determined using:

$$
x\left(s_{m}\right)=\frac{H}{E A} s_{m}+\frac{H}{w}(1+\alpha \Delta T) \Psi_{1}\left(s_{m}\right)=\frac{L}{2}
$$

and then compute $z(s)=y(s)-x(s) \tan \theta$ resulting in the following equation

$$
\begin{gathered}
\delta=z\left(s_{m}\right)=\frac{V}{E A} s_{m}-\frac{1}{2} \frac{w}{E A} s_{m}^{2}+\frac{H}{w}(1+\alpha \Delta T) \Psi_{2}\left(s_{m}\right)-\frac{L}{2} \tan \theta \\
\text { III. MODAL ANALYSIS }
\end{gathered}
$$

Three different uncoupled problems can be derived for the mechanical approach to the stress ribbon footbridge under study. In first place, the Irvine scheme is followed to determinate vibration modes in the vertical direction in the deformed configuration. Then assuming probably not valid hypothesis the transversal vibrations could be addressed using the bending theory for beams in which the effect of axial force is taken into account. For the torsional vibrations, standard model for beams is attemped. 
III-A. Modes in vertical direction

According to classical theory for suspended cables, once static configuration $C_{s}$ is obtained, horizontal and vertical movements $u(s, t)$ and $v(s, t)$ can appear in dynamic loading (see fig. 5). Assuming that the amplitude of those movements is small with respect to the static deflection, dynamic configuration $C_{d}$ is always around static one $\left(C_{s}\right)$ and the equations of motion can be written down as

$$
\begin{aligned}
& \frac{\partial}{\partial p}\left[\left(N+N_{d}\right)\left(\frac{d x}{d p}+\frac{\partial u}{\partial p}\right)\right]=m \frac{\partial^{2} u}{\partial t^{2}} \\
& \frac{\partial}{\partial p}\left[\left(N+N_{d}\right)\left(\frac{d y}{d p}+\frac{\partial v}{\partial p}\right)\right]=m \frac{\partial^{2} v}{\partial t^{2}}-w_{d}
\end{aligned}
$$

where $w_{d}$ is the cable weight for unit length, $m$ is its mass for unit length under tension, $N$ is its axial force under static loading and $N_{d}$ is the variation in the axial force due to motion.

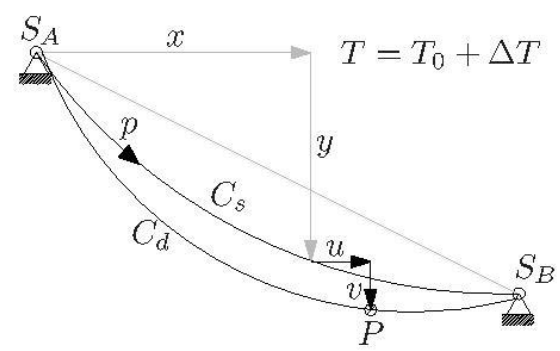

Fig. 5: Components of motion and dynamic configuration $C_{d}$

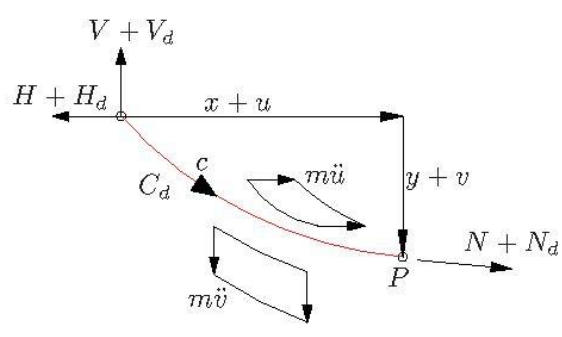

Fig. 6: Global dynamic equilibrium

III-B. Horizontal equilibrium

Introducing $H$ and $H_{d}$, defined as

$$
\begin{aligned}
& H=N \frac{d x}{d p} \\
& H_{d}=N_{d} \frac{d x}{d p}
\end{aligned}
$$


in (13a), the following equation can be reached, that define the dynamic behaviour of the cable in the horizontal direction

$$
\frac{\partial}{\partial x}\left[H \frac{\partial u}{\partial x}+H_{d}\left(1+\frac{\partial u}{\partial x}\right)\right] \frac{d x}{d p}=m \frac{\partial^{2} u}{\partial t^{2}}
$$

III-C. Vertical equilibrium

In a similar way, from (13b) the equation for the dynamic behaviour in the vertical direction became

$$
\frac{\partial}{\partial x}\left[H \frac{\partial v}{\partial p}+H_{d}\left(\frac{d y}{d p}+\frac{\partial v}{\partial p}\right)\right]\left(\frac{d x}{d p}\right)^{2}=m \frac{\partial^{2} v}{\partial t^{2}}
$$

Although (15) and (16) are valid for any value of the movements $u$ and $v$, it is necessary to introduce the hypothesis of small displacements in order to proceed with the modal analysis using the separation of variables method. First $H_{d}$ is considered constant along the cable, which means that dynamic horizontal elongation only change in time $(t)$, not in space $(p)$, that is

$$
\frac{\partial}{\partial x}\left(H_{d} \frac{d x}{d p}\right)=0
$$

so that at any cross-section $H_{d}$ depends only on the time according to

$$
H_{d} \frac{d x}{d p}=E A e(t)
$$

where $e(t)$ is the elongation function defined as

$$
e(t)=\varepsilon_{d}\left(\frac{d x}{d p}\right)^{2}
$$

where $\varepsilon_{d}$ is the longitudinal dynamic strain defined as

$$
\varepsilon_{d}=\frac{\partial c}{\partial p}-1
$$

that can be approximated using Lagrange approach in the following way

$$
\varepsilon_{d}=\frac{d x}{d p}\left[\frac{\partial u}{\partial x}+\frac{d y}{d x} \frac{\partial v}{\partial x}+\frac{1}{2}\left(\frac{\partial v}{\partial x}\right)^{2}\right]
$$

so that elongation function becomes

$$
e(t)=\frac{1}{L}\left[\int_{L} \frac{d y}{d x} \frac{\partial v}{\partial x} d x+\frac{1}{2} \int_{L}\left(\frac{\partial v}{\partial x} d x\right)\right] \approx \frac{1}{L} \int_{L} \frac{d y}{d x} \frac{\partial v}{\partial x} d x
$$

with these expresions (15) and (16) can be linearizated to

$$
\begin{gathered}
H \frac{\partial^{2} u}{\partial x^{2}}+E A e(t) \frac{\partial^{2} u}{\partial x^{2}}=m \frac{\partial^{2} u}{\partial t^{2}} \\
H \frac{\partial^{2} v}{\partial x^{2}}+E A e(t)\left(\frac{d^{2} y}{d x^{2}}+\frac{\partial^{2} v}{\partial x^{2}}\right)=m \frac{\partial^{2} v}{\partial t^{2}}
\end{gathered}
$$

Where $y(s)$ corresponding to the static configuration $C_{s}$. After integration using separation of variables technique, eigenvalues can be obtained using

$$
\omega_{L, i}^{2}=-\frac{(H+E A e(t)) \int_{0}^{L_{0}} \psi_{i}^{\prime \prime}(s) \psi_{i}(s) d s}{\rho A \int_{0}^{L_{0}} \psi_{i}^{2}(s) d s}
$$




$$
\omega_{V, i}^{2}=-\frac{(H+E A e(t)) \int_{0}^{L_{0}} \psi_{i}^{\prime \prime}(s) \psi_{i}(s) d s+E A e(t) \int_{0}^{L_{0}} y^{\prime \prime}(s) \psi_{i}(s) d s}{\rho A \int_{0}^{L_{0}} \psi_{i}^{2}(s) d s}
$$

where $\psi_{i}$ are the eigenvectors corresponding to pinned-pimned boundary conditions (note that the moment of inertica $I_{z}$ has been neglected).

III-D. Transversal modes

It is know that the equations of motion for axial and bending loading in a straight beam with constant cross-section of area $A$ and moment of inertia $I_{y}$ are

$$
\begin{aligned}
& V_{z}^{\prime}(s)+\rho A \omega_{n}^{2} u_{z}(s)=0 \\
& M_{z}^{\prime}(s)-H(s) \theta_{z}(s)+V_{z}(s)=0
\end{aligned}
$$

The eigenvalues can be obtained using

$$
\omega_{H, i}^{2}=\frac{E I_{y} \int_{0}^{L_{0}} \psi_{i}^{\prime \prime \prime \prime}(s) \psi_{i}(s) d s-\int_{0}^{L_{0}} H(s) \psi_{i}^{\prime \prime}(s) \psi_{i}(s) d s}{\rho A \int_{0}^{L_{0}} \psi_{i}^{2}(s) d s}
$$

where $\psi_{i}$ are the eigenvectors corresponding to clamped-clamped boundary conditions. Note that the former equation accounts for the effect of the axial force $H$ in the beam. Having in mind the low curvature of the stress ribbon footbridge, this bending theory could be applied for approximate its modal behaviour in the horizontal plane.

III-E. Torsional modes

Having in mind the geometry of the cross-section ( $30 \times 3600$ rectangle), the torsional behaviour can be approximated by Saint-Venant torsion theory, which is not coupled which bending neither with axial effects. The equation of motion is:

$$
G J M_{T}^{\prime}(s, t)=\rho I_{0} \ddot{\theta}_{x}(s, t)
$$

being $G J$ the torsional rigidity and $I_{0}$ the polar moment of inertia. In this case the eigenvectors are also the corresponding to clamped-clamped conditions and eigenvalues can be obtained using

$$
\omega_{T, i}^{2}=\frac{G J \int_{0}^{L_{0}} \psi_{i}^{\prime \prime}(s) \psi_{i}(s) d s}{\rho I_{0} \int_{0}^{L_{0}} \psi_{i}^{2}(s) d s}
$$

Again, an attempt to apply this torsional theory is done to approximate the torsional modal behaviour of the stress ribbon structure.

\section{APPLICATION TO THE STRESS-RIBBON FOOTBRIDGE}

The analytical model presented is applied to the Pedro Gomez Bosque footbridge, sited in Valladolid (Spain). This footbridge is a slender and lightweight steel stress-ribbon structure built in 2011 with only one span of $85 \mathrm{~m}$ that provides minimal impact on the surroundings. It mainly consists of a Corten steel sheet of $94 \mathrm{~m}$ long, $3,6 \mathrm{~m}$ width and only $30 \mathrm{~mm}$ thickness which is pre-tensioned and anchored to the two abutments, which are 2 meters not on a level. The complete steel sheet is fabricated by 8 -meter long plates welded. A number of 110 precast concrete slabs of $5,2 \mathrm{~m}$ long, $0,75 \mathrm{~m}$ width and $120 \mathrm{~mm}$ thickness lay on the steel sheet. The structure is completed by rubber pavement and a stainless steel and glass handrail. All these structural and functional parts suppose $23,6 \mathrm{KN} / \mathrm{m}$. Inital pre-tension on the steel sheet was adjusted so that displacement in the middle were limited to $\delta=L / 50$ with means $1,7 \mathrm{~m}$. Using the static model presented in section 2 the resulting axial tension is $H=12,536 \mathrm{MN}$ and the initial length should be 85,067 $\mathrm{m}$. Note that straight distance between abutments is $85,023 \mathrm{~m}$. 
IV-A. Analytical static results

Considering the service overload of $15,7 \mathrm{KN} / \mathrm{m}$ according to the structural project and for a rank of $\pm 15^{\circ} \mathrm{C}$ in temperatures with respect to the reference one $\left(T_{r e f}=20^{\circ} \mathrm{C}\right)$, the analytical values for displacements, axial force and deformed length are presented in table 1 for the relevant conditions considered.

IV-B. Analytical modal results

Table 2 shows natural frequencies for vertical and longitudinal vibrations according (23) and (24). Also, using (28) and (30), transversal and torsional frequencies are shown. Three different conditions are considered, depending on temperatures.

\begin{tabular}{rrrr}
$w(k N / m)$ & $\Delta T\left({ }^{\circ} \mathrm{C}\right)$ & $\delta(m)$ & $H(\mathrm{MN})$ \\
\hline 23,6 & 0,0 & 1,70 & 12,536 \\
23,6 & $-15,0$ & $\mathbf{1 , 5 4 7}$ & 13,792 \\
39,3 & $+15,0$ & 2,033 & 17,452 \\
39,3 & $-15,0$ & 1,773 & 20,035 \\
\hline
\end{tabular}

TABLE I: Static solution for different loading conditions

\begin{tabular}{|c|c|c|c|c|}
\hline$T_{\mathrm{ref}}=20^{\circ} \mathrm{C}$ & $\begin{array}{r}\text { longitudinal } \\
f_{L, i}(\mathrm{~Hz}) \\
\end{array}$ & $\begin{array}{r}\text { vertical } \\
f_{V, i}(\mathrm{~Hz}) \\
\end{array}$ & $\begin{array}{r}\text { transversal } \\
f_{H, i}(\mathrm{~Hz}) \\
\end{array}$ & $\begin{array}{l}\text { torsional } \\
f_{T, i}(\mathrm{~Hz})\end{array}$ \\
\hline $\begin{array}{l}\text { mode } 1 \\
\text { mode } 2 \\
\text { mode } 3 \\
\text { mode } 4\end{array}$ & $\begin{array}{l}0,75 \\
0,85 \\
1,67 \\
1,69\end{array}$ & $\begin{array}{l}0,93 \\
0,85 \\
1,30 \\
1,70\end{array}$ & $\begin{array}{r}2,76 \\
7,45 \\
14,47\end{array}$ & $\begin{array}{l}0,31 \\
0,63 \\
0,94 \\
1,26\end{array}$ \\
\hline \multicolumn{5}{|l|}{$\Delta T=+15^{\circ} \mathrm{C}$} \\
\hline $\begin{array}{l}\text { mode } 1 \\
\text { mode } 2 \\
\text { mode } 3 \\
\text { mode } 4\end{array}$ & $\begin{array}{l}0,76 \\
0,82 \\
1,66 \\
1,64\end{array}$ & $\begin{array}{l}0,97 \\
0,82 \\
1,27 \\
1,64\end{array}$ & $\begin{array}{r}2,75 \\
7,44 \\
14,46\end{array}$ & $\begin{array}{l}0,31 \\
0,63 \\
0,94 \\
1,26\end{array}$ \\
\hline \multicolumn{5}{|l|}{$\Delta T=-15^{\circ} \mathrm{C}$} \\
\hline $\begin{array}{l}\text { mode } 1 \\
\text { mode } 2 \\
\text { mode } 3 \\
\text { mode } 4\end{array}$ & $\begin{array}{l}0,75 \\
0,88 \\
1,68 \\
1,76\end{array}$ & $\begin{array}{l}0,89 \\
0,88 \\
1,34 \\
1,76\end{array}$ & $\begin{array}{r}2,77 \\
7,46 \\
14,48\end{array}$ & $\begin{array}{l}0,31 \\
0,63 \\
0,94 \\
1,26\end{array}$ \\
\hline
\end{tabular}

TABLE II: Analytical frequencies

IV-C. Experimental results

Some experimental values have been obtained through simple photogrammetry. According to photographs, the variation in the deflection in the central cross-sections for $35^{\circ} \mathrm{C}$ and $5^{\circ} \mathrm{C}$ is around $0,4 \mathrm{~m}$ which agrees with the analytical results shown in table 1 . Also some of the analytical modes has been observed for different temperatures by performing the corresponding operational modal analysis using the registered data obtained through monitoring. Table 4 presents the seven first natural frequencies identified by FDD and SSI techniques at $5^{\circ} \mathrm{C}, 20^{\circ} \mathrm{C}$ and $35^{\circ} \mathrm{C}$. Corresponding modes are shown in figure 7 . The notation used is as introduced in table III BZi for bending modes in the vertical XZ plane, BYi for bending modes in the horizontal XY plane and TXi for torsional modes around $\mathrm{X}$ axis. $\mathrm{i}$ the number of antinodes of the corresponding mode. No 


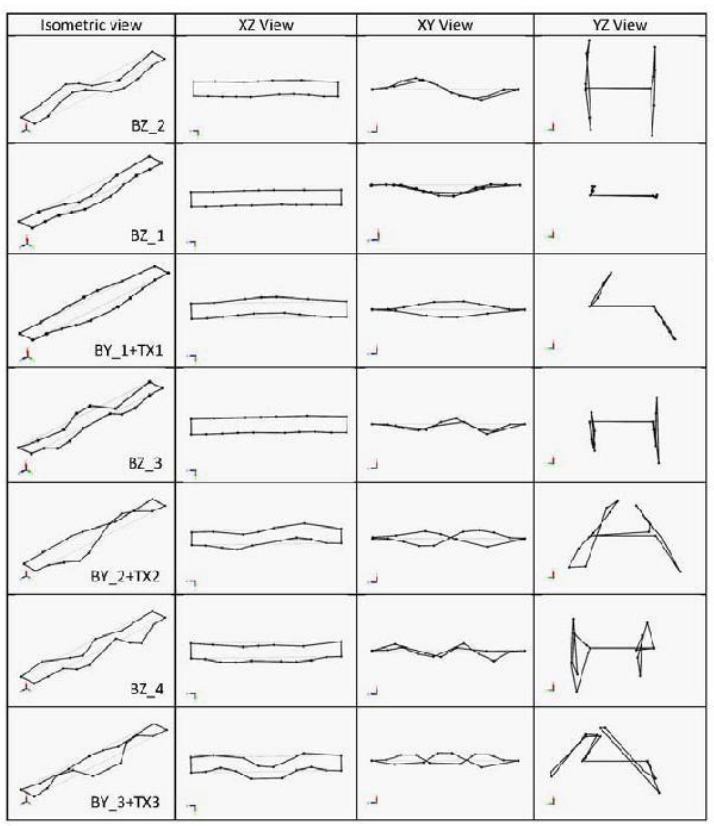

Fig. 7: Experimental mode shapes for the first seven modes

longitudinal modes have been observed and horizontal bending modes observed are always coupled with the torsional ones.

\section{CONCLUSions}

The evaluation of the static deformed shape of a suspended cables is a non-linear problem with analytical solution that depends not only on the location of its support, it weight and initial length but also on its elastic properties (cross-area and Young modulus) and on the temperature. Once the deflection at a reference central section is known, axial force can be deternmined, which is crucial for the computation of modal modes and frequencies. Stress ribbon footbridges behave like a suspended cable in the vertical plane. Static solution presented is according to the project estimations and agree with experimental values obtained using photogrammetry. With respect to the modal properties, also in the vertical plane it is possible to address the model and frequencies using analytical formulation. In this case, for vertical vibration modes, the numerical modal shapes and frequencies obtained reasonably agree to the experimental ones. The results are very sensitive on the axial force, that also depends on the temperature. In this way, once known the temperature, the determination of the axil force using the static solution is the starting point for the modal analysis. Changes about $15^{\circ} \mathrm{C}$ in the temperature with respect to the reference one can affect up to $5 \%$ in the modal frequencies. It is curious how that variation can be upward or downward and that the same tendency is observed in the experimental results. Table IV shows those tendencies for the first four modes in the vertical plane. Regarding 


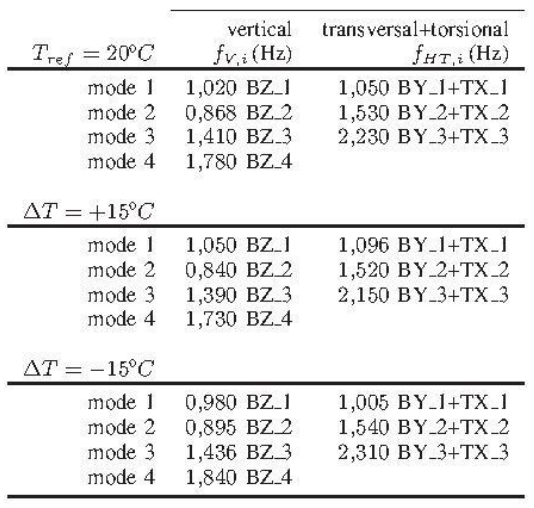

TABLE III: Experimental frequencies

\begin{tabular}{rrrrrrr} 
& \multicolumn{2}{c}{$5^{\circ} \mathrm{C}$} & \multicolumn{2}{c}{$20^{\circ} \mathrm{C}$} & \multicolumn{2}{c}{$35^{\circ} \mathrm{C}$} \\
moxperimental & Analytical & Experimental & Analytical & Experimental & Analytical & Experimental \\
\hline 1 & 0,980 & 0,89 & 1,020 & 0,93 & 1,050 & 0,97 \\
2 & 0,895 & 0,88 & 0,868 & 0,85 & 0,840 & 0,82 \\
3 & 1,436 & 1,34 & 1,410 & 1,30 & 1,390 & 1,27 \\
4 & 1,840 & 1,76 & 1,780 & 1,70 & 1,730 & 1,64 \\
& \multicolumn{7}{c}{ Mean value } & \multicolumn{2}{c}{ Variation for low temperature } & Variation for high temperature \\
\cline { 2 - 7 } mode & Analytical & Experimental & Analytical & Experimental & Analytical & Experimental \\
\hline 1 & 1,017 & 0,930 & $-3,9 \%$ & $-4,3 \%$ & $2,9 \%$ & $4,3 \%$ \\
2 & 0,868 & 0,850 & $3,1 \%$ & $3,5 \%$ & $-3,2 \%$ & $-3,5 \%$ \\
3 & 1,412 & 1,303 & $1,8 \%$ & $3,1 \%$ & $-1,4 \%$ & $-2,3 \%$ \\
4 & 1,783 & 1,700 & $3,4 \%$ & $3,5 \%$ & $-2,8 \%$ & $-3,5 \%$
\end{tabular}

TABLE IV: Comparison of frequencies of the vertical modes for different temperatures

transversal and torsional modes, although analytical formulation for beams predicts separated modes for both effects, experimental results clearly show perfect coupling between then that make the authors believe in the no applicability of the beam model for those effects, being necessary to resolve to plate or shell theory undergoing large displacements to get an initial approach for transversal and torsional vibrations in stress ribbon footbridges.

APPENDIX A. AUXILIARY FUnCTIONS

$$
\begin{aligned}
& \Psi_{1}(s)=\operatorname{arcsinh}\left[\tan \left(\varphi_{A}\right)\right]-\operatorname{arcsinh}[\tan (\varphi(s))] \\
& \Psi_{2}(s)=\left[1+\tan ^{2}\left(\varphi_{A}\right)\right]^{\frac{1}{2}}-\left[1+\tan ^{2}(\varphi(s))\right]^{\frac{1}{2}}
\end{aligned}
$$




$$
\begin{aligned}
& \Psi_{3}(s)=\tan \left(\varphi_{A}\right)\left[1+\tan ^{2}\left(\varphi_{A}\right)\right]^{\frac{1}{2}}-\tan (\varphi(s))\left[1+\tan ^{2}(\varphi(s))\right]^{\frac{1}{2}} \\
& \Psi_{4}(s)=\log \left[\tan \left(\varphi_{A}\right)+\left[1+\tan ^{2}\left(\varphi_{A}\right)\right]^{\frac{1}{2}}\right]-\log \left[\tan (\varphi(s))+\left[1+\tan ^{2}(\varphi(s))\right]^{\frac{1}{2}}\right]
\end{aligned}
$$

where

$$
\begin{aligned}
& \tan \left(\varphi_{A}\right)=\frac{V}{H}=\left.\frac{d y}{d x}\right|_{0} \\
& \tan (\varphi(s))=\frac{V-w s}{H}=\frac{d y}{d x} \\
& \text { VI. ACKNOWLEDGMENT }
\end{aligned}
$$

Authors wish to acknowledge to the partial support throught Research Project BIA201 1-28493-C02-02 ("Ministerio de Economía y Competitividad", Spanish Government).

\section{REFERENCES}

[1] J. Strasky, Stress ribbon and cable-supported pedestrian bridges. Thomas Telford Publishing, 2005.

[2] M. Lepidi and V. Gattulli, "Static and dynamic response of elastic suspended cables with thermal effects," Internationat Journat of Solids and Structures, vol. 49(9), pp. 1103-1116, 2012.

[3] A. Luongo and D. Zul]i, "Dynamic instability of inclined cables under combined wind flow and support motion," Nontinear Dynamics, vol. 67(1), pp. 71-87, 2012.

4] N. Bouaanani and M. lghouba, "A novel scheme for large deflection analysis of suspended cables made of linear or nonlinear elastic materials," Advances in Engineering Sofitware, vol. 42(12), pp. 1009-1019, 2011.

5] G. Sandovic, A. Juozapaitis, and R. Kliukas, "Simplified engineering method of suspension two-span pedestrian steel bridges with flexible and rigid cables under action of asymmetrical loads," Battic Journal of Road and Bridge Engineering, vol. 6(4), pp. 267-273, 2011. 



\section{BIBLIOGRAFÍA}


[1] Real Decreto 751/2011, de 27 de mayo, por el que se aprueba la Instrucción de Acero Estructural (EAE). Boletín Oficial del Estado. Madrid, 23 de junio de 2011, núm 149, pp 67148-67846.

[2] De Sebastián J. Análisis del Estado Límite de Servicio y Control de Vibraciones en pasarelas peatonales. Tesis Doctoral. Universidad de Valladolid. 2014.

[3] Real Decreto 1247/2008, de 18 de julio, por el que se aprueba la Instrucción de Hormigón Estructural (EHE-08). Boletín Oficial del Estado. Madrid, 22 de agosto de 2008, núm 203, pp 35176-35178 y suplemento.

[4] (2003). Recomendaciones para el proyecto de puentes metálicos para carreteras: RPM-95. $2^{\text {a }}$ reimpresión. Madrid. Centro de Publicaciones Secretaría General Técnica Ministerio de Fomento.

[5] (2003). Recomendaciones para el proyecto de puentes mixtos para carreteras: RPX95. $2^{\text {a }}$ reimpresión. Madrid: Centro de Publicaciones Secretaría General Técnica Ministerio de Fomento.

[6] Orden FOM/2842/2011, de 29 de septiembre, por la que se aprueba la Instrucción sobre las acciones a considerar en el proyecto de puentes de carretera (IAP 11). Boletín Oficial del Estado. Madrid, 21 de octubre de 2011, núm 254, pp 110164-110225.

[7] (2003). UNE-EN 1990:2003. Eurocódigos. Bases de cálculo de estructuras. Asociación Española de Normalización y Certificación AENOR.

[8] (2007). ISO 10137: 2007. Bases of for design of structures - Serviceability of buildings and walkways against vibrations. International Organization for Standardization.

[9] Charles P., Hoorpah W. (2006). Technical guide - footbridges - Assessment of vibrational behavior of footbridges under pedestrian loading. Paris: Service d'Etudes Techniques des Routes et Autoroutes (SETRA).

[10] (2005). FIB. Bulletin 32. Guidelines for the design of footbridges. Fédération internationale du béton (FIB). 
[11] Feldmann M., Heinemeyer C. y Lukic M. (2008). Design of Footbridges. Guideline. Human Induced Vibrations of Steel Structure (Hivoss). Hivoss-RFS2-CT-2007-00033.

[12] Hrenniskoff A. R. (1941) Solution of problemas in elasticity by the framework method. ASME J. Appl. Mech., 8, 169-175.

[13] McHenry D. (1943). A lattice analogy for the solution of plane stress problems. $J$. Inst. Civil Eng., 21, 59-82.

[14] Courant R. (1943). Variational methods for the solution of problems of equilibrium and vibrations. Bulletin of American Mathematical Society, 49, 1-43.

[15] Levy S. (1953). Structural Analysus and Influence Coefficients for Delta Wings. Journal of Aeronautical Sciences, 20(7), 449-454.

[16] Argyris J.H. y Kelsey S. (1960). Energy Theorems and Structural Analysis. London: Butterworths. (Collection of papers published in Aircraft Engineering in 1954 and 1955).

[17] Clough R. W. (1960). The Finite Element Method in Plane Stress Analysis. Proceedings of American Society of Civil Engineers. 2nd Conference on Electronic Computation. Pittsburgh, PA, 345-378.

[18] Archer J. S. (1965). Consistent Matrix Formulations for Structural Analysis Using Finite-Element Techniques. Journal of the American Institute of Aeronautics and Astronautics, 3(10), 1910-1918.

[19] Martin H. C. (1961) Plane Elasticity Problems and the Direct Stiffness Method. The Trend in Engineering, 13, 5-19.

[20] Gallagher R. H., Padlog J. y Bijlaard P. P. (1962). Stress Analysis of Heated Complex Shapes. Journal of the American Rocket Society, 32, 700-707.

[21] Melosh R. J. (1963). Structural Analysis of Solids. Journal of the Structural Division. Proceedings of the American Society of Civil Engineers, 205-223.

[22] Argyris J. H. (1964). Recent Advances in Matrix Methods of Structural Analysis. Progress in Aeronautical Science, 4, Pergamon Press, New York. 
[23] Clough R. W. y Rashid Y. (1965). Finite Element Analysis of Axisymmetric Solids. Journal of the Engineering Mechanics Division, Proceedings of the American Society of Civil Engineers, 91, 71-85.

[24] Wilson E. L. (1965). Structural Analysis of Axisymmetric Solids. Journal of the American Institute of Aeronautics and Astronautics, 3(12), 2269-2274.

[25] Turner M. J., Dill E. H., Martin H. C., y Melosh R. J. (1960). Large Deflections of Structures Subjected to Heating and External Loads. Journal of Aeronautical Sciences, 27(2), 97-107.

[26] Gallagher R. H. y Padlog J. (1963). Discrete Element Approach to Structural Stability Analysis. Journal of the American Institute of Aeronautics and Astronautics, 1(6), 1437-1439.

[27] Zienkiewicz O. C., Watson, M. y King, I. P. (1968). A Numerical Method of ViscoElastic Stress Analysis. International Journal of Mechanical Sciences, 10, 807-827.

[28] Zienkiewicz O. C. y Cheung, Y. K. (1965). Finite Elements in the Solution of Field Problems. The Engineer, 200, 507-510.

[29] Martin H. C. (1968). Finite Element Analysis of Fluid Flows. Proceedings of the Second Conference on Matrix Methods in Structural Mechanics, Wright-Patterson Air Force Base, Ohio, 517-535. (AFFDL-TR-68-150, Dec. 1969; AD-703-685, N.T.I.S.).

[30] Wilson E. L. y Nickel R. E. (1966). Application of the Finite Element Method to Heat Conduction Analysis. Nuclear Engineering and Design, 4, 276-286.

[31] Szabo B. A. y Lee G. C. (1969). Derivation of Stiffness Matrices for Problems in Plane Elasticity by Galerkin's Method. International Journal of Numerical Methods in Engineering, 1, 301-310.

[32] Zienkiewicz O. C. y Parekh C. J. (1970). Transient Field Problems: TwoDimensional and Three-Dimensional Analysis by Isoparametric Finite Elements. International Journal of Numerical Methods in Engineering, 2(1), 61-71.

[33] Lyness J. F., Owen D. R. J. y Zienkiewicz O. C. (1977). Three-Dimensional Magnetic Field Determination Using a Scalar Potential. A Finite Element Solution. 
Transactions on Magnetics, Institute of Electrical and Electronics Engineers, 13(5), 1649-1656.

[34] Zienkiewicz y Cheung (1967). The Finite Element Method in Structural and Continuum Mechanics. London: Mc Graw-Hill.

[35] Belytschko T. (1976). A Survey of Numerical Methods and Computer Programs for Dynamic Structural Analysis. Nuclear Engineering and Design, 37(1), 23-34.

[36] Belytschko T. (1976). Efficient Large-Scale Nonlinear Transient Analysis by Finite Elements. International Journal of NumericalMethods in Engineering, 10(3), 579-596.

[37] Caesar B. (1987). Updating system matrices using modal test data. Proc. Of the $5^{\text {th }}$ IMAC, 453-459, London.

[38] Ibrahim S.R. y Saafan A.A. (1987). Correlation of analysis and test in modelling of structures assessment and review. Proc. of the $5^{\text {th }} I M A C, 1651-1660$, London.

[39] Heylen W. y Sas P. (1987). Review of model optimisation techniques. Proc. of the $5^{\text {th }} I M A C, 1177-1182$, London.

[40] Natke H.G. (1988). Updating computational models in the frequency domain based on measured data: A Survey. Probabilistic Enngineering Mechanics, 4, 28-35.

[41] Imregun M. y Visser W.J. (1991). A review of model updating techniques. The Shock and Vibration Digest, 23(1), 9-20.

[42] Mottershead J.E. y Friswell M.I. (1993). Model updating in structural dynamics: A survey. Journal of Sounds and Vibration, 167(2), 347-375.

[43] Friswell M.I. y Mottershead J.E. (1995). Finite Element Model Updating in Structural Dynamics. Kluwer Academic publishers.

[44] Porras J.A., de Sebastian J., Casado C.M. et al. (2012). Modal mass estimation from output-only data using oscillator assembly. Mechanical Systems and Signal Processing, $26,15-23$.

[45] Sestieri A. y Ibrahim S. R. (1994). Analysis of errors and approximations in the use of modal coordinates. Journal of Sound and Vibration, 177(2), 145-157. 
[46] Ewins D. J. (2000). Modal Testing:theory, practice and applications. Research Studies Press Ltd.

[47] Alvin K. F. y Park K. C. (1994). Second-order structural identification procedure via state-space-based system identification. AIAA Journal, 32(2), 397-406.

[48] Ventura C.E., Lord J.F., Turek M., Brincker R., Andersen P. y Dascotte E. (2005). FEM updating of tall buildings using ambient vibration data. Proceedings of the sixth International Conference on Structural Dynamics (EURODYN), 4-7. Paris.

[49] Bernal D. y Gunes B. (2002). Damage localization in output-only systems: a flexibility based approach. Proceedings of the International Modal Analysis Conference (IMAC) XX, 1185-191. Los Angeles, California.

[50] Parloo E., Verboven P., Guillaume P. y Van Overmeire M. (2002). Sensitivity-based operational mode shape normalization. Mechanical Systems and Signal Processing, 16, $757-767$.

[51] Parloo E., Verboven P., Guillaume P. y Van Overmeire M. (2001). Proceedings of the International Conference on structural System Identification, 267-636.

[52] Brincker R. y Andersen P. (2003). A way of getting scaled mode shapes in output only modal analysis. Proceedings of the International Modal Analysis Conference (IMAC) XXI, paper 141. Orlando.

[53] López Aenlle M., Fernández P., Brincker R. y Fernández Canteli A. (2010). Scaling factor estimation using an optimized mass change strategy. Mechanical Systems and Signal Processing, 24, 3061-3074.

[54] Fernández P., López Aenlle M., Villa L. y Brincker R. (2007). Scaling factor estimation using an optimized mass change strategy. Part2: experimental results. Proceedings of the International Operational Modal Analysis Conference (IOMAC), 429-436. Copenhagen.

[55] Khatibi M. M., Ashory M. R. y Malekjafarian A. (2009). Scaling of mode shapes using mass-stiffness change method. Proceedings of the International Operational Modal Analysis Conference (IOMAC), 699-706. Copenhagen. 
[56] López-Aenlle M., Brincker R., Pelayo F. y Canteli A.F. (2012). On exact and approximated formulations for scaling-mode shapes in operational modal analysis by mass and stiffness change. Journal of Sound and Vibration, 331, 622-637.

[57] Ibrahim S. R. y Mikulcik E. C. (1977). A Method for the Direct Identification of Vibration Parameters from the free response. Shock and Vibration Bulletin, 47(4), 183198.

[58] Ibrahim S. R. (1977). Random Decrement Technique for Modal Identification of Structures. Journal of Spacecraft and Rockets, 14(11), 696-700.

[59] Vold H., Kundrat J., Rocklin G. T., y Russell R. (1982). A Multiple-Input Modal Estimation Algorithm for Mini Computers. SAE Transactions, 91(1), 815-821.

[60] Juang J. N. y Pappa R. S. (1985). An Eigensystem Realization Algorithm for Modal Parameter Identification and Model Reduction. Journal of Guidance, Control, and Dynamics, 8(5), 620-627.

[61] Juang J. N., Cooper J. E., y Wright J. R. (1988). An Eigensystem Realization Algorithm Using Data Correlations (ERA/DC) for Modal Parameter Identification. Control Theory and Advanced Technology, 4(1), 5-14.

[62] Juang J. N., Phan M., Horta L. G., y Longman R. W. (1993). Identification of Observer/Kalman Filter Markov Parameters: Theory and Experiments. Journal of Guidance, Control, and Dynamics, 16(2), 320-329.

[63] Lus H., Betti R., y Longman R. W. (1999). Identification of Linear Structural Systems Using Earthquake - Induced Vibration Data. Earthquake Engineering and Structural Dynamics, 28, 1449-1467.

[64] Yang C. D. y Yeh F. B. (1990). Identification, Reduction, and Refinement of Model Parameters by the Eigensystem Realization Algorithm. Journal of Guidance, Control, and Dynamics, 13(6), 1051-1059.

[65] Tseng D. H., Longman R. W. y Juang J. N. (1994). Identification of Gyroscopic and Nongyroscopic Second Order Mechnical Systems Including Repeated Problems. Advances in Astronautical Sciences, 87, 145-165. 
[66] Tseng D. H., Longman R. W. y Juang J. N. (1994). Identification of the Structure of the Damping Matrix in Second Order Mechanical Systems. Advances in Astronautical Sciences, 87, 166-190.

[67] De Angelis M., Lus H., Betti R. y Longman R.W. (2002). Extracting Physical Parameters of Mechanical Models From Identified State-Space Representations. Journal of Applied Mechanics, 69(5), 617-625.

[68] https://www.ansys.com

[69] Moaveni S. (1999). Finite Element Ananlysis. Theory and Application with ANSYS. New Jersey: PRENTICE HALL.

[70] http://nereida.deioc.ull.es/ pcgull/ihiu01/cdrom/matlab/contenido/node2.html

[71] Carmon Barranco C., "El programa Mathematica como herramienta en la resolución de problema y ejercicos de matemáticas". http://www.eduinnova.es/monografias09/ene2010/MATHEMATICA.pdf

[72] Rodriguez Súnico M. (2005) Análisis Modal Operacional: Teoría y Práctica. Proyecto Fin de Carrera. Universidad de Sevilla.

[73] (2001) Problemas de vibraciones en estructuras. Madrid: Colegio de Ingenieros de Caminos, Canales y Puertos.

[74] Meirovitch L. (1986). Elements of Vibration Analysis. McGraw-Hill.

[75] Paz M. (1992). Dinámica Estructural. Teoría y Cálculo. Barcelona: Ed. Reverté, S.A.

[76] Chopra A.K. (2007). Dynamics of Structures. Theory and Applications to Earthquake Engineering. Upper Saddle River, N.J.: Pearson/Prentice Hall.

[77] Documentación de vibraciones de la asignatura "Elementos de Máquinas y Vibraciones”. Departamento de Ingeniería Mecánica, Energética y de Materiales de la Universidad de Navarra. http://www.imac.unavarra.es/web_imac/pages/docencia/asignaturas/emyv_documentaci on.html 
[78] De Miguel Tejada A. Análisis Dinámico de Estructuras en el Dominio de la frecuencia. Trabajo de Investigación tutelado. Universidad Politécnica de Madrid. 2011.

[79] Deraemaeker A., Worden K. (2010). New Trends in Vibration Based Structural Health Monitiring. CISM Courses and Lectures, vol. 520. SpringerWienNewYork.

[80] (2009) ISO 2041:2009 Mechanical vibration, shock and condition monitoringVocabulary. International Organization for Standardization.

[81] Vélez W., Gómez D. y Thomson P. (2009). Ajuste de Modelos de Elementos Finitos. Dyna. 76(158), 177-189.

[82] Madenci E. y Guven I.(2006). The Finite Element Method and Applications in Engineering Using ANSYS. Springer Science+Business Media.

[83] Atienza Pascual R. (2004). Técnicas de actualización aplicadas a la detección de daños. Tesis. Universidad Politécnica de Madrid.

[84] Ziaei Rad S. (1997). Methods for Updating Numerical Models in Structural Dynamics. Tesis. University of London.

[85] Allemang R.J. (2003). The Modal Assurance Criterion - Twenty Years of Use and Abuse. Sounds and Vibration, 37(8), 14-21.

[86] Mares C., Friswell M.I. y Mottershead J.E. (2002). Model Updating Using Robust Estimation. Mechanical Systems and Signal Processing 16(1), 169-183.

[87] Horta L.G., Reaves M.C. y Voracek D.F. (2001). A Probabilistic Approach to Model Update. Proceedings of the First Annual Probabilistic Methods Conference. Newport Beach, CA.

[88] Martínez Calzón J., Moneo Vallés R. y De Teresa Trilla, E. (2007). Pasarela del Museo de la Ciencia sobre el río Pisuerga (Valladolid). Boletín Informativo Año $2007 n^{\circ} 8$ Construber, 10-13.

[89] Ladret P. y González A. (2005). Pretensado exterior en la pasarela del Museo de la Ciencia en Valladolid. Informes de la Construcción, 57(497). 
[90] Van Overschee P. y De Moor B. (1996). Subspace Identification for Linear Systems. Kluwer Academic Publishers.

[91] Kailath T. (1980). Linear Systems. Prentice Hall Inc.

[92] Melsa J. L. y Sage A. P. (1973). An Introduction to Probability and Stochastic Processes. Prentice-Hall Inc.

[93] Bendat J. S. y Piersol A. G. (1986). Random Data - Analysis and Measurement Procedures. John Wiley \& Sons.

[94] Lus H., De Angelis M., Betti R., y Longman R. W. (2003). Constructing secondorder models of mechanical systems from identified state space realizations. Part I: Theoretical discussions. Journal of Engineering Mechanics-ASCE, 129(5), 477-488.

[95] Reggio A., De Angelis M., y Betti R. (2013). A state-space methodology to identify modal and physical parameters of non-viscously damped systems. Mechanical Systems and Signal Processing, 41(1-2), 380-395.

[96] Allemang, R.J. (2000). Vibrations: Analytical and Experimental Modal Analysis, UC-SDRL-CN-20-263-662.http://www.sdrl.uc.edu/academic-course-nfo/docs/ucme662.

[97] Møller N., Gade S. y Herlufsen H. (2005). Stochastic Subspace Identification Technique in Operational Modal Analysis. International Operational Modal Analysis Conference. Copenhagen.

[98] E. Balmes (1997). New Results on the Identification of Normal Modes from Experimental Complex Modes. Mechanical Systems and Signal Processing, 11(2), 229243.

[99] Cacho-Pérez M., Frechilla N. y Lorenzana A. (2017). Estimación de las masas modales de una estructura en servicio mediante transformación en el espacio de estados. Revista Internacional de Métodos Numéricos para Cálculo y Diseño en Ingeniería, 33(1), 123-128. DOI: 10.1016/j.rimni.2016.02.002.

[100] Cacho-Pérez M., Frechilla N. y Lorenzana A. (2016). Estimación de parámetros modales de estructuras civiles a partir de la función de respuesta en frecuencia. Revista 
Internacional de Métodos Numéricos para Cálculo y Diseño en Ingeniería. DOI: 10.1016/j.rimni.2016.02.001. 